EFFICIENT REPRESENTATION OF THE HYDROGRAPHIC STRUCTURE

OF THE NORTH ATLANTIC OCEAN

AND ASPECTS OF THE CIRCULATION FROM OBJECTIVE METHÓDS 1989

by

Ichiro Fukumori

B.S., University of Tokyo

(1983)

SUBMITTED IN PARTIAL FULFILLMENT OF THE

REQUIREMENTS FOR THE DEGREE OF

DOCTOR OF PHILOSOPHY

at the

MASSACHUSETTS INSTITUTE OF TECHNOLOGY

and the

WOODS HOLE OCEANOGRAPHIC INSTITUTION

March, 1989

(C) Ichiro Fukumori 1989

The author hereby grants to MIT and WHOI permission to reproduce and distribute copies of this thesis document in whole or in part.

Signature of the author

Joint Program in Oceanography,

Massachusetts Institute of Technology -

Woods Hole Oceanographic Institution

Certified by

Accepted by

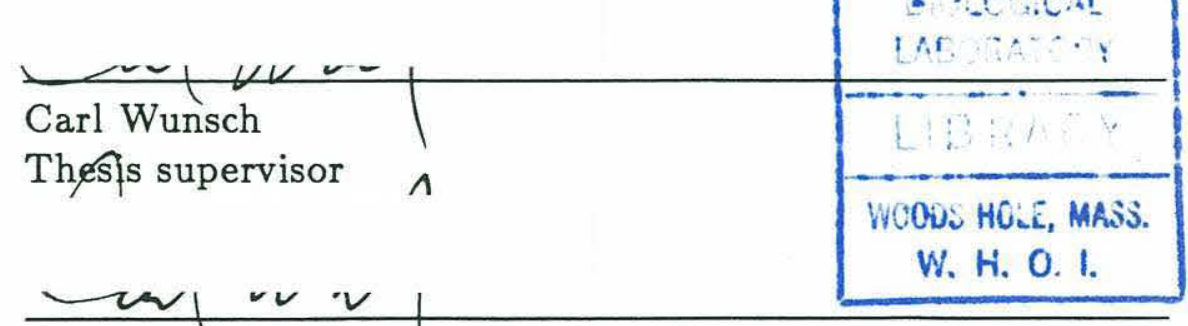

Chairman, Joint Committee for Physical Oceanography.

Massachusetts Institute of Technology -

Woods Hole Oceanographic Institution.

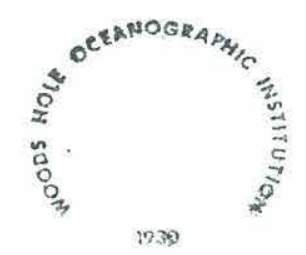


Efficient Representation of the Hydrographic Structure of the North Atlantic Ocean and Aspects of the Circulation from Objective Methods

by

Ichiro Fukumori

Submitted to the Massachusetts Institute of Technology - Woods Hole Oceanographic Institution Joint Program in March 1989 in partial fulfillment of the requirements for the degree of Doctor of Philosophy.

\begin{abstract}
The general theme of this thesis is the study of systematic mathematical techniques for determining the ocean circulation from classical hydrographic data. Two aspects of this theme are analyzed. The first is finding an efficient representation of hydrographic structure so as to make it most useful and informative. The second is application of inverse methods to the data to determine ocean circulation. Both subjects are examined in the North Atlantic Ocean.
\end{abstract}

The efficient representation is examined in terms of empirical orthogonal functions (EOFs) among the variations in vertical hydrographic profiles. The data used are of a new set of high quality hydrography, all obtained in the early 1980s. Common EOFs are examined among temperature, salinity, oxygen, phosphate, silicate, and nitrate. The EOFs identify a fundamental simplicity in the spatial distributions of these properties. Although the volume of numbers involved in the raw data is large, the significant degrees of freedom are only six in space and two among the six properties; temperature and salinity are represented by one mode, while the nutrients by another. The modal structure reflects some underlying simplicity in ocean physics. EOFs form a quantitative basis from which models of the ocean's hydrographic structure can be constructed for various degrees of complexities.

As for the second aspect, two applications of inverse methods are explored on small regional scales. The first problem addressed concerns the circulation inside a $12^{\circ}$ square located in the eastern basin over the axis of the Mediterranean Water tongue. The study is based on an ocean model constructed by mapping the modes identified in the first half of the thesis over the entire North Atlantic Ocean. A combination of box model inverse and $\beta$-spiral method is used to determine the geostrophic reference level velocities. The circulation consists of an anticyclonic circulation near the surface, which is part of the eastern half of the wind-driven subtropical gyre. The flow at depth is weak, and is a cyclonic circulation around the core of the Mediterranean Water tongue.

In the second inverse problem, we examine a decaying warm-core ring. Observations of a warm-core ring are used to formulate a model for diagnosing the physics of ring change over a two month period. About 30 hydrographic casts and acoustic 
doppler current measurements are used to generate estimates of an equivalent radially symmetric ring with radial contrasts of stratification, temperature, salinity, azimuthal velocity, angular momentum, and potential vorticity. A series of related models are inverted for the ring circulation and mixing coefficients. The circulation is insensitive to the model details, is well-resolved, and is a radial outflow and upwelling. Eddy coefficients are only partially resolved; determining the mixing with any degree of confidence appears to require a much more elaborate data set than the one available.

Thesis supervisor: Carl Wunsch

Cecil and Ida Green Professor of Physical Oceanography

Department of Earth, Atmospheric and Planetary Sciences

Massachusetts Institute of Technology 


\section{Table of Contents}

Abstract . . . . . . . . . . . . . . . . . 1

Introduction . . . . . . . . . . . . . . . . . . . 5

Chapter 1: Modal Analysis of a Hydrographic Section

1. Introduction . . . . . . . . . . . . . . . . . . . . . . . . . . 10

2. Hydrographic Structure along $64^{\circ} \mathrm{W}$. . . . . . . . . . . . . . 15

3. Preliminary Data Treatment . . . . . . . . . . . . . . . . . . 16

4. Vertical Modes along $64^{\circ} \mathrm{W}$

4.1 Empirical Orthogonal Functions . . . . . . . . . . . . . . . 19

4.2 Calculation of the Modes . . . . . . . . . . . . . . . . . 22

5. Determination of the Significant Degrees of Freedom . . . . . . . . 32

6. Discussion

6.1 Correlation between Different Properties . . . . . . . . . . 46

6.2 Dynamic Height Perturbations . . . . . . . . . . . . . . . . 54

$6.3 \mathrm{~T}-\mathrm{S}$ Relations etc. . . . . . . . . . . . . . . . . . . 57

7. Summary . . . . . . . . . . . . . . . . . . . . . . 63

Chapter 2: Hydrographic Modes of the North Atlantic Ocean

1. Introduction . . . . . . . . . . . . . . . . . . . . . . 65

2. Data Description . . . . . . . . . . . . . . . . . . . 67

3. Normalization

3.1 Range of Stations . . . . . . . . . . . . . . 86

3.2 Mean . . . . . . . . . . . . . . . . . . . . 88

3.3 Normalization Factor . . . . . . . . . . . . . . . 88

3.4 Areal Weighting . . . . . . . . . . . . . . . 9 90

4. Result of Modal Decomposition

4.1 Vertical Modes . . . . . . . . . . . . . . . . . . . . . . . 90

4.2 Singular Values . . . . . . . . . . . . . . . . . . 93

5. Residuals

5.1 Overall Residuals . . . . . . . . . . . . . . . . . 93

5.2 Spatial Variation of Residual . . . . . . . . . . . . . . . . . 97

5.3 Examples of Residual and Reconstruction Contours . . . . . . . 101

5.4 Summary . . . . . . . . . . . . . . . . . . . . 106

6. Resolution . . . . . . . . . . . . . . . . . . . . . . . . 106

7. Mass Flux

7.1 Comparison along Each Section . . . . . . . . . . . . . 111

7.2 Discussion . . . . . . . . . . . . . . . . . . . 121

8. Modal Coefficients

8.1 Mapping onto a Regular Grid . . . . . . . . . . . . . . . . 127

8.2 Mapped Expansion Coefficients . . . . . . . . . . . . . . . 132

8.3 Correlations among Different Property Expansion Coefficients . . 136

8.4 Modal Model . . . . . . . . . . . . . . . . . . . . . . 139

9. Summary . . . . . . . . . . . . . . . . . . . . . . . . . . . 139 
10. Conclusion . . . . . . . . . . . . . . . . . . . . 142

Chapter 3: Determining Circulation over the Mediterranean Tongue

1. Introduction . . . . . . . . . . . . . . . . . . . . . . . 146

2. General Hydrographic Structure . . . . . . . . . . . . . . . . . 149

3. Estimate of Absolute Velocity using $\beta$ Spiral Method

3.1 Review of Method . . . . . . . . . . . . . . . . 157

3.2 Formulation . . . . . . . . . . . . . . . . . . . 160

3.3 Result of $\beta$ Spiral . . . . . . . . . . . . . . . . . . . . 165

4. Box Model

4.1 Method and Formulation . . . . . . . . . . . . . 168

4.2 Is the $\beta$ Spiral Result Mass Conserving? . . . . . . . . . . . 172

5. Hybrid Model

5.1 Formulation . . . . . . . . . . . . . . . . 174

5.2 Result . . . . . . . . . . . . . . . . . . . . . . 176

6. Comparison to Other Observations . . . . . . . . . . . . . . . . 181

7. Summary and Conclusions . . . . . . . . . . . . . . . . . . . . 186

Chapter 4: Meridional Circulation and Mixing of a Warm-Core Ring Determined by Inverse Methods

1. Introduction . . . . . . . . . . . . . . . . . . . . . . . . . . 189

2. Structure of Ring $82 \mathrm{~B}$

2.1 Explanation of Data . . . . . . . . . . . . . . . . . . . . 192

2.2 Depth of Isopycnals . . . . . . . . . . . . . . . . . . . 195

2.3 Potential Temperature . . . . . . . . . . . . . . . . . 198

2.4 Azimuthal Velocity and Angular Momentum . . . . . . . . 200

2.5 Potential Vorticity . . . . . . . . . . . . . . . 205

2.6 Summary . . . . . . . . . . . . . . . 207

3. The Model . . . . . . . . . . . . . . . . . . . . . . . . . 207

4. Model 1: Diffusivity-Viscosity . . . . . . . . . . . . . . . . . 212

5. Other Models

5.1 Model 2: No Mixing . . . . . . . . . . . . . . . . . 217

5.2 Model 3: Non-Parameterized Mixing . . . . . . . . . . . . . 219

5.3 Model 4: Positive Mixing Coefficients . . . . . . . . . . . . . 221

5.4 Model 5: Thermocline Only . . . . . . . . . . . . . . . . 223

6. Summary . . . . . . . . . . . . . . . . . . . . . . . . . . . 225

Concluding Remarks . . . . . . . . . . . . . . . . . . . 227

Acknowledgments . . . . . . . . . . . . . . . . . . . . . . . 231

References . . . . . . . . . . . . . . . . . . . . 232 


\section{Introduction}

The volume of numbers traditionally required to describe the ocean is very large. A typical trans-ocean hydrographic section involves roughly 18,000 or more numbers, which might consist of 100 hydrographic stations, 30 depth levels distributed from sea surface to ocean floor, and 6 measured properties (temperature, salinity, oxygen, phosphate, silicate, and nitrate). In the case of a $\mathrm{CTD}-\mathrm{O}_{2}$ instrument, the vertical resolution is virtually continuous. Study of an entire ocean will involve several such trans-ocean sections, which will add another factor of 10 to the total amount of numbers. The World Ocean Circulation Experiment (WOCE) (WOCE International Planning Office, 1988) proposes to obtain global hydrographic measurements of unprecedented scope, involving more than 20,000 stations (7702 one-time global survey, 14,977 repeat hydrography). As a result, handling of these massive data sets will be tedious and the analyses complicated.

On the other hand, one might ask whether all the numbers are really necessary to describe the ocean. Examination of a plotted hydrographic section shows a relatively simple structure. Although there are minor undulations associated with transient eddies, the dominant features are the large scale coherences. Examples are the shape of the thermocline associated with the wind-driven circulation, and property contrasts that exist between different water masses (e.g. Mediterranean Water tongue), which structures extend over several stations and/or depth. What structure is significant is difficult to quantify and would differ depending upon ones' interest. We will consider the spatial variations associated with large percentages of the data variance as being the signal, and the unstructured residuals to be the noise. Much of this noise is undoubtedly due to mesoscale variability and measurement error. In this sense, there appears to be more degrees of freedom in a traditional description than may be warranted in describing such essential features of the ocean. 
If indeed the ocean structure can be described by limited degrees of freedom, we should be able to take advantage of this simplicity to efficiently analyze the underlying ocean circulation. The ocean circulation can be estimated from property distributions using a variety of objective methods; box model inverse methods (e.g. Wunsch and Grant, 1982), $\beta$ spiral methods (e.g. Olbers et al., 1985), and variational methods (e.g. Provost and Salmon, 1986). In general the methods involve setting up the data in a form to which constraints can be applied (e.g. smoothing in the $\beta$ spiral method), and simultaneously solving a large set of equations. Although these methods provide a straightforward procedure for estimating the circulation, the problem becomes impractically large for detailed basin-wide circulation studies, let alone analyses of the global ocean circulation. A quantitative identification of the underlying simplicity of ocean structure should provide insight into circulation analyses so as to allow their simplification.

In this thesis, we examine the essential degrees of freedom necessary to describe the ocean, and by doing so eliminate the redundancy present in the traditional form of hydrographic data. In essence, the problem is one of data reduction. A reduction procedure not only will be useful for analyzing large data sets, but could also suggest something about inherent physical mechanisms that cause the ocean to have the simple structure.

We examine the problem of identifying the significant degrees of freedom by analyzing the empirical orthogonal functions of the variations among vertical profiles of hydrographic data. Empirical orthogonal functions (EOFs) have been of popular use in identifying spatial patterns in temporal variations (e.g. Lorenz, 1956; Wallace and Dickinson, 1972; Davis, 1976; Wallace and Gutzler, 1981). Suk (1985) has used EOFs of density and temperature profiles to reduce the computational load in depth in a study of the tropical Atlantic circulation using variational methods. In this thesis, we examine both vertical and horizontal spatial patterns in hydrographic variations of 
several properties. The significance and implications of the modal structure underlying the North Atlantic Ocean's hydrography will be explored.

In a sense, traditional water mass analysis is also a reduction scheme. An entire section, or the whole ocean for that matter, can be described by a limited number of water masses, and from their distributions inferences are made on the underlying circulation (e.g. Wüst, 1935; Montgomery, 1938). Analyses on property-property diagrams are of common use to identify the water masses and to infer mixing that happens between the different types of water (e.g. Wright and Worthington, 1970). However, with the use of several types of tracers, these correlation diagrams become increasingly complicated (e.g. Broecker and Peng, 1982). With only 6 variables we can consider 15 different diagrams, and synthesizing all the correlations through different spaces, such as $\mathrm{T}-\mathrm{S}$ space, into an overall understanding of the ocean circulation becomes a confusing task. In addition, as each water mass is not strictly a point in property space, the descriptions of the water mass distributions are not exact, and the circulations based on these distributions remain qualitative. The new approach presented in this thesis is a quantitative description of the limited degrees of freedom, and the results can be thought of as an alternative to water mass description.

Yet another purpose of the data reduction is to identify independent data. That is, for some tracers the correlation with another is so tight that if we know one we can estimate the other with good accuracy (e.g. Worthington and Metcalf, 1961). In other words those two tracers do not provide independent information of water mass distribution. Thus by identifying such correlations, we may eliminate the need to measure some of the tracers, further minimizing the degrees of freedom and economizing the data acquisition at the same time.

The reduction procedure could also provide a basis for relating an instantaneous measurement to an average field. Analyses of basin-wide circulation using synoptic data often result in velocity structures that consist of cells of reversing flow on the 
order of $1000 \mathrm{~km}$ (e.g. Wunsch, 1978), which contrasts with the classic picture of the time-averaged smooth broad circulation pattern (e.g. Sverdrup et al., 1942). This alone merely indicates that at any given time the circulation is different from the average. In synoptic observations, there are transient eddies superposed on top of the time mean circulation. On the other hand, some of the results of these synoptic models appear to depend on the extent of smoothing of the data (Rintoul, 1988), so that estimating the mean by spatially smoothing synoptic data is a difficult problem. Property flux is a non-linear product derived from the data. Thus the flux associated with an averaged property distribution may not be consistent with an average of the individual fluxes. For example, a consistent circulation which conserves mass and heat over a finite region for the climatological atlas of Levitus (1982) may not exist (L.Memery personal communication 1988; Olbers et al., 1985). What we really lack is the understanding of the relations between an instantaneous field and an average field. More data may have to accumulate before enough knowledge is gained about the statistics, which would enable a proper reconstruction of the mean ocean. However, the identification and analysis of the minimum degrees of freedom that describe the major structures is a first step towards understanding these statistics of the ocean.

In the first chapter, we analyze a single north-south section in the subtropical North Atlantic to test the feasibility of data reduction. The issue of how the empirical orthogonal functions, or the modes, are calculated is discussed here in detail. We examine whether there are indeed limited degrees of freedom in the data (temperature, salinity, oxygen, phosphate, silicate, and nitrate) and whether such reduction retains the correct physics; e.g. mass flux, heat transport, and temperature-salinity correlation.

The region of analysis is expanded to the entire North Atlantic Ocean in Chapter 2. The hydrographic data are obtained between 1981 and 1983 (except for one section in 1985), and represent a modern high quality data set of the North Atlantic Ocean. 
The study's result may be viewed as a compact description of the climatological state of the North Atlantic Ocean in the early 1980's. Some of the physical significance of the modes will be examined.

The last two chapters examine some aspects of the ocean circulation using inverse methods. In Chapter 3, we examine the absolute circulation inside a $12^{\circ}$ square in the eastern North Atlantic Ocean, which straddles the Mediterranean Water tongue. The model is based on the modal analysis of Chapter 2. Whether the westward extending Mediterranean tongue is an advective feature or a result of diffusive processes is an interesting problem. In addition to the estimate of the inherent circulation in this region, this chapter provides a test to determine if the reduced data model of Chapter 2 is reasonable and useful for ocean circulation studies. The results will be compared with previous studies of the region.

Chapter 4 describes an application of the inverse method to a different phenomenon. In this chapter we estimate meridional (vertical and radial) circulation and mixing associated with a decaying warm-core ring, based on observed temporal changes in a particular ring's structure. Rings are observed to mix with their surrounding and these mixing processes are likely to be important in understanding the decay process at least in part. Mixing is an important process in ocean circulation. On the other hand, determination of mixing coefficients using synoptic hydrographic data is often difficult (e.g. Tziperman, 1987a). The analysis of Chapter 4 is a simpler situation where we follow a single water mass (i.e. the ring), so we might anticipate to do better.

Recently, inverse methods have received wide attention in oceanography, primarily within the context of estimating reference level velocities in geostrophic calculations, such as the study in Chapter 3. However, inverse problems are not limited to large-scale circulation problems. The analysis in Chapter 4 is a study to determine an ageostrophic flow and mixing. 


\section{CHAPTER 1 \\ Modal Analysis of a Hydrographic Section}

\section{Introduction}

Data used in traditional descriptions of the ocean's hydrographic structure appear to involve a much larger volume of numbers than might be necessary. Consider for example one north-south section in the North Atlantic Ocean (Figure 1-1). Here there are 92 stations and 35 depth levels (Section 3), which amount to more than 3,000 position numbers. At each position there are several different variables. With the 6 variables, namely, temperature, salinity, oxygen, phosphate, silicate, and nitrate, the total numbers approach 20,000 . However, to the eye, any one of the property contour plots (Figure 1-2) looks much simpler than what these numbers imply. Although there are short scale wiggles in the isolines, the dominant features are the long scale coherences, such as the bowl shape of the thermocline and the north-south gradient of the deep silicate, which extend over many stations and several depth levels.

In this and the next chapters we will examine this redundancy in a statistical framework. Specifically, what are the degrees of freedom necessary to describe the ocean? The objective of this study is to examine if indeed there are limited degrees of freedom, to identify them, and to study their physical significance. This part of the thesis is in essence an experiment on reduction of data. We will consider one procedure of analyzing spatial modes.

In this chapter, we analyze one north-south hydrographic section, Figure $1-1$, to examine the feasibility of modal analysis. The section is Endeavor cruise 129 made in April 1985 along a nominal longitude of $64^{\circ} \mathrm{W}$ (Knapp, 1988). The stations extend from just south of Nova Scotia into the Caribbean Sea. This particular hydrographic section is chosen, because it crosses the Gulf Stream, and has good horizontal resolution and nutrient data which we include in the analysis. A Gulf Stream crossing 


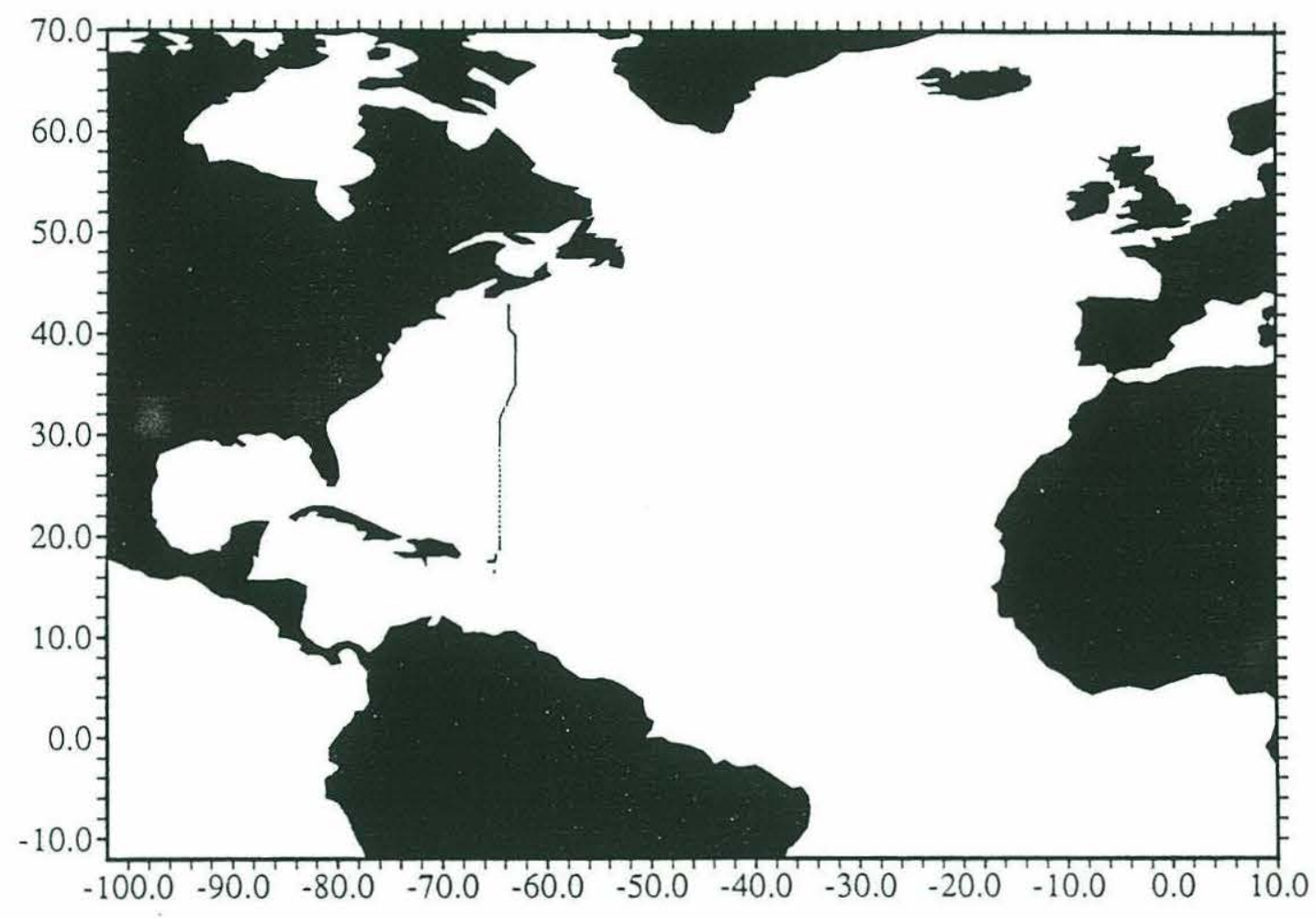

Figure 1-1. Station Locations of Endeavor Cruise 129. 

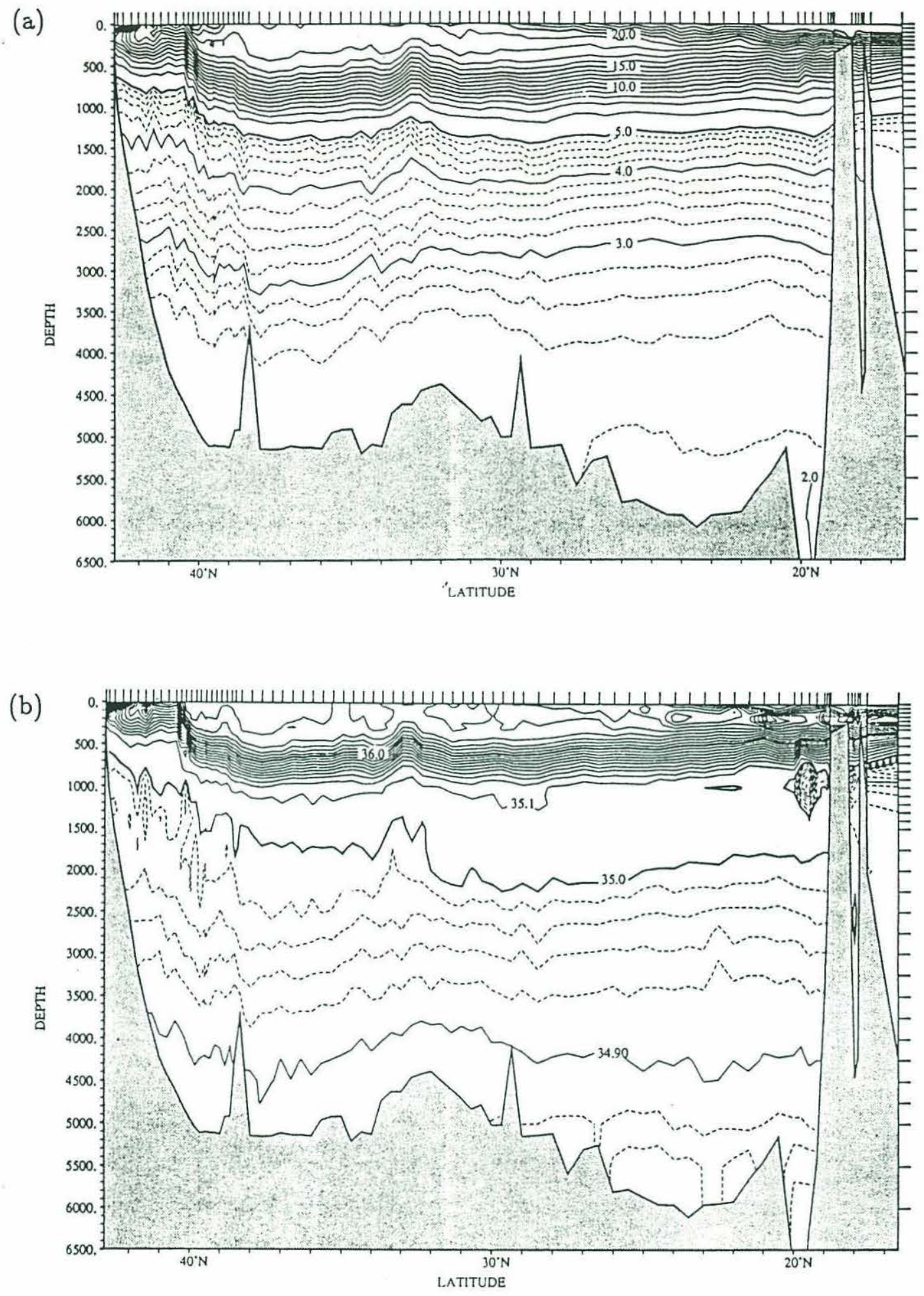

Figure 1-2. Property Distributions of Endeavor Cruise 129.

Tic marks at the top are station locations, while the ones on the right margin are the standard depths, discussed in Section 3. (a) Temperature $\left({ }^{\circ} \mathrm{C}\right)$ (b) Salinity (practical salinity units) 

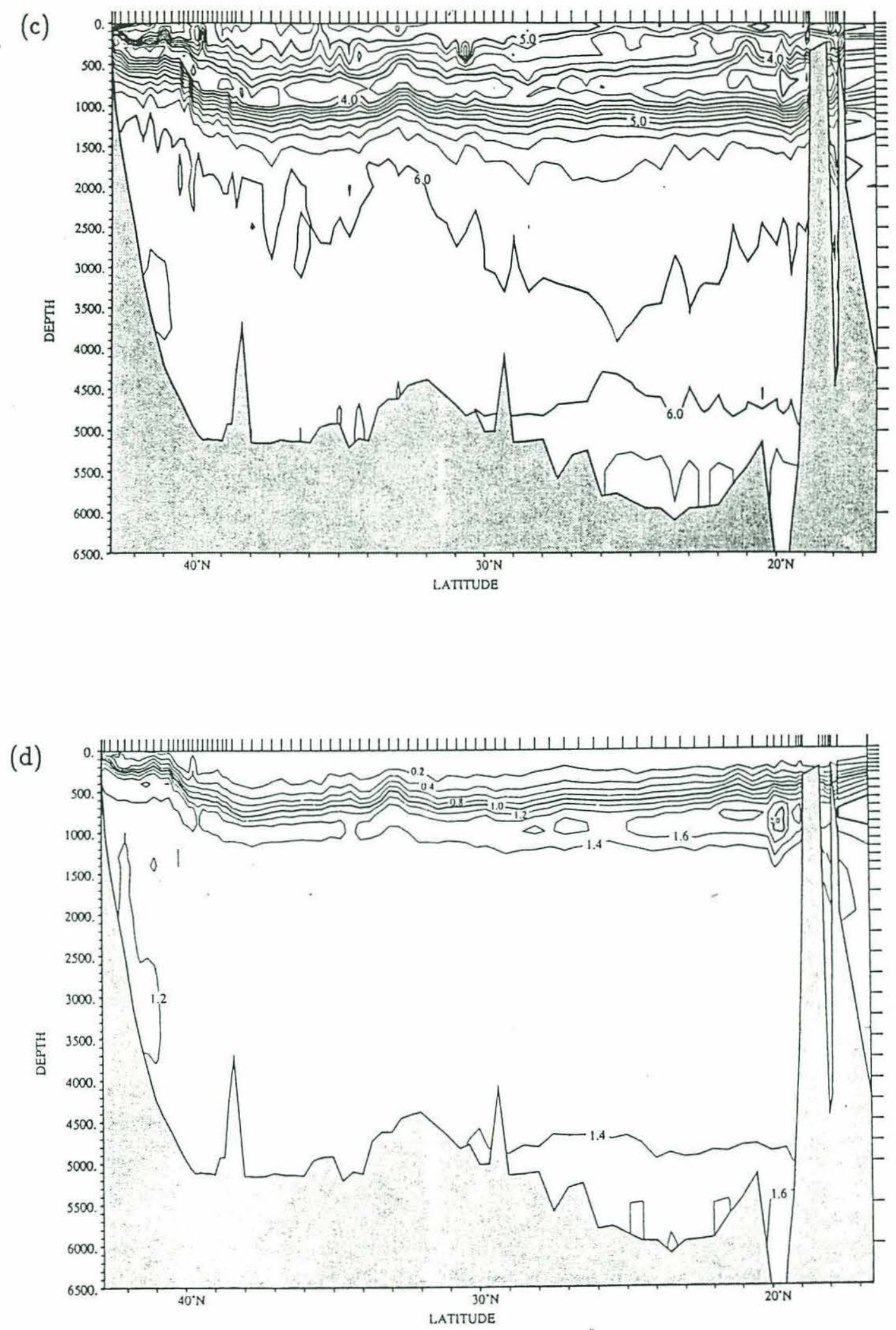

Figure 1-2. (continued) (c) Oxygen (ml/l) (d) Phosphate ( $\mu \mathrm{mol} / \mathrm{kg}$ ) 

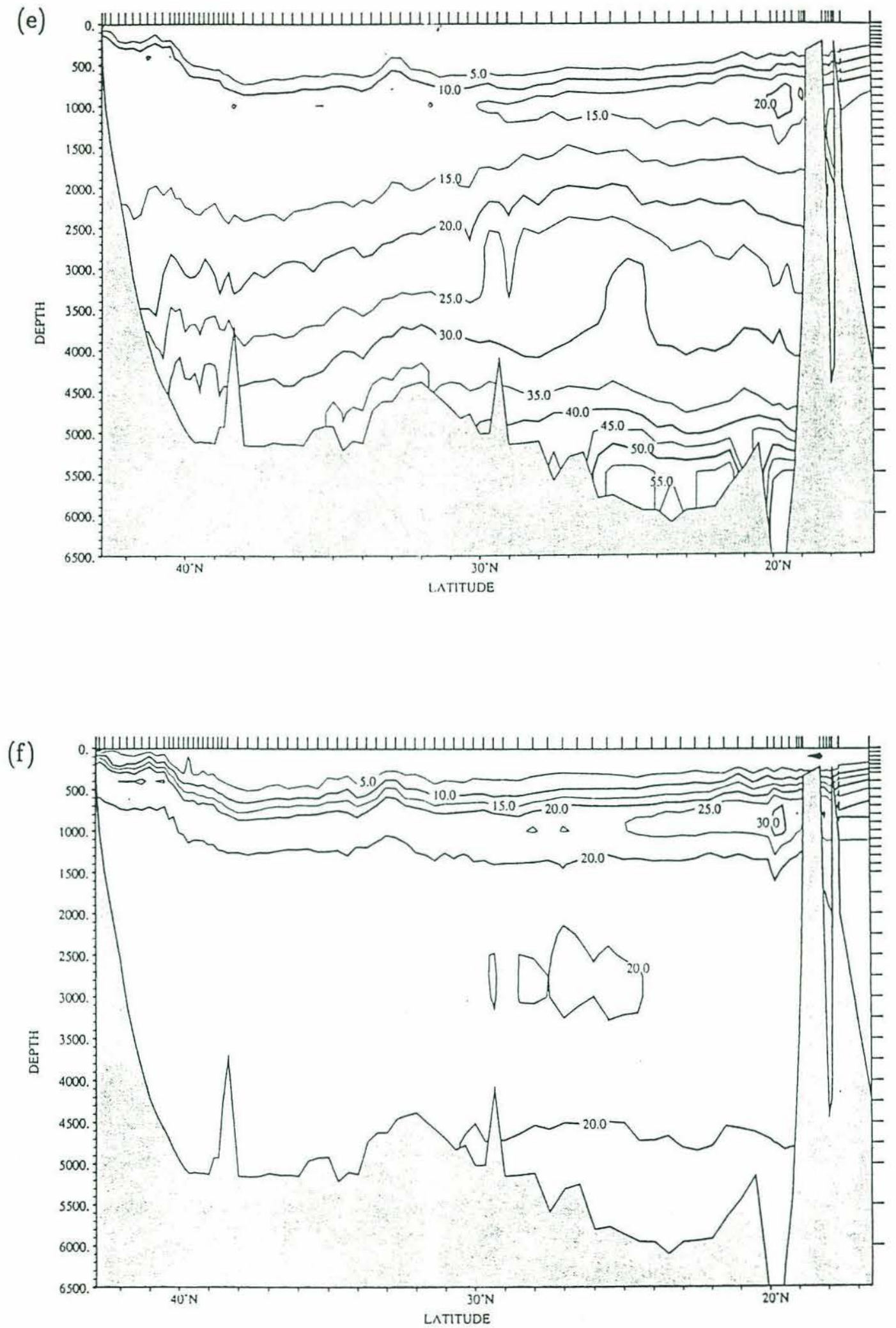

Figure 1-2. (continued) (e) Silicate $(\mu \mathrm{mol} / \mathrm{kg})$ (f) Nitrate $(\mu \mathrm{mol} / \mathrm{kg})$ 
was thought to be important to include in a first experiment, as it is associated with the strongest horizontal property gradient and is one of the major constituents of the general circulation that ought to be resolved.

We first review some of the hydrographic structure in Section 2. In Section 3, we describe the preliminary data treatment that is applied to interpolate the data onto a set of standard depths. All subsequent analyses take place at these depths. A detailed discussion of the derivation of the modes is presented in Section 4, while the analyses of what the minimum degrees of freedom is is given in Section 5. Additional examination of the modes' physical significance is discussed in Section 6. This chapter is ended with a summary and conclusion in Section 7 .

\section{Hydrographic Structure along $64^{\circ} \mathrm{W}$}

Figure 1-2 plots the contours of temperature, salinity, oxygen, phosphate, silicate and nitrate from the data interpolated to the standard depths described in the next section. Here some of the features of this hydrographic section, relevant to the discussions in the following sections, are described.

The section cuts through the subtropical gyre at its western end. The Gulf Stream is visible at about $40^{\circ} \mathrm{N}$ as sharply sloping isolines down towards the south in all 6 contour plots with a signature extending close to the bottom. There also appears to be a secondary steep gradient just south of the Gulf Stream at about $38^{\circ} \mathrm{N}$, perhaps due to a bifurcation of this current. The thermocline is deepest just south of this secondary dip and gradually rises to the south, resulting in the characteristic bowl shape of the anticyclonic subtropical gyre. The halocline exhibits a similar trend, and the isolines of oxygen just above the vertical oxygen minima $(\sim 800 \mathrm{~m})$ and nutrients above their local maxima $(\sim 1000 \mathrm{~m})$ all exhibit this bowl shape. There is a cold core ring at about $30^{\circ} \mathrm{N}$ that appears as an upward bump of the thermocline, whose effect is seen at least down to $2000 \mathrm{~m}$. 
Above the thermocline, the $18^{\circ} \mathrm{C}$ water appears as a nearly isothermal, isohaline water in the northern parts of the gyre. This water mass is capped by more warm and saline water further south, resulting in a typical wedge shape of the mode water.

Just below the thermocline towards the southern end at about $20^{\circ} \mathrm{N}$, we can see the effect of the Antarctic Intermediate Water which is characterized by being less saline, lower in oxygen and higher in nutrients than its surroundings. The Antarctic Bottom Water is also evident at the bottom at these southern latitudes, where we see the colder, fresher, less-oxygen but higher-nutrient abyssal water from $20^{\circ} \mathrm{N}$ to about $30^{\circ} \mathrm{N}$.

The northern edge of the Caribbean Sea is observed at the southern end of this section south of the ridge at $19^{\circ} \mathrm{N}$. Though the shallower depths $(\sim 1000 \mathrm{~m})$ are similar to the structures in the North Atlantic, deeper depths are different. Below $1500 \mathrm{~m}$, the Caribbean Sea is warmer, more saline, lower in oxygen with less nutrients than stations immediately to the north.

\section{Preliminary Data Treatment}

The original data were all interpolated vertically (in some instances horizontally as explained below) onto a set of depths on which later analyses are made. These depths are narrowly spaced in the upper waters, gradually widening toward the bottom. The spacings are chosen reasonably to resolve the large scale structures of the general circulation and most of the mesoscale activity. These depths are given in Table 1-1 and will be referred to as the standard depths. Here, we describe the steps taken to interpolate the data on to these depths.

There are two classes of data that are involved. One is the CTD $-\mathrm{O}_{2}$ data, where nearly continuous measurements of temperature, salinity and oxygen are made. These are first averaged into values every $2 \mathrm{dbar}$ from the surface to the maximum attained depth, which in most cases are within ten meters of the ocean floor. The other class 


\begin{tabular}{|c|c|c|}
\hline & depth & spacing \\
\hline 1 & 0. & \multirow{7}{*}{50.} \\
\hline 2 & 50. & \\
\hline 3 & 100. & \\
\hline 4 & 150. & \\
\hline 5 & 200. & \\
\hline 6 & 250. & \\
\hline 7 & 300. & \\
\hline 8 & 400. & \multirow{12}{*}{100.} \\
\hline 9 & 500. & \\
\hline 10 & 600. & \\
\hline 11 & 700. & \\
\hline 12 & 800. & \\
\hline 13 & 900. & \\
\hline 14 & 1000. & \\
\hline 15 & 1100. & \\
\hline 16 & 1200. & \\
\hline 17 & 1300. & \\
\hline 18 & 1400. & \\
\hline 19 & 1500. & \\
\hline 20 & 1750. & \multirow{14}{*}{250 . } \\
\hline 21 & 2000. & \\
\hline 22 & 2250 . & \\
\hline 23 & 2500 . & \\
\hline 24 & 2750 . & \\
\hline 25 & 3000. & \\
\hline 26 & 3250 . & \\
\hline 27 & 3500 . & \\
\hline 28 & 3750. & \\
\hline 29 & 4000. & \\
\hline 30 & 4250 . & \\
\hline 31 & 4500 . & \\
\hline 32 & 4750. & \\
\hline 33 & 5000. & \\
\hline 34 & 5500. & \multirow[t]{2}{*}{500.} \\
\hline 35 & 6000. & \\
\hline 36 & 7000. & \multirow[t]{2}{*}{1000.} \\
\hline 37 & 8000 . & \\
\hline
\end{tabular}

Table 1-1. The standard depths (dbar) 
is the water sample data which include the nutrients (phosphate, silicate and nitrate). These are obtained at discrete depths, usually at 24 or 36 different depths depending on the number of bottles on the water sampler.

Simple averages about the standard depths are obtained for the nearly continuous CTD $-\mathrm{O}_{2}$ measurements. This "window" was set $20 \mathrm{~m}$ above and $20 \mathrm{~m}$ below the standard depths. When the CTD does not reach the bottom to enable interpolation to all available standard depths, linear vertical extrapolation was allowed to estimate one more standard depth from the last two interpolated depths.

For the discrete bottle data, an Aitken-Lagrange interpolation was used (Roemmich, 1980). This cubic interpolation was constrained between values within the data used, such that when the interpolated values were larger than the maximum or smaller than the minimum of the raw data, the value at that standard depth was left unknown (flagged). It was also flagged when adjacent data spacing became too far apart. This window was set at $250 \mathrm{~m}$ between the surface to $400 \mathrm{~m}$, and $500 \mathrm{~m}$ between $400 \mathrm{~m}$ to $1200 \mathrm{~m}$. Any data shallower than $20 \mathrm{~m}$ was treated as though at the surface in the calculations. If necessary, linear vertical extrapolation is used to estimate one more depth beyond the last observed data, using the closest among the observed data and the Aitken-Lagrange interpolated standard depth estimate.

Some of the gaps left after this sequence are estimated by vertical linear interpolation and horizontal linear interpolation between adjacent stations within a section. Then these two interpolated values are plotted together against depth and are visually compared with the observed data. If either of these two appeared reasonable, the better value was accepted. Also as a check on the interpolation scheme of the bottle data at depth, the same visual examination was applied for all stations that have any consecutive bottles spaced two and a half times as large as or larger than the standard depth spacings. If any of the data were interpolated over too widely separated bottles with questionable results, they were flagged. Any gaps left after this were left flagged. 


\section{Vertical Modes along $64^{\circ} \mathrm{W}$}

\subsection{Empirical Orthogonal Functions}

The purpose of this section is to examine ways to quantify the redundancy present in the traditional form of hydrographic data so as to reduce the number of degrees of freedom. Consider just one of the section contours first, e.g. the temperature section in Figure 1-2a. The question may be put as: what is the dominant structural variation in this section? (The measure of this dominance or significance will be discussed in detail in Section 4.2. For the moment, let us assume that the data is nondimensionalized by some appropriate measure.) We can think of each station as a separate measurement and by comparing one with another, consider what the most common variation among them is, and then, analyze the second most common structure, and so on. From the procedure of its construction, we anticipate that we would only need a small number of these "common" structures or "modes" to reproduce the essential structures of the data. We can identify these "most common structures" as empirical orthogonal functions (EOF's) used in statistical studies of variation (e.g. Lorenz, 1956; Menke, 1984). Mathematically, they are the eigenvectors of the data covariance matrix, which in turn can be identified as the singular vectors of the data matrix. This relationship will be described below along with a geometric interpretation of the EOFs.

Write the data, the temperature section in Figure 1-2a, as a matrix, $A$, where each column represents a different station $(1$ through $m$ ) and each row a different standard depth ( 1 through $n$, where $n$ is 35 in the present section).

$$
A_{n \times m}=\left(\underline{t}_{1}, \underline{t}_{2}, \ldots, \underline{t}_{m}\right)
$$

( $A_{n \times m} ; n$-row, $m$-column matrix) $\underline{t}_{i}$ is a column vector representing the temperature profile at station $i$. In general, any rectangular matrix has a decomposition of form,

$$
A_{n \times m}=U \Lambda V^{T},
$$


where,

$$
\begin{aligned}
U_{n \times k} & =\left(\underline{u}_{1}, \underline{u}_{2}, \ldots, \underline{u}_{k}\right), \\
\Lambda_{k \times k} & =\left(\begin{array}{ccc}
\lambda_{1} & \\
& \ddots & \\
& & \lambda_{k}
\end{array}\right), \\
V_{m \times k} & =\left(\underline{v}_{1}, \underline{v}_{2}, \ldots, \underline{v}_{k}\right) .
\end{aligned}
$$

Here, $\lambda_{i}$ 's are ordered in decreasing value and $\underline{u}_{i}$ 's and $\underline{v}_{i}$ 's are orthonormal column vectors of dimension $n$ and $m$, respectively. $k$ is the rank of matrix $A$. This representation is called a singular value decomposition (SVD) (e.g. Lawson and Hanson, 1974). The $\underline{u}_{i}$ 's and $\underline{v}_{i}$ 's are termed singular vectors and the $\lambda_{i}$ 's the singular values.

The singular value decomposition has the following geometrical interpretation (e.g. Wiggins, 1972).

$\underline{u}_{1}$ is the most "common" column vector, in the sense that it has the largest projection for all of the columns in $A$ than any other unit vector.

$$
\left\|\underline{u}_{1}^{T} A\right\|=\operatorname{maximum}=\lambda_{1} .
$$

The norm $\|\cdot\|$ is defined as the square root of the sum of squares of all elements in the matrix;

$$
\|A\|=\sqrt{\sum_{i j} a_{i j}^{2}} .
$$

$\underline{u}_{2}$ is the second most common in the sense that, it has the largest projection after taking the projection from the first singular vector out.

$$
\left\|\underline{u}_{2}^{T}\left(A-\left(\underline{u}_{1} \underline{u}_{1}^{T} A\right)\right)\right\|=\text { maximum }=\lambda_{2} .
$$

The rest of the singular vectors follow in order. The same interpretation applies between the $\underline{v}_{i}$ 's and the rows of $A$.

From the above geometric argument, we can immediately identify the $\underline{u}_{i}$ 's as the vertical modes, and the corresponding row vectors, $\underline{v}_{i}$ 's, as the horizontal modes. In 
fact it turns out that the $\underline{u}_{i}$ 's and the $\underline{v}_{i}$ 's are the eigenvectors of the covariance matrix for the vertical and horizontal structures, respectively (which is the definition usually used for the EOFs). The covariance matrix of the vertical structure is $A A^{T}$,

$$
A A^{T}=\left(\underline{t}_{1} \underline{t}_{1}^{T}+\cdots+\underline{t}_{m} \underline{t}_{m}^{T}\right) .
$$

Then the $\underline{u}_{i}$ 's satisfy the following equations (Lawson and Hanson, 1974),

$$
A A^{T} \underline{u}_{i}=\lambda_{i} \underline{u}_{i}(i=1,2, \ldots k) .
$$

Similarly, $A^{T} A$ is the covariance matrix for the horizontal structures and the $\underline{v}_{i}$ 's satisfy,

$$
A^{T} A \underline{v}_{i}=\lambda_{i} \underline{v}_{i}(i=1,2, \ldots k) .
$$

The singular value, $\lambda_{i}$, can be interpreted as being a measure of the variance of $A$ accounted for by mode $i$. We can expand equation (1-1a) as,

$$
A=\lambda_{1} \underline{u}_{1} \underline{v}_{1}^{T}+\lambda_{2} \underline{u}_{2} \underline{v}_{2}^{T}+\cdots+\lambda_{k} \underline{u}_{k} \underline{v}_{k}^{T} .
$$

Each term represents contributions from a different mode. The variance that mode $i$ accounts for is calculated from the norm of its contribution to $A$ :

$$
\begin{aligned}
\left\|\lambda_{i} \underline{u}_{i} \underline{v}_{i}^{T}\right\|^{2} & =\lambda_{i}^{2}\left(\sum_{k l} u_{i k}^{2} v_{i l}^{2}\right)=\lambda_{i}^{2}\left(\sum_{k} u_{i k}^{2} \sum_{l} v_{i l}^{2}\right) \\
& =\lambda_{i}^{2}\left(\left\|\underline{u}_{i}\right\|=\left\|\underline{v}_{i}\right\|=1\right) .
\end{aligned}
$$

Thus the first mode accounts for the largest variance among all modes $\left(\lambda_{1}=\right.$ maximum), and the second mode for the second largest and so on. On the other hand, the total variance of $A$ is equal to the sum of all modal variance (Lawson and Hanson, 1974),

$$
\|A\|^{2}=\lambda_{1}^{2}+\lambda_{2}^{2}+\cdots+\lambda_{k}^{2} .
$$


Geometrically, equations (1-4b) and (1-4c) mean that the first mode contribution $\left(\lambda_{1} \underline{u}_{1} \underline{v}_{1}^{T}\right)$ is the closest approximation to $A$ such that,

$$
\left\|A-\underline{a} \underline{b}^{T}\right\|=\text { minimum, }
$$

for any two vectors $\underline{a}$ and $\underline{b}$.

The terms in the summation of equation (1-4a) are in decreasing order of their variance contribution. Thus a truncated summation of the RHS of equation (1-4a) represents an optimal approximation of $A$, in the sense that it is the closest representation for any given number of degrees of freedom (i.e. the number of modes). If the first few singular values are found to be dominant in magnitude, that would mean that a truncation by those few modes would almost reproduce $A$ entirely. This situation of a few dominant modes is what we would anticipate from the visual impression of Figure 1-2.

\subsection{Calculation of the Modes}

Here, we present the vertical modes of the Endeavor cruise 129 . We shall only deal with stations north of $21^{\circ} \mathrm{N}$, excluding the stations in the Caribbean Sea. Since many of the six variables (temperature, salinity, oxygen, phosphate, silicate, and nitrate) have features in common, we will consider modes based on all six variables at the same time, rather than calculating modes for each variable separately. For this purpose, we must normalize the data so that comparison between different variables, such as temperature and nitrate, could be made. For example, without nondimensionalization salinity has a magnitude of about 35 in practical salinity units while phosphate has about $1 \mu \mathrm{mol} / \mathrm{kg}$. Then the variance between these will be dominated by salinity, as it has the much larger magnitude. As in linear inverse problems (e.g. Wunsch, 1978), we must normalize (nondimensionalize) each variable to avoid this kind of artificial significance. On the

other hand, the dominant structure within each variable is its vertical stratification. 
The biggest change in any variable is the change over depth rather than over the width of the ocean. So it would make sense to remove this vertical mean profile out of the data and only consider the variation about it. Thus the normalization is;

$$
a_{i j}^{\prime}=\frac{a_{i j}-\bar{a}_{i}}{\sigma_{i}}
$$

where,

$a_{i j}$ is a variable at depth $\mathrm{i}$, station $\mathrm{j}$,

$\bar{a}_{i}$ is the mean at depth $i$,

$\sigma_{i}$ is the normalization factor at $\mathrm{i}$.

For the mean, $\bar{a}_{i}$, we will use the zonal mean at each standard depth for each variable. The normalization factor, $\sigma_{i}$, can be thought of as a measure of noise variance.

Here, we must define what we consider as signal and what as noise. The goal of the analysis is to retain and separate the large-scale time-averaged features from the time-dependent features and errors which, hypothetically, do not contribute to the overall property flux budget of the ocean (i.e. the ocean circulation). The percentage variance has commonly proved to be the most useful measure of significance and will be used here. We will regard, as noise, the unstructured residuals that are associated with small percentages of the data variance. Much of this noise is undoubtedly due to real mesoscale variability, and measurement error (including errors due to navigation, interpolation, instrument, etc) which is not an oceanographic signal. The variability associated with these noise errors is transient or random and is not likely to be resolved when combining separate hydrographic data sets. In this respect, we might regard this variability as not essential in describing the ocean. (We can not resolve it anyway.)

As we are concerned with the percentage, a relative measure of variance between different dimensional properties will suffice. We can always increase the number of modes retained until the appropriate variance is accounted for. With 35 modes (the number of standard depths used in this study), we always reproduce the data itself. The 
variance of the data about the mean contains the signal variance as well as the noise variance, and is not necessarily an appropriate measure for $\sigma_{i}$. The signal to noise ratio could be nonuniform with depth and between different variables, which makes the data variance inappropriate for the relative measure of noise. Assuming that the noise defined above has a short horizontal length scale, we could estimate it from the difference of each variable from neighboring stations. Conversely, we could say that this is the definition of the noise we refer to. Such a definition will include some of the Gulf Stream's short scale variance, but because we average the variations over the entire length of the section this will not dominate the estimated value. Also, as the Gulf Stream's signal appears in all variables that extend close to the bottom and as we want a relative measure, the presence of the Gulf Stream in the average should not cause a problem. (If it did, we could eliminate parts of the section that correspond to the Gulf Stream.)

Let $d_{i}$ be data at station $i$ at a certain depth, with signal $s_{i}$ and noise $n_{i}$ :

$$
d_{i}=s_{i}+n_{i}
$$

Then the root mean square (RMS) difference from a neighboring station $(i+1)$ is,

$$
\begin{aligned}
<\left(d_{i}-d_{i+1}\right)^{2}> & =<\left(s_{i}-s_{i+1}+n_{i}-n_{i+1}\right)^{2}> \\
& =<\left(s_{i}-s_{i+1}\right)^{2}>+2<n^{2}>,
\end{aligned}
$$

assuming the noise is uncorrelated over the distance and has uniform variance. If the signal has a much longer correlation distance than the station separation, we could neglect the first term in equation (1-8) with respect to the second term and have,

$$
<\left(d_{i}-d_{i+1}\right)^{2}>\approx 2<n^{2}>
$$

As the stations of this cruise are densely spaced more or less in accordance to the scales of the fluctuations (denser over the Gulf Stream and coastal areas, and wider in the 
A.

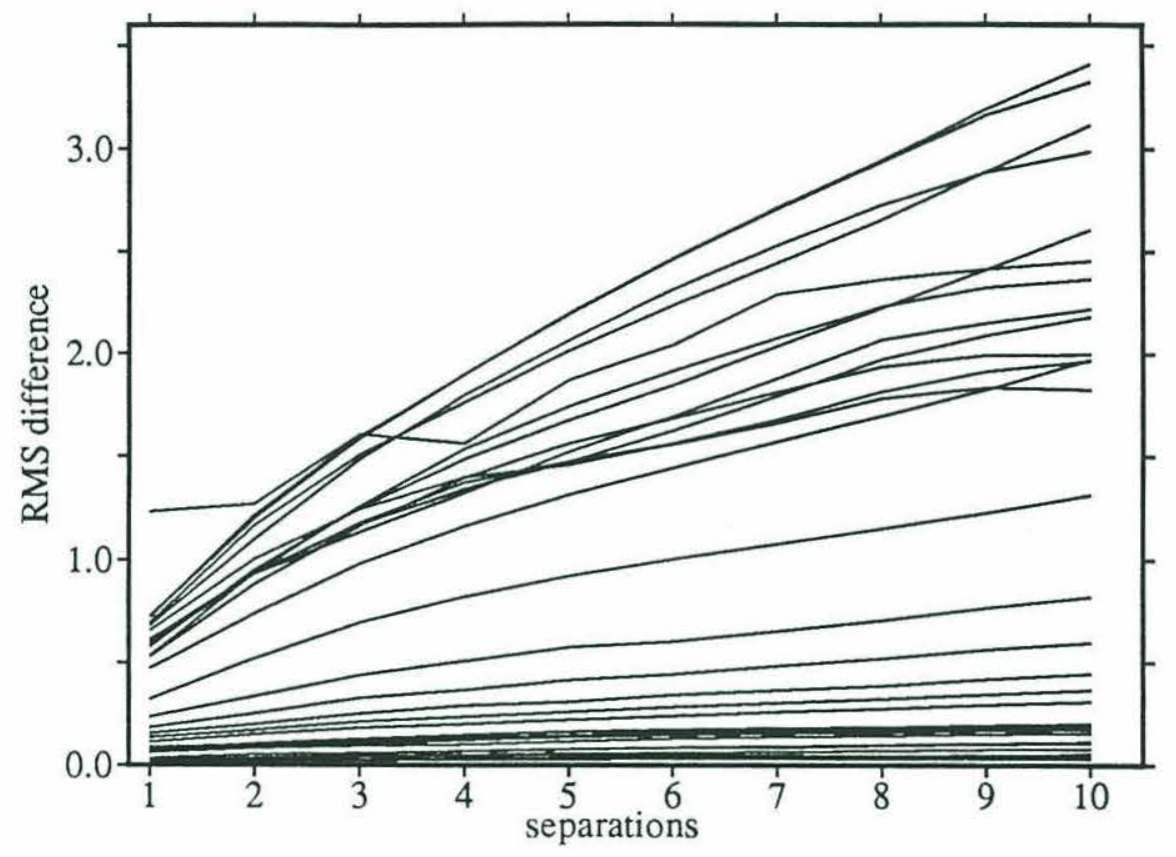

B.

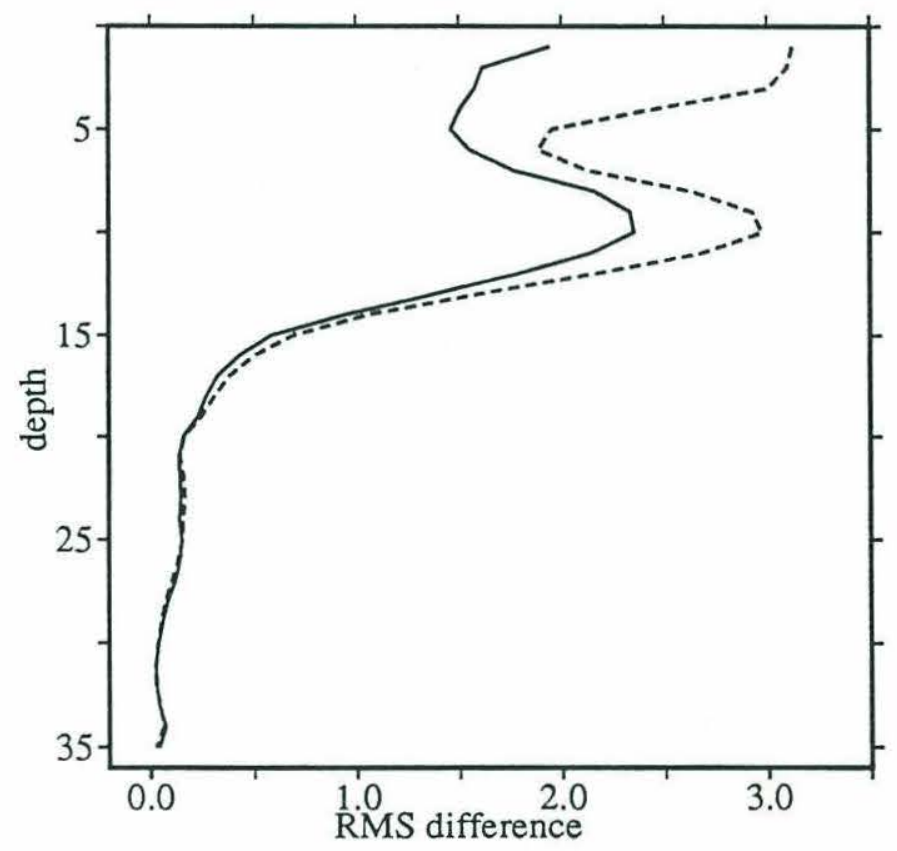

Figure 1-3. RMS separation difference of temperature $\left({ }^{\circ} \mathrm{C}\right)$

A. The RMS separation difference as a function of different station separation. The different curves correspond to the 35 standard depths.

B. Mean RMS separation difference of above A (solid) and the square root variance of data (dashed) vs depth. 
interior), we will use the RMS difference as a function of number of station separations, rather than distance in $\mathrm{km}$.

Figure 1-3 shows the RMS difference of temperature as a function of station separation for the first 35 standard depths. (There is only one station that is deeper than depth 35 for the Endeavor cruise 129.) The RMS difference increases with separation in general, which is probably due to picking up some of the signal variance of longer spatial scale. Also shown are the averages from one separation to ten separations of Figure 1-3a, and the RMS data variances about the mean. The average is much smaller than the data variance at shallower depths but becomes comparable at depth. We shall use this average RMS difference as a measure of noise, $\sigma_{i}$ in equation (1-6). This estimate is probably a pessimistic one, since it averages from the mean difference over ten station pairs, but should provide a reasonable estimate of the relative variance of noise among the different variables. This average has a value almost the same as the mean difference over five station pairs. The mean distance over five station separations for this particular hydrographic section is $170 \mathrm{~km}$. (At three station separations, the mean distance is $100 \mathrm{~km}$ and the RMS difference is $3 / 5$ the value of five station separations.)

Figure 1-4 shows the mean plus/minus the $\sigma_{i}$ profiles of the six variables. Now we may form the matrix $A$ with the columns being the different stations and different normalized tracers, and the rows being the standard depths.

$$
\begin{aligned}
A_{35 \times(80 \times 6)} & =(T, S, O, P, S i, N) \\
T_{35 \times 80} & =\left(\underline{t}_{1}, \underline{t}_{2}, \ldots, \underline{t}_{80}\right) \quad \text { etc. }
\end{aligned}
$$

The singular vectors of $A$ in equation (1-10) are what are called common principal components (Jolliffe, 1986), which represent common structures among different data. $\underline{t}_{i}$ is temperature at station $i$. In our present data set we have 80 stations and 35 standard depths. Any flagged data or any depth below the ocean floor was given the mean value, which in the normalized form is zero (equation 1-6); i.e. there is no signal 
A. temperature $(\operatorname{deg} \mathrm{C})$

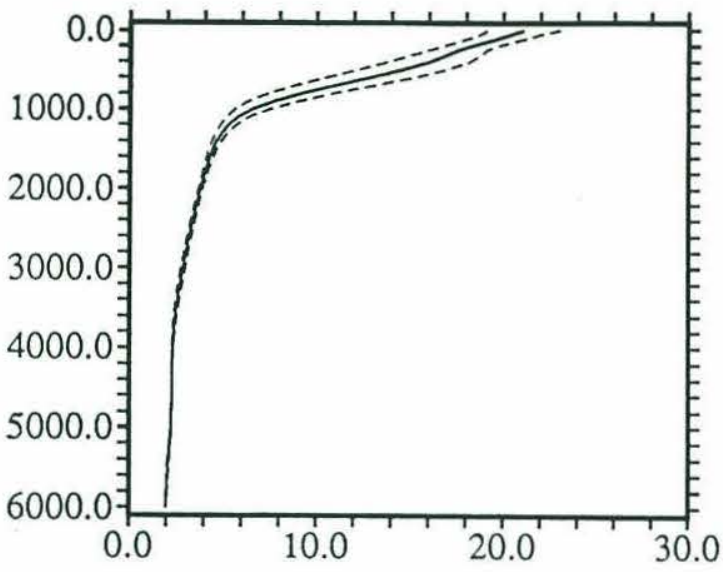

C. oxygen (m1/l)

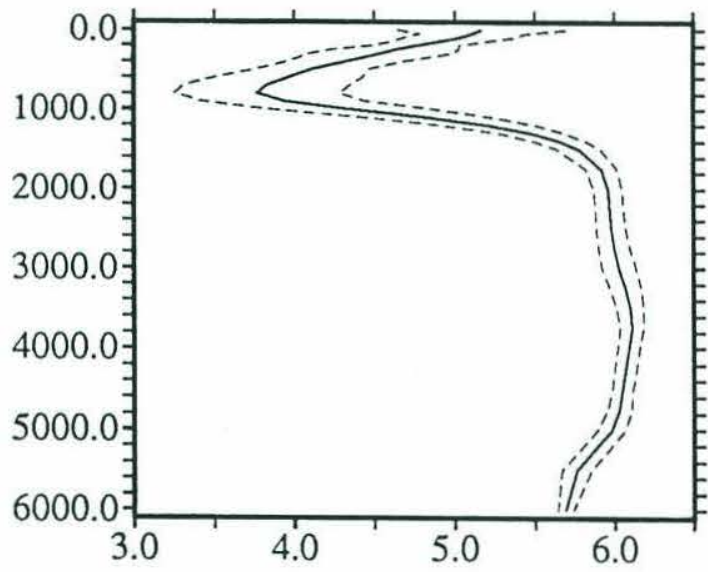

E. silicate (micro $\mathrm{mol} / \mathrm{kg}$ )

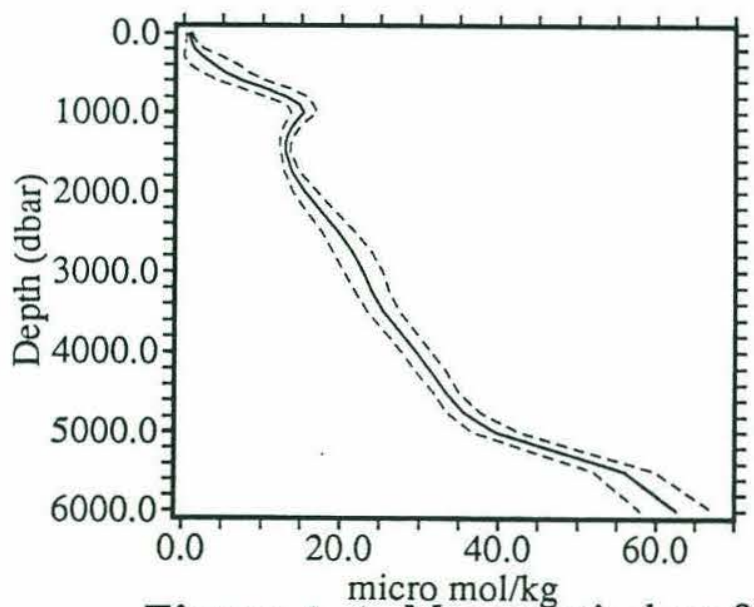

B. salinity (PSU)

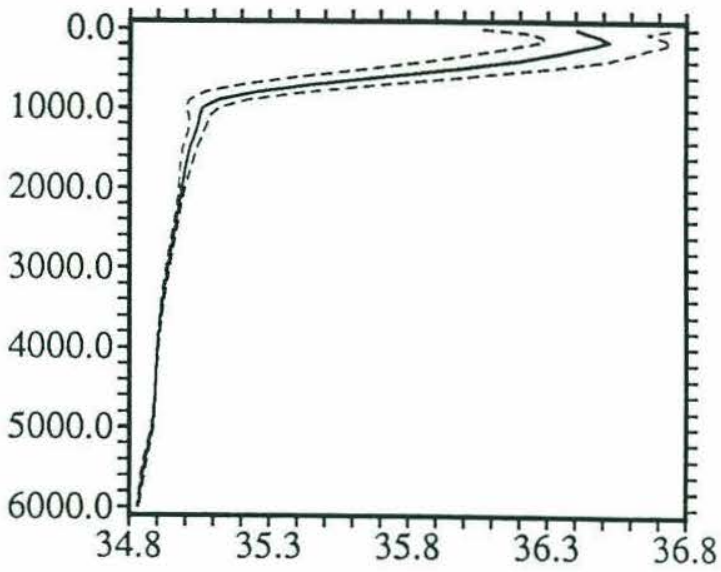

D. phosphate (micro $\mathrm{mol} / \mathrm{kg}$ )

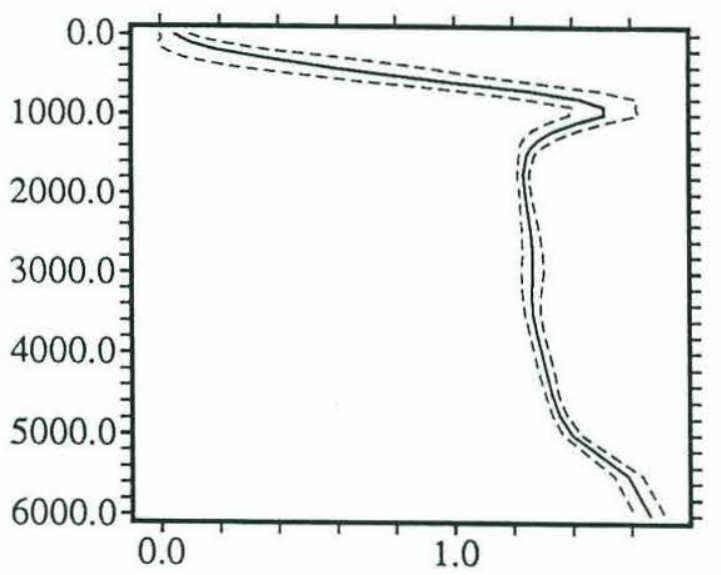

F. nitrate (micro $\mathrm{mol} / \mathrm{kg}$ )

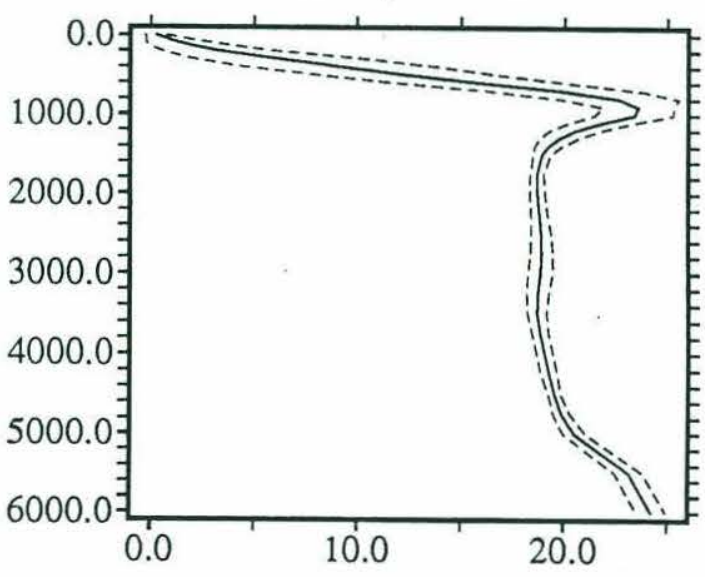

Figure 1-4. Mean vertical profiles plus/minus normalization, $\sigma_{i}$.

(a) Temperature $\left({ }^{\circ} \mathrm{C}\right)$ (b) Salinity (practical salinity units)

(c) Oxygen $(\mathrm{ml} / \mathrm{l})(\mathrm{d})$ Phosphate $(\mu \mathrm{mol} / \mathrm{kg})$

(e) Silicate $(\mu \mathrm{mol} / \mathrm{kg})$ (f) Nitrate $(\mu \mathrm{mol} / \mathrm{kg})$ 
to explain below the ocean floor. By using many stations in calculating the modes that do have the full depth profile, the effect of this sudden change at the ocean floor, i.e. going from non-zero to zero, may be negligible.

The vertical modes for this matrix are shown in Figure 1-5. The first vertical mode has large values above $1000 \mathrm{~m}$ (standard depth 14, Table 1-1), while the second has large values below this depth. This would indicate that the first mode will account for the mean vertical variation above $1000 \mathrm{~m}$ and the second below that depth. The higher modes have increasingly smaller vertical scales as evidenced by the increasing number of zero crossings in the vertical.

Horizontal modes are plotted in Figure 1-6 for each separate variable. We can associate some of the structures in $\underline{v}_{i}$ with the hydrography. The large values towards the northern end of mode 1 correspond to stations north of the Gulf Stream and the monotonic change in mode 2 of the nutrients is indicative of the broad scale north south gradient at depth. We shall see some of what the modes account for in the hydrographic structures later in Section 5. Note that some of the $\underline{v}_{i}$ 's are nearly identical from scalar to scalar. This reflects the similarities we saw in the horizontal variations of the original section contours (Figure 1-2). This aspect of the modes will be discussed in Section 6.1. One more feature that catches the eye is that the higher modes appear to have more short scale wiggles than lower modes. This indicates that the higher vertical modes are associated with short scale horizontal fluctuations of the hydrographic structures.

The singular values decrease exponentially with mode number (Figure 1-7). From these values, the variance accounted for by each mode is shown in Table 1-2 together with the percentage variance up to the sum of each mode. More than $80 \%$ of the total variance is accounted for by the sum of the first four modes. In the next section, we shall analyze how many modes we need to reproduce the data adequately, as defined earlier in this section. 


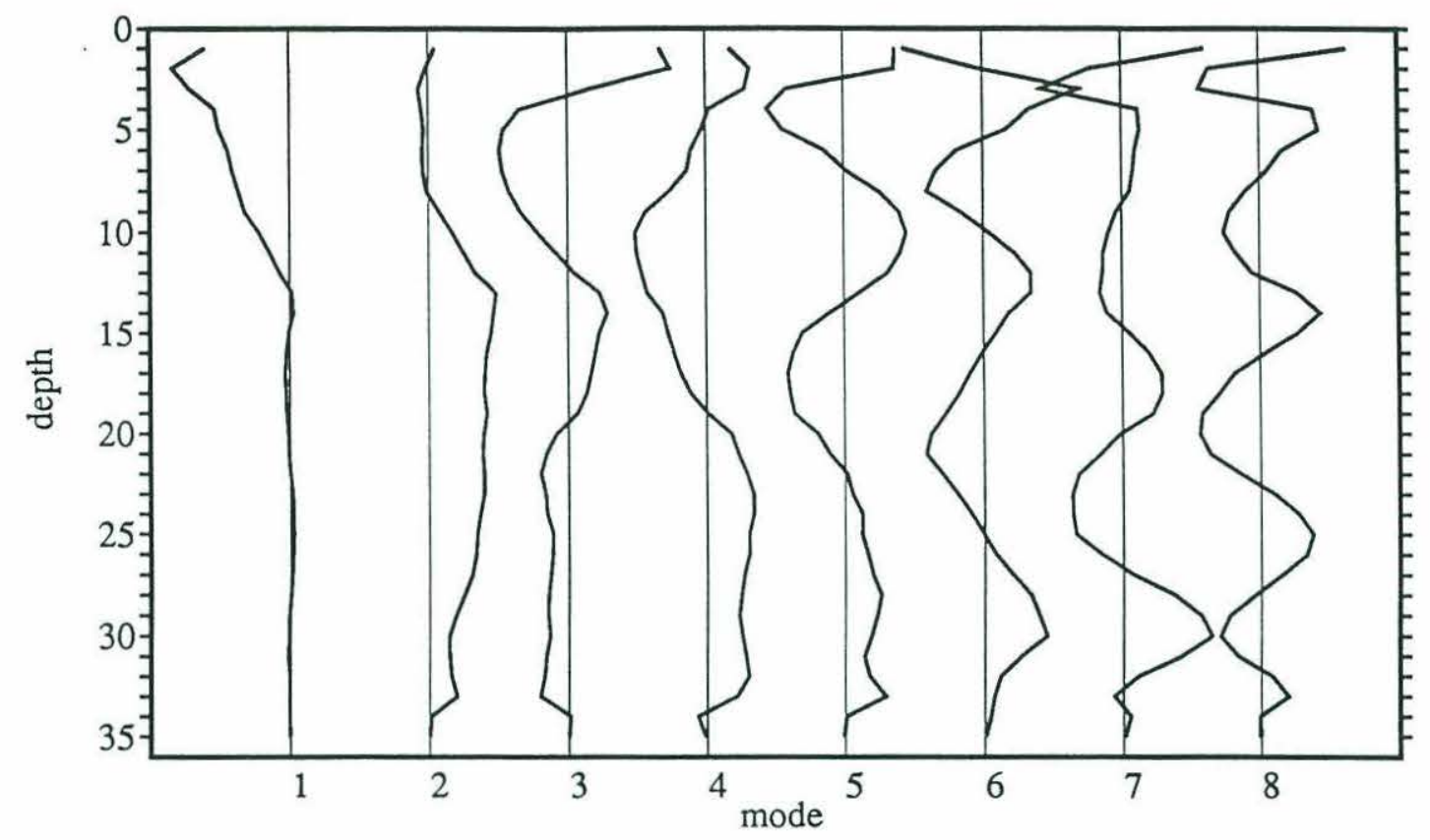

Figure 1-5. Vertical Modes.

These are the first 8 singular vectors, $\underline{u}_{i}$. The vertical axis is standard depth.

a. Temperature Expansion Coefficients

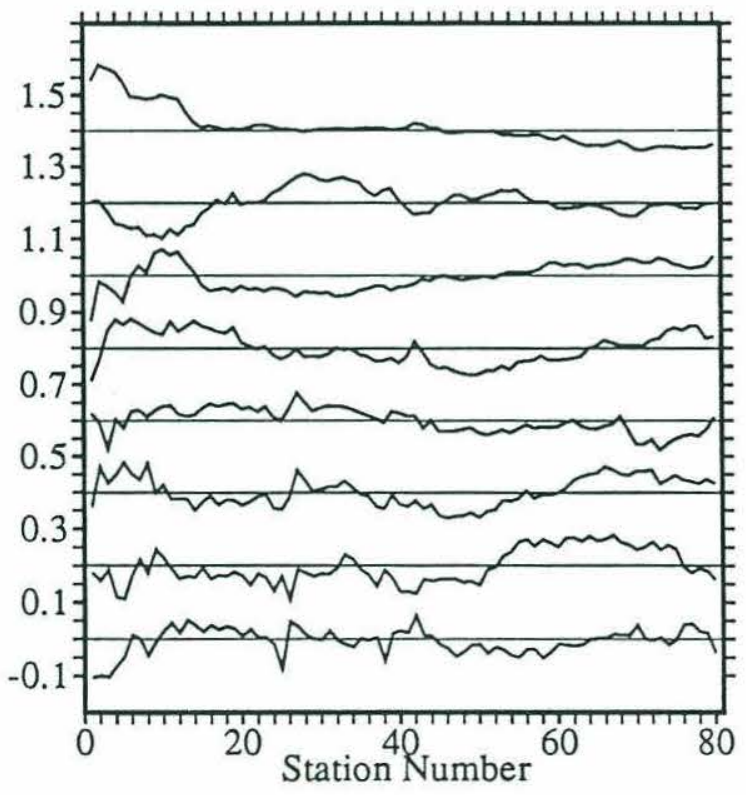

b. Salinity Expansion Coefficients

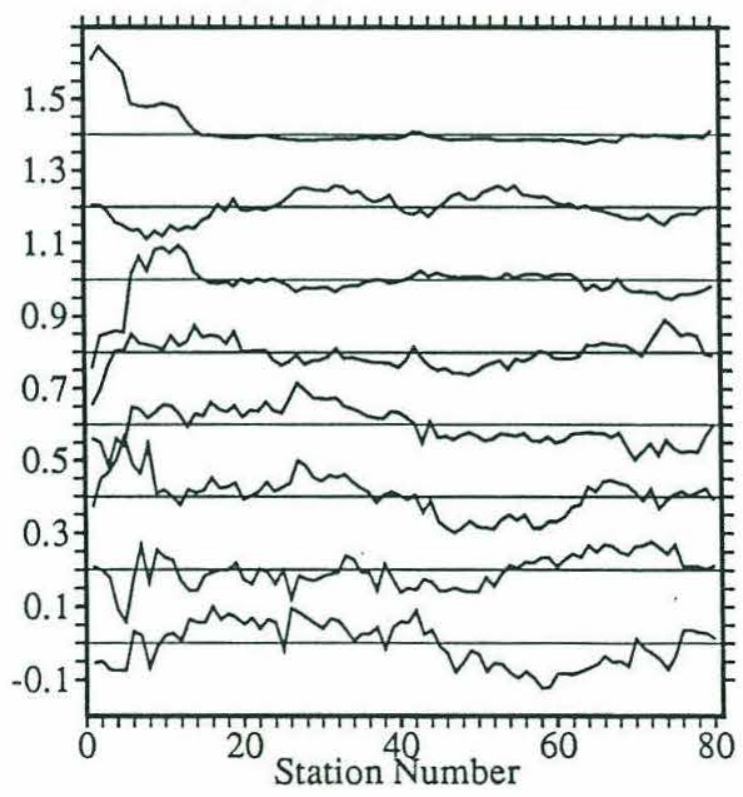

Figure 1-6. Elements of Row Singular Vector.

The top most curve in each variable is the first singular mode with subsequent modes offset downward. The northern end of the section is towards the far left of each figure as in Figure 1-2.
(a) Temperature
(b) Salinity 
c. Oxygen Expansion Coefficients

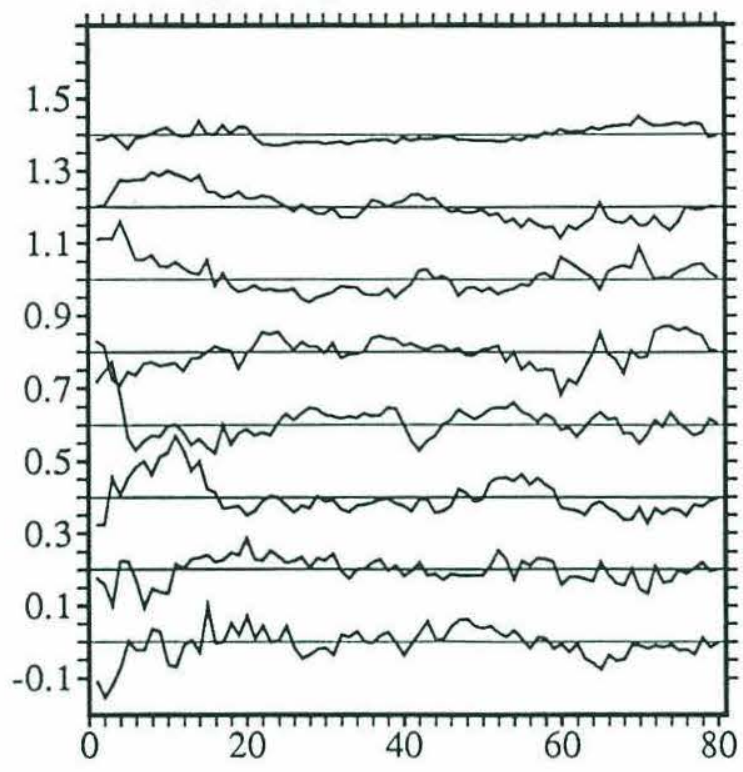

e. Silicate Expansion Coefficients

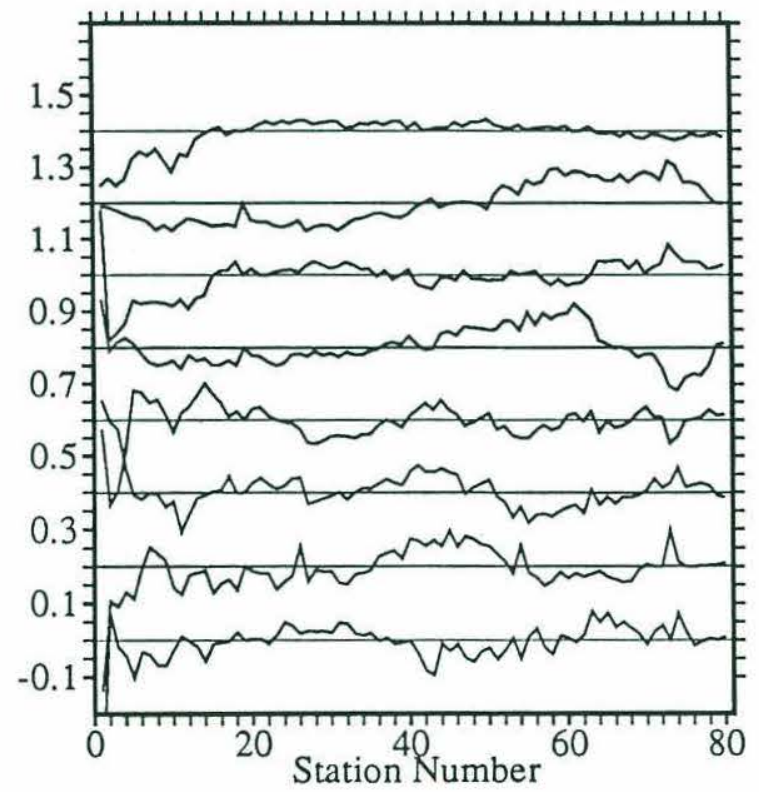

d. Phosphate Expansion Coefficients

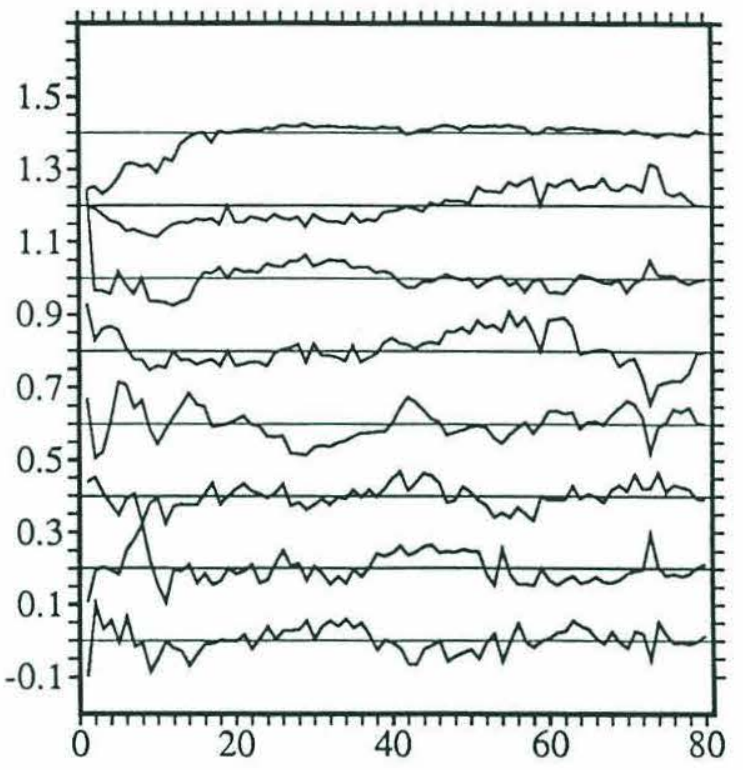

Figure 1-6. continued

(c) Oxygen (d) Phosphate (e) Silicate (f) Nitrate

f. Nitrate Expansion Coefficients

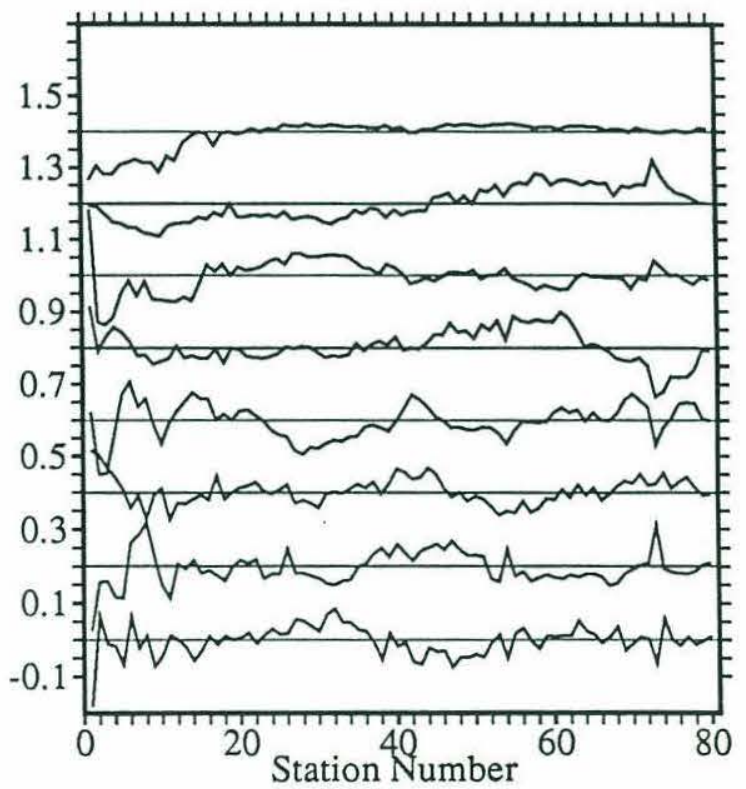




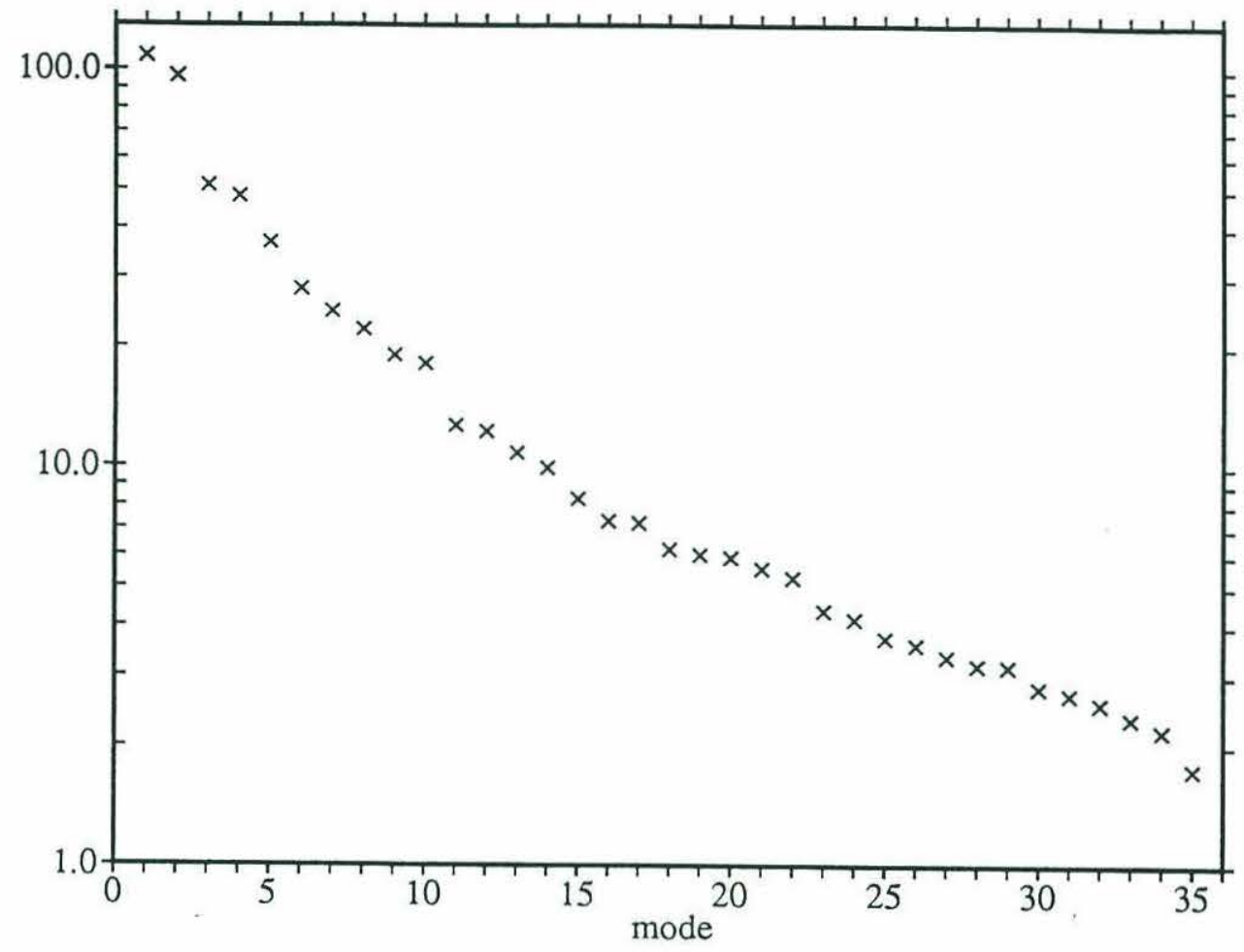

Figure 1-7. The Singular Values vs Mode Number

\begin{tabular}{|c|c|c|}
\hline mode & singular val. & percentage \\
\hline 1 & 108.0 & 37.9 \\
2 & 96.3 & 68.0 \\
3 & 50.9 & 76.5 \\
4 & 48.0 & 84.0 \\
5 & 36.7 & 88.4 \\
6 & 27.9 & 90.9 \\
7 & 24.5 & 92.9 \\
8 & 22.0 & 94.5 \\
9 & 19.0 & 95.6 \\
10 & 18.1 & 96.7 \\
\hline
\end{tabular}

Table 1-2. The first ten singular values and the cumulative percentage of variance reduction. 


\section{Determination of the Significant Degrees of Freedom}

We will now analyze the result to determine how many degrees of freedom there are to the large scale coherence. We have seen above that the first few modes can account for a substantial amount of the data variance. Requiring of four degrees of freedom would produce a factor of 9 in the amount of data reduction. (Four modes as opposed to 35 depths.) What we need to establish though is that the remainder is not part of the dominant features but could be considered as noise. The number of degrees of freedom will be denoted the "rank". This terminology should be distinguished from the mathematical rank (Section 4.1) of the matrix $A$ (equation 1-10). The usage of "rank" here and in the rest of this thesis is meant to be the "effective rank". It is the number of degrees of freedom required, and retained in a reduced description. For example, a rank $l$ reconstruction (approximation) of the data is a sum of the first $l$ terms in the expansion of equation (1-4a) (Section 4.1).

Table 1-3 lists the normalized RMS norm of the differences between the data and the modal reconstruction for each variable as a function of rank. The residuals quickly become small, until they become the same magnitude as or smaller than the error estimates. Also note that the reduction is nearly the same for any variable. In other words, each mode accounts for just about the same amount of variance among different variables. The first four modes makes the RMS residuals about a half of the weighting factor which is also about the same order of magnitude as the difference at one station separation of the data (Figure 1-3a). In Figure 1-8, we plot the same normalized RMS residuals of salinity and phosphate as a function of depth. The first mode quickly reduces the shallower residuals, while the second mode does that at deeper levels. With the inclusion of higher modes, the residual profiles become nearly depth invariant. This would mean that the modes are accounting for the variations more or less uniformly with depth. 


\begin{tabular}{|c|c|c|c|c|c|c|}
\hline rank & $\mathrm{T}$ & $\mathrm{S}$ & $\mathrm{O}$ & $\mathrm{P}$ & $\mathrm{Si}$ & $\mathrm{N}$ \\
\hline 0 & 1.478 & 1.593 & 1.172 & 1.514 & 1.615 & 1.424 \\
1 & 1.000 & 1.048 & 1.107 & 1.155 & 1.354 & 1.164 \\
2 & 0.680 & 0.819 & 0.774 & 0.764 & 0.900 & 0.791 \\
3 & 0.590 & 0.675 & 0.654 & 0.685 & 0.779 & 0.682 \\
4 & 0.479 & 0.614 & 0.556 & 0.524 & 0.634 & 0.545 \\
5 & 0.424 & 0.522 & 0.482 & 0.437 & 0.538 & 0.447 \\
6 & 0.367 & 0.440 & 0.405 & 0.402 & 0.447 & 0.401 \\
7 & 0.302 & 0.397 & 0.376 & 0.358 & 0.366 & 0.349 \\
8 & 0.270 & 0.318 & 0.336 & 0.333 & 0.319 & 0.318 \\
\hline
\end{tabular}

Table 1-3. RMS Nondimensional Residuals

These are the normalized root mean square residuals of each variable as a function of number of retained modes, i.e. rank. (Rank 0 corresponds to the normalized data norm.)
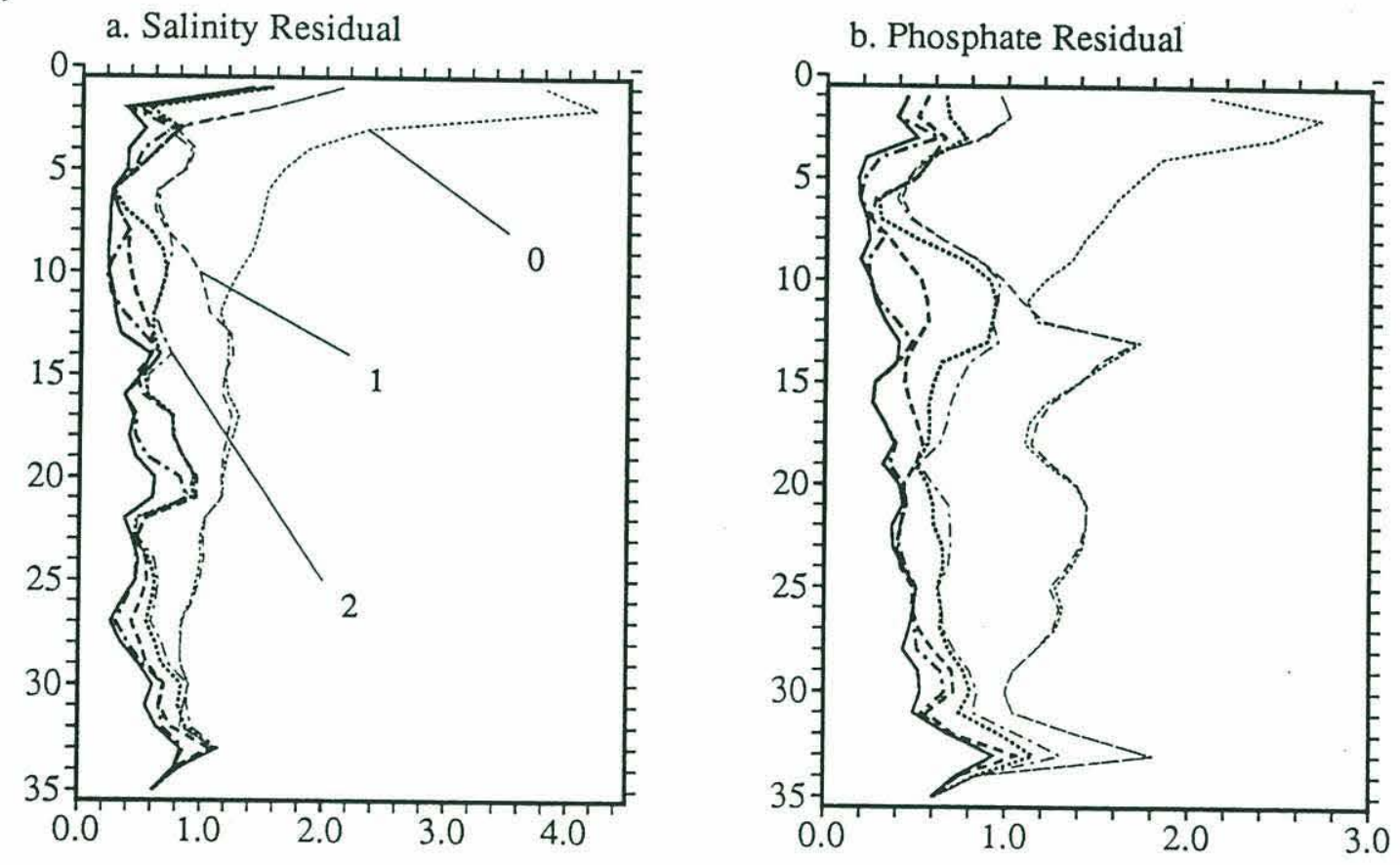

Figure 1-8. RMS Nondimensional residual vs depth.

(a) Salinity (b) Phosphate

This plots the RMS residual profile as a function of rank. The rank for the thin dotted curve is 0 , dashed is 1 , chain-dashed is 2 , thicker dotted is 3 , thicker dashed is 4 , thicker chain-dashed is 5 and thicker solid curve is 6 . 
Let's now examine the actual reconstructed fields. In Figures 1-9 and 1-10, we plot the temperature and silicate fields respectively. There are six representations of the section for each variable, corresponding to the first mode reconstruction (rank 1) up to the sum of the first six modes (rank 6). Even with only the first two modes (rank 2 ), we see the gross nature of the section reproduced. The first mode brings up the Gulf Stream signal, and the second mode reproduces the broad north south gradient in silicate, just as we envisioned from the structure of the $\underline{v}_{i}$ vectors (Figure 1-6). Higher modes bring up the finer details of the section, and by rank six, the reconstructed field is nearly indistinguishable from the data to the eye.

As another example, in Figure 1-11, we make a comparison between the salinity data and the rank 4 salinity reconstruction at $600 \mathrm{~m}$ (standard depth 10). Also shown is the size of the normalization $\sigma_{i}$ (equation 1-6). All the major features like the bowl shape over stations 10 to 73 , and the cold core ring centered at about station 38 are reproduced within the measure of noise: the residuals are about the same magnitude as the much smaller short scale variations seen in the data.

To further quantify the goodness of the modal reconstruction, we next compare the total zonal transports. While the property fields are contaminated by eddies, transports associated with them could be expected to nearly average out when integrated meridionally. It is also important that the modal reconstruction reproduce the large scale transports if it is to be useful, since it is these transports that define the ocean circulation. The velocities were calculated geostrophically with a uniform reference level at $2000 \mathrm{~m}$. The density distribution is constructed from temperature and salinity of the original data and the modally reconstructed results as discussed above. Because we are only interested in the integrated property difference (from one end of the section to the other) between the original data and the modal reconstructions, the exact reference level velocity does not matter as long as we use the same one. When the 

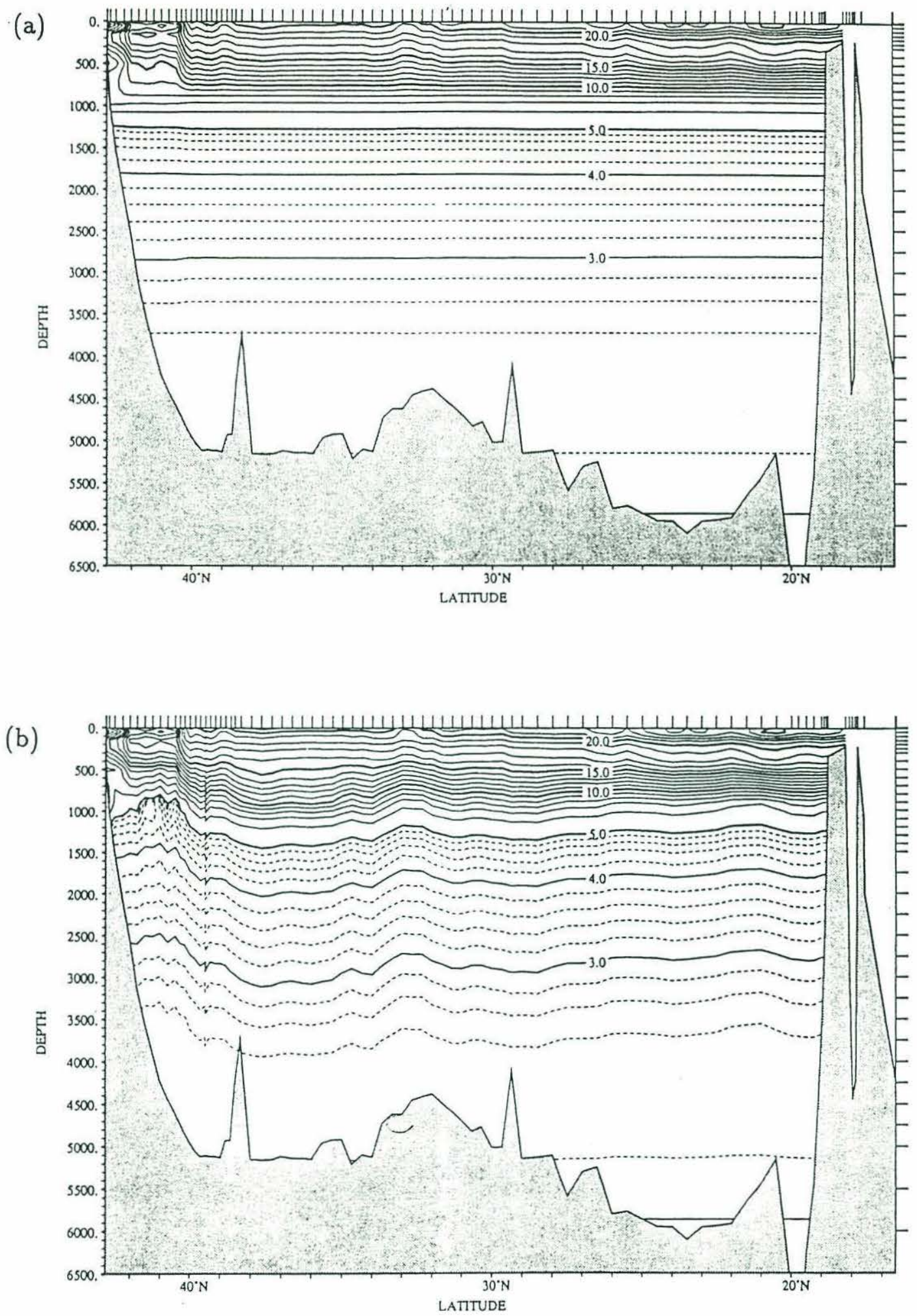

Figure 1-9. Modally Reconstructed Temperature Distribution. (a) rank 1, (b) rank 2 

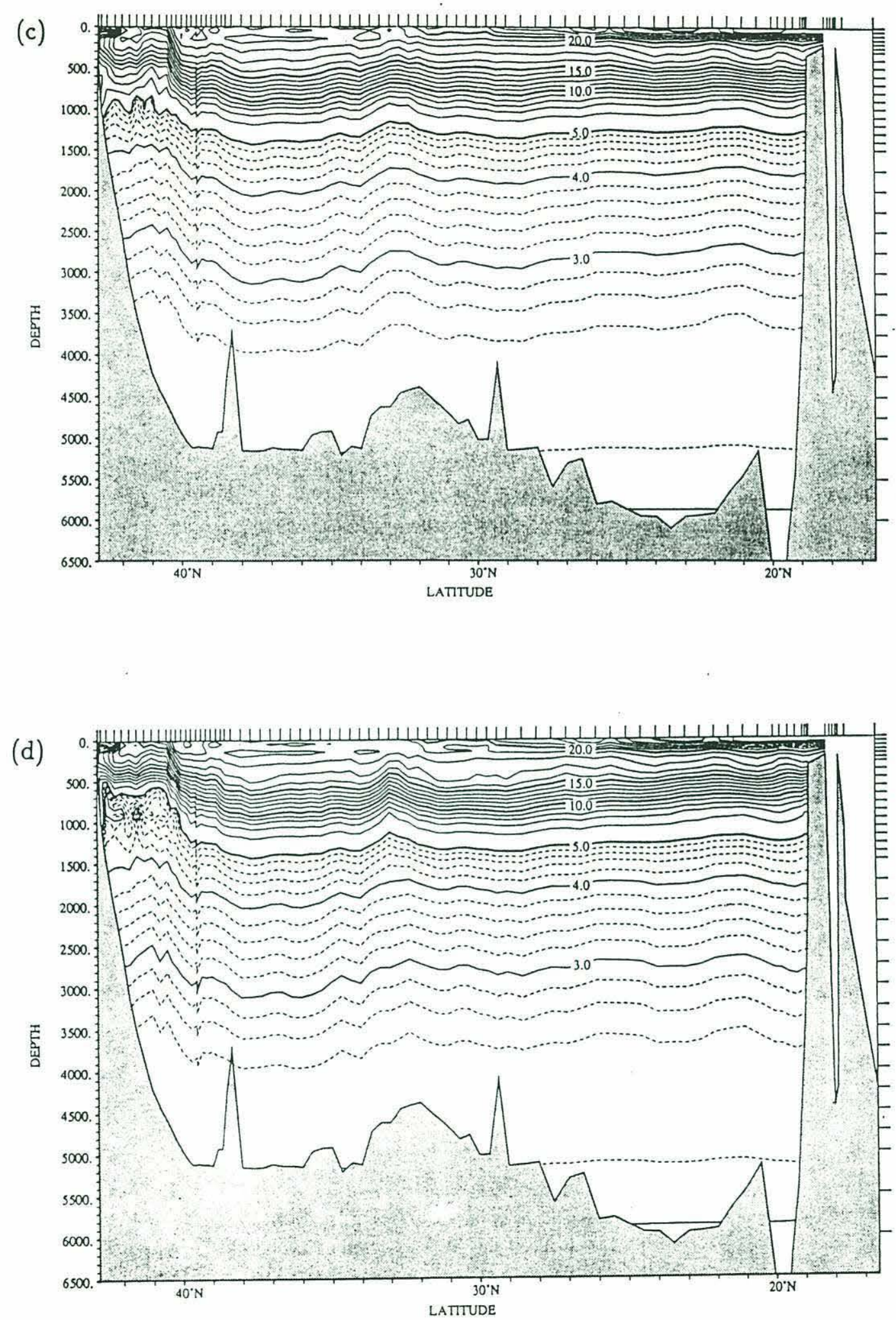

Figure 1-9. (continued) (c) rank 3, (d) rank 4 

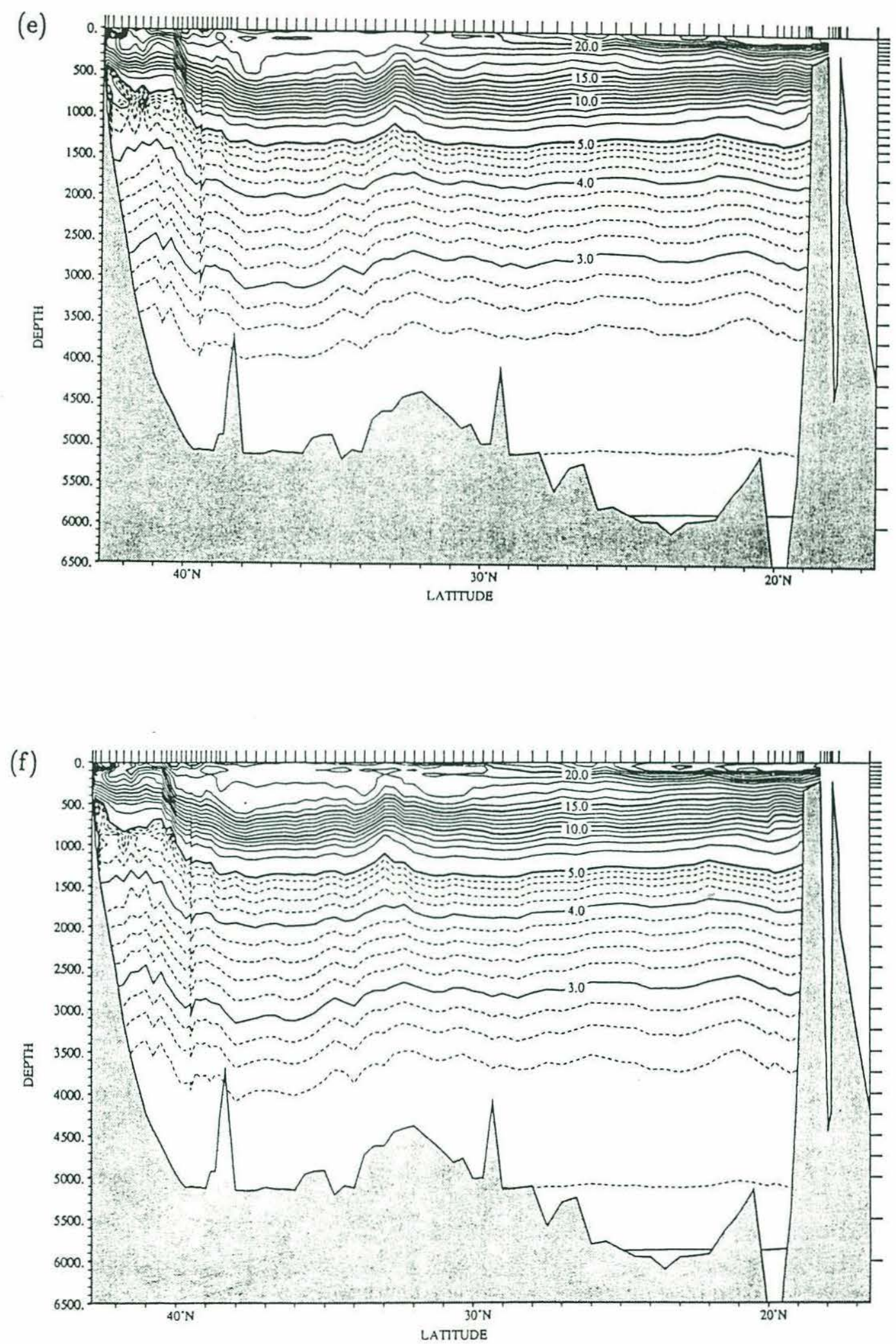

Figure 1-9. (continued) (e) rank 5, (f) rank 6 

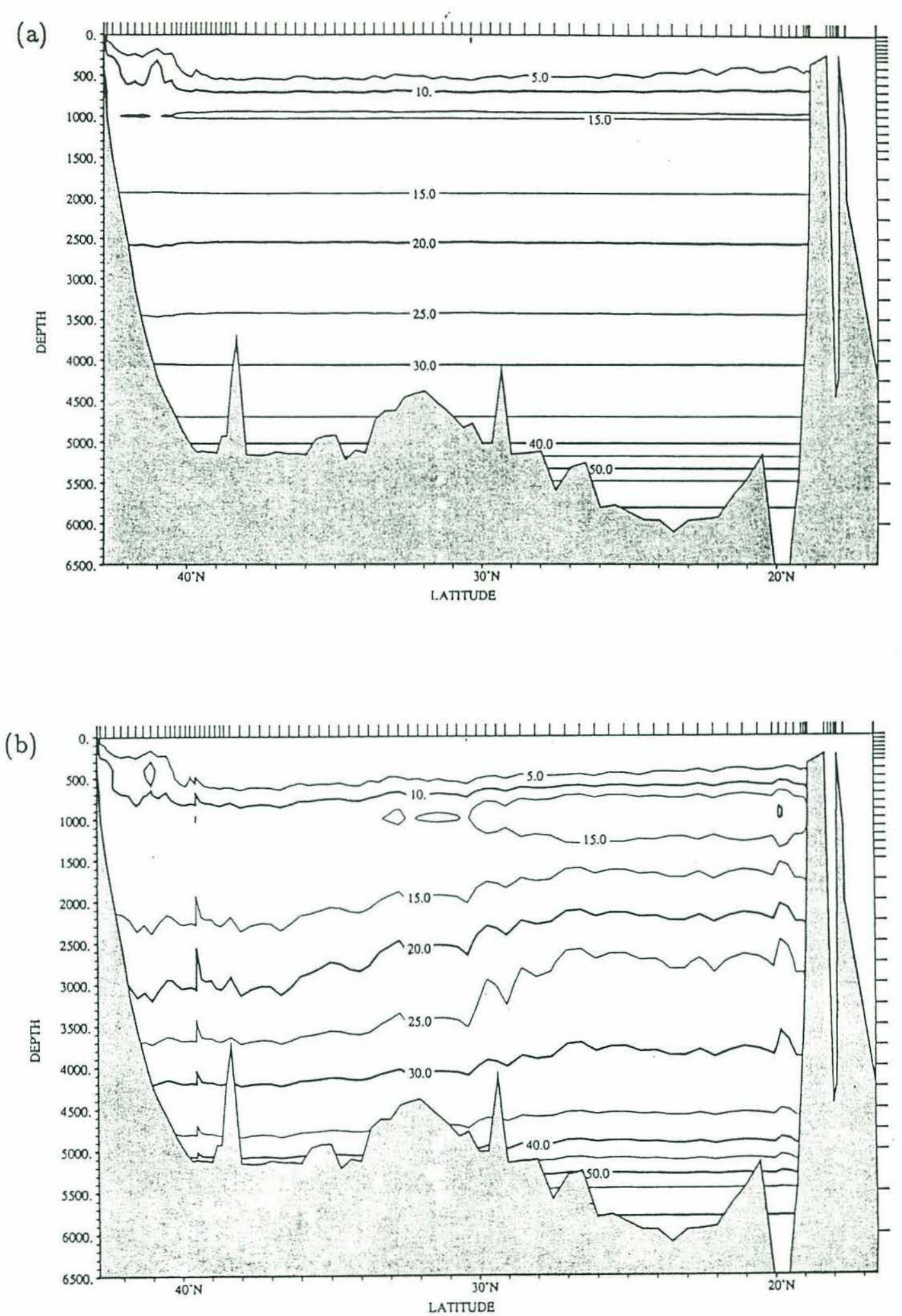

Figure 1-10. Modally Reconstructed Silicate Distribution. (a) rank 1, (b) rank 2 

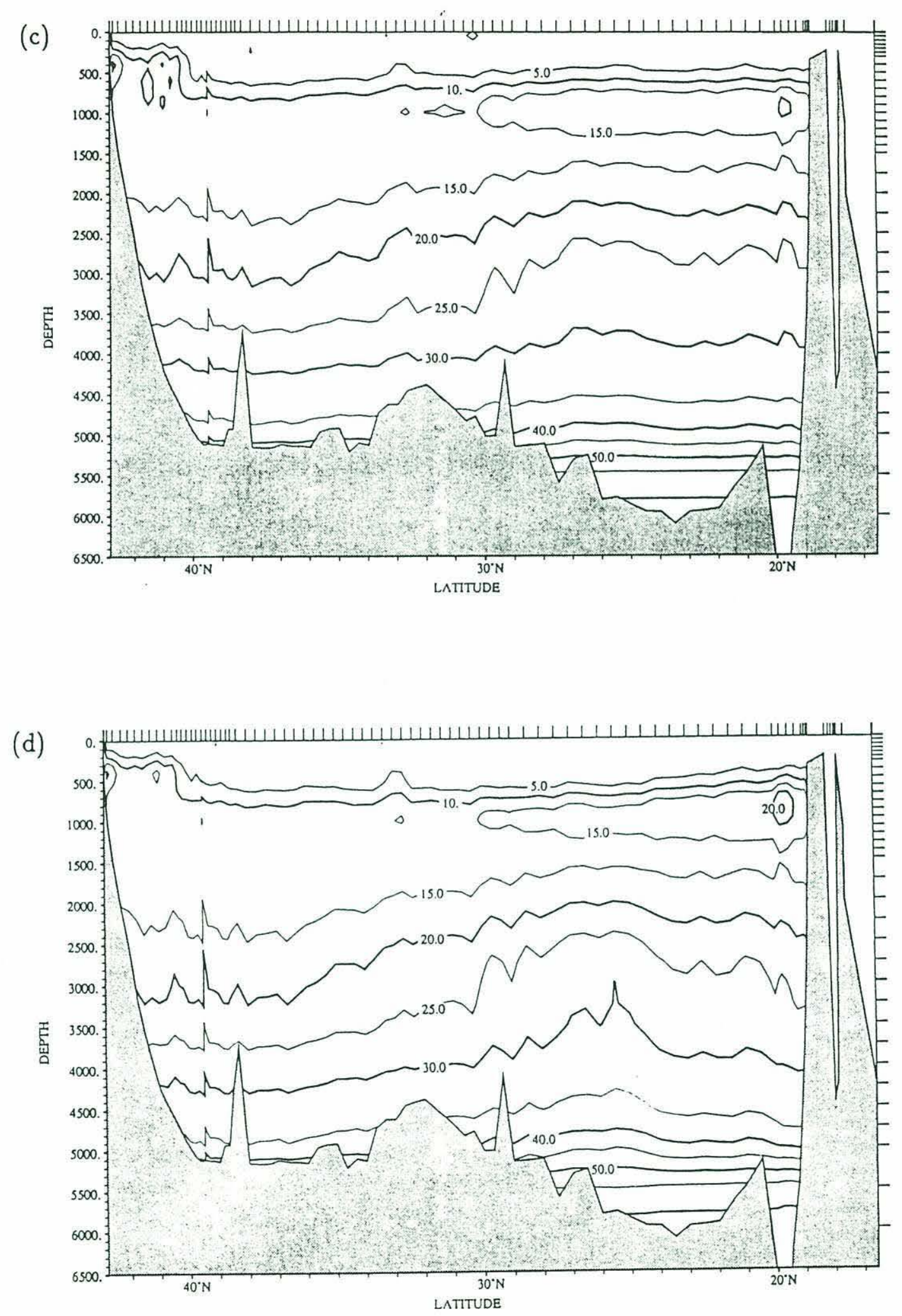

Figure 1-10. (continued) (c) rank 3, (d) rank 4 


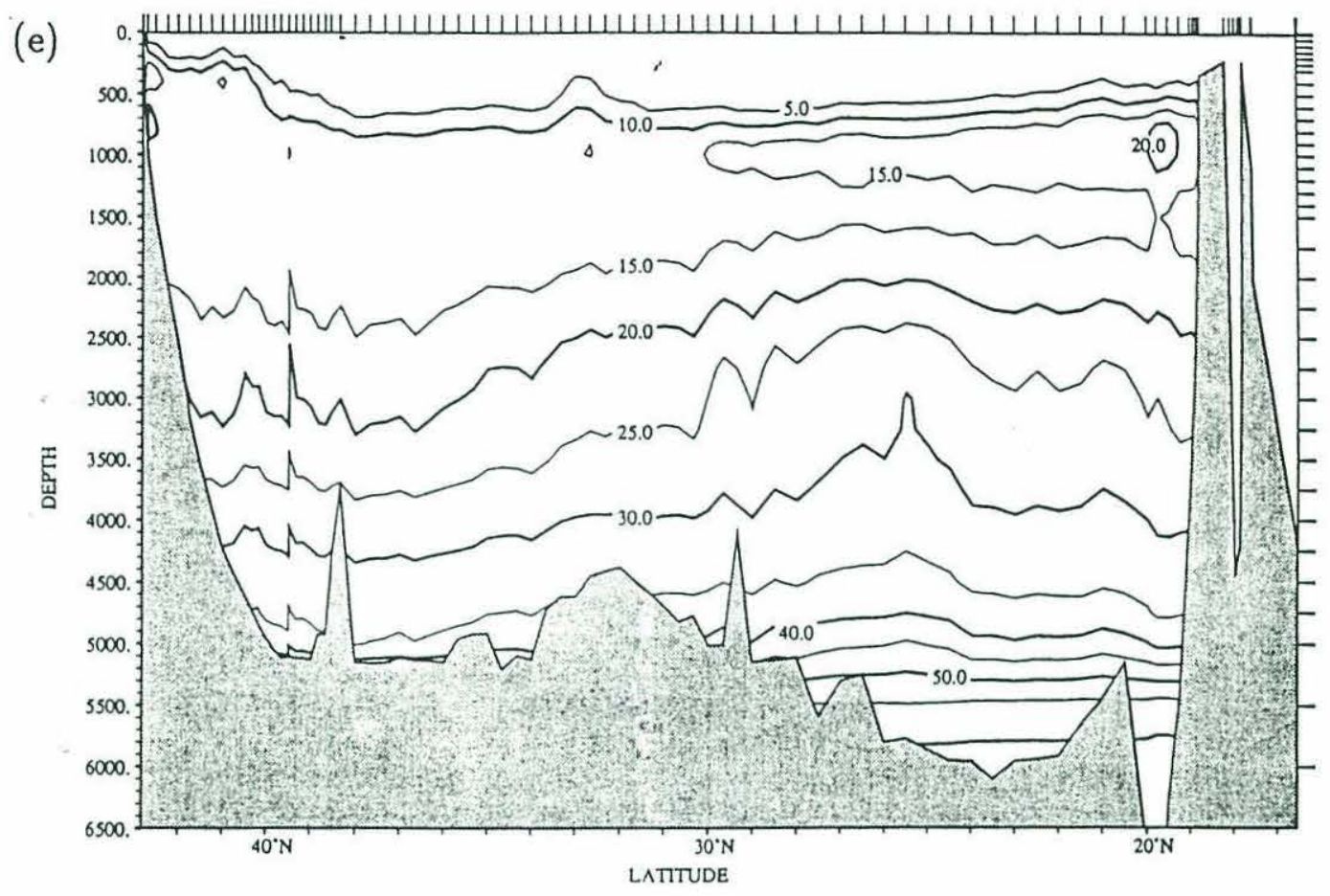

(f)

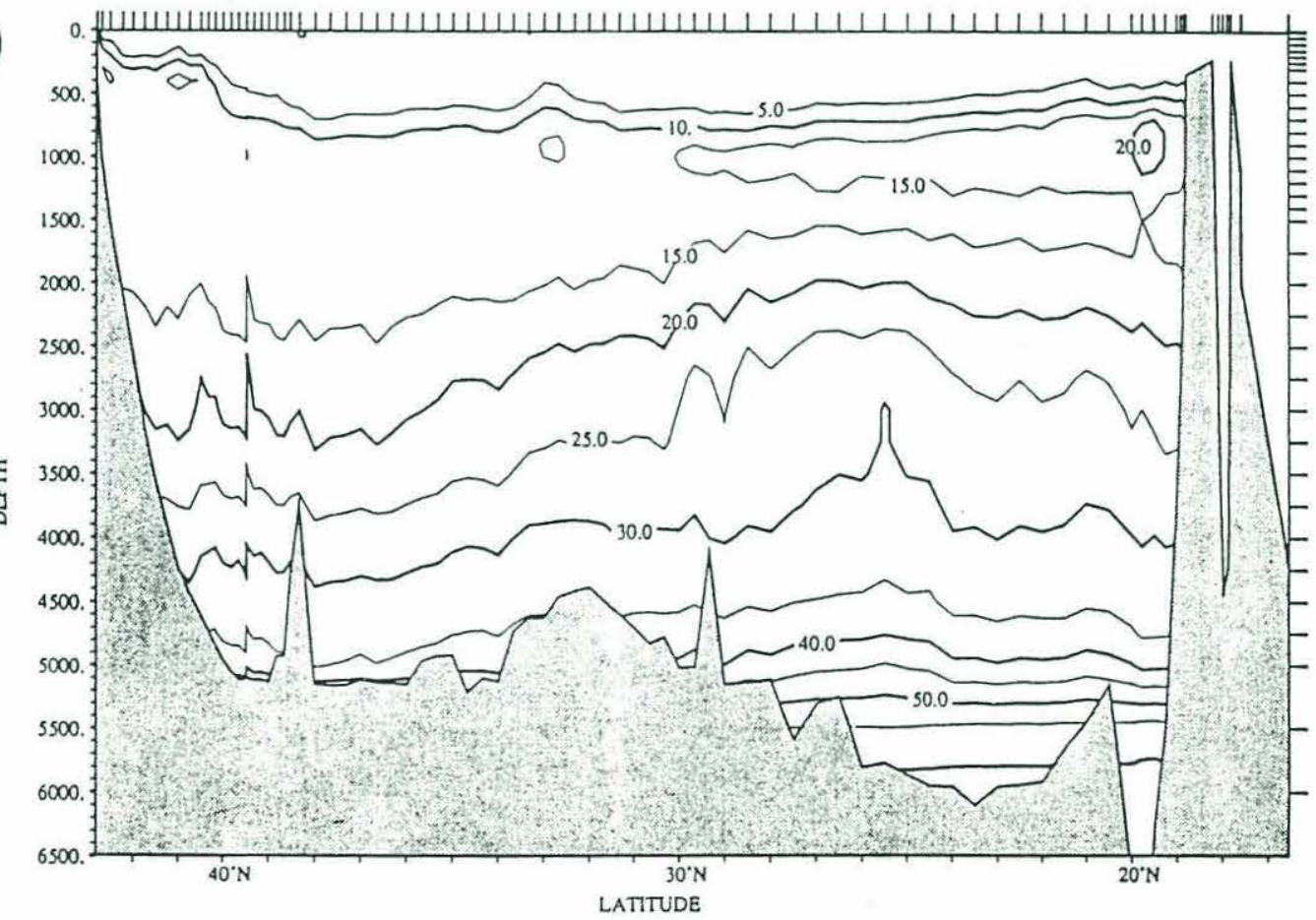

Figure 1-10. (continued) (e) rank 5, (f) rank 6 


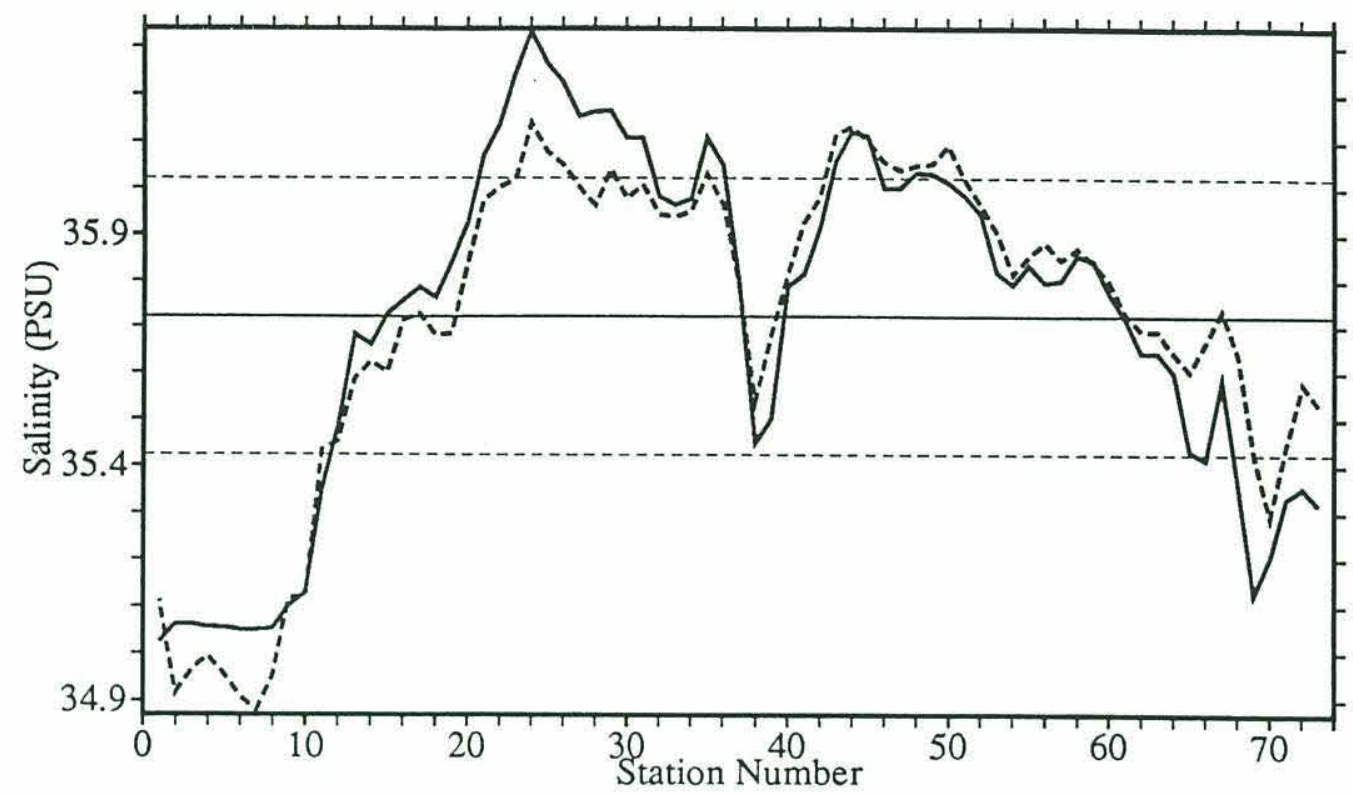

Figure 1-11. Salinity at $600 \mathrm{~m}$

Solid curve is data and the dashed curve is modal reconstruction at rank 4 . The extreme left corresponds to the northern end of the section as in Figure 1-6. The base line at 35.72 is the mean salinity at this depth, and the two dashed lines about this are this mean plus/minus the normalization, $\sigma_{i}$.

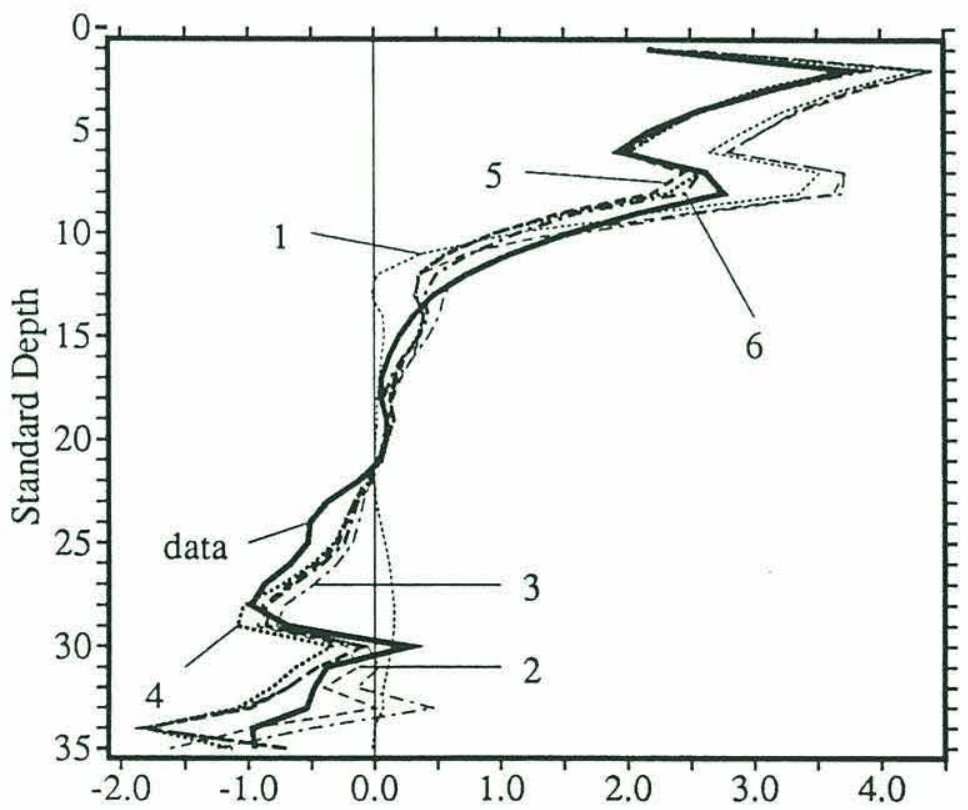

Figure 1-12. Mass Flux (Sv) at Each Standard Depth.

Geostrophic velocity is referenced at $2000 \mathrm{~m}$, positive eastward. Solid curve is the flux associated with the data, while the others are flux at different ranks. The thin dotted curve is rank 1, dash rank 2, chain-dash rank 3, thicker dotted curve rank 4, thicker dash rank 5 and the thicker chain-dash is rank 6. 
stations become shallower than the uniform reference surface, the geostrophic velocity was referenced to the shallower of the station pair.

Figure 1-12 shows the mass transport estimates as a function of depth. Mass flux is calculated between layers, which boundaries are defined as the mid-depths between the standard depths (Table 1-1). Mass flux between any pair of station is calculated down to the common maximum standard depth. Even with just two modes, the gross nature of the total flux is evident. There is a net eastward flow above the reference level and westward flux below. Note that mode 1 can reproduce most of the mass flux above $900 \mathrm{~m}$ (standard depth 13), while mode 2 resolves the lower half. There appears to be a sudden improvement at mode 4 above $400 \mathrm{~m}$ (depth 8), while not much of a difference is obvious beyond this rank. At rank 4 (6), the net transport is $15.8 \mathrm{~Sv}$ (18.8 Sv) to the east while the data has $20.1 \mathrm{~Sv}$ to the east.

Figure 1-13 is a plot of transport between individual station pairs in a layer $100 \mathrm{~m}$ thick centered at $600 \mathrm{~m}$ (depth 10). The two curves are the data and the reconstruction at rank 4. The difference at any pair is generally smaller than the transport itself. The transport between any station pair is dominated by short length scale fluctuations and any large scale circulation is not readily apparent. However, if we take the cumulative sum of the transport, we can start to pick out the large scale trend (Figure 1-13). The net transport becomes eastward between station pairs 11 to 27 where we cross the Gulf Stream. The transport south of this is predominantly westward as seen as the general decrease of the total cumulative flux. The cold core ring appears as a spike at about station number 38. Notice that the residuals at strong currents, such as this cold core ring or the Gulf Stream, are not any larger than the rest of the section. The difference between the fluxes appears to be random, and in fact the cumulative difference from the northern end of the section shows the total difference is less than the meso-scale fluctuations and thus not systematic. 
a.

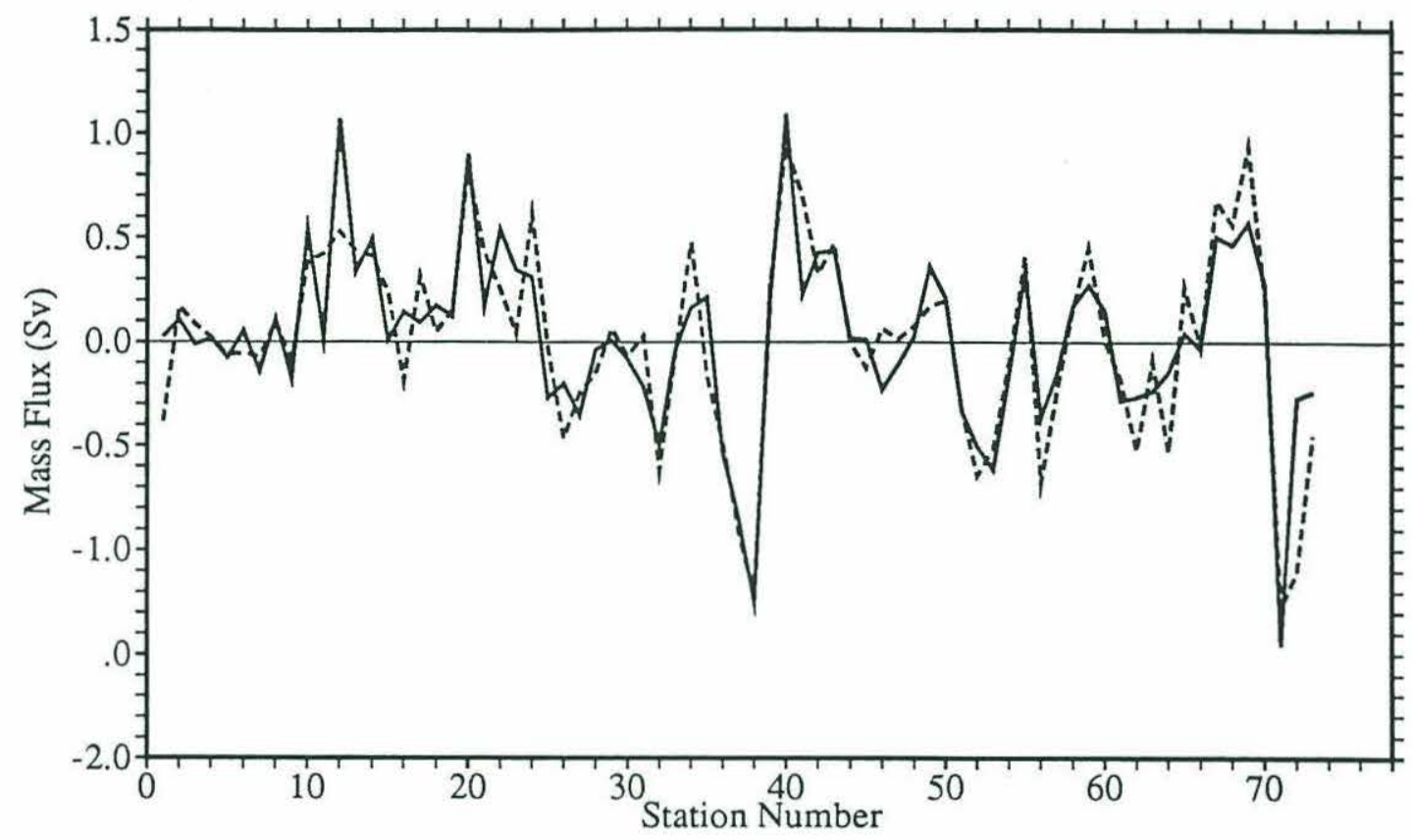

b.

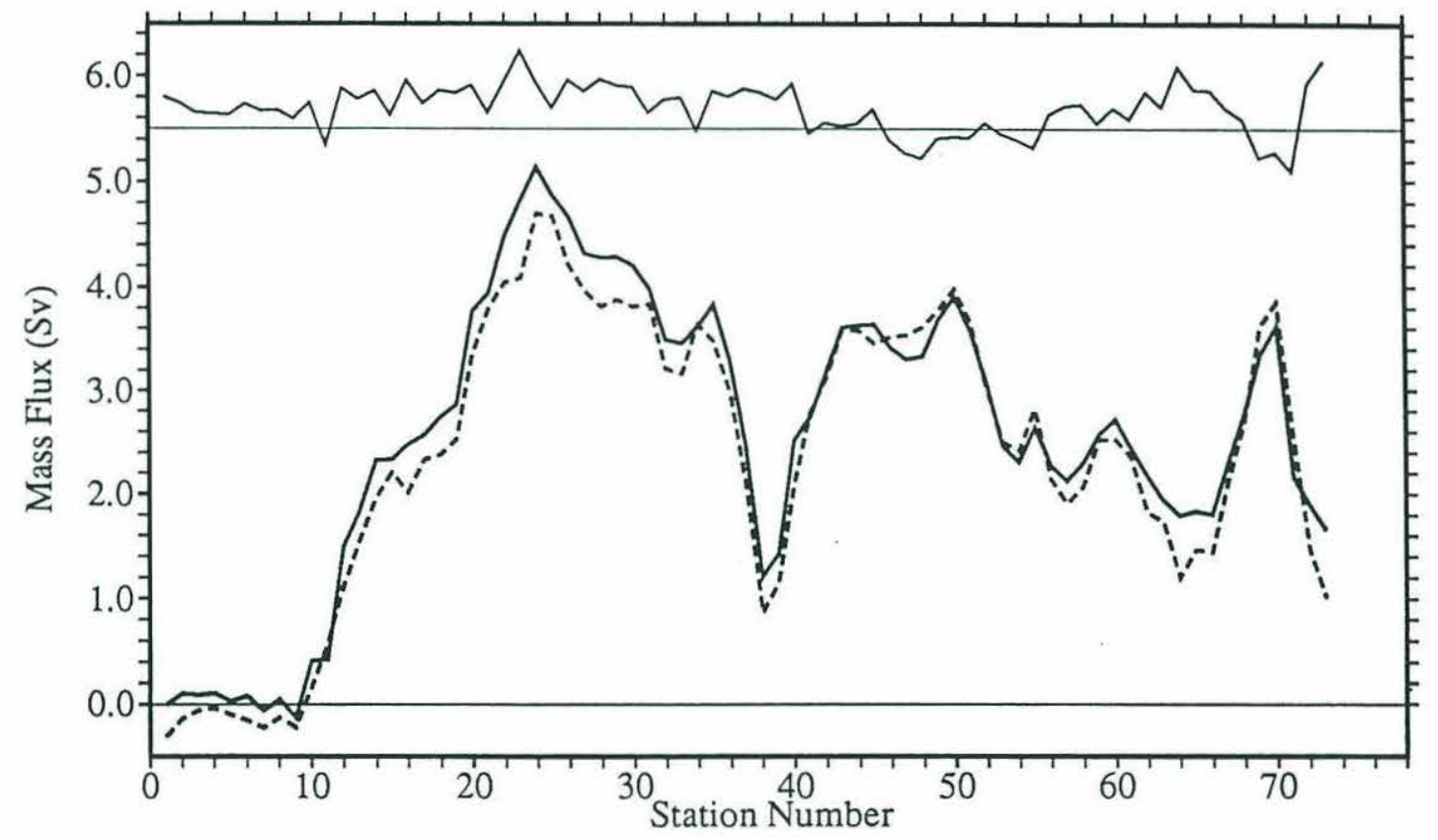

Figure 1-13. Mass Flux (Sv) in a Layer Centered at $600 \mathrm{~m}$

(a) Mass flux between each station pair.

(b) Cumulative mass flux from the northern end.

The extreme left is the northern end of the section as in Figure 1-6. The solid curve is the data and the dashed curve is the result at rank 4 . The difference of the cumulative flux is plotted in (b) offset (zero line at $5.5 \mathrm{~Sv}$ ) to the top. 
a.

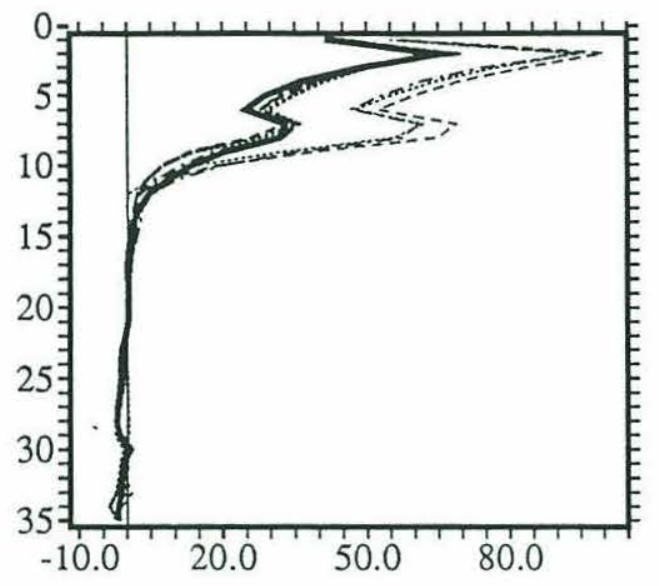

c.

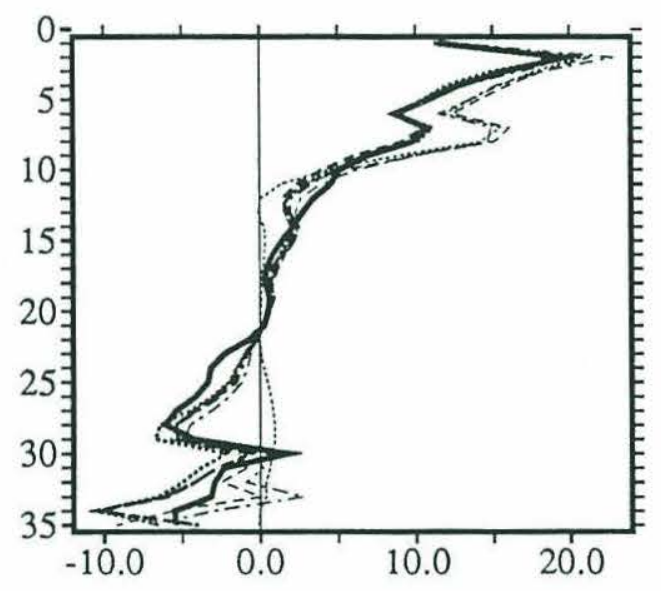

e.

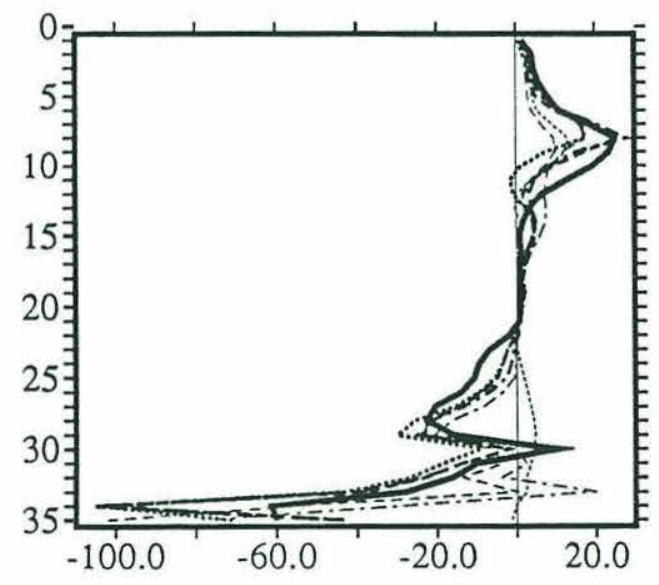

b.

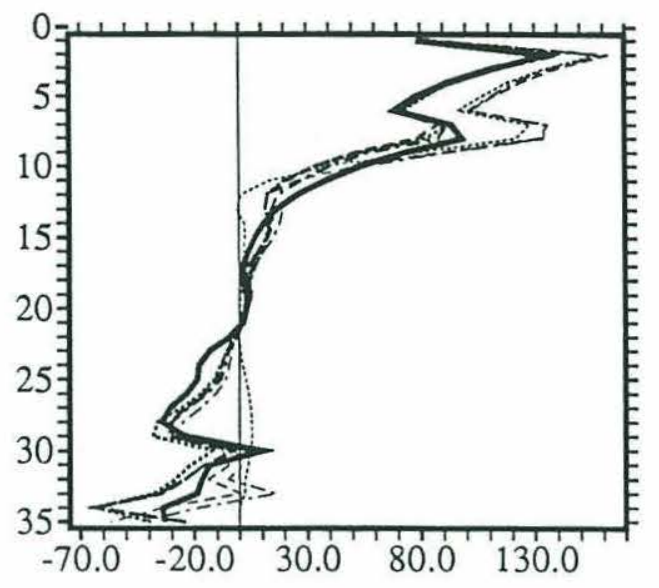

d.

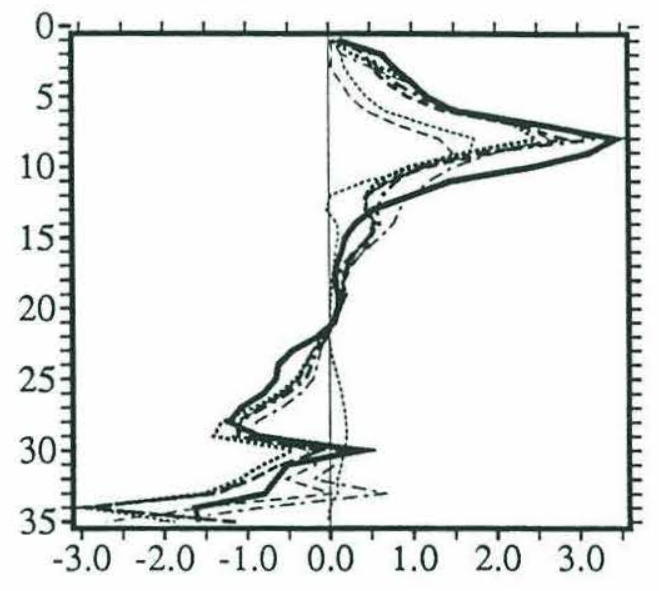

f.

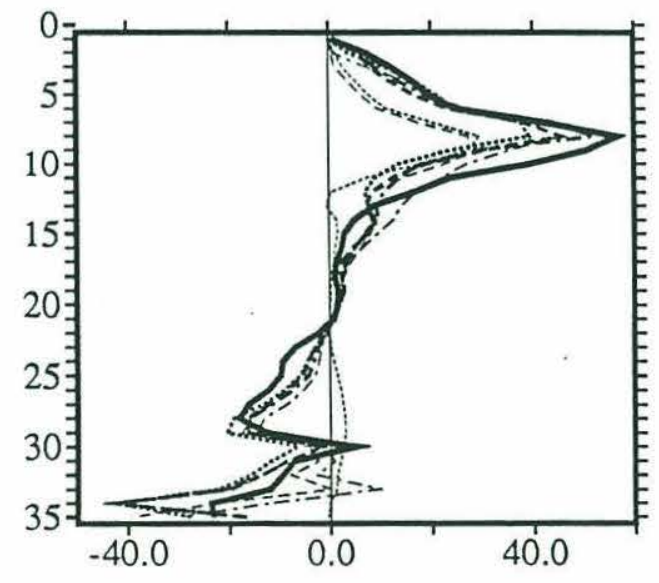

Figure 1-14. Property Fluxes vs Depth. The different curves are as in Figure 1-12.

(a) heat flux ( $\left.{ }^{\circ} \mathrm{C} \cdot \mathrm{Sv}\right)(\mathrm{b})$ salt flux (PSU.Sv) (c) oxygen flux $(\mathrm{ml} / \mathrm{l} \cdot \mathrm{Sv}$ )

(d) phosphate flux $(\mu \mathrm{mol} / \mathrm{kg} \cdot \mathrm{Sv})$ (e) silicate flux $(\mu \mathrm{mol} / \mathrm{kg} \cdot \mathrm{Sv})$ (f) nitrate flux $(\mu \mathrm{mol} / \mathrm{kg} \cdot \mathrm{Sv})$ 
a.

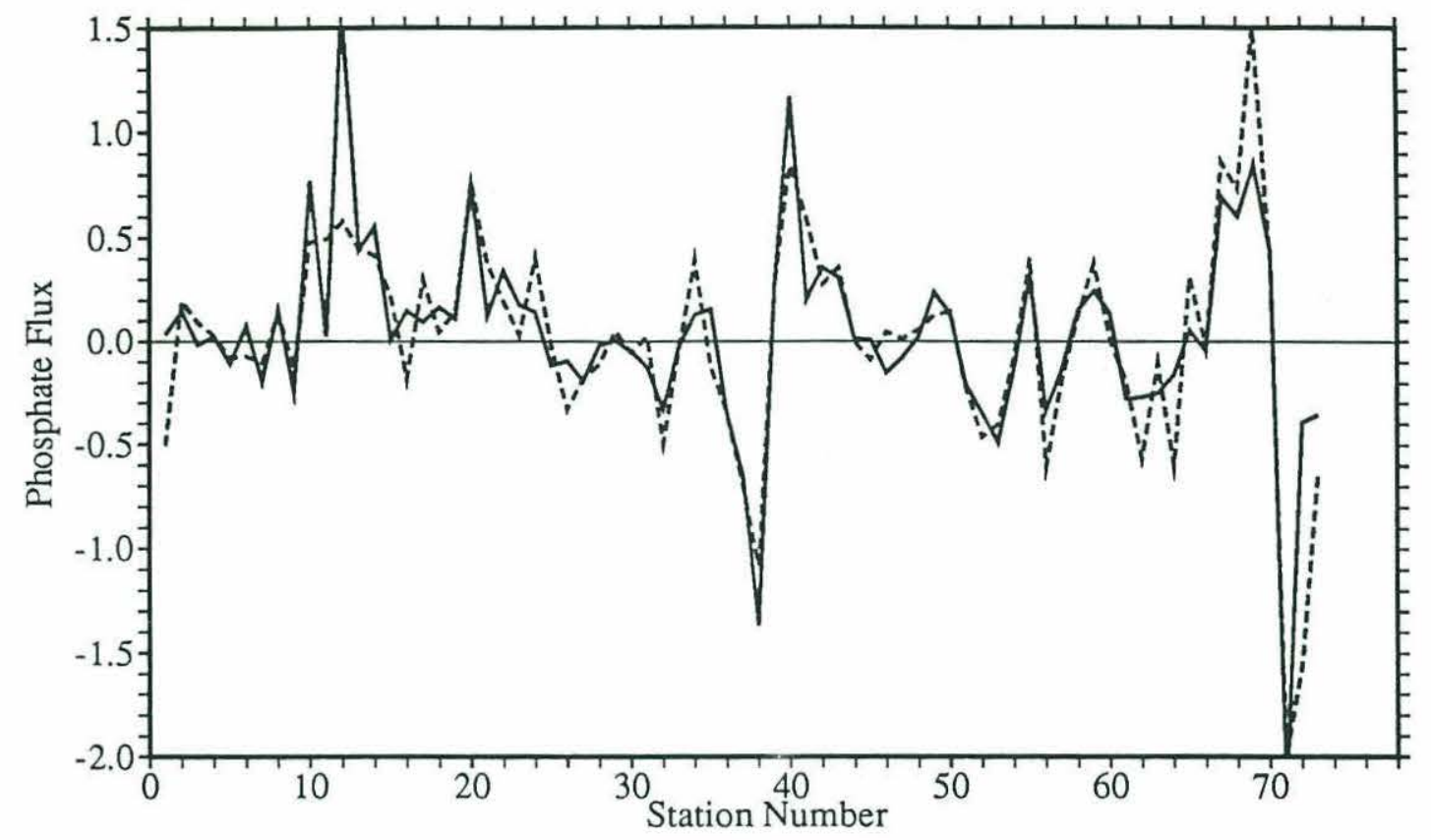

b.

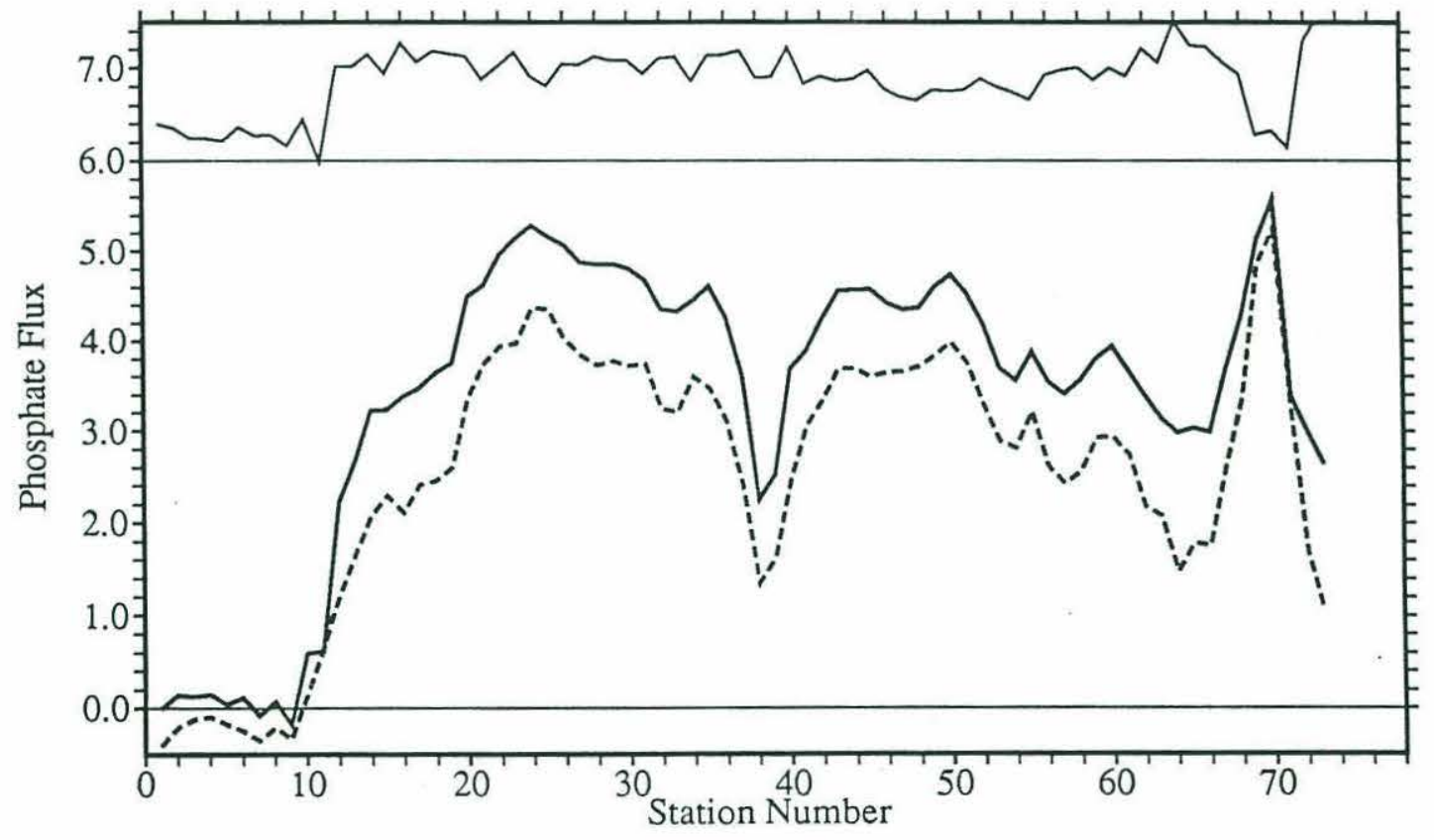

Figure 1-15. Phosphate flux ( $\mu \mathrm{mol} / \mathrm{kg} \cdot \mathrm{Sv})$ at $600 \mathrm{~m}$, as in Figure 1-12.

(a) Phosphate flux between each station pair.

(b) Cumulative phosphate flux from the northern end. 
From dynamic calculations, we can compute the property fluxes just as we did for mass flux. Figure 1-14 shows net zonal flux of heat $\left({ }^{\circ} \mathrm{C} \cdot \mathrm{Sv}\right)$, salt (PSU.Sv), oxygen $(\mathrm{ml} / \mathrm{l} \cdot \mathrm{Sv})$, phosphate, silicate and nitrate (all in $\mu \mathrm{mol} / \mathrm{kg} \cdot \mathrm{Sv})$. As the units show, these are not the usual ones, but since the purpose of this exercise is to get an idea of how good the modal reconstruction compares with the original data, this form will suffice. All of these fluxes show agreement similar to what we saw with mass flux, except possibly for some of the nutrient fluxes at about $600 \mathrm{~m}$ (depth 10). Figure 1-15 plots the flux difference for phosphate at $600 \mathrm{~m}$. The discrepancy occurs due to the difference at some isolated station pairs (e.g. between stations 10 and 11 which is one of the station pairs in the Gulf Stream) and does not result from a systematic bias.

Judging from these flux comparisons, rank 4 appears to be the minimum rank that would account for the dominant large scale hydrographic structures of this section to within what we may consider noise (Section 4.2).

\section{Discussion}

\subsection{Correlation between Different Properties}

As pointed out in Figure 1-6, there is a high degree of similarity among the elements of $\underline{v}_{i}$. The first modes of temperature and salinity appear extremely similar to those of the three nutrients, except in sign. Similarly, the three nutrients appear identical among themselves for mode 2 . The elements of $\underline{v}_{i}$ can be thought of as the expansion coefficients of a hydrographic station in the vertical modes $\underline{u}_{i}$, since they can be written as,

$$
\underline{v}_{i}^{T}=\lambda_{i}^{-1} \underline{u}_{i}^{T} A .
$$

Similarity among them would indicate that the horizontal variation of one tracer is strongly correlated with another. If they were exactly the same, the modally reconstructed tracer variations would be identical, except for the mean and normalization 
we performed for nondimensionalization. Thus, as a measure of independence, we will examine the relations between the expansion coefficients.

It is worth pointing out what these property correlations mean with relation to property-property laws, such as a tight T-S relation on a T-S diagram. The expansion coefficients are indications of variations from the mean vertical profile. The fact that the coefficients are similar means that the two properties vary similarly at each depth. In property-property space, this would mean that a series of points will scatter in the same direction from their corresponding point on the mean profile, except for the different normalizations with depth. In other words, property correlations of this sort are indicators of presence of a preferred direction of scatter in property space. However, this direction is tuned to best describe the spatial variation of the properties. So close it may be, this direction is not necessarily parallel to the local axis of correlation on the property-property diagram. Examination of such property-property laws is presented in Section 6.3.

Let $\underline{t}$ and $\underline{s}$ be the horizontal expansion coefficients of, say, temperature and salinity, for the $i$ 'th vertical mode. As a simple measure of correlation, we can define a correlation coefficient,

$$
\gamma=\frac{\underline{t}^{T} \cdot \underline{s}}{\|\underline{t}\| \cdot\|\underline{s}\|} .
$$

If the elements of $\underline{t}$ had little to do with $\underline{s}$, the sum $\underline{t}^{T} \cdot \underline{s}$ would add up destructively and be small. It is noteworthy that the definition given by equation (1-11) is the same as the correlation coefficient between the $i$ 'th mode contribution of temperature and salinity over the entire section. That is, based on equations (1-4a) and (1-10), we can write,

$$
\gamma=\frac{\sum_{k l}{ }^{i} T_{k l}{ }^{i} S_{k l}}{\left\|{ }^{i} T\right\| \cdot\left\|^{i} S\right\|},
$$

where,

$$
\begin{aligned}
& { }^{i} T_{k l}=\lambda_{i}\left(\underline{u}_{i} \underline{t}^{T}\right)_{k l} \\
& { }^{i} S_{k l}=\lambda_{i}\left(\underline{u}_{i} \underline{s}^{T}\right)_{k l} .
\end{aligned}
$$


${ }^{i} T_{k l}$ and ${ }^{i} S_{k l}$ are the $i$ 'th mode contributions (like the individual terms in the expansion of equation 1-4a) at depth $k$ and station $l$ for temperature and salinity, respectively. ( $A_{k l}: k^{\prime}$ th row $l$ 'th column of matrix $A$.) Equation (1-12) is the usual form in which a correlation between property variations would be calculated. But what the modal expansion gives is a more concise description of these correlations. Equation (1-11) is based on vectors $(\underline{t}, \underline{s})$, while equation $(1-12)$ is based on matrices $\left({ }^{i} T,{ }^{i} S\right)$ whose row dimensions are the same as the vectors but column dimensions are the number of depths. To show the equivalence of equations (1-11) and (1-12), equation (1-12) can be expanded as follows. Using the orthonormality of $\underline{u}_{i}$, we evaluate the numerator of equation (1-12) as,

$$
\begin{aligned}
\sum_{k l}{ }^{i} T_{k l}{ }^{i} S_{k l} & =\sum_{k l} \lambda_{i}^{2}\left(u_{i, k} t_{l} u_{i, k} s_{l}\right) \\
& =\sum_{l} \lambda_{i}^{2}\left(\sum_{k} u_{i, k}^{2}\right) t_{l} s_{l} \\
& =\lambda_{i}^{2} \sum_{l} t_{l} s_{l}=\lambda_{i}^{2} \underline{t}^{T} \underline{s} .
\end{aligned}
$$

( $u_{i, k}$ is the $k$ th element of $\underline{u}_{i}$, and $t_{l}$ is the $l$ th element of $\left.\underline{t}.\right)$ The denominator is,

$$
\left\|^{i} T\right\|=\left(\lambda_{i}^{2} \sum_{k l} u_{i, k}^{2} t_{l}^{2}\right)^{1 / 2}=\lambda_{i}\|\underline{t}\| .
$$

Thus,

$$
\frac{\sum_{k l}{ }^{i} T_{k l}{ }^{i} S_{k l}}{\|\|^{i} T\|\cdot\|^{i} S \|}=\frac{\underline{t}^{T} \cdot \underline{s}}{\|\underline{t}\| \cdot\|\underline{s}\|} .
$$

Table 1-4 shows this correlation coefficient among the six variables for the first 4 modes. Here, we are reassured about the qualitative similarity seen in Figure 1-6. For example, mode 1 temperature expansion coefficients are highly correlated with salinity, and also with the three nutrients as well, but with an opposite sign. Oxygen on the other hand, is hardly correlated with any of the other variables in this mode, which is due to the absence of cross-Gulf Stream contrast (Figure 1-6). For mode 2, oxygen becomes somewhat weakly correlated with the rest, while temperature and salinity 
a. Mode 1

\begin{tabular}{|l|c|c|c|c|c|c|}
\hline & $\mathrm{T}$ & $\mathrm{S}$ & $\mathrm{O}$ & $\mathrm{P}$ & $\mathrm{Si}$ & $\mathrm{N}$ \\
\hline $\mathrm{T}$ & 1.00 & 0.90 & -0.35 & -0.89 & -0.77 & -0.88 \\
$\mathrm{~S}$ & 0.90 & 1.00 & -0.12 & -0.96 & -0.92 & -0.92 \\
$\mathrm{O}$ & -0.35 & -0.12 & 1.00 & -0.00 & -0.16 & -0.02 \\
$\mathrm{P}$ & -0.89 & -0.96 & -0.00 & 1.00 & 0.95 & 0.98 \\
$\mathrm{Si}$ & -0.77 & -0.92 & -0.16 & 0.95 & 1.00 & 0.93 \\
$\mathrm{~N}$ & -0.88 & -0.92 & -0.02 & 0.98 & 0.93 & 1.00 \\
\hline
\end{tabular}

b. Mode 2

\begin{tabular}{|c|c|c|c|c|c|c|}
\hline & $\mathrm{T}$ & $\mathrm{S}$ & $\mathrm{O}$ & $\mathrm{P}$ & $\mathrm{Si}$ & $\mathrm{N}$ \\
\hline $\mathrm{T}$ & 1.00 & 0.91 & -0.55 & 0.11 & -0.07 & 0.15 \\
$\mathrm{~S}$ & 0.91 & 1.00 & -0.59 & 0.20 & 0.05 & 0.24 \\
$\mathrm{O}$ & -0.55 & -0.59 & 1.00 & -0.79 & -0.71 & -0.81 \\
$\mathrm{P}$ & 0.11 & 0.20 & -0.79 & 1.00 & 0.93 & 0.95 \\
$\mathrm{Si}$ & -0.07 & 0.05 & -0.71 & 0.93 & 1.00 & 0.94 \\
$\mathrm{~N}$ & 0.15 & 0.24 & -0.81 & 0.95 & 0.94 & 1.00 \\
\hline
\end{tabular}

c. Mode 3

\begin{tabular}{|l|c|c|c|c|c|c|}
\hline & $\mathrm{T}$ & $\mathrm{S}$ & $\mathrm{O}$ & $\mathrm{P}$ & $\mathrm{Si}$ & $\mathrm{N}$ \\
\hline $\mathrm{T}$ & 1.00 & 0.50 & 0.21 & -0.68 & -0.17 & -0.53 \\
$\mathrm{~S}$ & 0.50 & 1.00 & -0.42 & -0.54 & -0.15 & -0.16 \\
$\mathrm{O}$ & 0.21 & -0.42 & 1.00 & -0.27 & -0.44 & -0.57 \\
$\mathrm{P}$ & -0.68 & -0.54 & -0.27 & 1.00 & 0.71 & 0.87 \\
$\mathrm{Si}$ & -0.17 & -0.15 & -0.44 & 0.71 & 1.00 & 0.84 \\
$\mathrm{~N}$ & -0.53 & -0.16 & -0.57 & 0.87 & 0.84 & 1.00 \\
\hline
\end{tabular}

Table 1-4. Modal Correlation Coefficient

Modal correlation coefficients for the first four modes are shown. 
d. Mode 4

\begin{tabular}{|l|c|c|c|c|c|c|}
\hline & $\mathrm{T}$ & $\mathrm{S}$ & $\mathrm{O}$ & $\mathrm{P}$ & $\mathrm{Si}$ & $\mathrm{N}$ \\
\hline $\mathrm{T}$ & 1.00 & 0.82 & -0.08 & -0.59 & -0.68 & -0.59 \\
$\mathrm{~S}$ & 0.82 & 1.00 & 0.04 & -0.67 & -0.65 & -0.63 \\
$\mathrm{O}$ & -0.08 & 0.04 & 1.00 & -0.55 & -0.50 & -0.55 \\
$\mathrm{P}$ & -0.59 & -0.67 & -0.55 & 1.00 & 0.91 & 0.93 \\
$\mathrm{Si}$ & -0.68 & -0.65 & -0.50 & 0.91 & 1.00 & 0.94 \\
$\mathrm{~N}$ & -0.59 & -0.63 & -0.55 & 0.93 & 0.94 & 1.00 \\
\hline
\end{tabular}

Table 1-4. (continued)

\begin{tabular}{|l|c|c|c|c|c|c|}
\hline & $\mathrm{T}$ & $\mathrm{S}$ & $\mathrm{O}$ & $\mathrm{P}$ & $\mathrm{Si}$ & $\mathrm{N}$ \\
\hline $\mathrm{T}$ & 1.00 & 0.86 & -0.30 & -0.52 & -0.44 & -0.46 \\
$\mathrm{~S}$ & 0.86 & 1.00 & -0.28 & -0.57 & -0.47 & -0.45 \\
$\mathrm{O}$ & -0.30 & -0.28 & 1.00 & -0.45 & -0.51 & -0.54 \\
$\mathrm{P}$ & -0.52 & -0.57 & -0.45 & 1.00 & 0.91 & 0.95 \\
$\mathrm{Si}$ & -0.44 & -0.47 & -0.51 & 0.91 & 1.00 & 0.93 \\
$\mathrm{~N}$ & -0.46 & -0.45 & -0.54 & 0.95 & 0.93 & 1.00 \\
\hline
\end{tabular}

Table 1-5. Correlation Coefficient at rank 4

Modal correlation coefficients for the sum of the first four modes are shown. 
become uncorrelated with the three nutrients. Correlation between temperature and salinity becomes weak for mode 3 , due to the difference between the variations of the two towards the southern end of the section and the extreme northern end (Figure 1-6). The correlation among the three nutrients remains high for all 4 modes.

These correlations are for each separate mode's contribution. We can calculate the same correlation coefficient for the sum of modal contributions according to equation (1-12) where $T$ is now a sum of all modal contributions,

$$
T=\lambda_{1} \underline{u}_{1} \underline{t}_{1}^{T}+\lambda_{2} \underline{u}_{2} \underline{t}_{2}^{T}+\cdots+\lambda_{k} \underline{u}_{k} \underline{t}_{k}^{T} .
$$

Then,

$$
\begin{aligned}
\sum_{i j} T_{i j} S_{i j} & =\sum_{i j}\left(\lambda_{1} u_{1, i} t_{1, j}+\cdots+\lambda_{k} u_{k, i} t_{k, j}\right)\left(\lambda_{1} u_{1, i} s_{1, j}+\cdots+\lambda_{k} u_{1, i} s_{k, j}\right) \\
& =\sum_{j}\left(\lambda_{1}^{2} t_{1, j} s_{1, j}+\cdots \lambda_{k}^{2} t_{k, j} s_{k, j}\right) \\
& =\sum_{l=1}^{k} \lambda_{l}^{2} \underline{t}_{l}^{T} \underline{s}_{l},
\end{aligned}
$$

and,

$$
\left\|T_{i j}\right\|=\left\|\left(\lambda_{1} \underline{t}_{1}, \lambda_{2} \underline{t}_{2}, \ldots, \lambda_{k} \underline{t}_{k}\right)\right\| .
$$

This overall correlation using equations (1-12), (1-14) and (1-15) is given in Table 1-5, for the sum of the first 4 modes. In essence, this is an average over the correlations in Table 1-4. The overall correlation indicates that there are essentially two largely correlated groups. One is temperature and salinity and the other is the three nutrients (phosphate, silicate, nitrate). Oxygen remains relatively weakly correlated with other variables.

The fact that there are strong correlations between properties indicates yet another redundancy among the data. Correlation means that if we knew the variation of one or some combination of variables, we could estimate another with good accuracy. To 
eliminate the redundancy most efficiently, we can identify the degrees of freedom among the modal expansion coefficients themselves. We will use the term property modes for these degrees of freedom as opposed to spatial modes derived previously.

Consider the following matrix,

$$
D_{6 \times(80 \times 4)}=\left(\begin{array}{cccc}
\lambda_{1} \underline{t}_{1}^{T} & \lambda_{2} \underline{t}_{2}^{T} & \lambda_{3} \underline{t}_{3}^{T} & \lambda_{4} \underline{t}_{4}^{T} \\
\lambda_{1} \underline{s}_{1}^{T} & \lambda_{2} \underline{s}_{2}^{T} & \lambda_{3} \underline{s}_{3}^{T} & \lambda_{4} \underline{s}_{4}^{T} \\
\lambda_{1} \underline{\underline{O}}_{1}^{T} & \lambda_{2} \underline{\underline{o}}_{2}^{T} & \lambda_{3} \underline{\underline{o}}_{3}^{T} & \lambda_{4} \underline{\underline{O}}_{4}^{T} \\
\lambda_{1} \underline{p}_{1}^{T} & \lambda_{2} \underline{p}_{2}^{T} & \lambda_{3} \underline{p}_{3}^{T} & \lambda_{4} \underline{p}_{4}^{T} \\
\lambda_{1} \underline{s}_{1}^{T} & \lambda_{2} \underline{s}_{2}^{T} & \lambda_{3} \underline{s}_{3}^{T} & \lambda_{4} \underline{s}_{4}^{T} \\
\lambda_{1} \underline{n}_{1}^{T} & \lambda_{2} \underline{n}_{2}^{T} & \lambda_{3} \underline{n}_{3}^{T} & \lambda_{4} \underline{n}_{4}^{T}
\end{array}\right),
$$

Each row corresponds to one of the six variables, and each column is a modal expansion coefficient for a particular mode (spatial) at a particular station (e.g. $\underline{t}_{i}$ is the expansion coefficient of temperature for mode $i$ ). We include the eigenvalues of the spatial modes to explicitly state the significance of each mode (Section 4.1). The result of an SVD of $D$ is summarized in Table 1-6.

The singular values of matrix $D$ provide a measure (Section 4.1) of how many degrees of freedom there are among the horizontal variations of the six variables (viz. the expansion coefficients of the six properties). According to Table 1-6, there are two dominant modes which together will account for $93 \%$ of the entire variance in $D$. The corresponding column singular vectors define linear combinations of the six original variables. These combinations can be thought of as new variables whose horizontal distributions are "most common" (in the sense described in Section 4.1) among the six original variables and are mutually independent. The first two modes given in Table 1-6 define the following two variables;

$$
\begin{aligned}
& \alpha=0.38 \cdot T+0.41 \cdot S+0.11 \cdot O-0.49 \cdot P-0.50 \cdot S i-0.44 \cdot N \\
& \beta=-0.51 \cdot T-0.53 \cdot S+0.53 \cdot O-0.19 \cdot P-0.28 \cdot S i-0.26 \cdot N .
\end{aligned}
$$




\begin{tabular}{|c|c|c|c|c|c|c|}
\hline & 1 & 2 & 3 & 4 & 5 & 6 \\
\hline$e$ & 130. & 83.0 & 26.0 & 23.0 & 20.0 & 12.0 \\
$\%$ & 66.5 & 26.6 & 2.68 & 2.03 & 1.60 & 0.52 \\
\hline \multirow{4}{*}{$\underline{u}$} & 0.38 & -0.51 & 0.71 & -0.19 & 0.17 & 0.15 \\
& 0.41 & -0.53 & -0.64 & -0.32 & 0.04 & -0.21 \\
& 0.11 & 0.53 & 0.05 & -0.82 & 0.20 & 0.02 \\
& -0.49 & -0.19 & 0.09 & -0.04 & 0.62 & -0.58 \\
& -0.50 & -0.28 & 0.14 & -0.42 & -0.68 & -0.16 \\
& -0.44 & -0.26 & -0.22 & -0.14 & 0.30 & 0.76 \\
\hline
\end{tabular}

Table 1-6. Result of SVD of $D$.

The first row entry lists the singular values $(e)$ of $D$ with the percentage variance of each property mode. The second entry is the column singular vectors $(\underline{u})$ of $D$.

a.

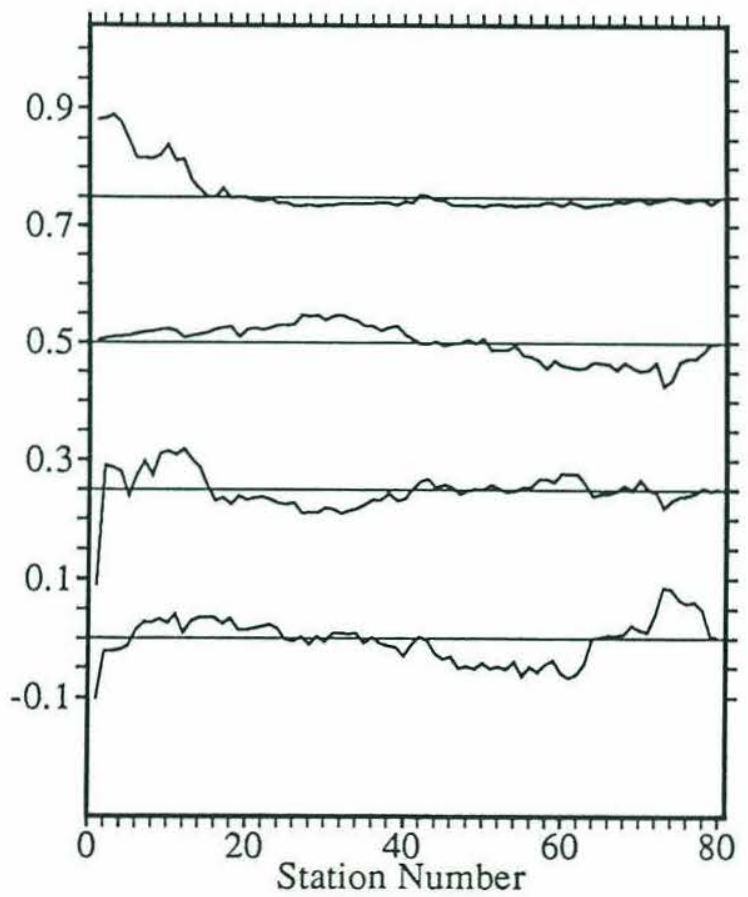

b.

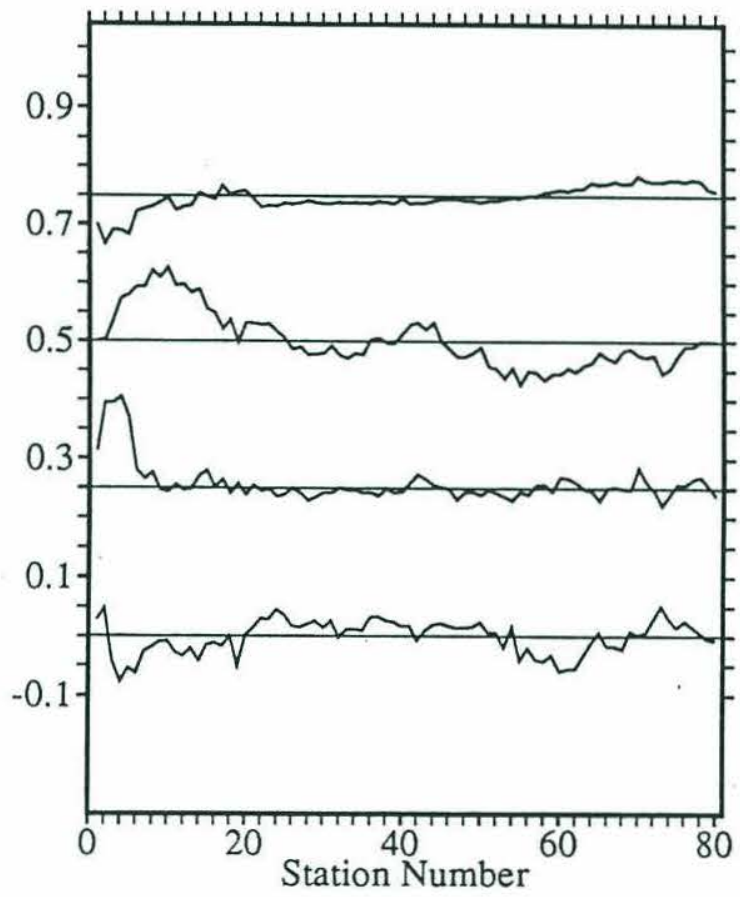

Figure 1-16. Expansion Coefficients of the Property Modes.

(a) first property mode (b) second property mode

The top most curve in each figure corresponds to the first spatial mode, with higher spatial mode results each offset downward. The extreme left is the northern end of the section. 
The values of $\alpha$ and $\beta$ for the first four spatial modes are shown in Figure $1-16$. We immediately notice some similarities of these expansion coefficients with the expansion coefficients of the six original variables (Figure 1-6). From this comparison and the coefficients defining $\alpha$ and $\beta$ (equation 1-17), we see that $\alpha$ mostly describes the similarities between the horizontal distributions of temperature-salinity with phosphatesilicate-nitrate. (These two groups are negatively correlated as seen in Tables 1-4 and 1-5.) $\beta$ describes mainly the differences of temperature-salinity and oxygen (and also with phosphate-silicate-nitrate) which are not accounted for by $\alpha$.

\subsection{Dynamic Height Perturbations}

Each modal contribution can be viewed as a perturbation to the mean vertical profile of temperature, salinity, etc. Similarly, we can ask what each mode does to the mean dynamic height. Consider the dynamic height perturbation to the mean due to an RMS perturbation of temperature and salinity. The RMS temperature perturbation of the $i$ 'th mode is,

$$
\underline{t}_{i}^{\prime}=\lambda_{i} \underline{u}_{i} \sqrt{<v_{i}^{2}>} \text {. }
$$

$\sqrt{\left\langle v_{i}^{2}\right\rangle}$ is the RMS value of temperature expansion coefficient for the $i$ 'th mode (elements of $\underline{v}_{i}$ ). As each mode explains a data variance nearly equally for different variables (Table 1-3), this expected magnitude is uniform among the variables according to equation $(1-4 \mathrm{~b})$. Then, since $\underline{v}_{i}$ is unit length by definition,

$$
\sqrt{<v_{i}^{2}>}=\frac{1}{\sqrt{6 n}}
$$

$n$ is the number of stations used in this hydrographic section ( $6 n$, which is the column dimension of $A$, because there are six variables). In dimensional form equation (1-18) is,

$$
\underline{t}_{i}^{\prime}=\frac{\lambda_{i}}{\sqrt{6 n}} W_{T} \underline{u}_{i}
$$


where $W_{T}$ is a diagonal matrix which elements $\left(\sigma_{T, j}\right.$ for depth $\left.j\right)$ are the depth dependent normalization factors $\left(\sigma_{i}\right.$ in equation 1-6) for temperature. Similarly, the $i$ 'th mode RMS salinity contribution is,

$$
\underline{s}_{i}^{\prime *}=\frac{\lambda_{i}}{\sqrt{6 n}} W_{S} \underline{u}_{i} .
$$

As salinity is positively correlated with temperature (Table 1-4), the corresponding density perturbation of this mode is,

$$
\underline{\sigma}_{i \pm}^{\prime *}=\left|\underline{\sigma}(\underline{\bar{t}}, \underline{\bar{s}})-\underline{\sigma}\left(\underline{\bar{t}} \pm \underline{t}_{i}^{\prime *}, \underline{\bar{s}} \pm \underline{s}_{i}^{\prime *}\right)\right| .
$$

( $\underline{\bar{t}}$ is mean temperature profile.) The dynamic height differences between $\underline{\sigma}\left(\underline{\underline{t}} \pm \underline{t}_{i}^{\prime}{ }^{*}, \underline{\bar{s}} \pm \underline{s}_{i}^{\prime}{ }^{*}\right)$ and $\underline{\sigma}(\underline{\underline{t}}, \underline{s})$ are shown in Figure 1-17. The curves correspond to the first 4 modes, all referenced at $6000 \mathrm{~m}$ (depth 35 ).

Despite the fact that the equation of state is a nonlinear function of temperature and salinity, the dynamic height differences are highly symmetric between $\underline{\sigma}_{+}^{\prime *}$ and $\underline{\sigma}_{-}^{\prime *}$. This indicates that each mode's contribution to dynamic height is nearly linear. So, by using the values of the modal expansion coefficients, we might expect to calculate dynamic height through a linear combination of modal contributions without actually calculating temperature, etc., or using the equation of state. Figure 1-18 is such a comparison. These are the dynamic height differences from the mean temperaturesalinity profile at the surface referenced at $2000 \mathrm{~m}$. The solid curve is the original data. The dashed curve is based on temperature and salinity of the modal reconstruction at rank 4. The dotted curve is the same dynamic height when constructed from modal perturbations calculated as follows:

Let the dynamic height perturbation associated with the RMS temperature perturbation of mode $i$ be written as ${ }^{T} \underline{h}_{i}$,

$$
{ }^{T} \underline{h}_{i}=\frac{1}{2}\left\{\left(\underline{H}\left(\underline{\bar{t}}+\underline{t}_{i}^{\prime *}, \underline{\bar{s}}\right)-\underline{H}(\underline{\bar{t}}, \underline{\bar{s}})\right)-\left(\underline{H}\left(\underline{\bar{t}}-\underline{t}_{i}^{\prime}, \underline{\bar{s}}\right)-\underline{H}(\underline{\bar{t}}, \underline{\bar{s}})\right)\right\}
$$




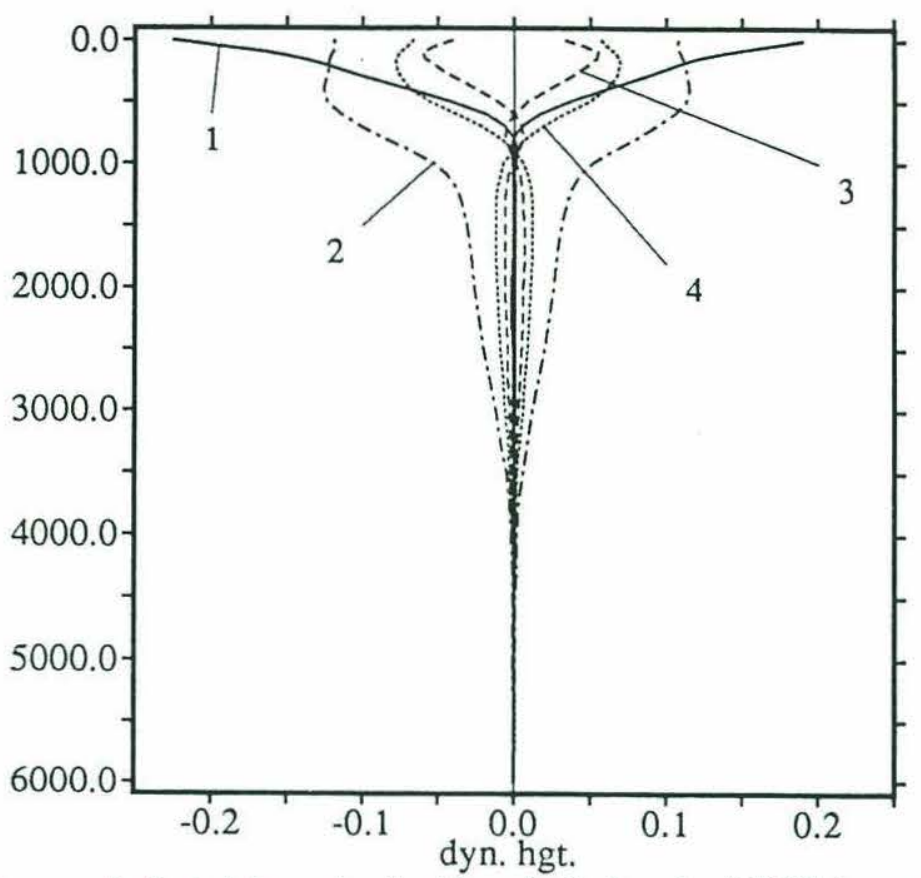

Figure 1-17. Dynamic height perturbation (m) due to RMS temperature and salinity perturbations. Solid curve is mode 1 , chain-dashed curve is mode 2, dash is mode 3 and dot is mode 4 . There are 2 curves for each mode, corresponding to the 2 signs in equation (1-21). All dynamic height perturbations are referenced to $6000 \mathrm{~m}$.

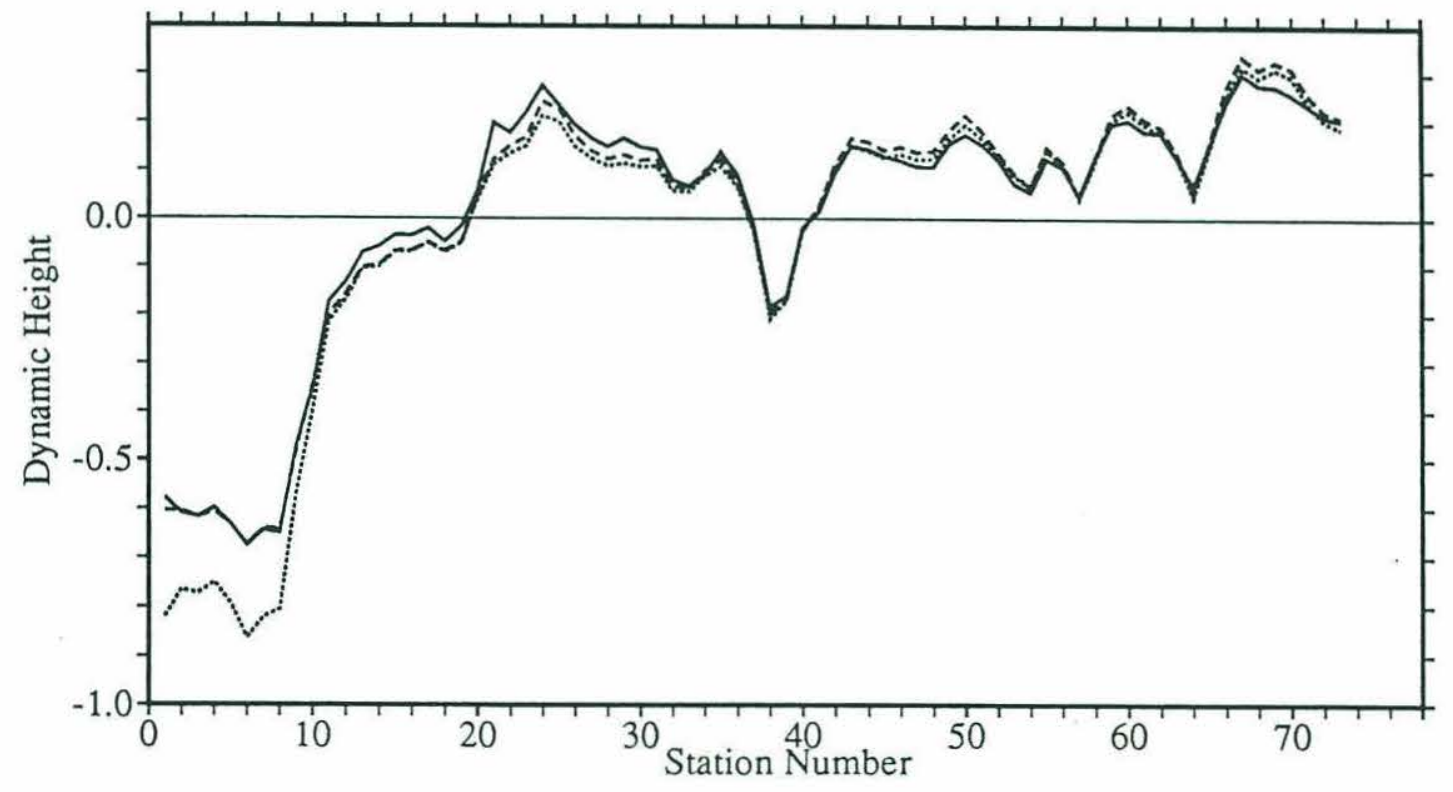

Figure 1-18. Dynamic height difference $(\mathrm{m})$ from the mean temperature and salinity profile at the surface, referenced at $2000 \mathrm{~m}$. Solid curve is the data. Dashed curve is based on temperature and salinity reconstructed with 4 modes. The dotted curve is from modal perturbations of dynamic height as in equation (1-24). 
$\underline{H}(\underline{\underline{t}}, \underline{s})$ is the dynamic height profile associated with a temperature and salinity profile of $\underline{\bar{t}}$ and $\underline{\bar{s}}$. We can also define a salinity mode perturbation ${ }^{S} \underline{h}_{i}$ likewise. Any temperature and salinity profile $\underline{t}$ and $\underline{s}$ can be written as,

$$
\begin{aligned}
& \underline{t}=\underline{\bar{t}}+\sum_{i} \frac{v_{i}}{\sqrt{\left.<v_{i}^{2}\right\rangle}} \underline{t}_{i}^{\prime *} \\
& \underline{s}=\underline{\bar{s}}+\sum_{i} \frac{v_{i}}{\sqrt{\left.<v_{i}^{2}\right\rangle}} s_{i}^{\prime *},
\end{aligned}
$$

where $v_{i}$ in each expression is the appropriate elements in Figure 1-6. By linearly approximating temperature and salinity perturbations to dynamic height, we can write,

$$
\underline{H}(\underline{t}, \underline{s}) \approx \underline{H}(\underline{\bar{t}}, \underline{\bar{s}})+\sum_{i} \frac{v_{i}}{\sqrt{\left.<v_{i}^{2}\right\rangle}} \cdot{ }^{T} \underline{h}_{i}+\sum_{i} \frac{v_{i}}{\sqrt{<v_{i}^{2}>}} \cdot{ }^{S} \underline{h}_{i} .
$$

The summation up to the fourth mode is shown as the dotted curve in Figure 1-18.

In the interior, the differences in Figure 1-18 are no larger than the short scale variations and there is even agreement across the cold core ring. However, the modal perturbation scheme (equation 1-24) is biased north of the Gulf Stream. This is the region where mode 1's coefficients become large, to cause a non-negligible contribution from the nonlinearity of the equation of state. One way of fixing this may be to expand $\underline{H}$ around a climatological mean of $\underline{t}$ and $\underline{s}$ rather than a zonal average. As the perturbations about the climatological mean can be expected to be smaller than the difference from the zonal average, the linear approximation, such as equation (1-24), would hold better.

\section{$6.3 T-S$ relations, etc.}

Since the modes are tuned for the optimal description of the spatial distribution of properties, it is not readily apparent that the modal reconstruction should retain the same properties such as a tight T-S relation. Figure 1-19 is a comparison of various property correlations between the data and the modal reconstruction at rank 4 . The modal reconstruction has slightly more scatter than the original data, but does retain 
a.

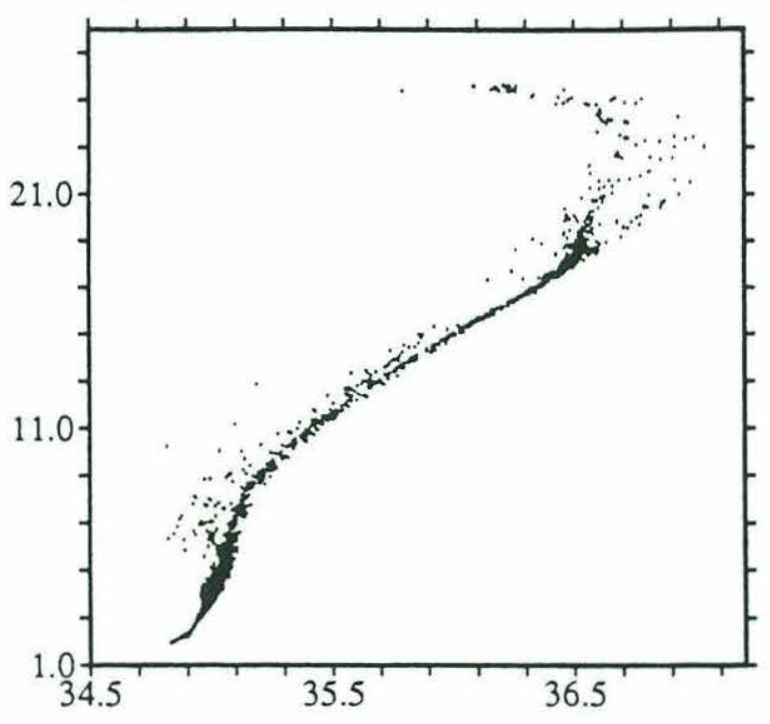

b.

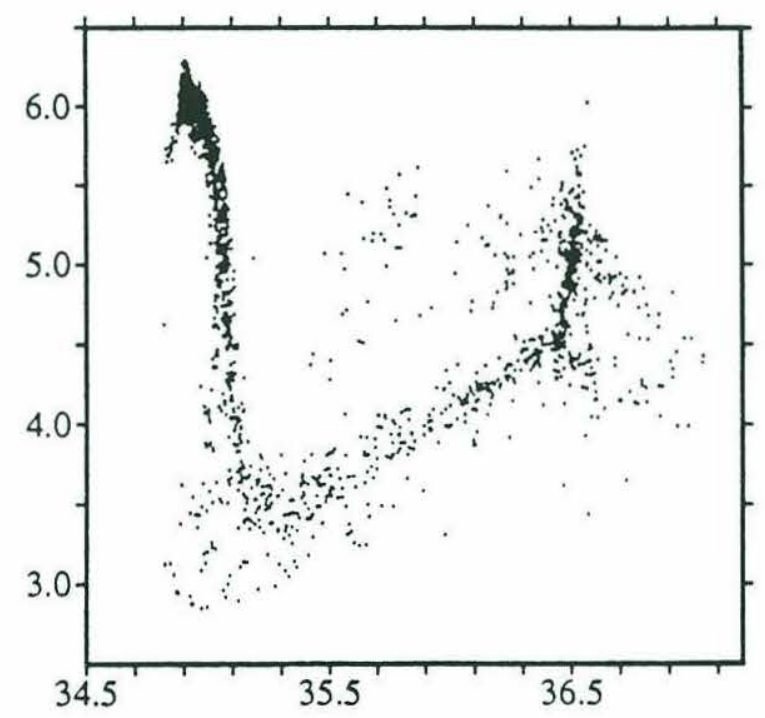

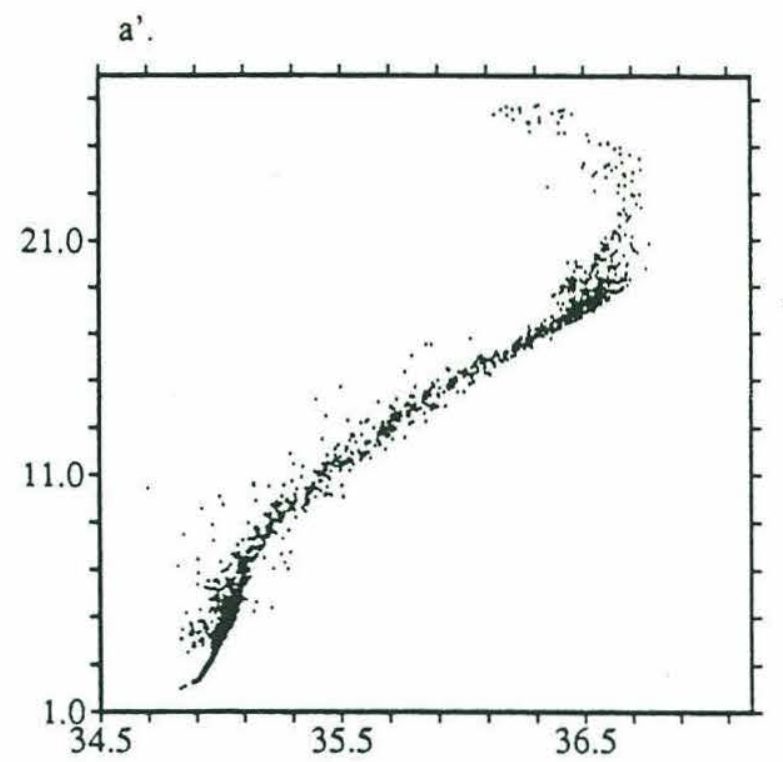

b'.

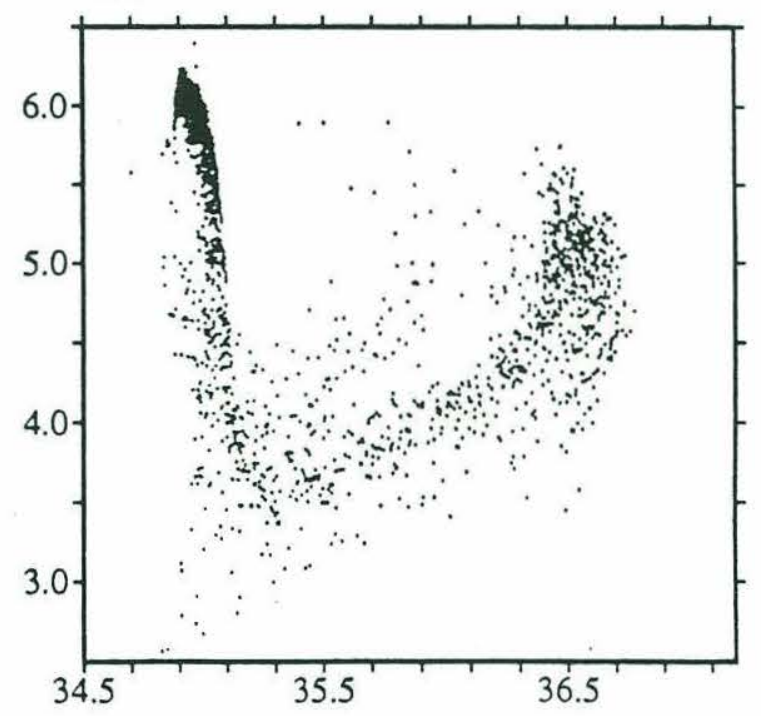

Figure 1-19. Scatter Diagram of Selected Properties.

(a) Temperature vs Salinity of data $\left(a^{\prime}\right)$ Temperature vs Salinity at rank 4

(b) Oxygen vs Salinity of data $\left(\mathrm{b}^{\prime}\right)$ Oxygen vs Salinity at rank 4 
c.

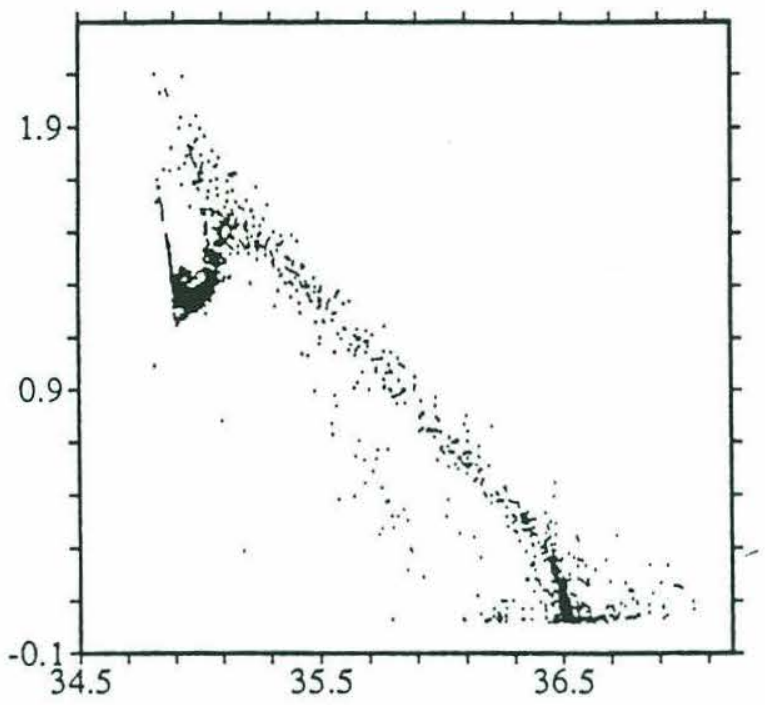

c'.

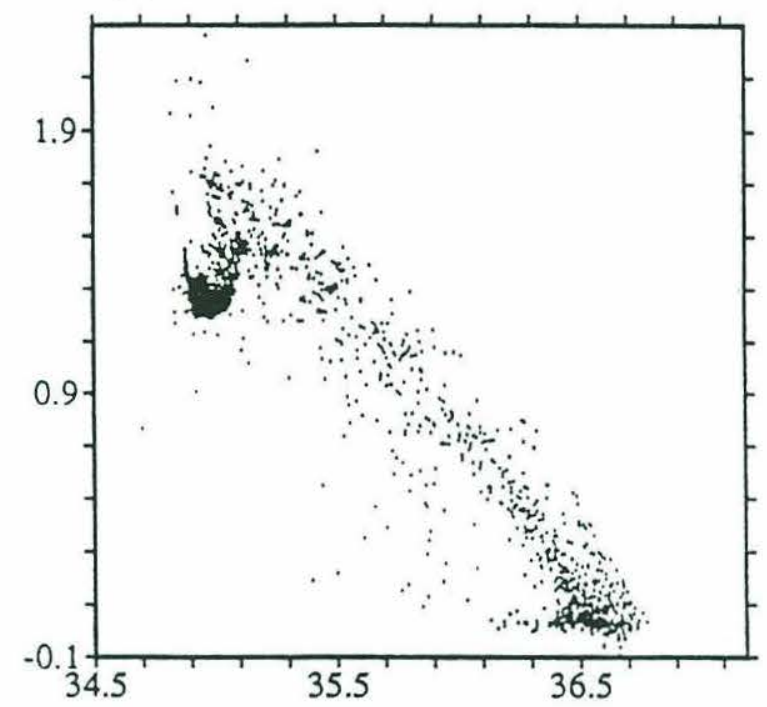

d.

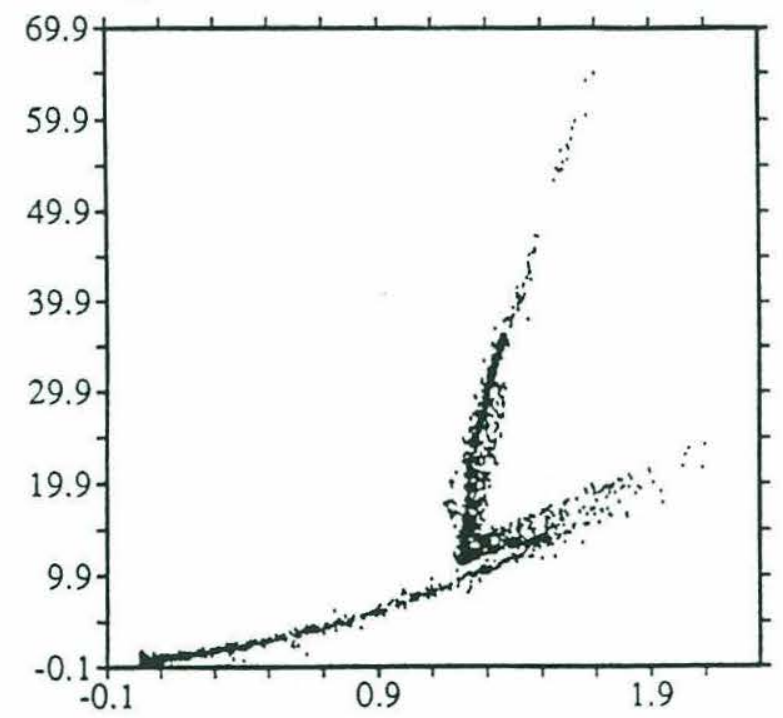

d'.

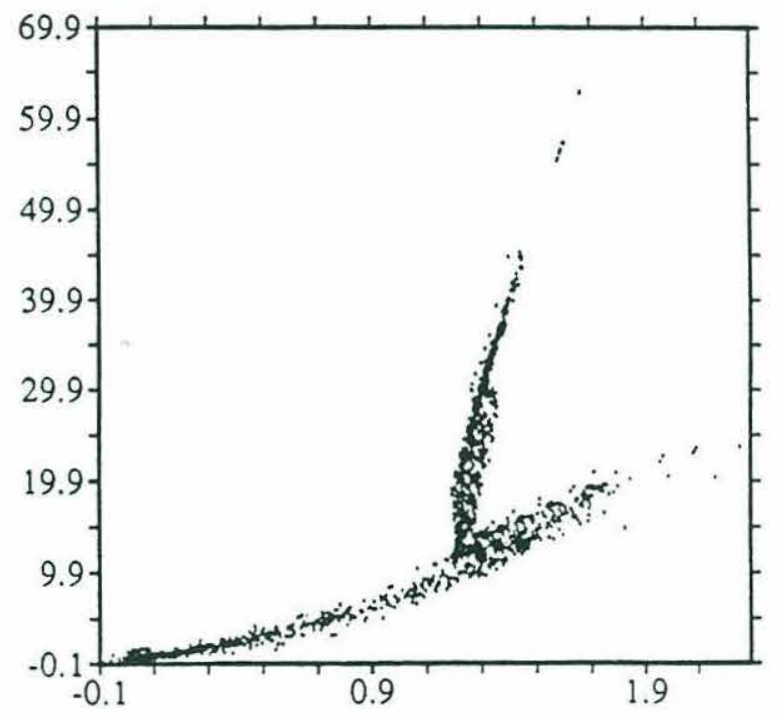

Figure 1-19. (continued)

(c) Phosphate vs Salinity of data $\left(c^{\prime}\right)$ Phosphate vs Salinity at rank 4

(d) Silicate vs Phosphate of data $\left(\mathrm{d}^{\prime}\right)$ Silicate vs Phosphate at rank 4 
the overall shape of the correlation. The scatter will decrease if more modes are added to the reconstruction. It is not clear, however, that a tight relation is the essential quality to look for. The null solution of the reconstruction, namely the mean vertical profile, has the tightest property correlation in the sense that the points will lie on a single curve in these plots close to the axis of the property-property law. However, the null solution does not contain any information on the spatial structure of the hydrographic section. Although the points lie on a single curve, the number of points is only the number of standard depths ( 35 in the present data set) and there are no points in between. Apparently, the scatter along the axis of the property law is part of the important qualities which reflect the hydrographic structure. The amount of scatter away from the mean curve is not uniform but has a coherent structure, and is also part of the nature of the property laws (e.g. Worthington, 1981).

Consider now what the modal perturbations mean in property space. In Figure 1-20, we show the RMS modal perturbations about the mean vertical profile on a T-S diagram for the first 4 modes. Each point corresponds to a different depth with the crosses being the mean vertical profile and the circles and squares, which are connected through the mean point by a straight line, are the modal perturbations. (Circle is positive vertical mode perturbation while square is negative.) RMS temperature and salinity perturbations are calculated as in equation (1-19) and (1-20). The high correlation between temperature and salinity expansion coefficients analyzed in Section 6.1 , means that the perturbations due to the modes will lie close to these lines or their extensions. This can be explained by considering how the temperature modal perturbations relate to those of salinity. The dimensional temperature and salinity perturbations of mode $i$ on depth $j$ are (at a particular station),

$$
\begin{aligned}
& t_{i, j}=\sigma_{T, j} \lambda_{i} u_{i, j} v_{t}, \\
& s_{i, j}=\sigma_{S, j} \lambda_{i} u_{i, j} v_{s} .
\end{aligned}
$$


A.

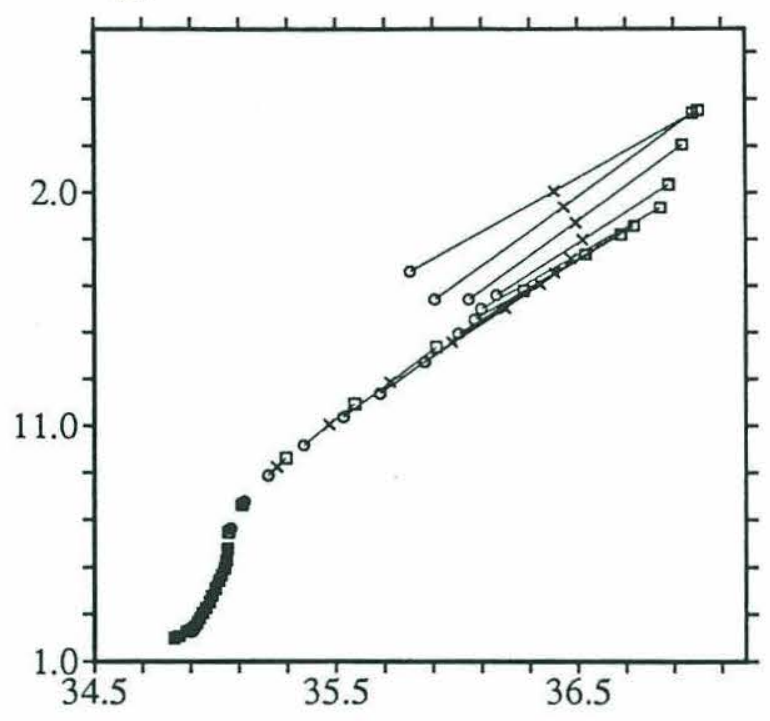

B.

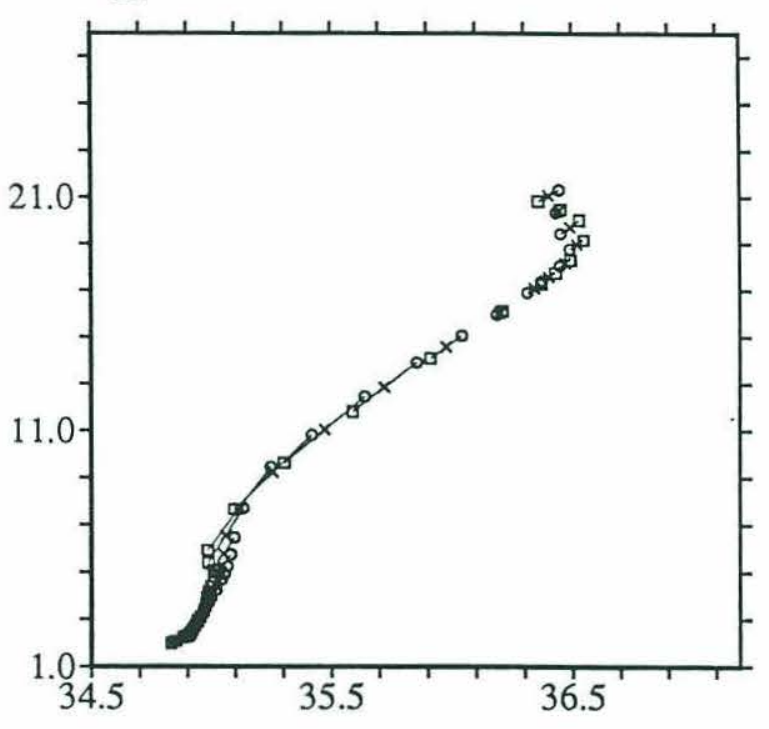

C.

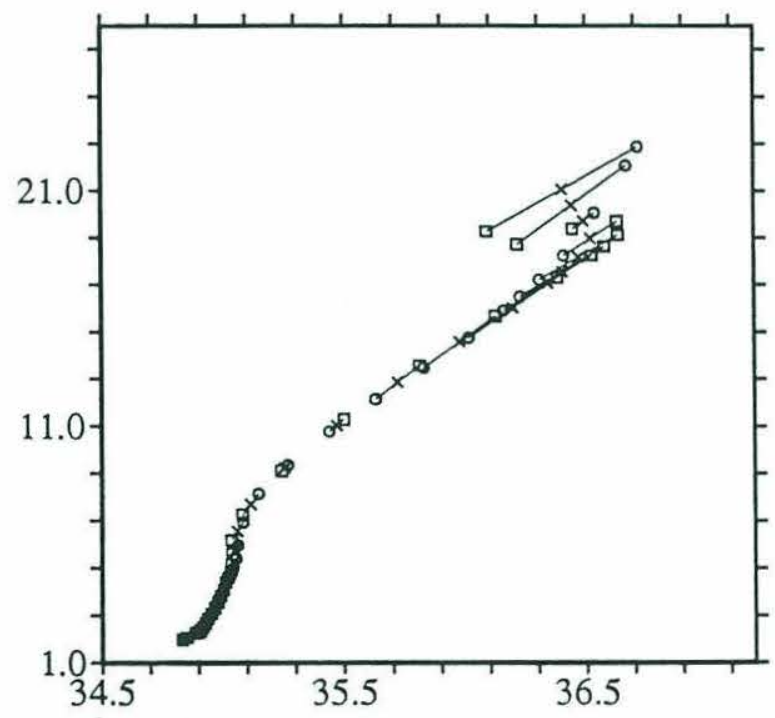

D.

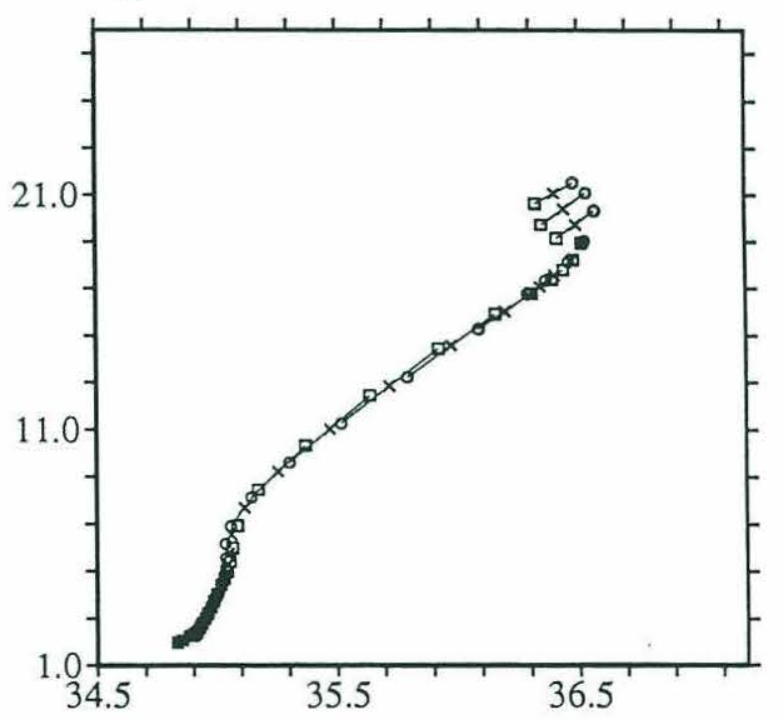

Figure 1-20. RMS modal perturbations on a T-S diagram.

The crosses correspond to the 35 points of the mean vertical profile. Circles and squares are the perturbed data point. Circle is a positive vertical mode perturbation, and the square is a negative one. The perturbed points are connected through their corresponding mean point by a straight line.

(a) Mode 1 (b) Mode 2 (c) Mode 3 (d) Mode 4 
Here, $\sigma_{T, j}$ and $\sigma_{S, j}$ are the normalization factors on depth $j$ of temperature and salinity, respectively. $v_{t}$ and $v_{s}$ are the modal expansion coefficients at this particular station. This perturbation has a slope in the T-S space defined by the ratio,

$$
\frac{\sigma_{T, j} v_{t}}{\sigma_{S, j} v_{s}} .
$$

If $v_{t}$ and $v_{s}$ are highly correlated, this ratio remains nearly constant on that depth throughout the section. The line defined by this slope is the preferred direction mentioned earlier in Section 6.1. Alternatively, an uncorrelated temperature and salinity vertical mode perturbation will cause the data to deviate from the mean in a direction away from these lines. The fact that the lines are not exactly parallel to each other at different depths reflects the different ratios in the normalization. The line segments about the thermocline $\left(8^{\circ} \mathrm{C} \sim 17^{\circ} \mathrm{C}\right)$ are nearly parallel to the axis of high correlation in all 4 modes. On the other hand, shallower and deeper perturbations are directed off the axis. This is consistent with the tendencies in the scatter plot of temperature-salinity data (Figure 1-19a). In the thermocline, the data mostly lie on the mean axis while shallower levels have a wider scatter about the main axis of correlation. Thus, the spatial modes do contain the information of the scatter in property space. The correlation in the expansion coefficient indicates the presence of a preferred direction of scatter and the size of the expansion coefficient indicates the magnitude of the scatter from the mean. The actual direction is given by the normalization factors, $\sigma_{i}$. However, the modal perturbation of each mode is directed from the mean vertical data points (the crosses) and may not be the best coordinate system to use when analyzing propertyproperty diagrams. The natural measures of property law are the distance from the axis of correlation and the density of that scatter; that is, how many data points are within a particular area of the diagram. Using one of the properties as the vertical coordinate might be a better coordinate system to describe the property law. (In turn, 
however, such descriptions will not be optimal in describing the spatial distribution of properties.)

In summary, although the spatial mode description is not the same as the real property laws, the modal reconstruction does retain the overall shape of the property correlations.

\section{Summary}

We have demonstrated the feasibility of a modal analysis of hydrographic data. The Endeavor cruise 129 along $64^{\circ} \mathrm{W}$ is used as a test data set. The analysis is based on variations of the properties from their mean vertical profile. The properties analyzed include: temperature, salinity, oxygen, phosphate, silicate and nitrate. An empirical orthogonal function analysis of these showed that most of the large scale coherence seen in all fields could be explained by the first 4 modes. This is a factor of almost 9 in the reduction of the number of degrees of freedom. Indeed, the virtue of the analysis lies in the simplicities of both the method and the result. The method allows us to identify the minimum degrees of freedom as an orthonormal set, which makes further analyses simple. The compactness of the result assures that the analyses of the data can be done with a minimum computational load.

The four modes reproduce most of the property flux of the original data set, and preserve the various property correlations as well. The modes are also able to resolve some of the strongest currents such as the Gulf Stream and a cold core ring. The similarities of spatial variations between separate variables are quantified by calculating their correlation coefficients. Analysis of the modal coefficients reveals that there are only two sets of independent data within these six variables. One is essentially temperature and salinity, while the other is the three nutrients and oxygen. This in turn would imply that these two sets contain quite different information regarding circulation, mixing, sources and sinks or anything which control their distribution. 
We have seen spatial coherence in the distribution of modal coefficients (elements of $\underline{v}_{i}$ ). By further analysis, we could relate the modal structures with hydrographic variations. We indicated some of these for mode 1 and 2, but we shall reserve further analysis until the next chapter, where we analyze the entire North Atlantic. There we will see the vertical modes have different shapes than the ones seen here. Apparently, what constitutes "dominant" depends on the geographic area of concern.

We have also found that the modal contribution to dynamic height is nearly linear. Dynamic height, too, can be expanded through the same set of modes. This observation, together with the other findings, prompts an interesting application. The spatial coherence of the elements of $\underline{v}_{i}$ means that we have some idea of the values of the modal coefficients as a function of geographic location. We also know the vertical profile of their dynamic height perturbations, which obviously are different from mode to mode. Then, if we have such data as the absolute dynamic height of the sea surface over an extensive area obtainable by satellite altimetry, we could perhaps make inferences of the three-dimensional structure and circulation underlying this observed surface topography. It would be done through estimation of the modal coefficients, by requiring the resulting field to obey certain kinematic and dynamic constraints but at the same time having a surface boundary condition on its height. Mathematically this will take a form of linear regression, which is operationally straightforward. The essence of this idea is that the modes provide means to relate structure at one location to another, be it horizontally or in depth, and the finding that each modal contribution, whether temperature or dynamic height, adds effectively linearly. 


\section{CHAPTER 2 \\ Hydrographic Modes of the North Atlantic Ocean}

\section{Introduction}

In Chapter 1, a small set of modes was shown to account for the essential hydrographic structures of a single north-south section. The analysis in Chapter 1 serves as a feasibility study of data reduction. In this present chapter, we expand the region of analysis to determine the minimum data that will describe the entire North Atlantic Ocean. Stations used in this chapter have a fairly good coverage of the ocean (Figure 2-1). The hydrographic data, described in more detail in Section 2, were all occupied between 1981 and 1985. The present study can be viewed as, in part, an analysis of the climatological state of the North Atlantic Ocean in the early 1980's.

This study encompasses more hydrographic variations than the single section along $64^{\circ} \mathrm{W}$ (Chapter 1), and whether a compact description exists is a nontrivial question. How modal variations resolve distribution of different water masses is an interesting question. Later in this chapter, we will combine the results from various hydrographic sections to map the modal structure over the whole ocean. This results in a model of the North Atlantic Ocean hydrography with minimum degrees of freedom. Analysis of the circulation of this model ocean will be the subject of the next chapter, Chapter 3 .

The subsequent discussion in the present chapter is made up of 9 parts. First, we describe in the next section the hydrographic data used in this study including sources, geography, dates of occupation, description of hydrographic structure by presenting contour plots of all available properties along the sections. Section 3 describes the normalization performed to the data prior to modal decomposition. Results of the modal decomposition are presented in Section 4. Sections 5 to 7 discuss what the modes resolve. Section 5 analyzes the residuals of the modal reconstructions to determine the number of significant modes. The spatial resolution of the modes is discussed in Section 


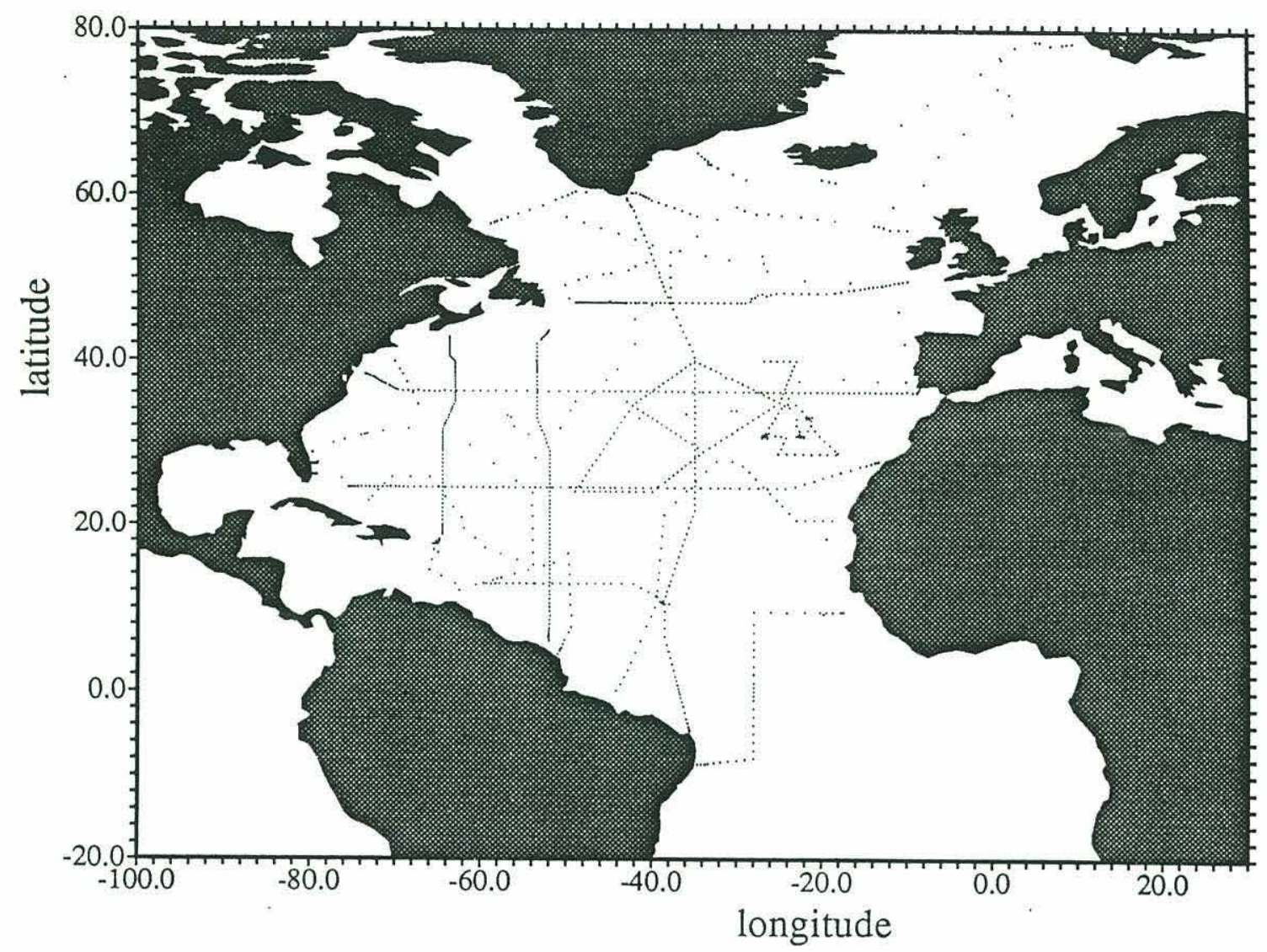

Figure 2-1. Station Locations

\begin{tabular}{|l|c|c|c|}
\hline Cruise & line & \# of stat. & Date \\
\hline Endeavor 129 & $64^{\circ} \mathrm{W}$ & 92 & $4 / 85$ \\
\hline Oceanus 133 leg 7 & $53^{\circ} \mathrm{W}$ & 94 & $5 / 83$ \\
\hline Knorr 104 & $35^{\circ} \mathrm{W}$ & 103 & $8 / 83$ \\
\hline Hudson 82 & $48^{\circ} \mathrm{N}$ & 78 & $4 / 82$ \\
\hline Atlantis II 109 leg 1 & $36^{\circ} \mathrm{N}$ & 101 & $6 / 81$ \\
\hline \multicolumn{1}{|c|}{$\operatorname{leg} 3$} & $24^{\circ} \mathrm{N}$ & 90 & $8 / 81$ \\
\hline & $26^{\circ} \mathrm{N}, 27^{\circ} \mathrm{N}$ & 22 & $9 / 81$ \\
\hline Oceanus 133 leg 2 & $13^{\circ} \mathrm{N}$ & 27 & $1 / 83$ \\
\hline TTO NAS & northern N. Atlantic & 250 & $7 / 81$ \\
\hline TTO TAS & tropical N. Atlantic & 109 & $1 / 83$ \\
\hline TOPOGULF & $\begin{array}{c}\text { mid-latitude } \\
\text { mid-Atl ridge }\end{array}$ & 115 & $8 / 83$ \\
& \multicolumn{3}{|l}{} \\
\hline
\end{tabular}

Table 2-1. Cruise Descriptions

After the name of each cruise or experiment, we list the nominal line or region of the hydrographic data, the number of stations, and the nominal date the sections were occupied. For further description of the data, refer to text in Section 2. 
6. We examine whether the circulation associated with the modal reconstruction is consistent with the original data in Section 7. In Section 8, we analyze the horizontal distribution of modal expansion coefficients. As a by-product of this analysis, we will map the coefficients onto a regular grid over the entire ocean resulting in a simple description of the hydrography of the whole North Atlantic Ocean. A summary of the findings from this chapter and a conclusion where the results are put into perspective are given in sections 8 and 9 respectively.

\section{Data Description}

The hydrographic data used in this study are from nine different experiments involving many investigators. The different data sets and cruises are listed in Table $2-1$. The spatial distribution of these stations were plotted in Figure 2-1. The data we use consist of CTD- $\mathrm{O}_{2}$ data (temperature, salinity, oxygen) and bottle nutrients (phosphate, silicate, nitrate) from all cruises except TOPOGULF. All data were interpolated to standard depths as was described in Section 3 of the previous chapter.

TOPOGULF is a Franco-German experiment initiated by researchers at IFREMER/Brest and IFM/Kiel (TOPOGULF group, 1986). Of this survey about the midlatitude Mid-Atlantic ridge, we only use the hydrographic data from the southern portion occupied by R.V. Le Suroit. Furthermore, only the data from the CTD-O 2 instrument is used because the available nutrient data was incomplete (missing depths and missing stations).

The TTO (Transient Tracers in the Ocean) program was an experiment to survey distributions of man-made tracers in the ocean, lead by a group of investigators (Brewer et al., 1985). The surveys were carried out from R.V. Knorr in two parts; North Atlantic Study (NAS), (PACODF, 1986a) and Tropical Atlantic Study (TAS) (PACODF, 1986b). A series of scientific papers resulting from this experiment are collected in Journal of Geophysical Research volume 90 numbers C4 and C5 (1985). 
Brief descriptions of the general hydrography of other sections are given below. (A description of Endeavor $129\left(64^{\circ} \mathrm{W}\right)$ was presented in Chapter 1.) The purpose here is to review the general hydrographic structures of the North Atlantic Ocean which will facilitate later analysis in this chapter by providing a background reference. The contour plots are interesting in their own right. All observations are modern; they are all occupied between 1981 to 1983 (except Endeavor 129 in 1985). Almost all hydrographic stations sample the whole water column from the surface to close to the sea floor and consist of a complete set of high quality observations of the major hydrographic variables; temperature, salinity, oxygen, phosphate, silicate, and nitrate. The combined data set thus provides a unique basis for analysis that is modern, of high quality, and of extensive spatial coverage. Presenting the data simultaneously is enlightening and helpful for future analysis.

\section{A. Oceanus $139 \operatorname{leg} 7-59^{\circ} \mathrm{W}$}

The observations were carried out by a group from the Woods Hole Oceanographic Institution (WHOI). This section (M.McCartney, Chief Scientist) extends from the coast of New Foundland to Brazil. A cruise report is given by Knapp and Stommel (1985). Contour plots of the six hydrographic variables are shown in Figure 2-2. This section traverses north-south close to the center of the subtropical gyre. We can identify several characteristics in these contour plots similar to features in the section along $64^{\circ} \mathrm{W}$ (Endeavor 129, Figure 1-2). The Gulf Stream is seen as the deepening of the isolines southward in the upper water column $(<1000 \mathrm{dbar})$ in all properties at about $38^{\circ} \mathrm{N}$. The thermocline and halocline rise gradually to the south which reflects the westward return flow of the wind driven anticyclonic circulation. Antarctic Intermediate Water is seen as the low salinity, low oxygen, high nutrient tongue centered about 700 dbar extending from the southern end to about $25^{\circ} \mathrm{N}$. The extent and relative contrast of the Antarctic Intermediate Water tongue with its ambient water is stronger in this section 
(a)
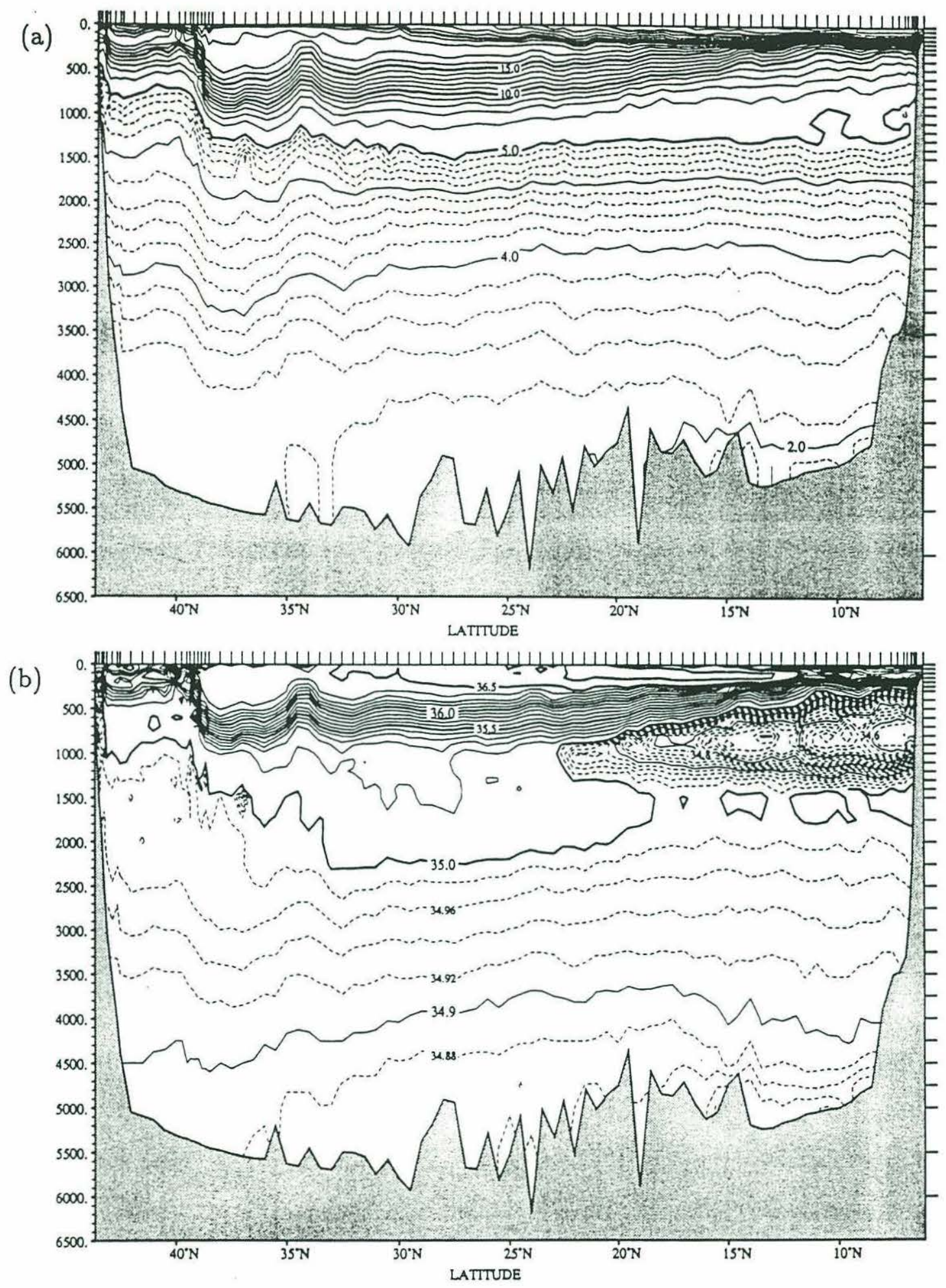

Figure 2-2. Oceanus 133 leg $7-53^{\circ} \mathrm{W}$

(a) Temperature $\left({ }^{\circ} \mathrm{C}\right)$ (b) Salinity (PSU) 

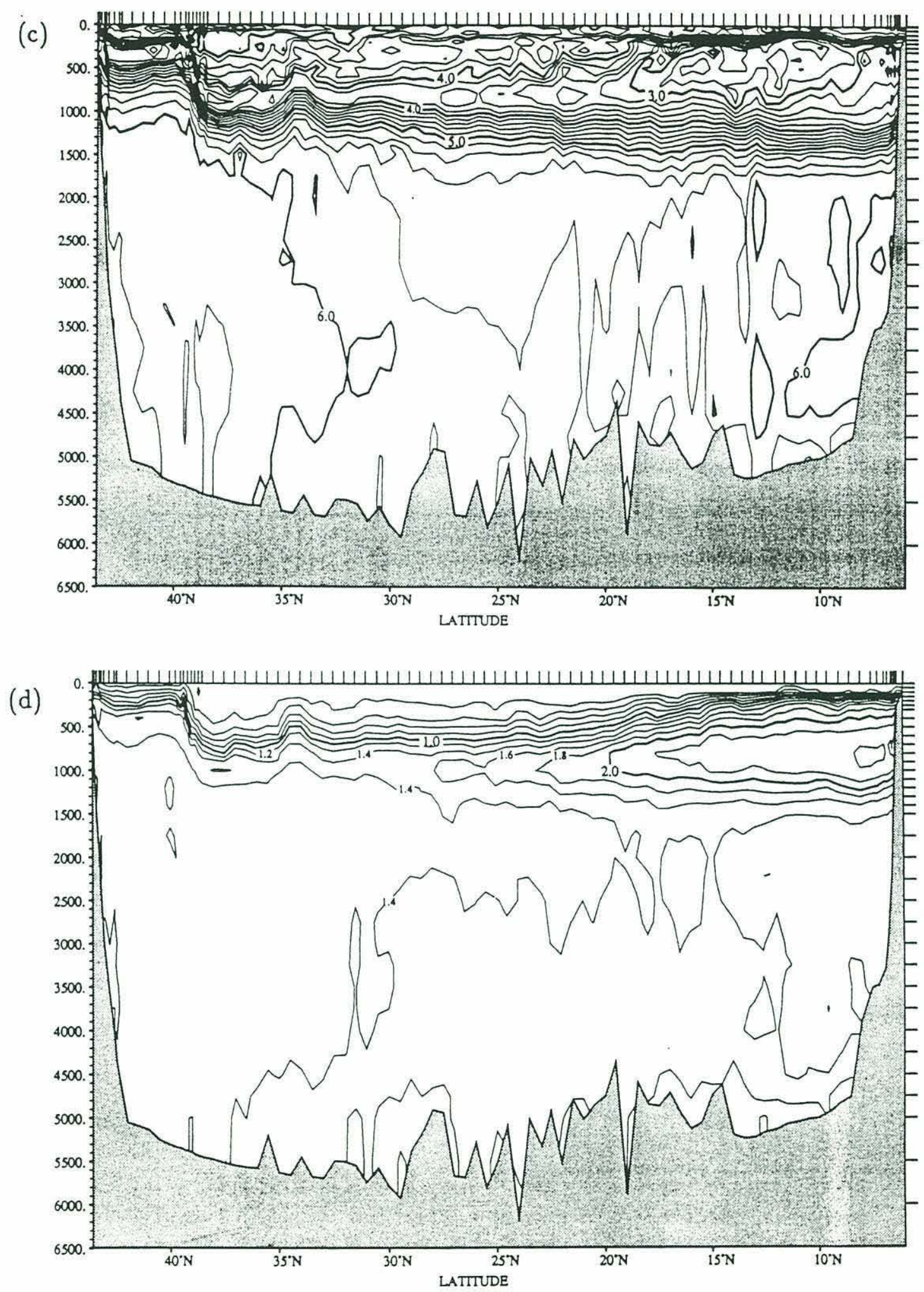

Figure 2-2. (continued) Oceanus 133 leg $7-53^{\circ} \mathrm{W}$

(c) Oxygen $(\mathrm{ml} / \mathrm{l})(\mathrm{d})$ Phosphate $(\mu \mathrm{mol} / \mathrm{kg})$ 

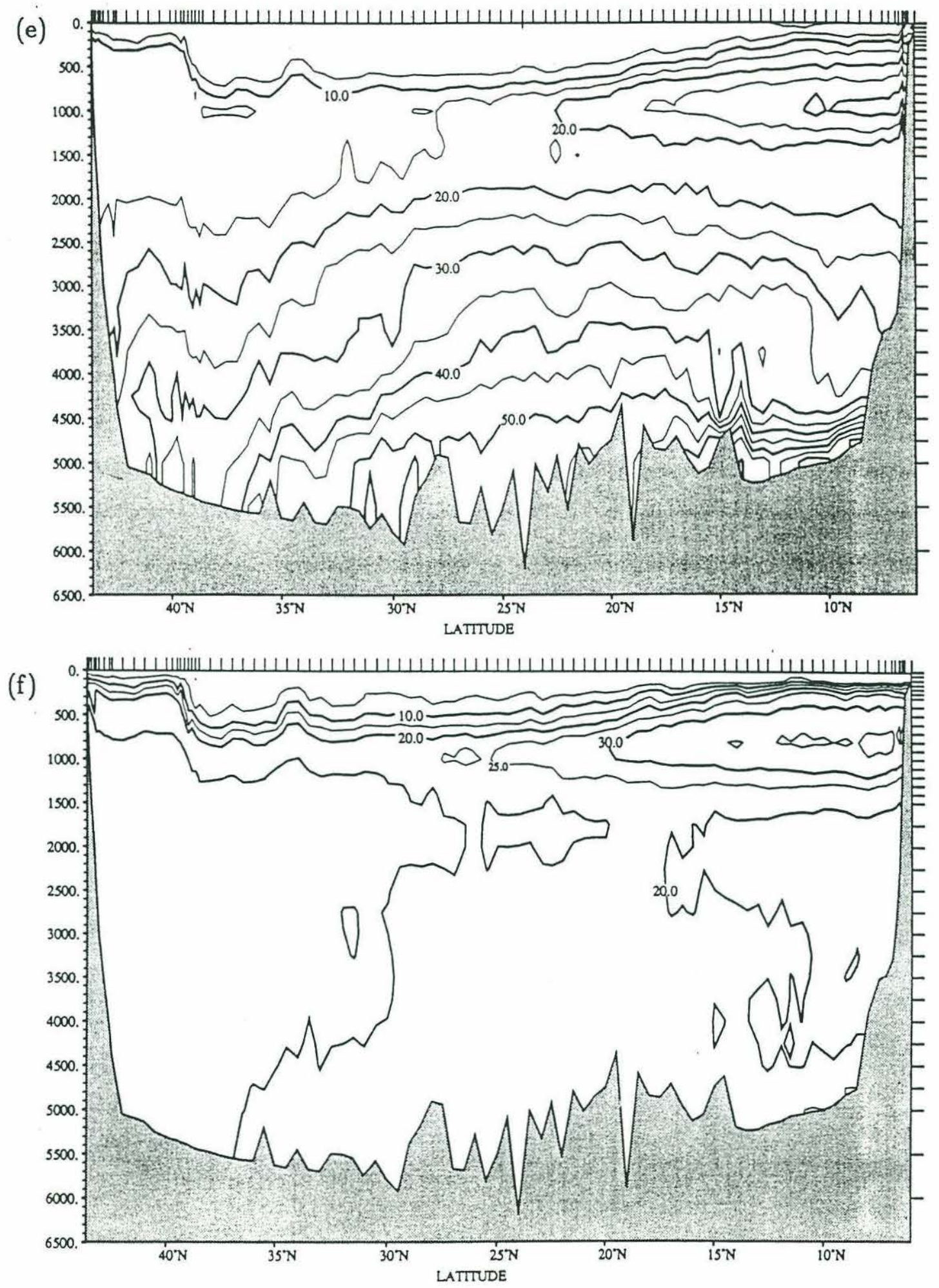

Figure 2-2. (continued) Oceanus 133 leg $7-53^{\circ} \mathrm{W}$

(e) Silicate $(\mu \mathrm{mol} / \mathrm{kg})$ (f) Nitrate $(\mu \mathrm{mol} / \mathrm{kg})$ 
than was seen in Endeavor 129. The effect of the high salinity Mediterranean Water is seen beneath the halocline at mid latitude $\left(\sim 25^{\circ} \mathrm{N}\right)$, as the downward bulge of the 35.1 PSU contour.

\section{B. Knorr $104-35^{\circ} \mathrm{W}$}

This cruise was also carried out by a group from WHOI. The section (M.McCartney, Chief Scientist) extends from the southern end of Greenland to Recife, Brazil. This hydrographic section is the longest of the three north-south sections used in this study, and contains the largest variety of different hydrographic regions in one section. Contour plots of properties are given in Figure 2-3. The northern part of this section lies in the western half of the subpolar gyre. The section crosses the Mid Atlantic Ridge into the eastern basin at midlatitudes $\left(\sim 35^{\circ} \mathrm{N}\right)$, and crosses the ridge back into the western basin in the tropics $\left(\sim 8^{\circ} \mathrm{N}\right)$.

In the subpolar region, the thermocline and halocline are absent and vertical gradient of the properties are much weaker than at lower latitudes. South of $50^{\circ} \mathrm{N}$, the isolines have a series of sharp undulations which are associated with the North Atlantic Current and its eddies and meanders. At mid latitudes, the thermocline forms a "bowl" shape of the subtropical gyre, beneath which there is the bulge of the high salinity Mediterranean outflow. Low salinity high nutrient Antarctic Intermediate Water is again evident at about 800 dbar towards the southern end of the section.

\section{Hudson $82-48^{\circ} \mathrm{N}$}

This is an east-west section extending from the Grand Banks to just south of Ireland. This section was carried out by a group from the Bedford Institute of Oceanography (BIO), lead by R.Hendry. The property contours, shown in Figure 2-4, display a contrast in the general hydrographic structure between the east and west of the Mid Atlantic Ridge $\left(\sim 30^{\circ} \mathrm{W}\right)$. At depth (below 2500 dbar) the eastern basin has higher nutrients, lower oxygen, and is warmer and more saline than the waters in the western 

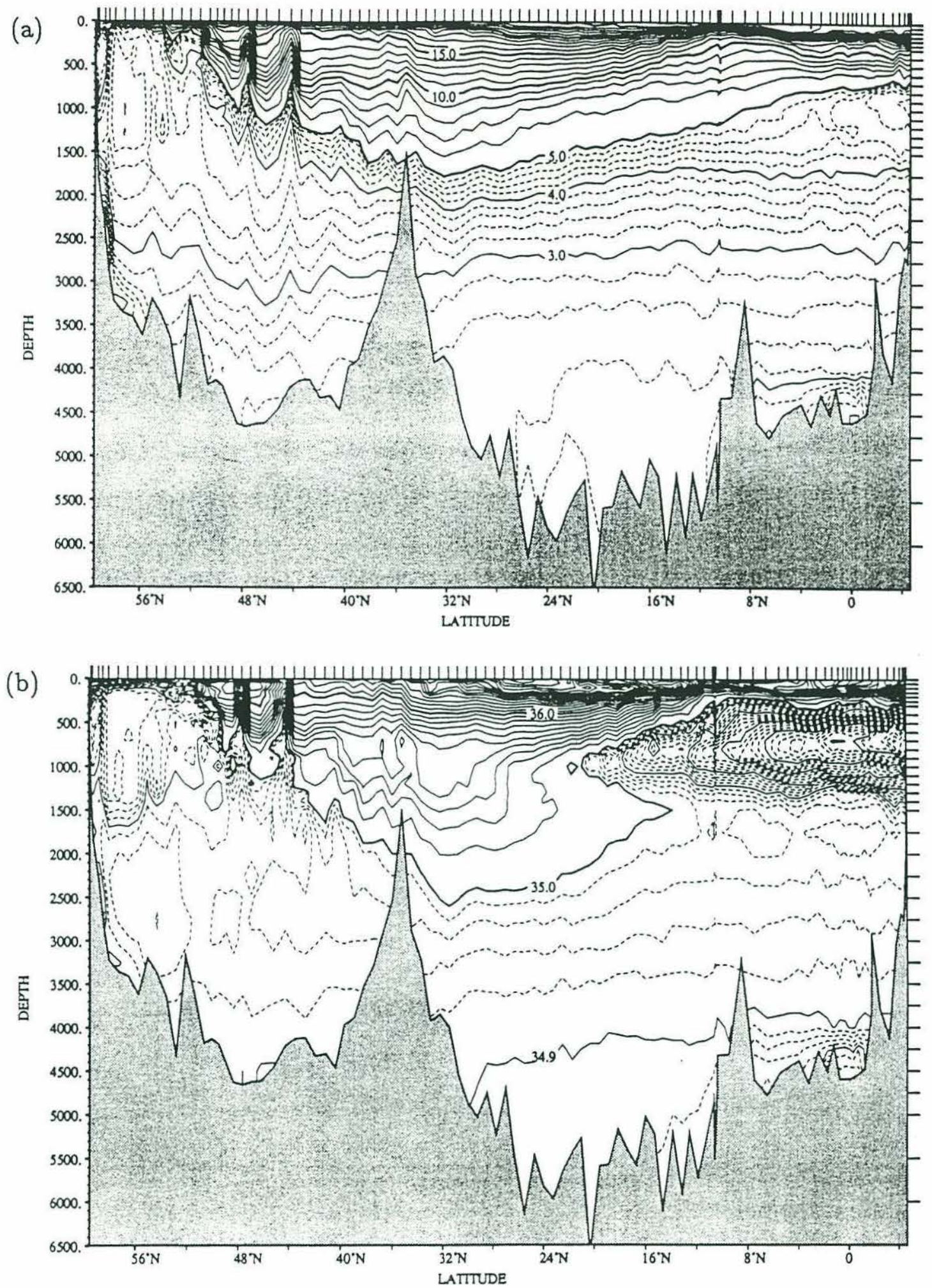

Figure 2-3. Contoured Data of Knorr $104-35^{\circ} \mathrm{W}$

(a) Temperature $\left({ }^{\circ} \mathrm{C}\right)$ (b) Salinity (PSU) 

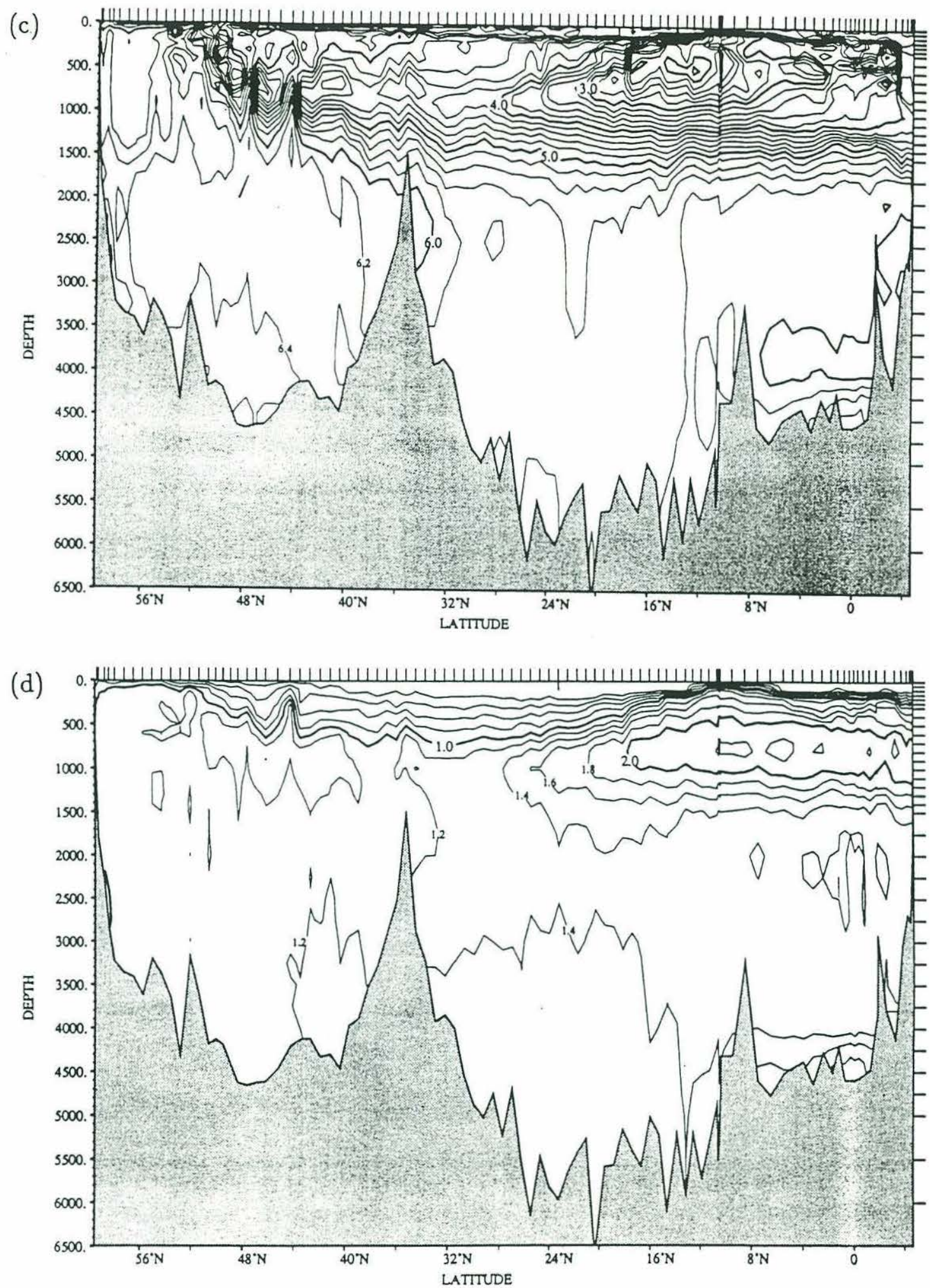

Figure 2-3. (continued) Knorr $104-35^{\circ} \mathrm{W}$

(c) Oxygen $(\mathrm{ml} / \mathrm{l})(\mathrm{d})$ Phosphate $(\mu \mathrm{mol} / \mathrm{kg})$ 

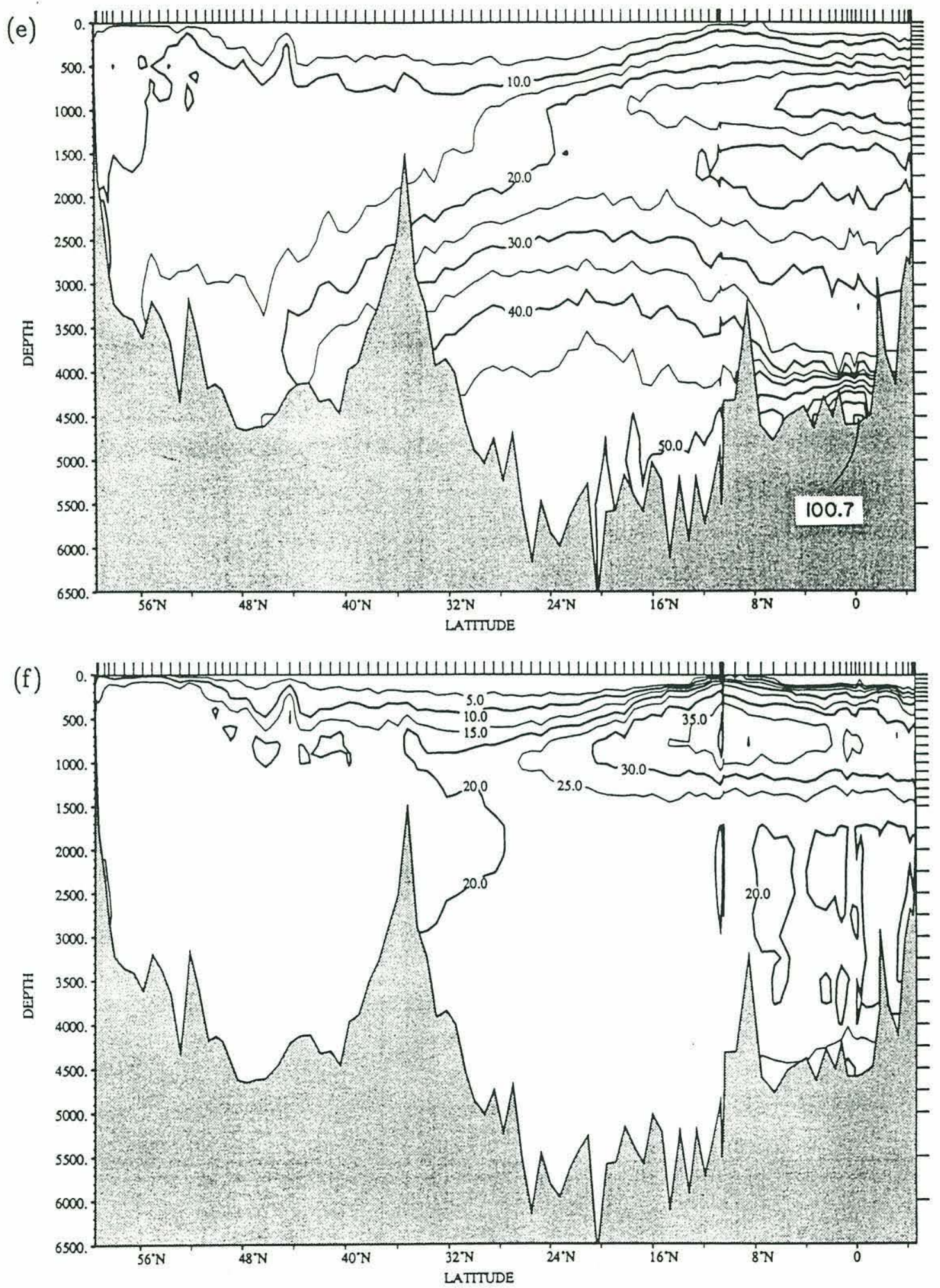

Figure 2-3. (continued) Knorr $104-35^{\circ} \mathrm{W}$

(e) Silicate $(\mu \mathrm{mol} / \mathrm{kg})$ (f) Nitrate $(\mu \mathrm{mol} / \mathrm{kg})$ 

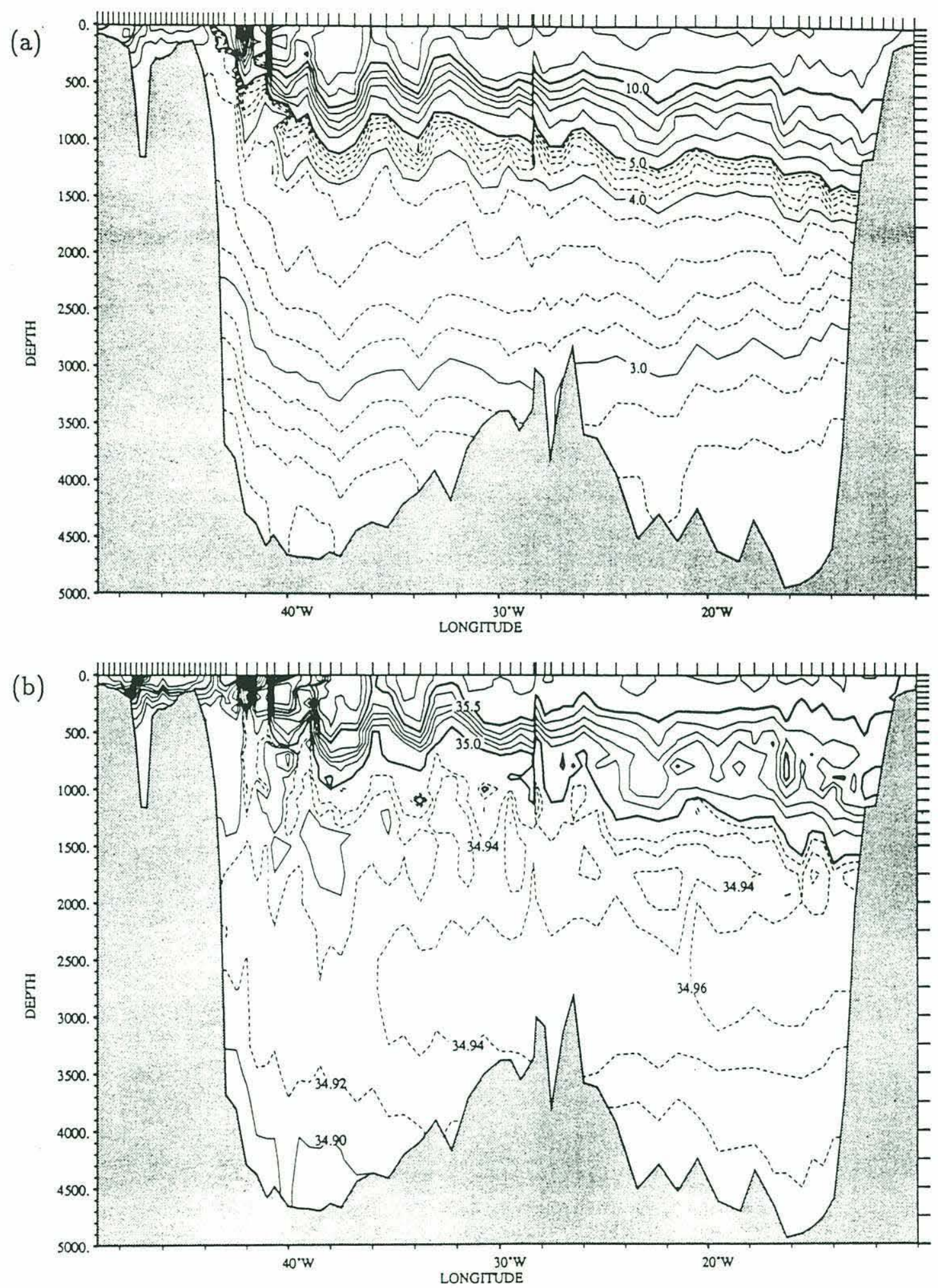

Figure 2-4. Contoured Data of Hudson $82-48^{\circ} \mathrm{N}$

(a) Temperature $\left({ }^{\circ} \mathrm{C}\right)(\mathrm{b})$ Salinity (PSU) 

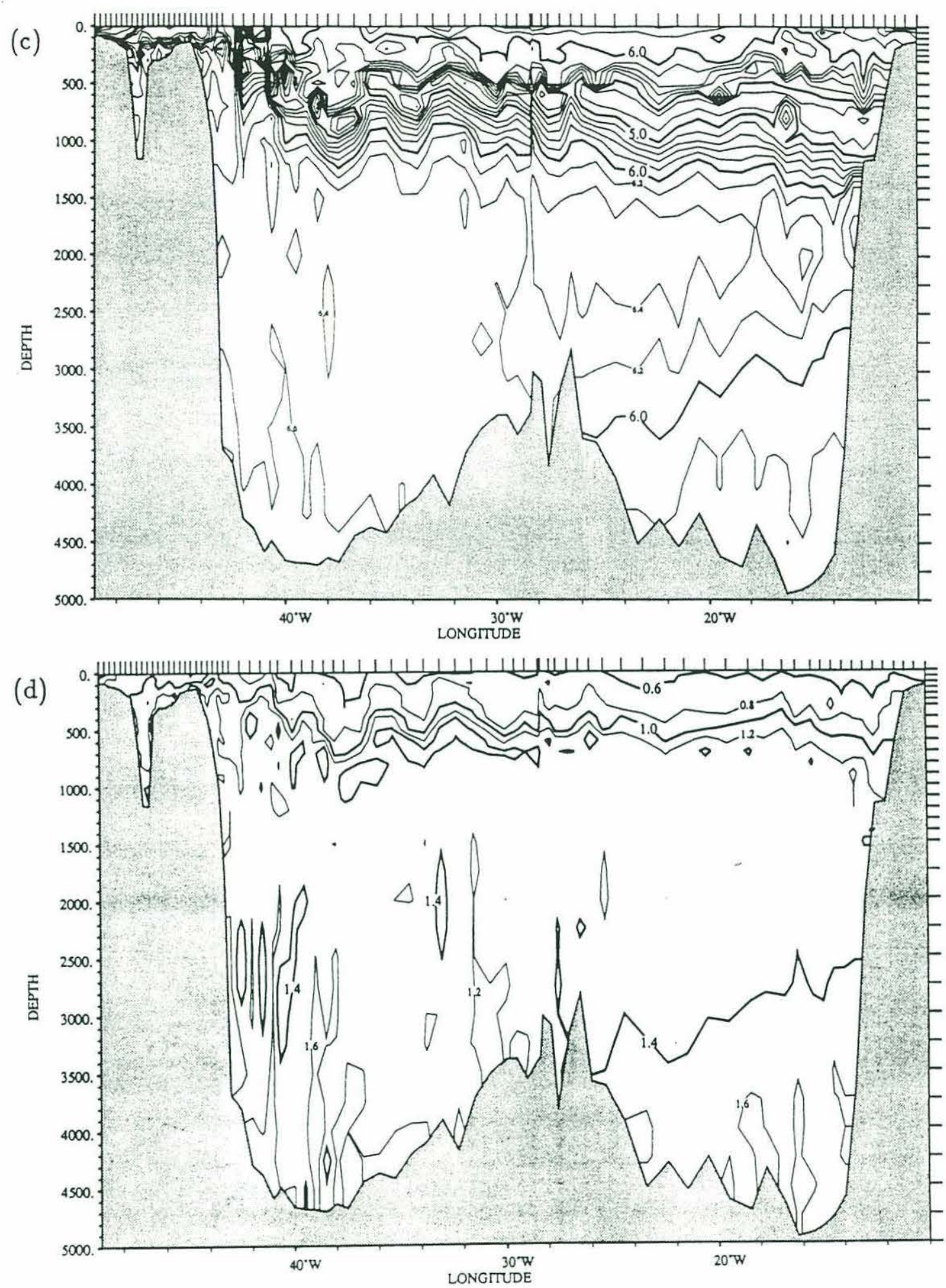

Figure 2-4. (continued) Hudson $82-48^{\circ} \mathrm{N}$ (c) Oxygen $(\mathrm{ml} / \mathrm{l})(\mathrm{d})$ Phosphate $(\mu \mathrm{mol} / \mathrm{kg})$ 

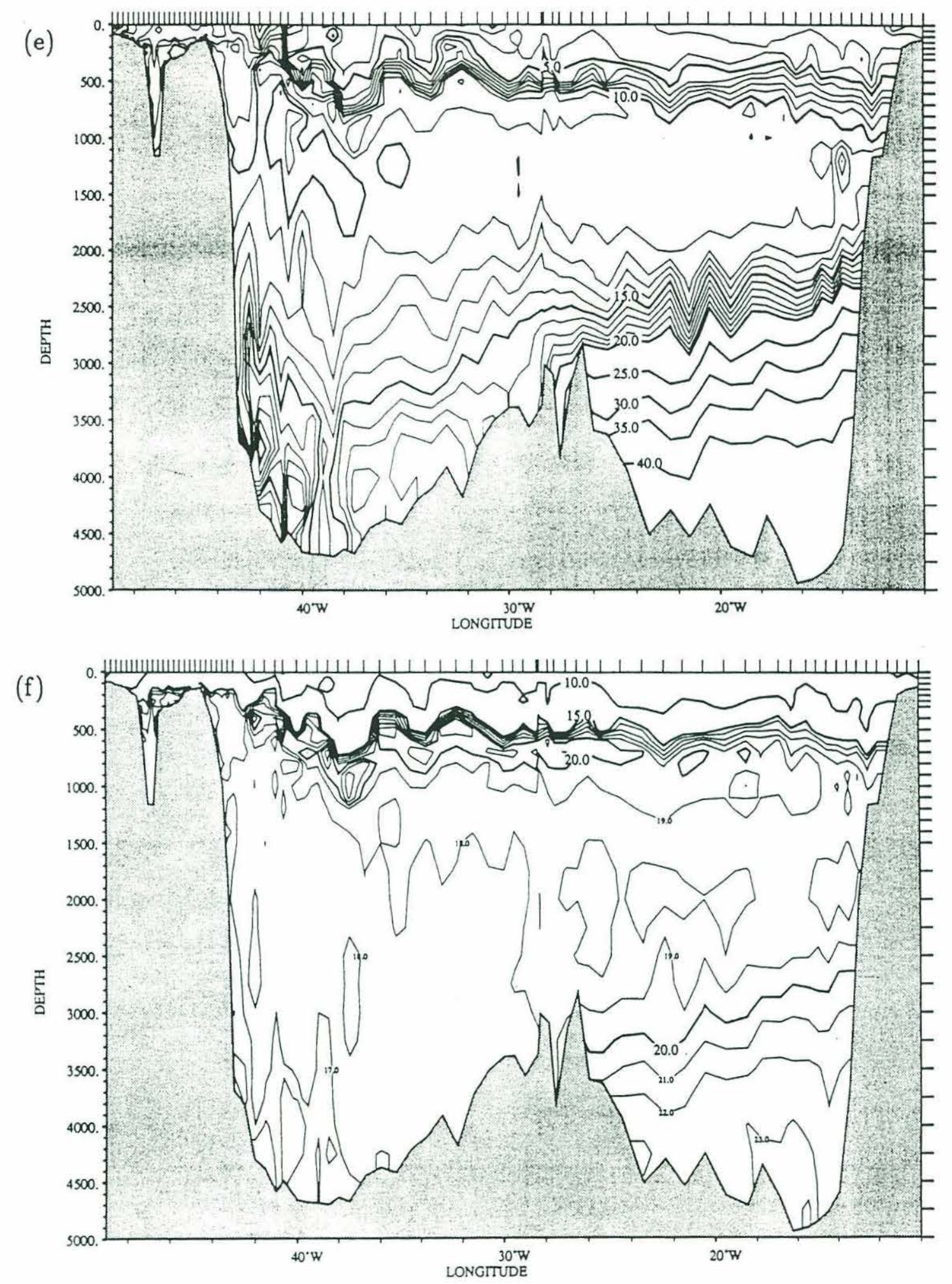

Figure 2-4. (continued) Hudson $82-48^{\circ} \mathrm{N}$ (e) Silicate $(\mu \mathrm{mol} / \mathrm{kg})$ (f) Nitrate $(\mu \mathrm{mol} / \mathrm{kg})$ 
basin. This reflects the contrast between the higher concentration of North Atlantic Deep Water in the western basin to the higher concentration of Antarctic origin water in the eastern basin. At shallower depths between $500 \mathrm{dbar}$ and $1500 \mathrm{dbar}$, the eastern basin is more saline than the western basin which reflects the influence of Mediterranean outflow in the eastern half. The North Atlantic Current $\left(\sim 40^{\circ} \mathrm{W}\right)$ and its associated eddies $\left(40^{\circ} \mathrm{W} \sim 30^{\circ} \mathrm{W}\right)$ are seen as the short horizontal undulations of the thermocline in the western basin, whereas the eastern basin is relatively quiescent.

\section{Atlantis II $109 \operatorname{leg} 1-36^{\circ} \mathrm{N}$}

This section and leg 3 of the same cruise described below (E.) were described by Roemmich and Wunsch (1985). This east-west section at $36^{\circ} \mathrm{N}$ (C.Wunsch, Chief Scientist) extends from just north of Cape Hatteras to southeast of Cape St. Vincent, cutting across the subtropical gyre towards its northern end. Property contours are shown in Figure 2-5. The Gulf Stream is at $70^{\circ} \mathrm{W}$ as evidenced by the steeply sloping isotherms. The short horizontal scale upward bulge at $66^{\circ} \mathrm{W}$ is a cold-core ring. The upper thermocline and halocline shoal to the east reflecting the southward interior flow of the subtropical gyre. At the eastern end, we see the high salinity Mediterranean Water centered about $1200 \mathrm{dbar}$. The influence of these high salinity waters is seen to extend westward to at least $40^{\circ} \mathrm{W}$. The deep waters exhibit a east-west contrast about the Mid Atlantic Ridge, similar to the $48^{\circ} \mathrm{N}$ section.

\section{E. Atlantis II 109 leg $3-24^{\circ} \mathrm{N}$}

This east-west section, which runs from the Bahamas to the Canary Islands, is situated towards the southern end of the subtropical gyre. Property contour plots are shown in Figure 2-6. The hydrographic variation is much weaker than previous sections, but still shows a gradual rise of the thermocline to the east reflecting the subtropical circulation. The influence of the high salinity Mediterranean Water is still evident towards the eastern end of the section at about $1000 \mathrm{dbar}$. 

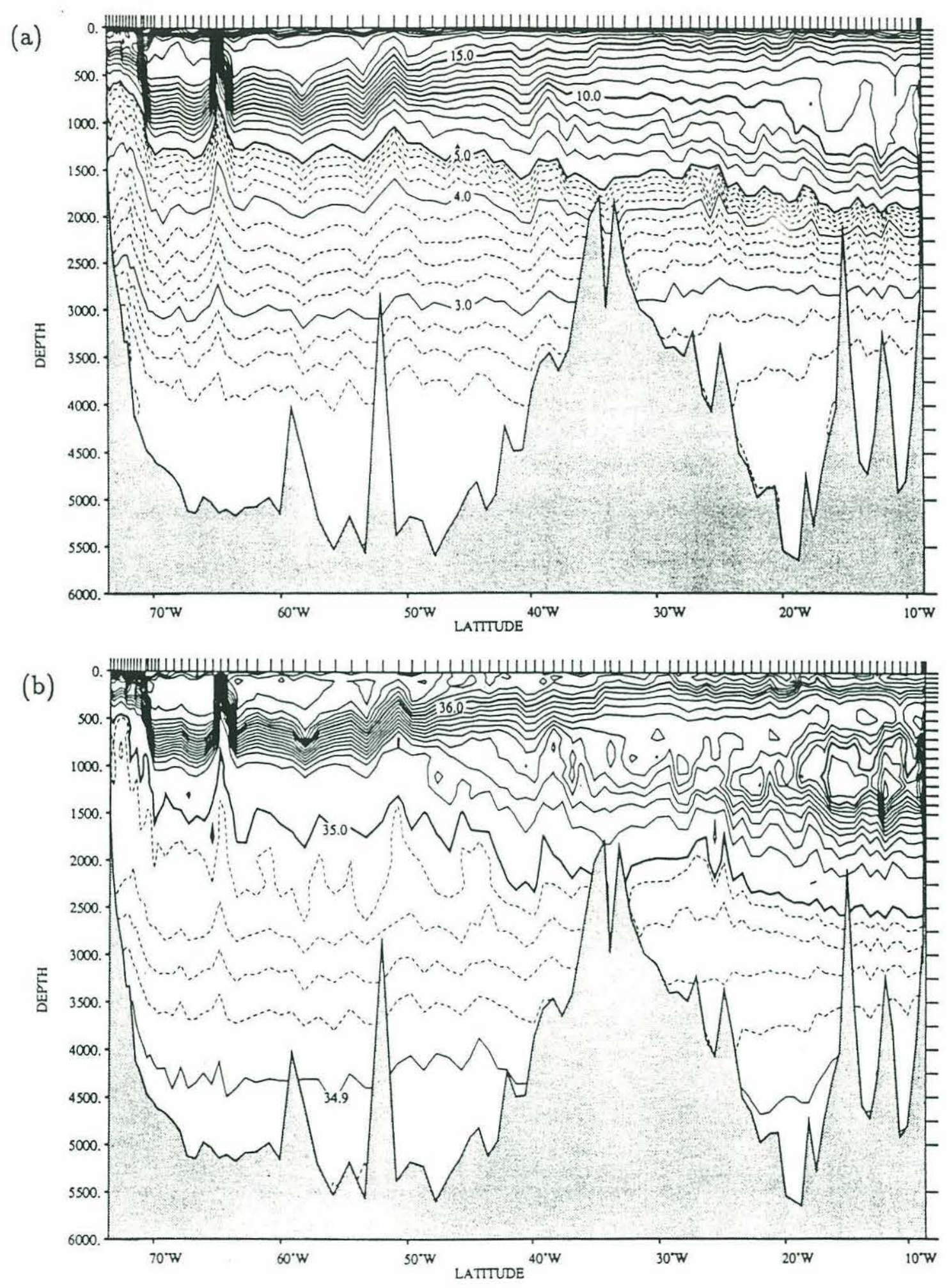

Figure 2-5. Contoured Data of Atlantis II $-36^{\circ} \mathrm{N}$

(a) Temperature $\left({ }^{\circ} \mathrm{C}\right)$ (b) Salinity (PSU) 

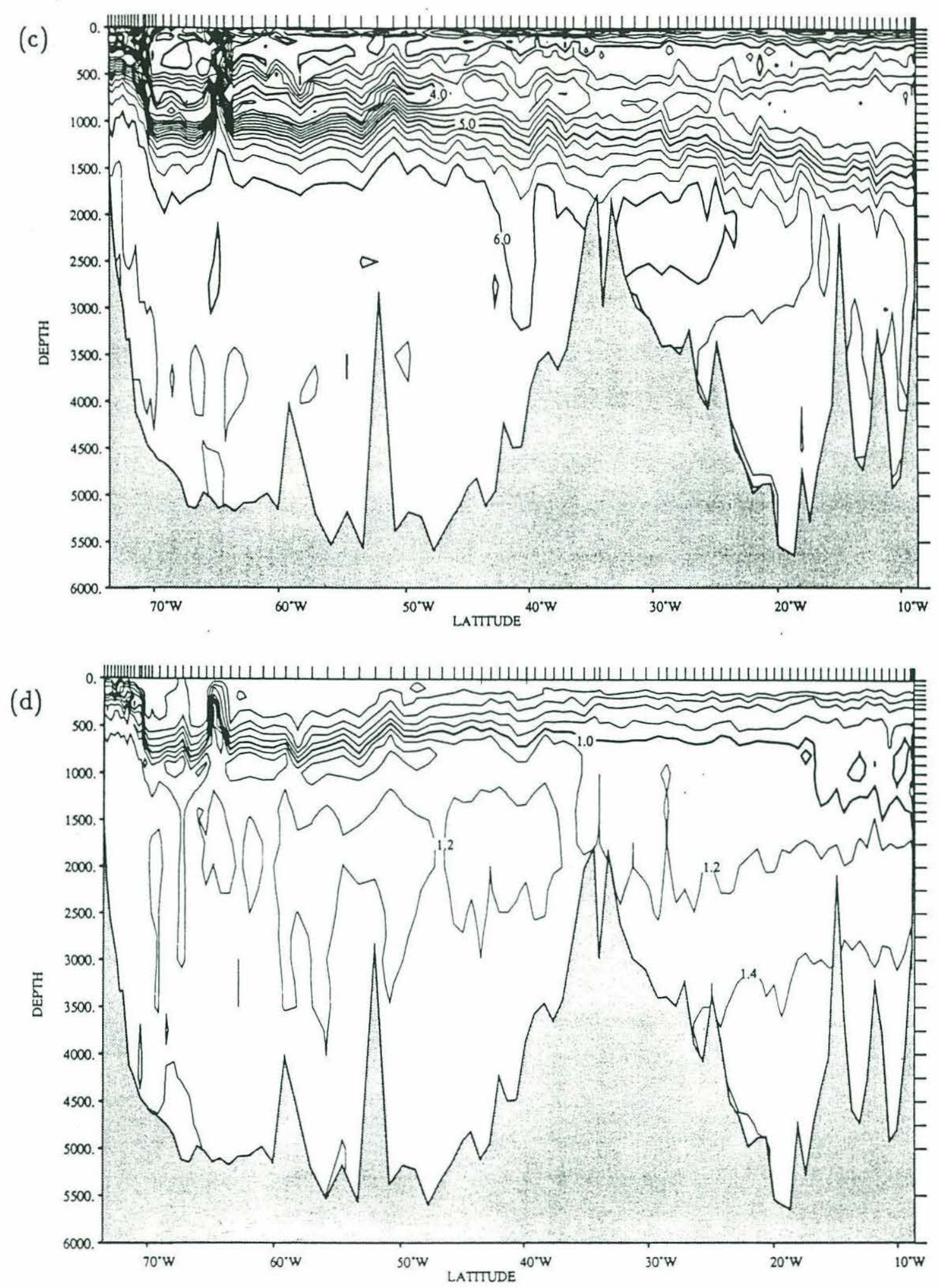

Figure 2-5. (continued) Atlantis II $-36^{\circ} \mathrm{N}$

(c) Oxygen $(\mathrm{ml} / \mathrm{l})(\mathrm{d})$ Phosphate $(\mu \mathrm{mol} / \mathrm{kg})$ 

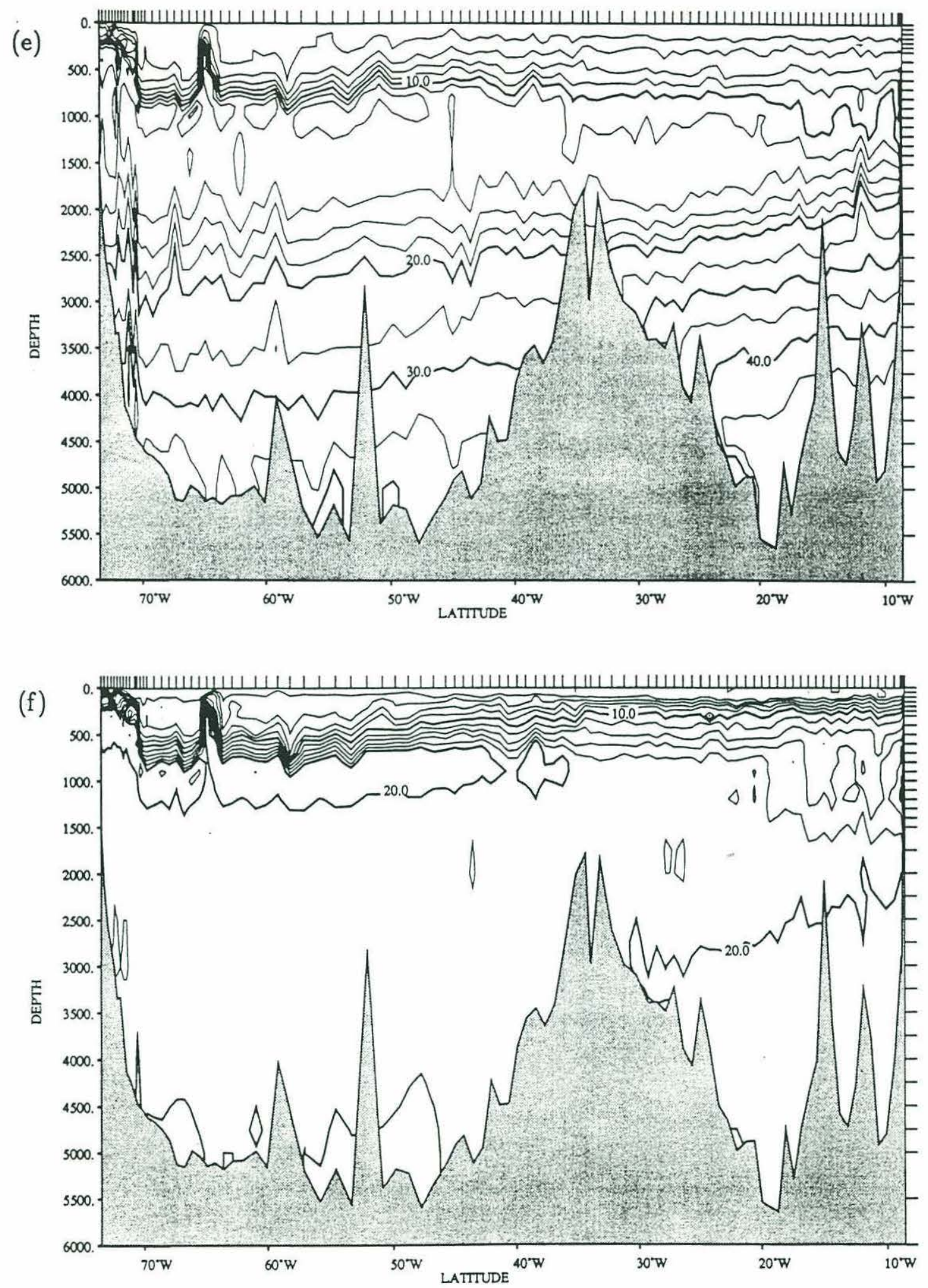

Figure 2-5. (continued) Atlantis II $-36^{\circ} \mathrm{N}$

(e) Silicate $(\mu \mathrm{mol} / \mathrm{kg})$ (f) Nitrate $(\mu \mathrm{mol} / \mathrm{kg})$ 

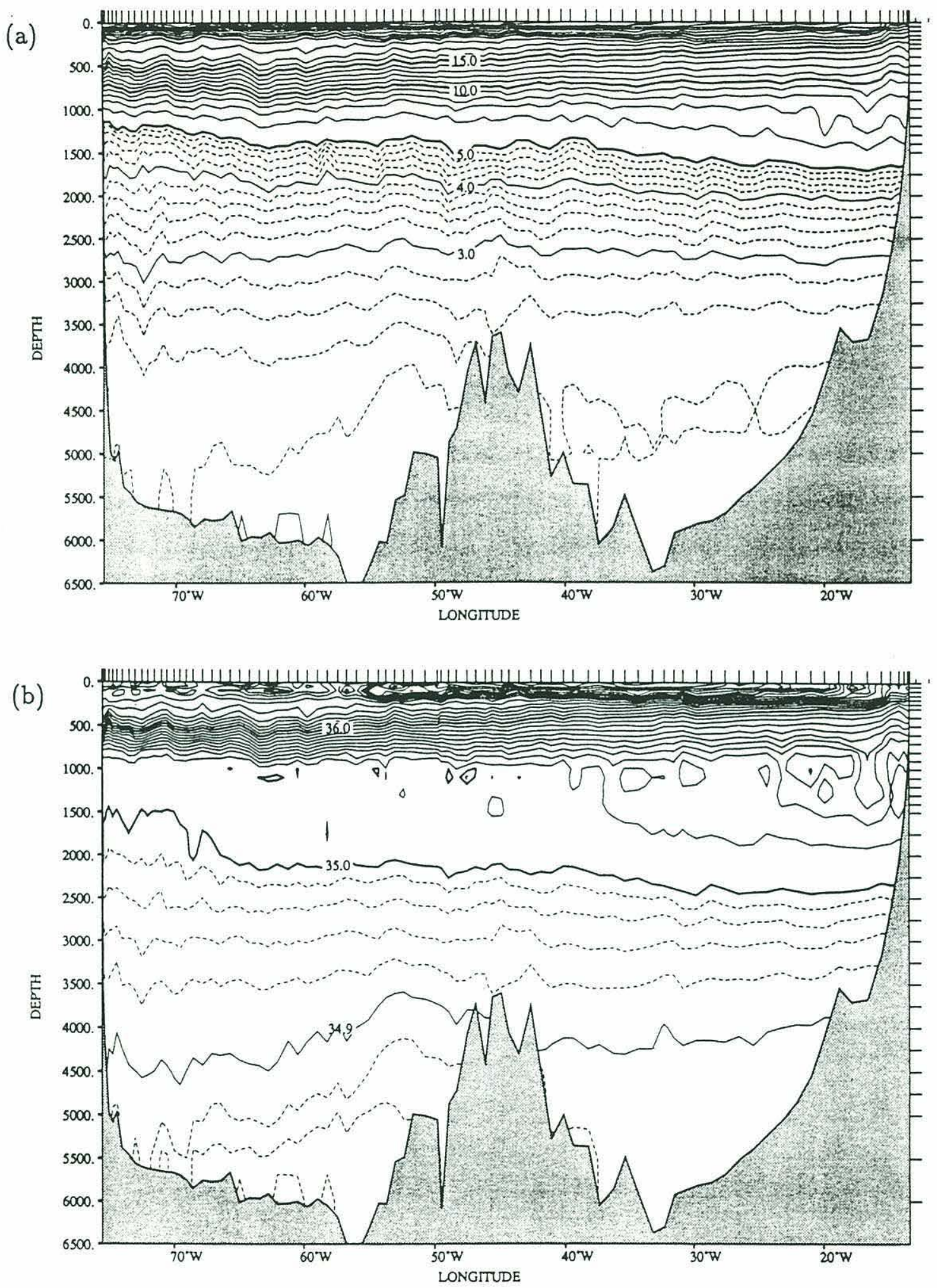

Figure 2-6. Contoured Data of Atlantis II $-24^{\circ} \mathrm{N}$

(a) Temperature $\left({ }^{\circ} \mathrm{C}\right)$ (b) Salinity (PSU) 

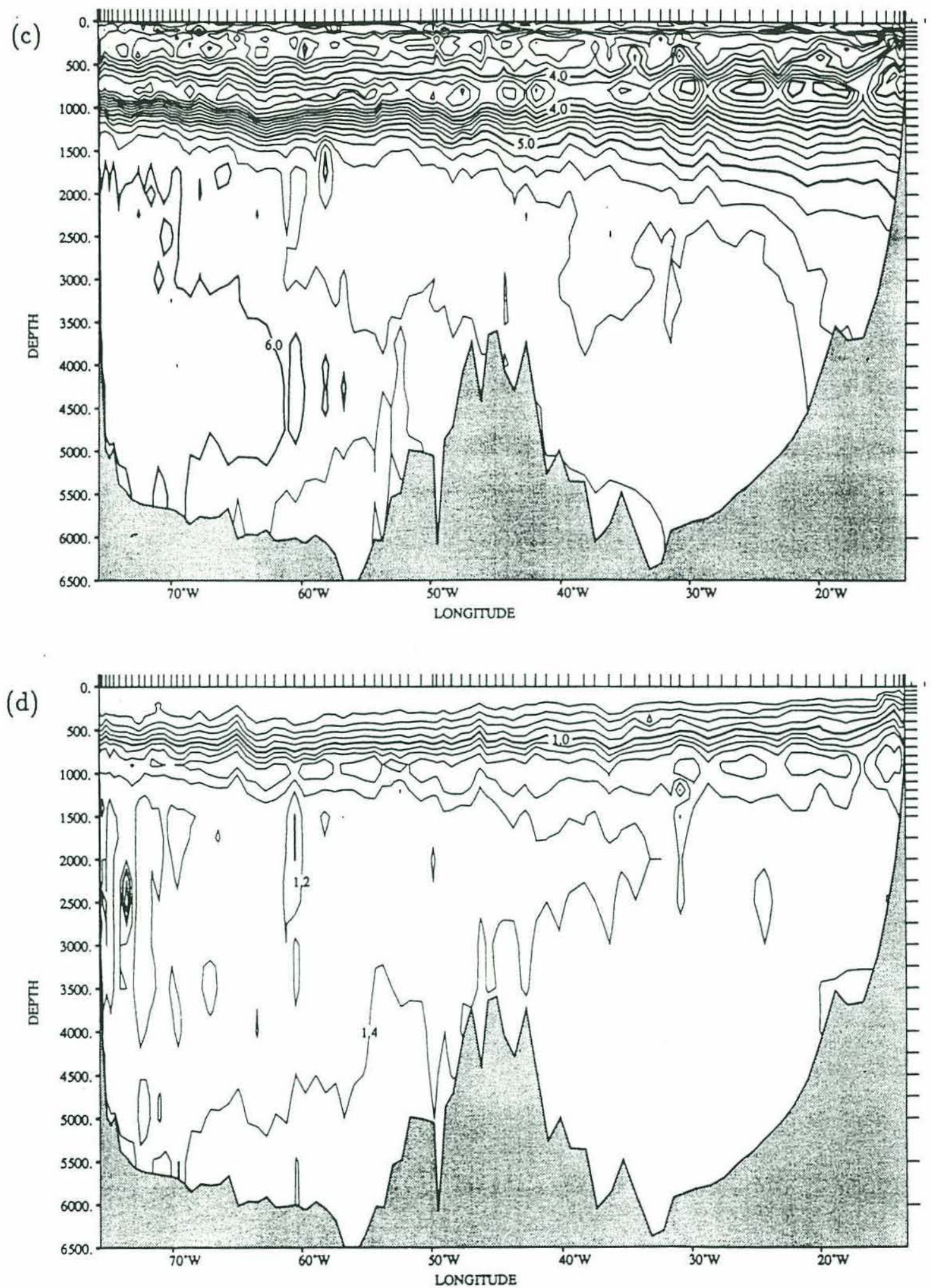

Figure 2-6. (continued) Atlantis II $-24^{\circ} \mathrm{N}$ (c) Oxygen (ml/l) (d) Phosphate $(\mu \mathrm{mol} / \mathrm{kg})$ 

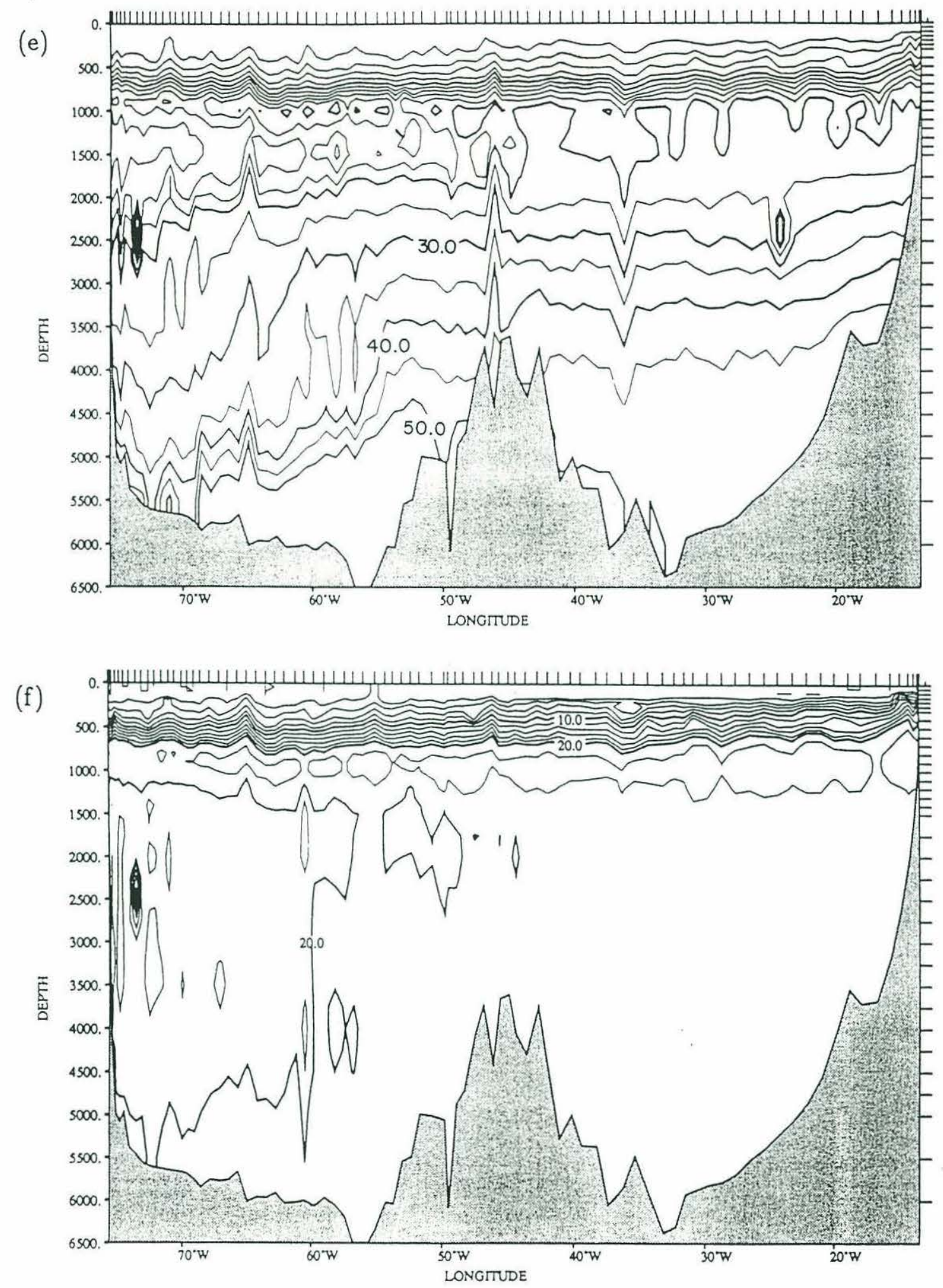

Figure 2-6. (continued) Atlantis II $-24^{\circ} \mathrm{N}$ (e) Silicate $(\mu \mathrm{mol} / \mathrm{kg})$ (f) Nitrate $(\mu \mathrm{mol} / \mathrm{kg})$ 


\section{Normalization}

\subsection{Range of Stations}

In examining the statistical characteristics of the entire North Atlantic Ocean, we must make sure that the stations are evenly distributed so that no one region is overrepresented in the statistics. For instance, if most of the stations were situated over a small region of the Mediterranean salt tongue, the modes will be tuned to the structure of this region since the structural variation of the tongue will be the dominant structural characteristic of the hydrography.

Wright and Worthington (1970) have divided the North Atlantic Ocean into six basins, based on deep water characteristics (Figure 2-7). They designated two northern basins (Labrador, European), two subtropical basins (North American, North African), and two tropical basins (Guiana, Guinea). We will use these basins as a reference for regional uniformity. To conform to the extent of the hydrographic section, we slightly modify the southern and northern basin boundaries as shown in Figure 2-7.

Among the 1085 stations, we exclude stations in the Norwegian Sea and Caribbean Sea from the calculation for the modes, since deep water characteristics at these stations are quite different from the typical North Atlantic and because there is no direct passage of these deep waters with the rest of the North Atlantic Ocean. We also exclude stations shallower than 1100 dbar (standard depth 15) such as stations in the Florida Straits and over Grand Banks. (Too shallow a station will put spurious structure below the maximum depth due to the normalization we perform; namely, the forcing of values below the maximum depth to the mean profile.) Deep water stations where there are less than 15 standard depths of data, because of missing bottle or because of a shallow cast, were also excluded from the analysis. Furthermore, some duplicate stations (like the ones near $32^{\circ} \mathrm{N} 26^{\circ} \mathrm{W}$ of TTONAS) were eliminated and some anomalous stations (for example where surface salinity is as low as $31.9 \mathrm{PSU}$ in surrounding waters of 34.5 PSU, possibly due to rain) were also excluded. This leaves a total of 895 stations to 


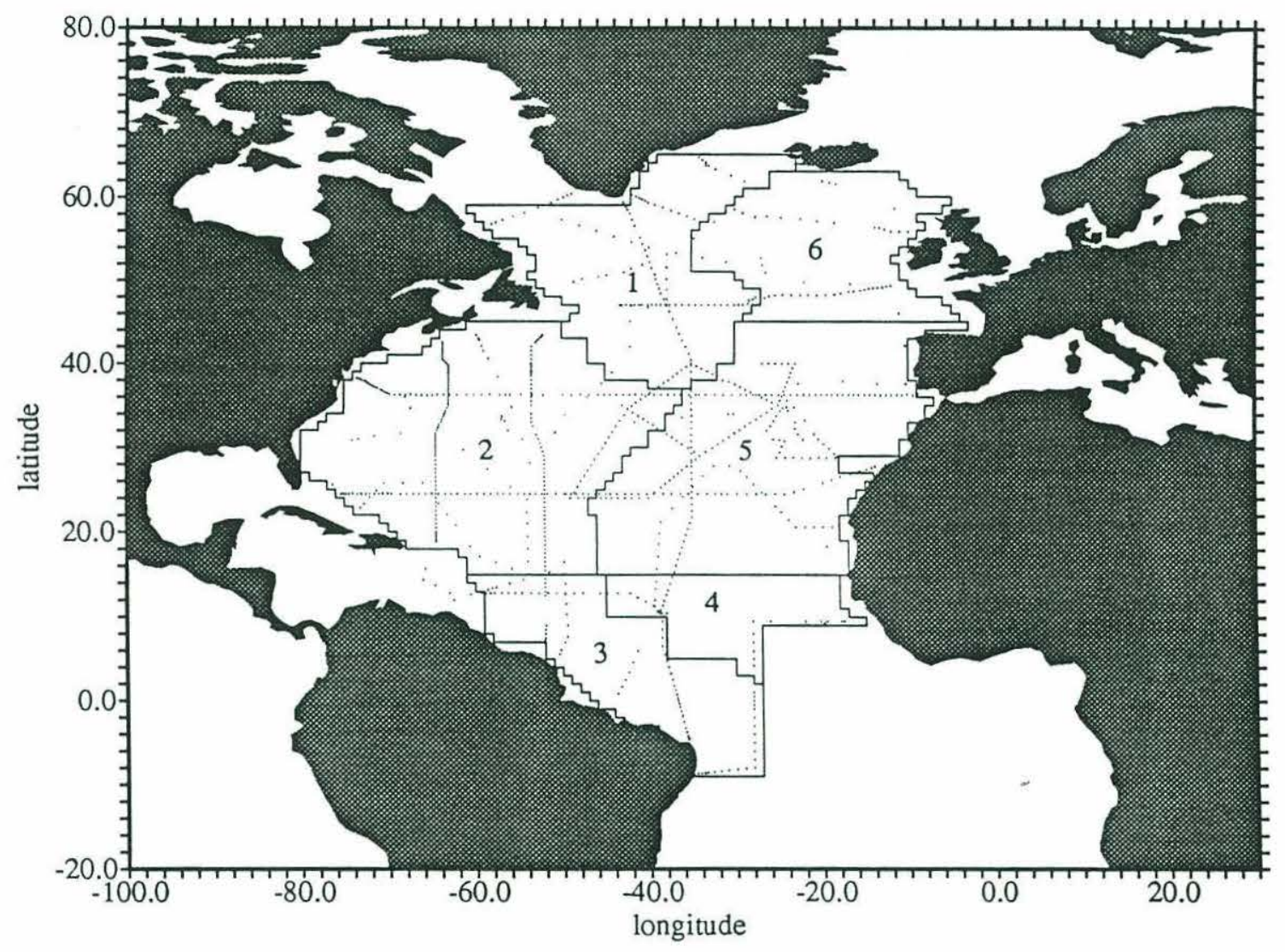

Figure 2-7. Basin Boundaries and Stations Used The basins are, from the one south of Greenland rotating counterclockwise, Labrador (basin 1), North American (2), Guiana (3), Guinea (4), North African (5), and European (6). 
analyze. These stations are plotted in Figure 2-7. The exact number of stations differ from property to property as some stations lack part of the data.

\subsection{Mean}

The data are normalized by their mean and significance estimate as was done in Chapter 1. The mean profile for the North Atlantic study was calculated by weighting each basin's mean profile with the area of the basin. For example,

$$
\bar{T}_{j}=\frac{\sum_{i} T_{j i} A_{i}}{\sum_{i} A_{i}},
$$

where $\bar{T}_{j}$ is the overall mean temperature at standard depth $j, T_{j i}$ is mean temperature at depth $j$ in basin $i$, and $A_{i}$ is the area of basin $i$. The basin mean profiles are simple averages within each basin. The maximum depth that we analyze is once again standard depth 35. The mean profiles of the six variables are shown in Figure 2-8.

\subsection{Normalization factor}

The weighting factor in equation (1-6) was calculated as in Chapter 1, but by averaging several sections: $64^{\circ} \mathrm{W}$ (Endeavor 129), $53^{\circ} \mathrm{W}$ (Oceanus $133 \mathrm{leg} 7$ ), $35^{\circ} \mathrm{W}$ (Knorr 104), $48^{\circ} \mathrm{N}$ (Hudson 82), $36^{\circ} \mathrm{N}$ (Atlantis II $109 \mathrm{leg}$ ), and $24^{\circ} \mathrm{N}$ (Atlantis II 109 leg 2). The values are calculated as a function of station separations along these sections. These measures of significance are also shown in Figure 2-8.

The mean station separation differs from section to section. However, we must emphasize once more that since we only need a relative measure of noise among different properties and different depths, such a simple evaluation of relative noise will suffice.

The overall root mean square difference is close to variations over five station separations in any of the hydrographic sections. The average distance of five station separations using the 6 trans-ocean sections is about $290 \mathrm{~km}$. So the measure shown in Figure 2-8 is likely an over estimate for mesoscale variability. A closer examination 
A. temperature $(\operatorname{deg} C)$

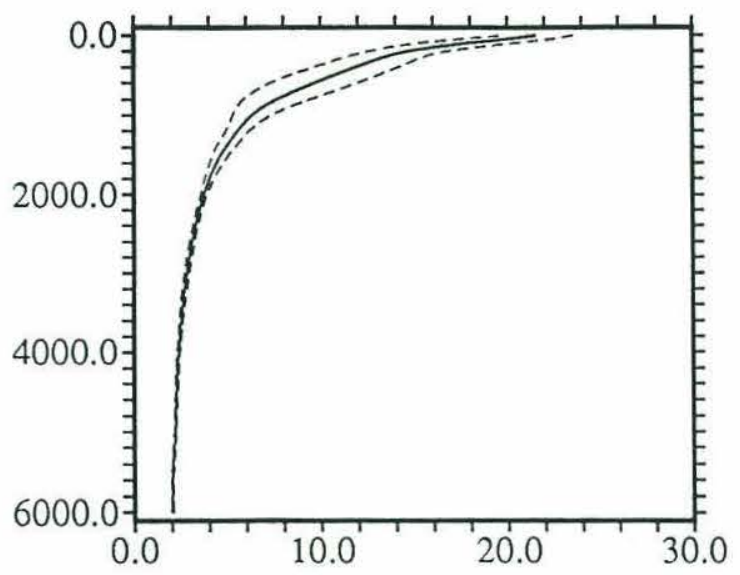

C. oxygen $(\mathrm{ml} / \mathrm{l})$

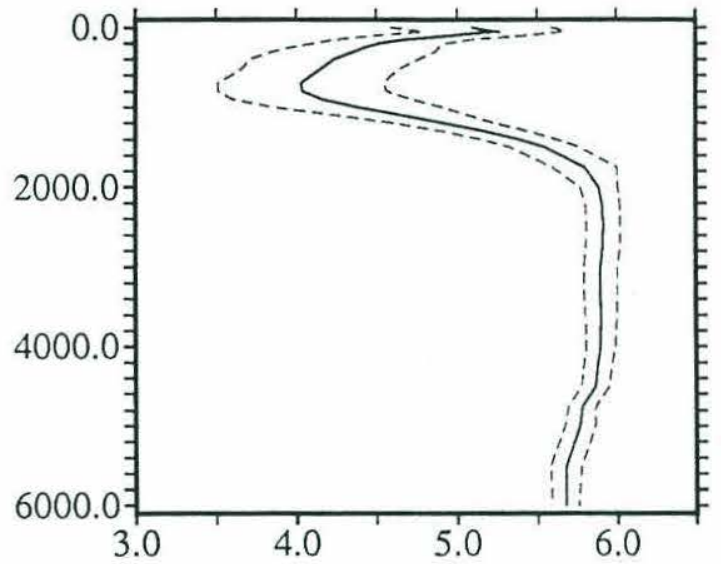

E. silicate (micro $\mathrm{mol} / \mathrm{kg}$ )

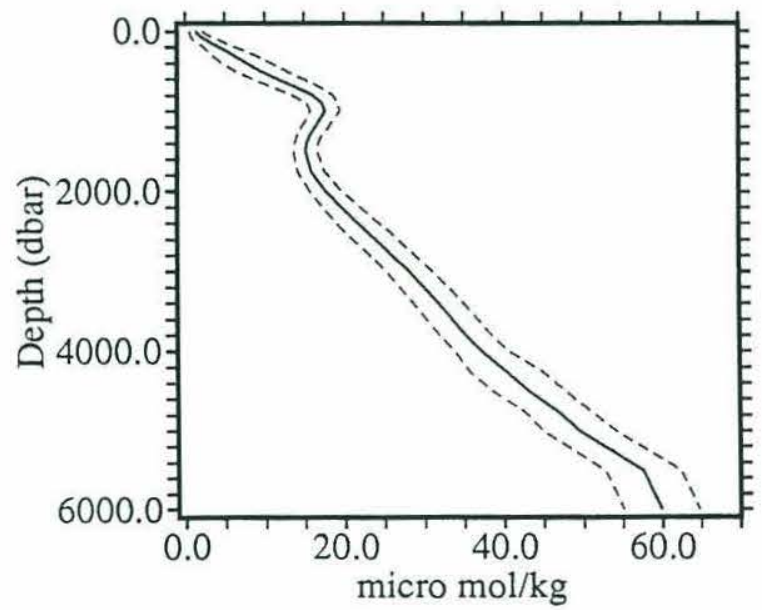

B. salinity (PSU)
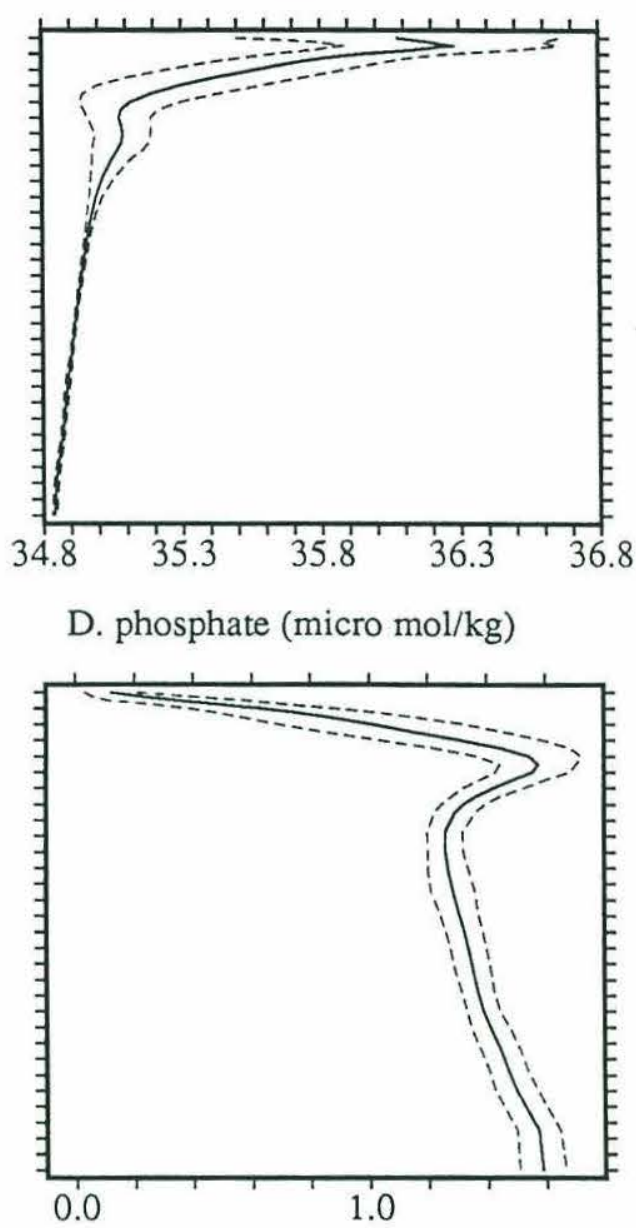

F. nitrate (micro $\mathrm{mol} / \mathrm{kg}$ )

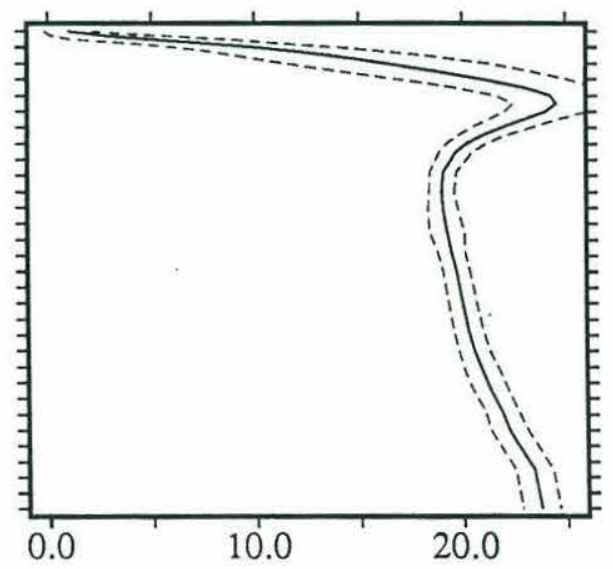

Figure 2-8. Normalization

The figures show mean (solid) plus/minus the normalization factor (dashed). 
of the rms difference shows that a variation over $100 \mathrm{~km}$ would halve the magnitude of these normalization factors used in this analysis.

\subsection{Areal Weighting}

Column weighting of the matrix $A$ (equation 1-10) is performed, so that the contribution from each basin to the covariance matrix, $A A^{T}$, is proportional to the area of that basin. Define a weighting factor $a_{i k}$, such that,

$$
\frac{\text { Area of Basin } i}{\text { \# of stations in basin for scalar } k \times a_{i k}} \text {, }
$$

is uniform among different basin i's and different properties $k$ 's. We then multiply each profile by $\sqrt{a}_{i k}$. The square root occurs because the covariance matrix is a quadratic sum of each profile. In the calculation, we chose to use a normalization such that equation (2-2) is equal to 1 for temperature in basin 2 . Note that, because of this column weighting to $A$, the expansion coefficients for the stations calculated as,

$$
V=\left(\Lambda^{-1} U^{T} A\right)^{T},
$$

will not be a set of orthonormal vectors. ( $U$ is the singular vector of the weighted matrix, $\left.A^{\prime}=A \cdot\left(\sqrt{a}_{i k}\right).\right)$

\section{Result of Modal Decomposition}

\subsection{Vertical Modes}

The first eight vertical modes are shown in Figure 2-9. The higher modes have increasingly more zero crossings, which was also the case in Chapter 1 . However, the structure of each mode is different from those in Chapter 1. Apparently, the modal structures depend upon the region of analysis. On the other hand, a visual inspection of the structure suggests that adding and subtracting the first 2 modes in Figure 2-9 gives a similar profile of the first two modes in the previous chapter (Figure 1-5, Chapter 


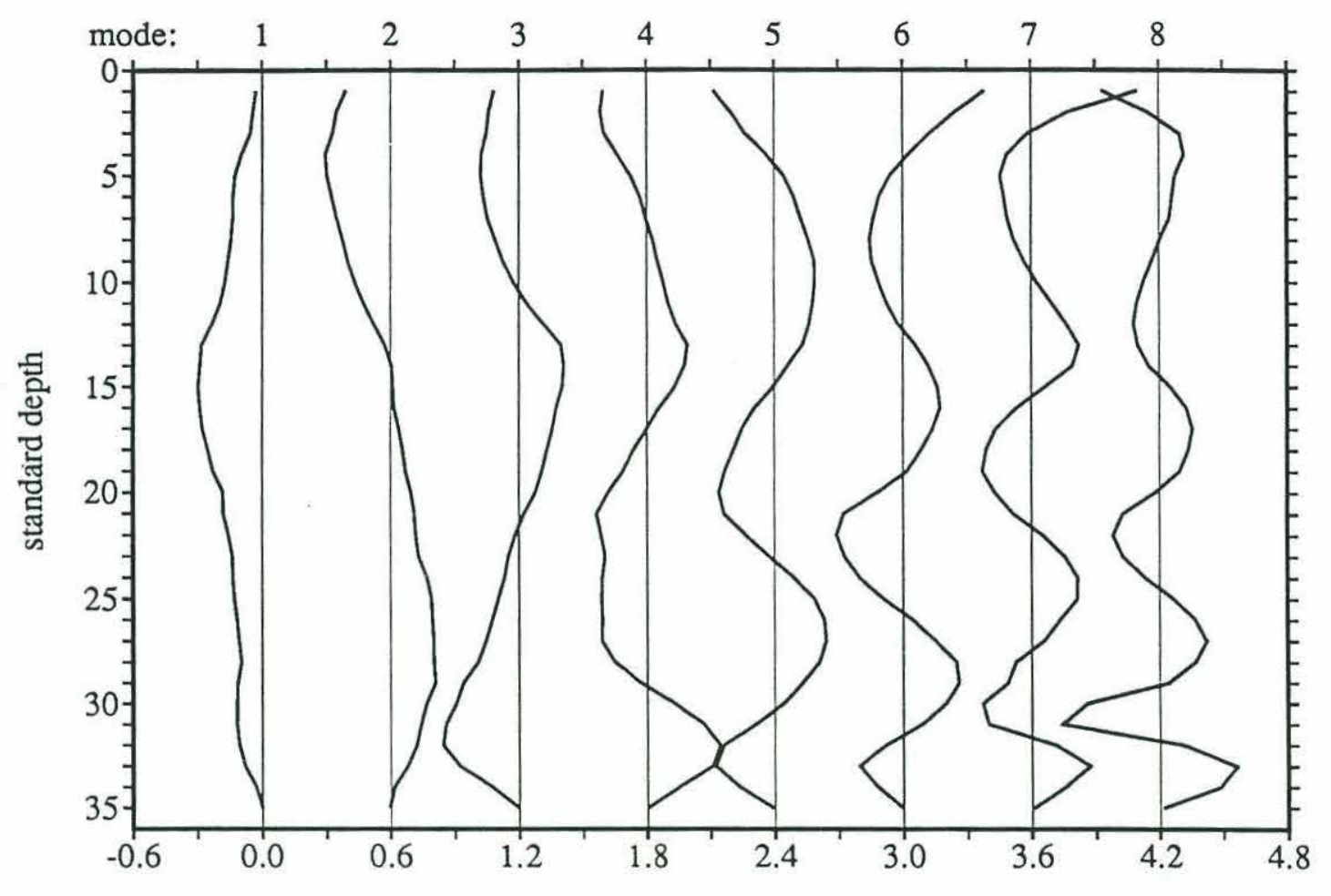

Figure 2-9. Leading 8 Vertical Modes

Higher modes are each offset 0.6 units. (Thin vertical line is the reference line, zero.)

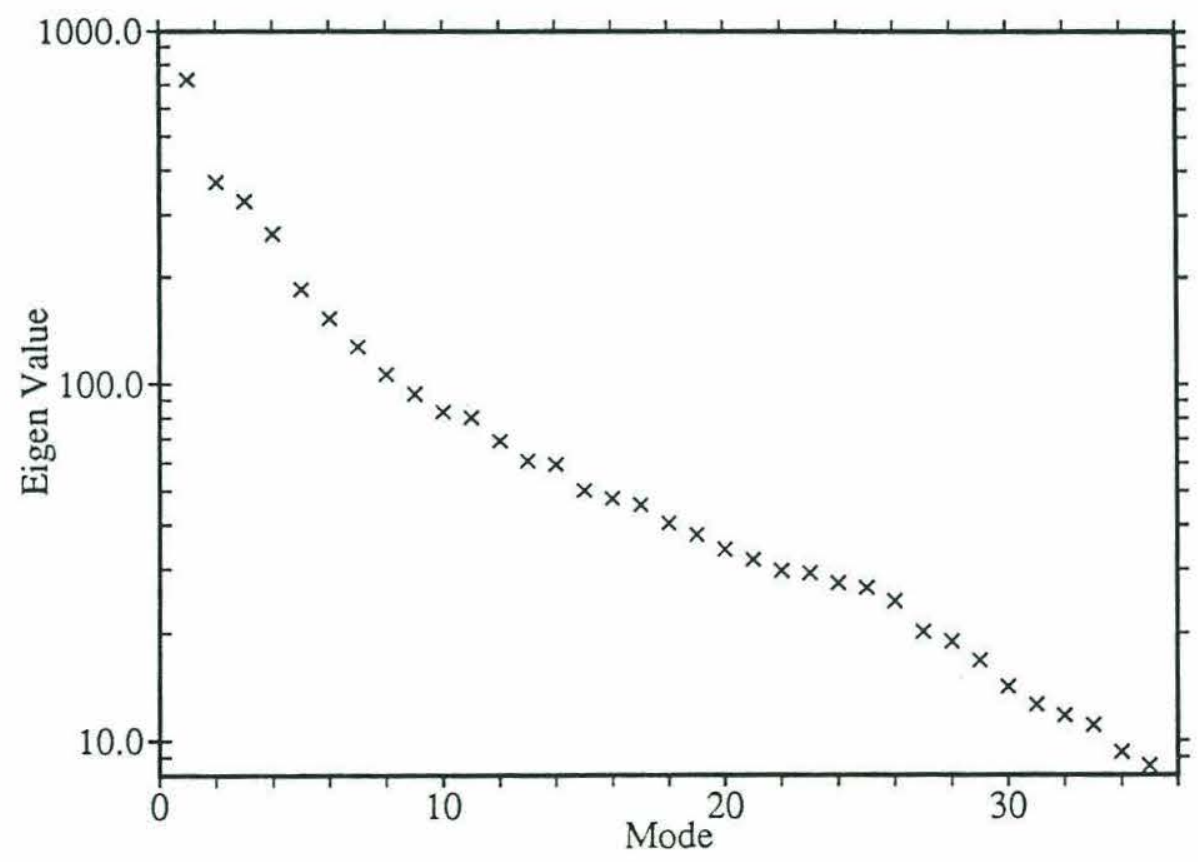

Figure 2-10. Singular Values vs. Mode Number 


\begin{tabular}{|c|c|c|}
\hline mode & $\lambda$ & $\%$ \\
\hline 1 & 721.9 & 53.6 \\
2 & 370.8 & 67.7 \\
3 & 327.0 & 78.7 \\
4 & 264.4 & 85.9 \\
5 & 184.5 & 89.4 \\
6 & 152.7 & 91.8 \\
7 & 127.3 & 93.4 \\
8 & 106.4 & 94.6 \\
9 & 93.8 & 95.5 \\
10 & 83.4 & 96.2 \\
\hline
\end{tabular}

Table 2-2. First Ten Singular Values and Percentage Variances Percentage variance of a particular mode is the percentage of the total data variance the sum from the first mode up to that mode accounts for.

\begin{tabular}{|c|c|c|c|c|c|c|}
\hline modes & $\mathrm{T}$ & $\mathrm{S}$ & $\mathrm{O}$ & $\mathrm{P}$ & $\mathrm{Si}$ & $\mathrm{N}$ \\
\hline 0 & 1.9812 & 2.0622 & 2.3257 & 2.1174 & 2.5254 & 2.3209 \\
1 & 1.5331 & 1.4338 & 1.4601 & 1.4003 & 1.5893 & 1.5223 \\
2 & 1.2763 & 1.2446 & 1.1783 & 1.0923 & 1.2341 & 1.1894 \\
3 & 1.0843 & 1.0027 & 0.9051 & 0.8303 & 1.0003 & 0.9327 \\
4 & 0.8829 & 0.8046 & 0.7422 & 0.6803 & 0.7563 & 0.7703 \\
5 & 0.7342 & 0.6494 & 0.6812 & 0.5955 & 0.6705 & 0.6885 \\
6 & 0.6042 & 0.5676 & 0.6040 & 0.5203 & 0.6060 & 0.5983 \\
7 & 0.5188 & 0.4935 & 0.5428 & 0.4634 & 0.5212 & 0.5298 \\
8 & 0.4629 & 0.4450 & 0.4906 & 0.4294 & 0.4693 & 0.4842 \\
9 & 0.4234 & 0.3999 & 0.4568 & 0.3701 & 0.4146 & 0.4248 \\
10 & 0.3782 & 0.3638 & 0.4333 & 0.3477 & 0.3792 & 0.3989 \\
\hline
\end{tabular}

Table 2-3. RMS Nondimensional Residuals

These are the normalized root mean square residuals of each variable as a function of number of modes retained. ( 0 modes corresponds to the normalized data norm.) 
1). Thus the gravest structural variations are still similar whether in a single section or the whole ocean.

\subsection{Singular Values}

The singular values decrease exponentially with higher modes (Figure 2-10). The magnitudes of the singular values are about 10 times larger than what we had in . Chapter 1, reflecting the order of magnitude more stations we are analyzing for the whole North Atlantic. The percentage variance (Table 2-2) explained as we increase the number of modes displays a similar magnitude as in Chapter $1.85 \%$ of the total variance is already accounted for by the first four modes. Interestingly, the first mode can account for more than half of the total variance whereas the first mode in the one section modal decomposition only explained $38 \%$.

\section{Residuals}

\subsection{Overall Residuals}

Residuals are the differences between original data and modal reconstructions of the data. Table $2-3$ shows that root mean square residuals over all depths and all stations decrease with the number of modes at almost the same rate among separate variables. This near uniform decrease in residual indicates that the modes are indeed identifying common variations among different variables, and not for any one particular property. Comparison with Table 1-3 of the previous chapter shows that the variance of the data (rank 0) is larger for the entire North Atlantic than for one meridional section in the western North Atlantic Ocean (i.e. Endeavor 129). This reflects the greater diversity in hydrographic structure over the entire ocean than over a smaller region. Seven modes are needed for the entire ocean to attain a residual magnitude similar to a four mode reconstruction of the previous chapter.

The structures of the residuals as a function of depth are shown in Figure 2-11 for the first 10 modes. Most depths show a large decrease with the first few modes. 
A. temperature

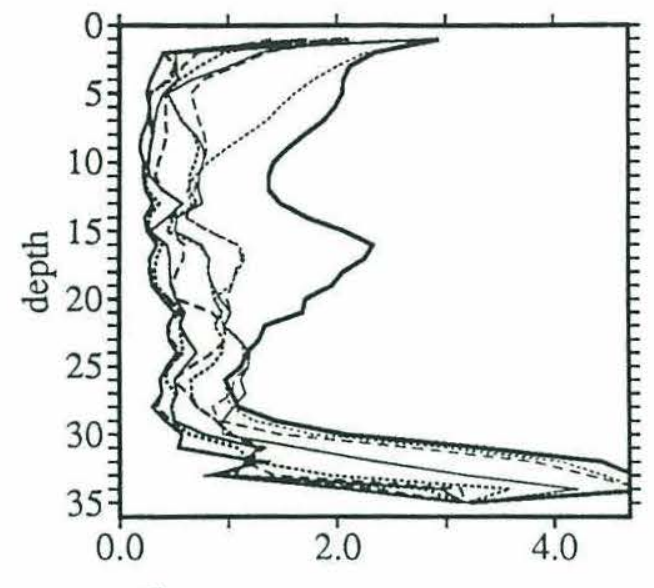

C. oxygen

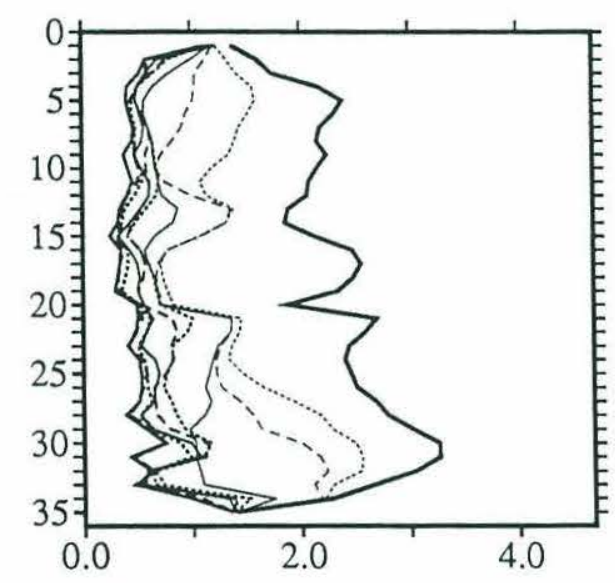

E. silicate

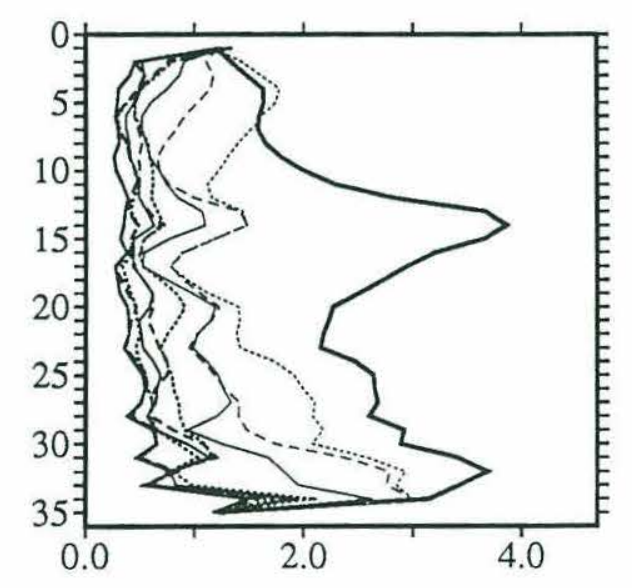

B. salinity

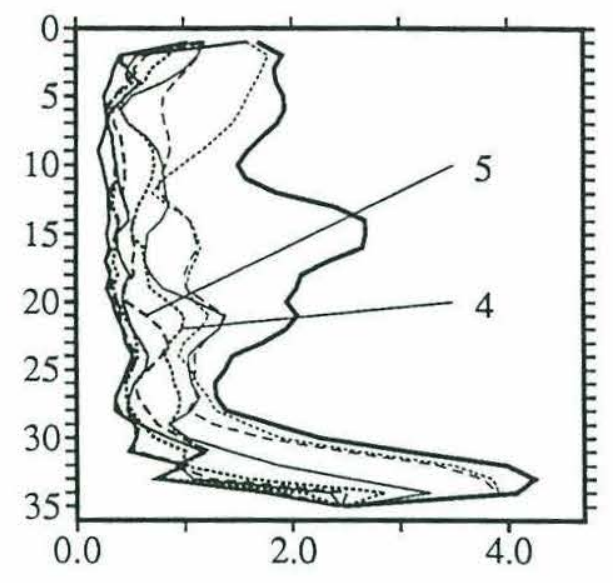

D. phosphate

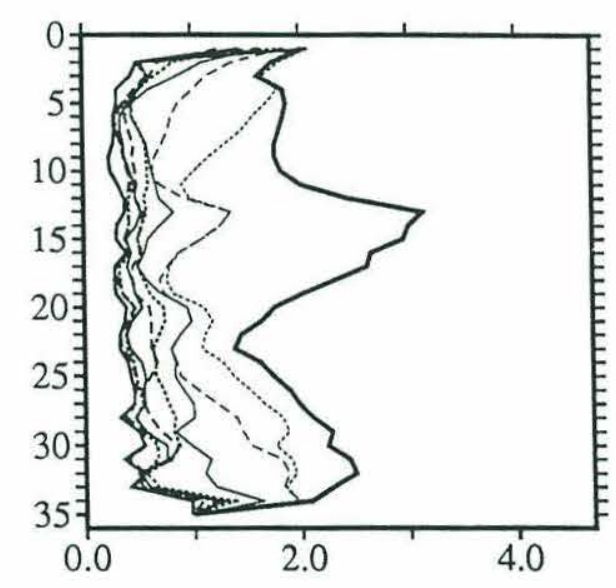

F. nitrate

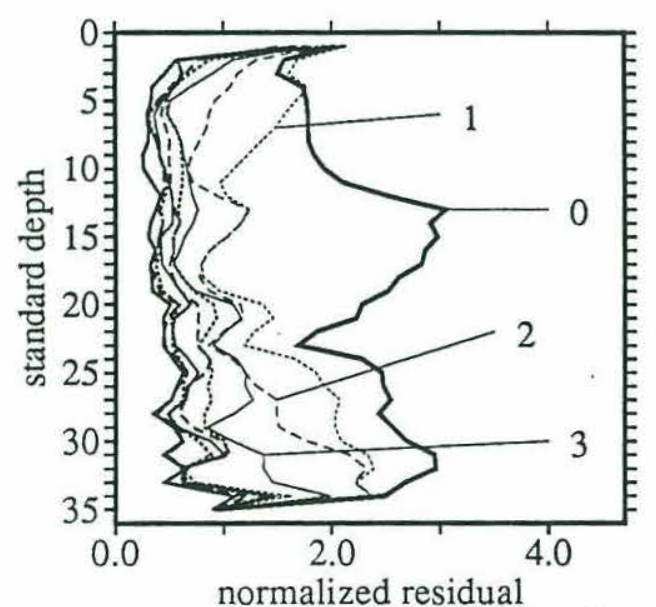

Figure 2-11. (normalized) RMS Residual Vertical Profile Over Entire Ocean Thickest curve is RMS variance of the original data. Each profile represents a different sum of modes. A higher mode truncation is a more dense curve. For example, the thinnest dotted curve is truncated at mode 1, thin dashed at mode 2, etc. (cf F. nitrate). Mode 5 truncation is the medium thick dashed curve. 
However, there is no clear threshold for the division between significant modes and insignificant ones. On the other hand, we can see that by mode 5 , the residuals (in normalized units, Section 3) are well below 1 at most depths. Some exceptions are the larger residuals at depths below standard depth 31, especially for temperature and salinity, and the lack of much residual reduction at the surface.

The surface is as well resolved as any other depth, as we shall see in Section 6, so the lack of residual reduction at the surface implies that surface variations are not well correlated with variations at deeper depths. This result is likely due to surface waters being directly altered by local air-sea interactions, rather than surface waters reflecting underlying hydrographic structures. There are seasonalities in the state of the near surface waters in many parts of the ocean. For example, the presence of a deep surface mixed layer is capped by a seasonal thermocline during the summer in the Sargasso Sea (Schroeder et al., 1959).

Depth 35 also lacks almost any variance reduction. However, it turns out that depth 35 is totally unresolved by the low number of modes being used. We shall come back to the question of resolution in Section 6 .

Although residuals at depths 31 to 33 are about 1 (nondimensional unit), the original data variances are fairly large at these depths, so percentage-wise, the reductions are quite significant. The residuals plotted as a function of station position are helpful in understanding the residuals at these depths. Figure 2-12 is the residual (rank 6) of temperature on standard depth 31 for all stations. There are exceptionally large residuals towards the southern parts of the ocean, especially along Knorr $104\left(35^{\circ} \mathrm{W}\right)$, where the residuals have values as large as 8 in normalized units. These large values are the reason for the overall large RMS residual at this depth. The stations with large residuals correspond to the extremely cold and fresh Antarctic Bottom Water, where water temperature is as cold as $1^{\circ} \mathrm{C}$ as compared to $2.4^{\circ} \mathrm{C}$ immediately to the north at same depth (31). Further examination of such spatial dependence of residual value, 


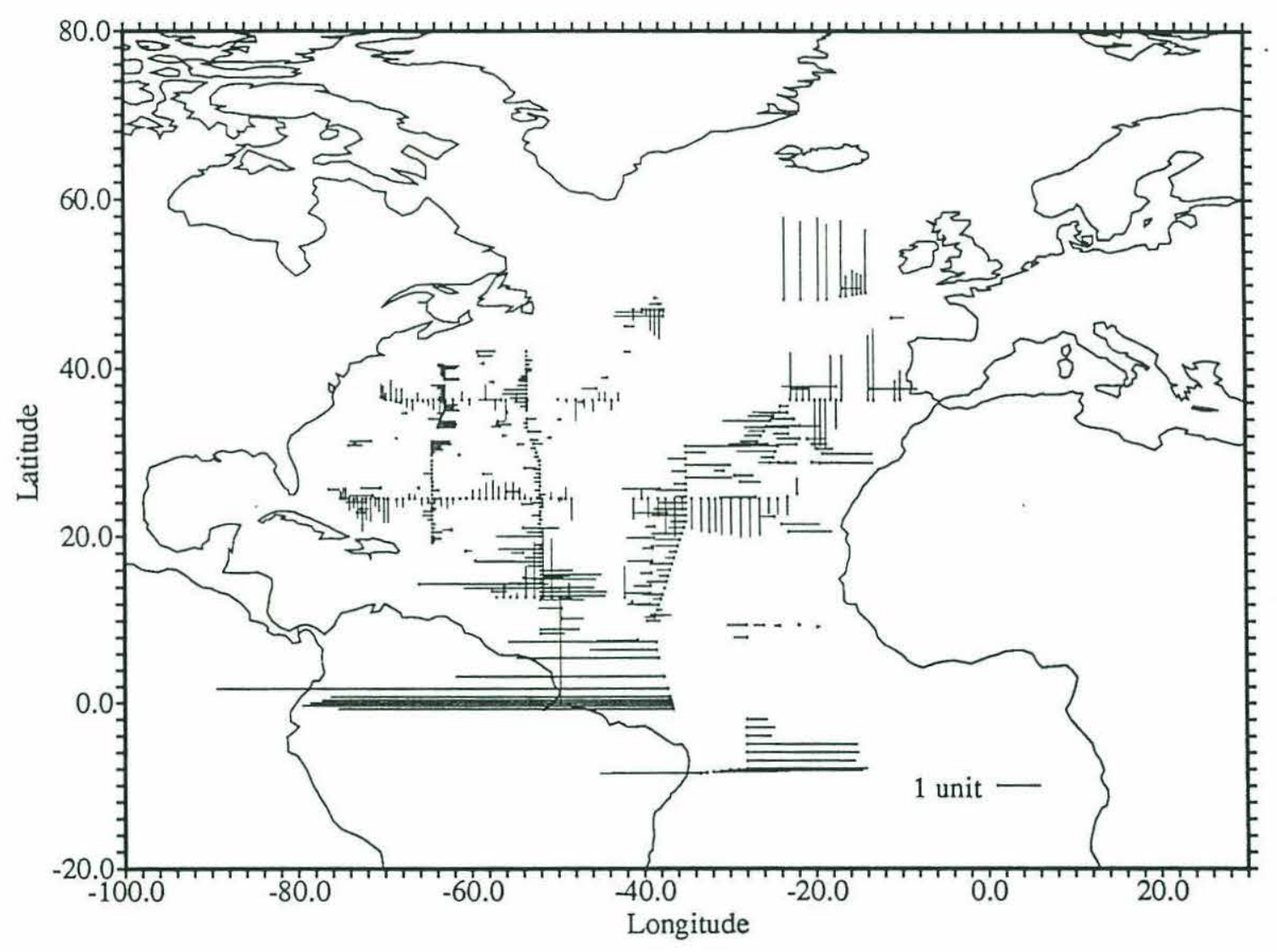

Figure 2-12. Normalized Temperature Residual with 6 EOF's on Depth 31 A unit normalized residual is shown at the lower right. Sticks pointed north or east are positive, meaning data are warmer than modal reconstruction. 
presented in the next section, is helpful in understanding what structure the modes account for.

\subsection{Spatial Variation of Residual}

Figure 2-13 plots the RMS residual of each variable as a function of the number of retained modes for each separate basin (Figure 2-7). As was seen in the overall residuals, each individual variable displays a rapid decrease with the first few modes. By the first 6 modes, all residuals fall below 1, the a priori measure of goodness of fit.

However, one cannot escape noticing a basin dependence in the magnitude of each residual and the rate of decrease with different modes. Basin 3 has the largest residual at mode 6. On the other hand this basin has the largest initial variance, and turns out to have the largest amount of residual reduction. Basin 2 has the smallest residual at mode 6. In most basins the most significant decrease of residual occurs with modes 1 and 2. However, modes 3 and 4 show a significant residual decrease in basin 3 , especially for temperature, whereas the decrease by these modes in other basins are not as dramatic. This different behavior of residual reduction implies that the third and fourth modes may be accounting for hydrographic variations characteristic of basin 3 , but not present in the other five basins.

The relatively large residuals in basin 3 are not due to residuals at all depths, but turn out to be due to residuals isolated in depth. This can be seen in the residual profiles of each property in each basin shown in Figure 2-14, for the sum of the first 6 modes. For basin 3, most parts of the water column have residuals smaller than 1, similar to any of the other 5 basins. However, depths deeper than standard depth 29 have exceedingly large residuals. These large residuals are the same ones identified in Figure 2-12. Namely, the large residuals in basin 3 are primarily due to residuals at bottom depths in the core of Antarctic Bottom Water. 
A. Basin 1

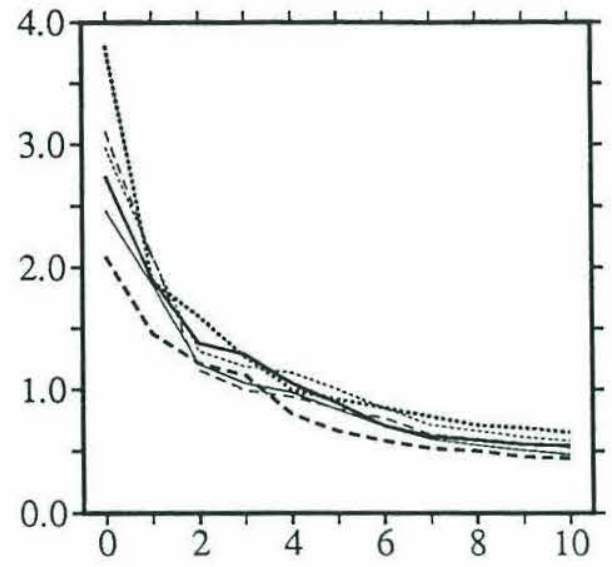

B. Basin 2

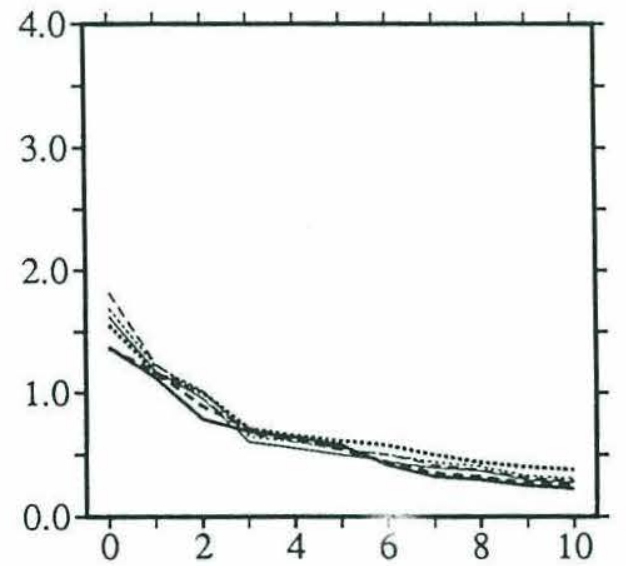

C. Basin 3

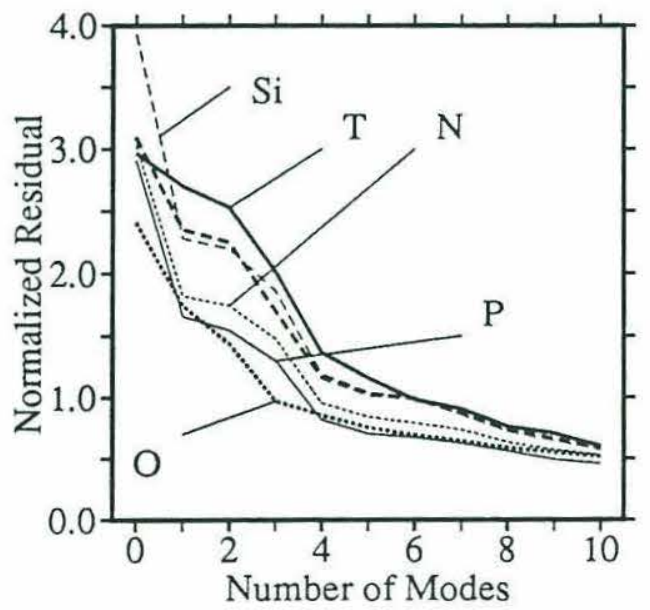

F. Basin 6

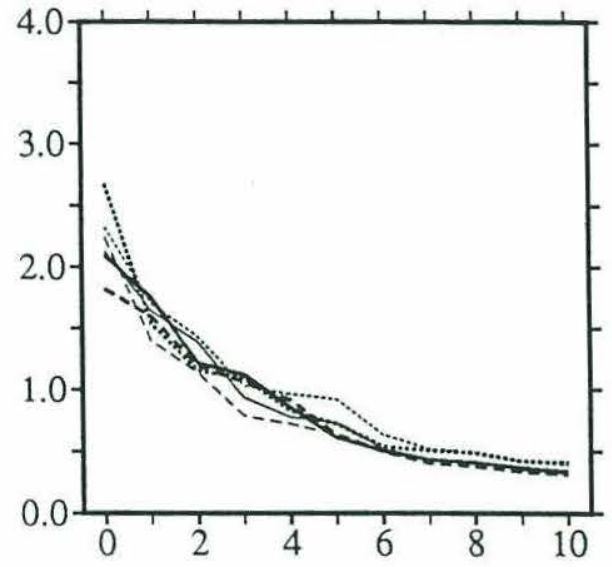

E. Basin 5

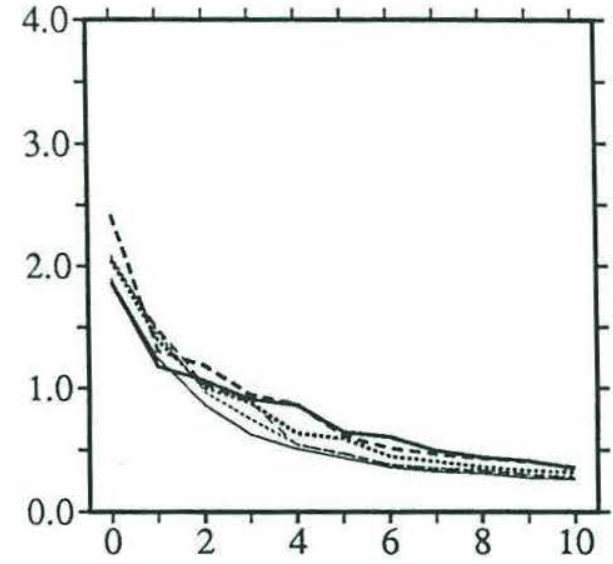

D. Basin 4

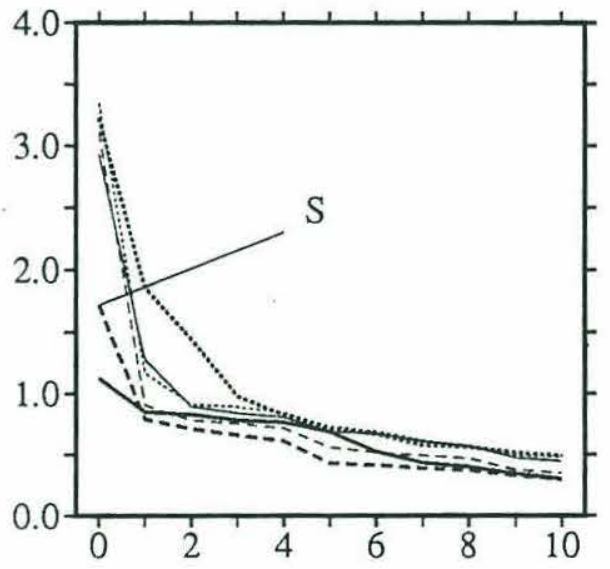

Figure 2-13 RMS Residual vs. Number of Retained Modes in each Basin. A different curve in each plot corresponds to a different property. The thick solid curve is temperature, thick dashed salinity, thick dotted oxygen, thin solid phosphate, thin dashed silicate, and thin dotted is nitrate. 
A. Basin 1

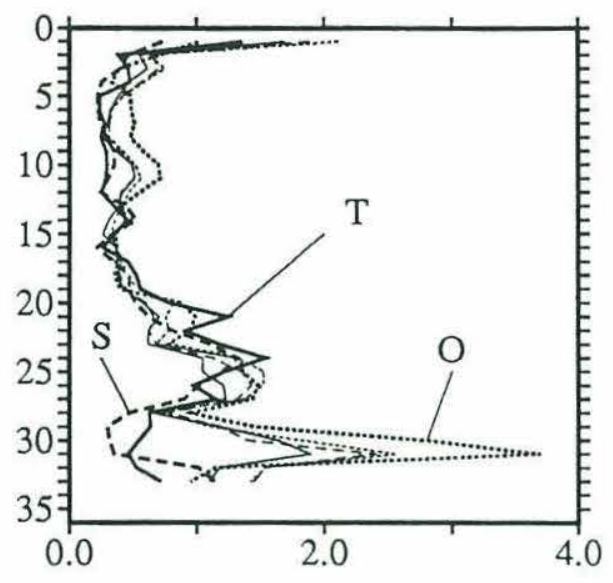

B. Basin 2

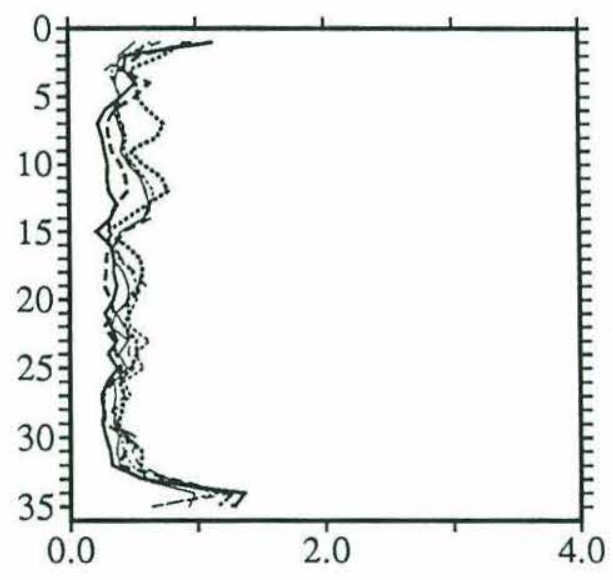

C. Basin 3

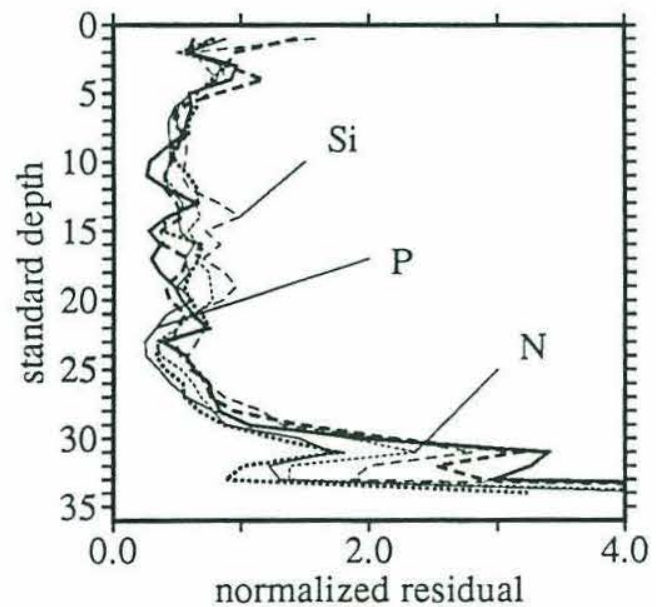

F. Basin 6

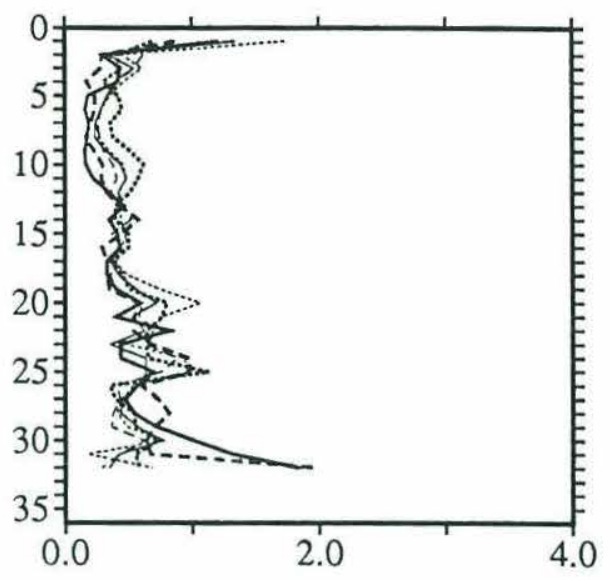

E. Basin 5

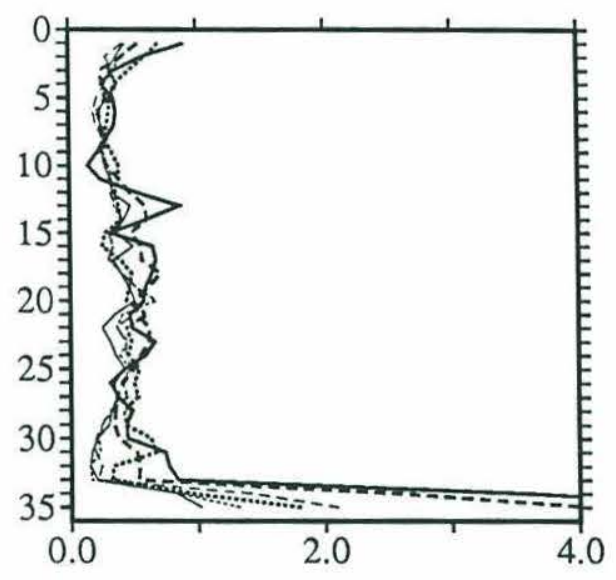

D. Basin 4

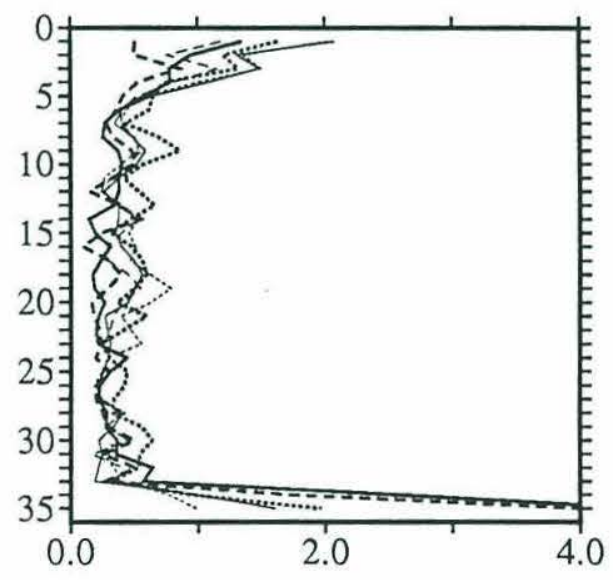

Figure 2-14 RMS Residual Profile Truncated at Mode 6 for each Different Basin. Different curves correspond to different properties as in Figure 2-13. The thick solid curve is temperature, thick dashed salinity, thick dotted oxygen, thin solid phosphate, thin dashed silicate, and thin dotted is nitrate. 


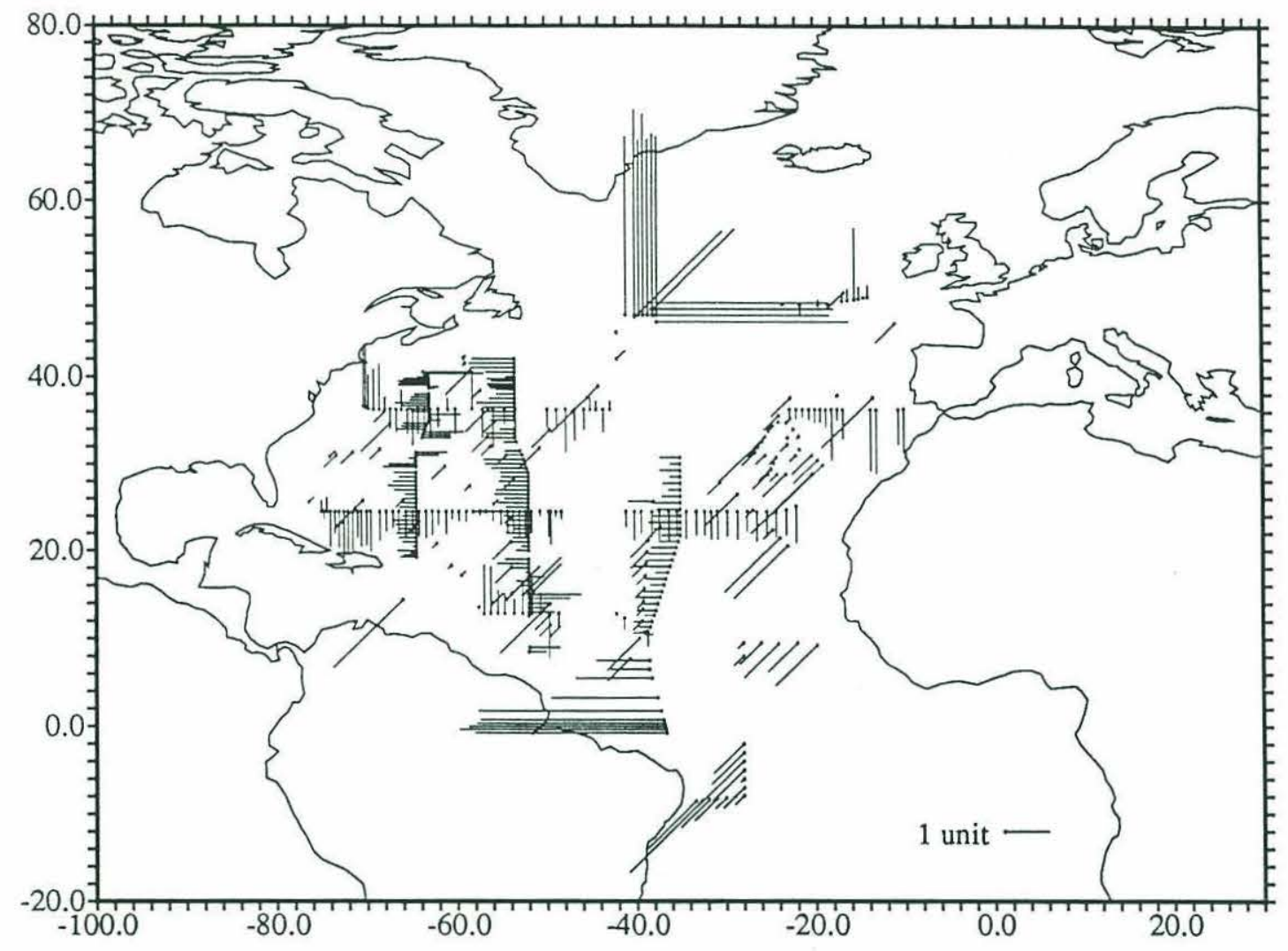

Figure 2-15. Normalized Oxygen Residual with 6 EOF's on Depth 31 A unit residual, which is equal to the normalization factor, is shown at the lower right. Sticks pointed north, east, or northeast are positive, meaning data are richer in oxygen than modal reconstruction. 
Also, there are large residuals in basin 1 below depth 21. These residuals occur at the bottom of the stations or immediately adjacent to continental boundaries, which water are of Norwegian Sea or Labrador Sea origin flowing south as boundary currents. For example, Figure 2-15 plots the residual of oxygen after 6 modes on standard depth 31. This situation is similar to the results in the Antarctic Bottom Water of basin 3. As was the case in basin 3, the original (normalized) data at these depths are anomalously large and although the amount of variance reduction by the modes are larger than other places, the residuals are still one of the largest and the hydrographic structure of these boundary currents are only partially resolved. The primary reason for the large residual is because these boundary currents are not ubiquitous features. We could perhaps account for these better by using a different set of modes. For example, we might calculate modes by using data from basin 1 or basin 3 alone so that the abyssal water masses would be accounted for better.

\subsection{Examples of Residual and Reconstruction Contours}

Figure 2-16 shows two examples of contoured residuals for salinity and silicate, left when using the first six modes. (The percentage variance left in the entire North Atlantic Ocean for salinity and silicate are $7.6 \%$ and $5.8 \%$, respectively (Table 2-3). ) The section is Knorr 104 along $35^{\circ} \mathrm{W}$, which contains the largest hydrographic variation in one section among the sections used in this study. The residuals are in general small in comparison with the variations seen in the original data contour plots (Figure 2-3) and there is little discernible structure left in these residuals, i.e. the residuals are not systematic. However, exceptions are the abyssal waters in the Guiana basin (between $0^{\circ} \mathrm{N}$ and $8^{\circ} \mathrm{N}$ ) and the bottom waters in the Labrador basin (north of $48^{\circ} \mathrm{N}$ ), where the residual magnitudes are much larger than at other stations. These locations are the places we have already identified as having large residuals (Section 5.2). 

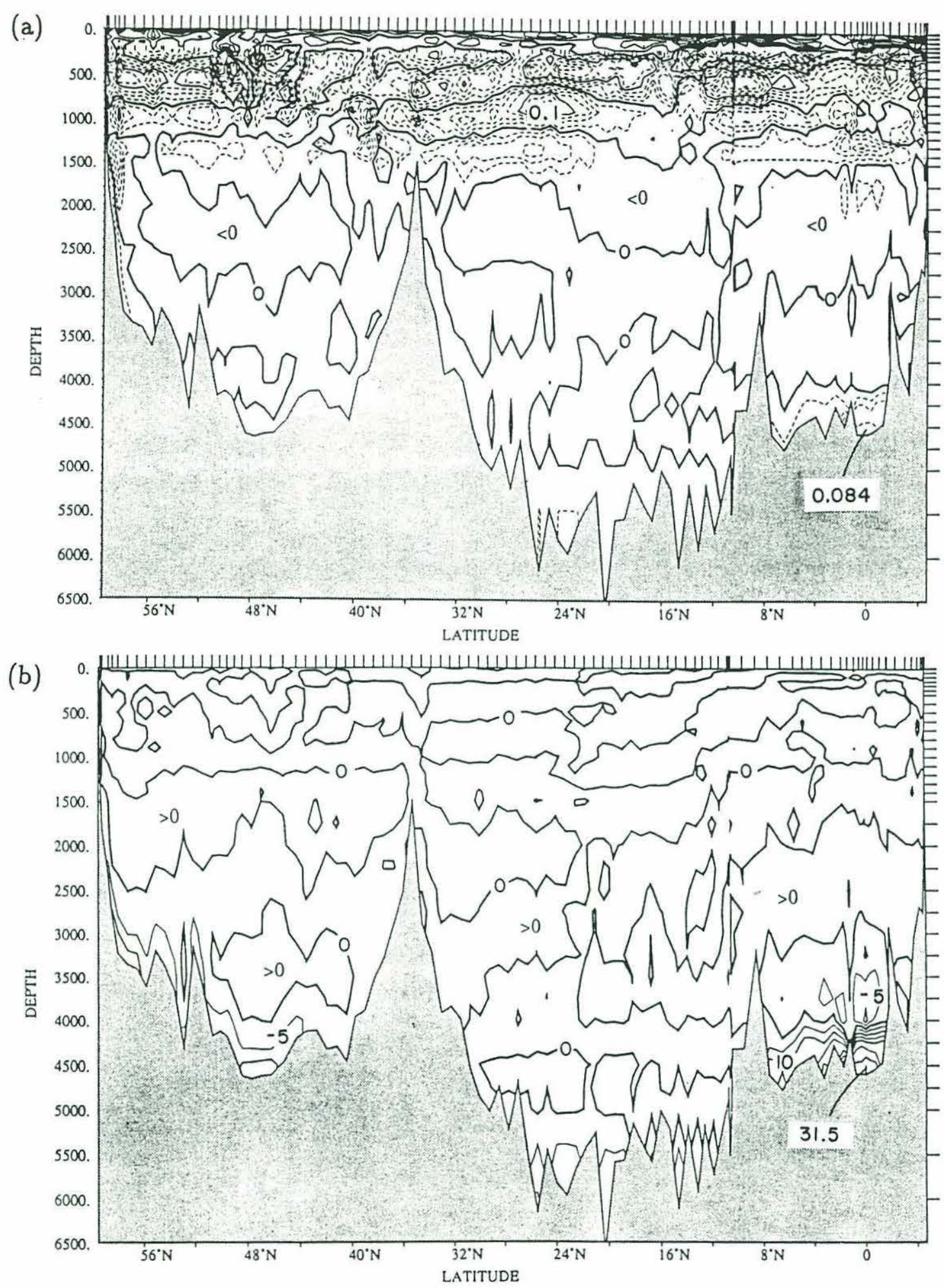

Figure 2-16. Residual Contours at rank 6 along Knorr $104\left(35^{\circ} \mathrm{W}\right)$. (a) Salinity (PSU)

(b) Silicate $(\mu \mathrm{mol} / \mathrm{kg})$

Contour intervals are the same as in Figure 2-3. The thickest solid curve is the zero contour. 


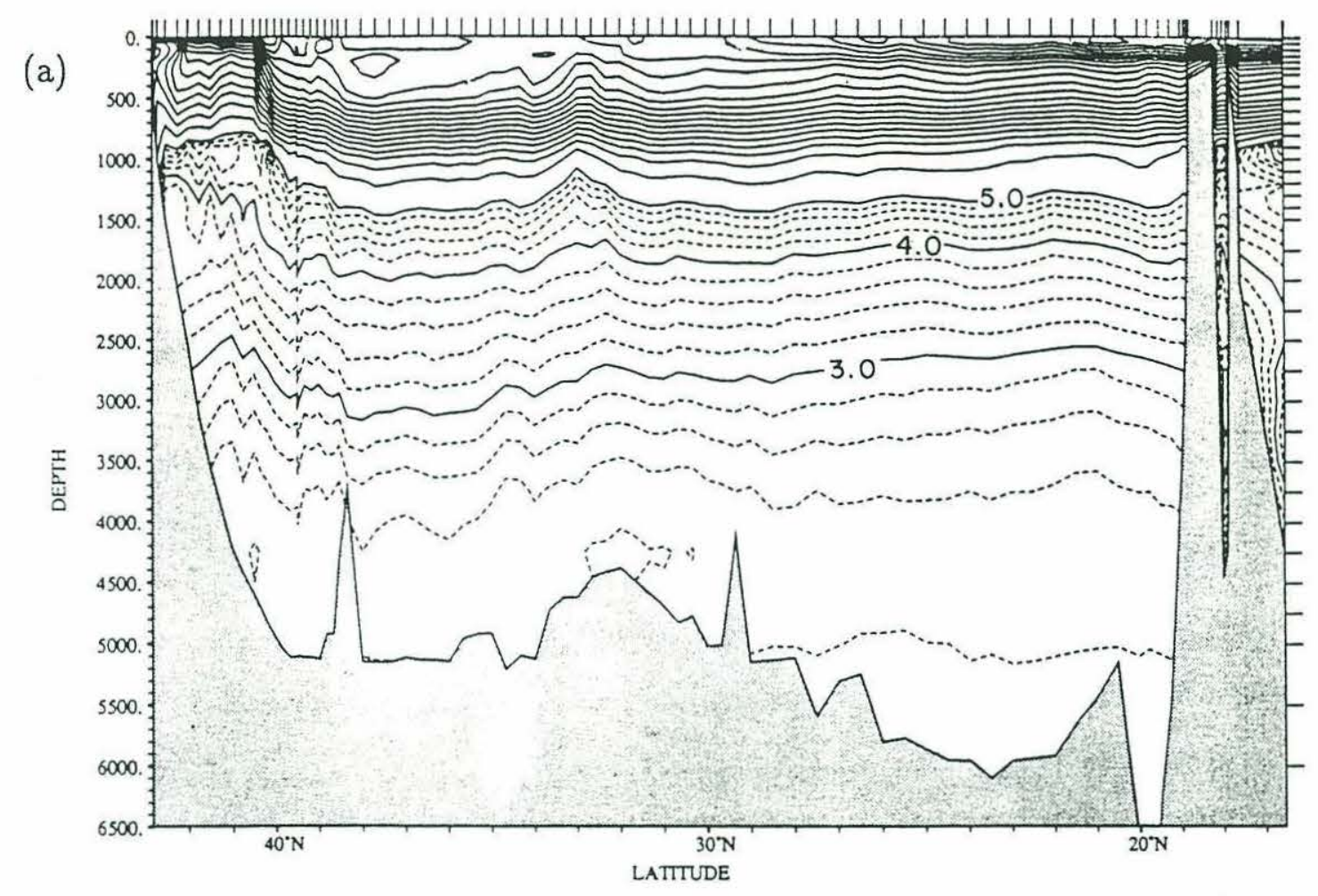

(b)

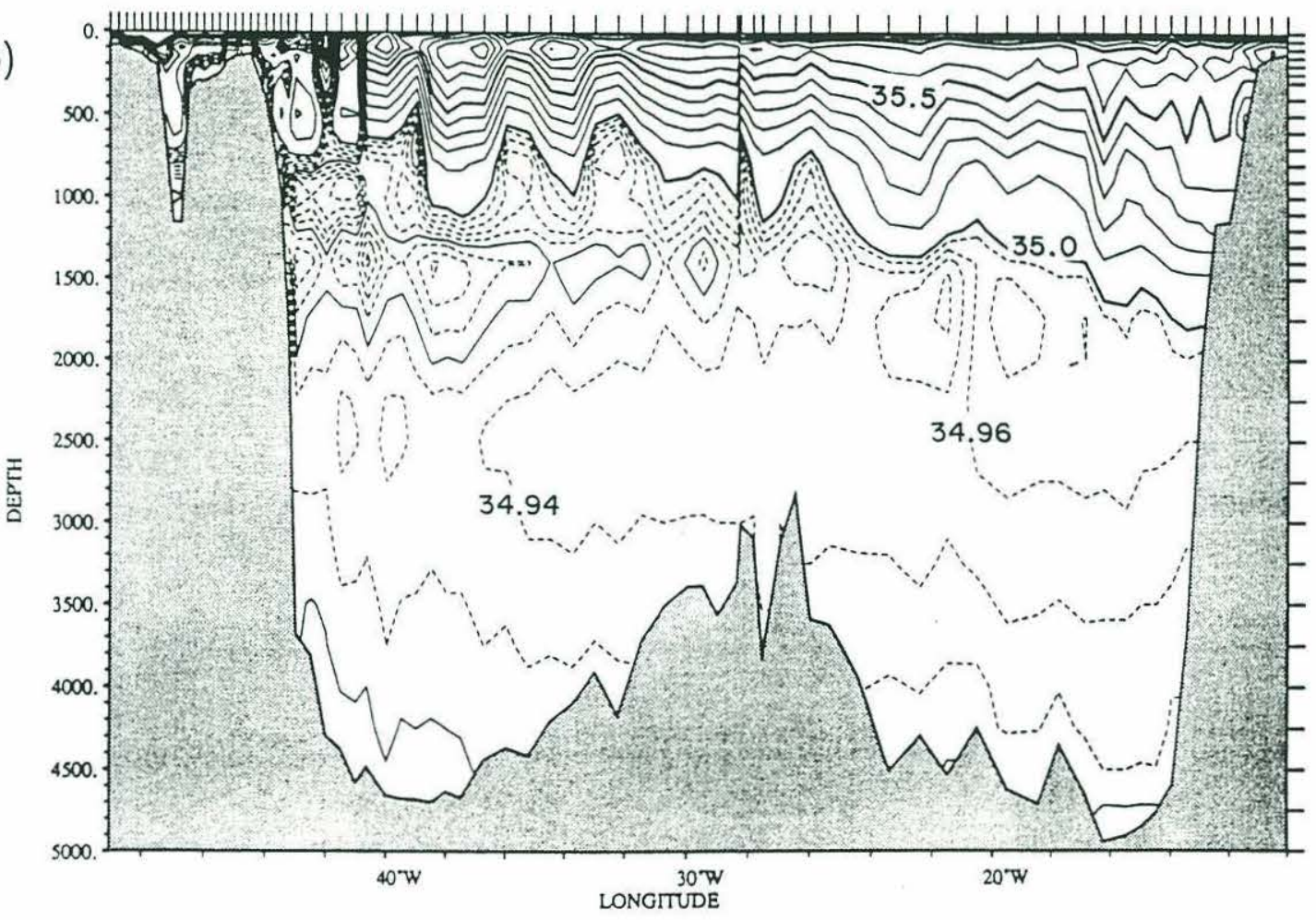

Figure 2-17. 6 Mode Reconstruction of Data

(a) Temperature $\left({ }^{\circ} \mathrm{C}\right)$ - Endeavor $129\left(64^{\circ} \mathrm{W}\right)$ (b) Salinity (PSU) - Hudson $82\left(48^{\circ} \mathrm{N}\right)$ Contour intervals are the same as in the original data contours: e.g. Figures 1-2 and $2-4$. 

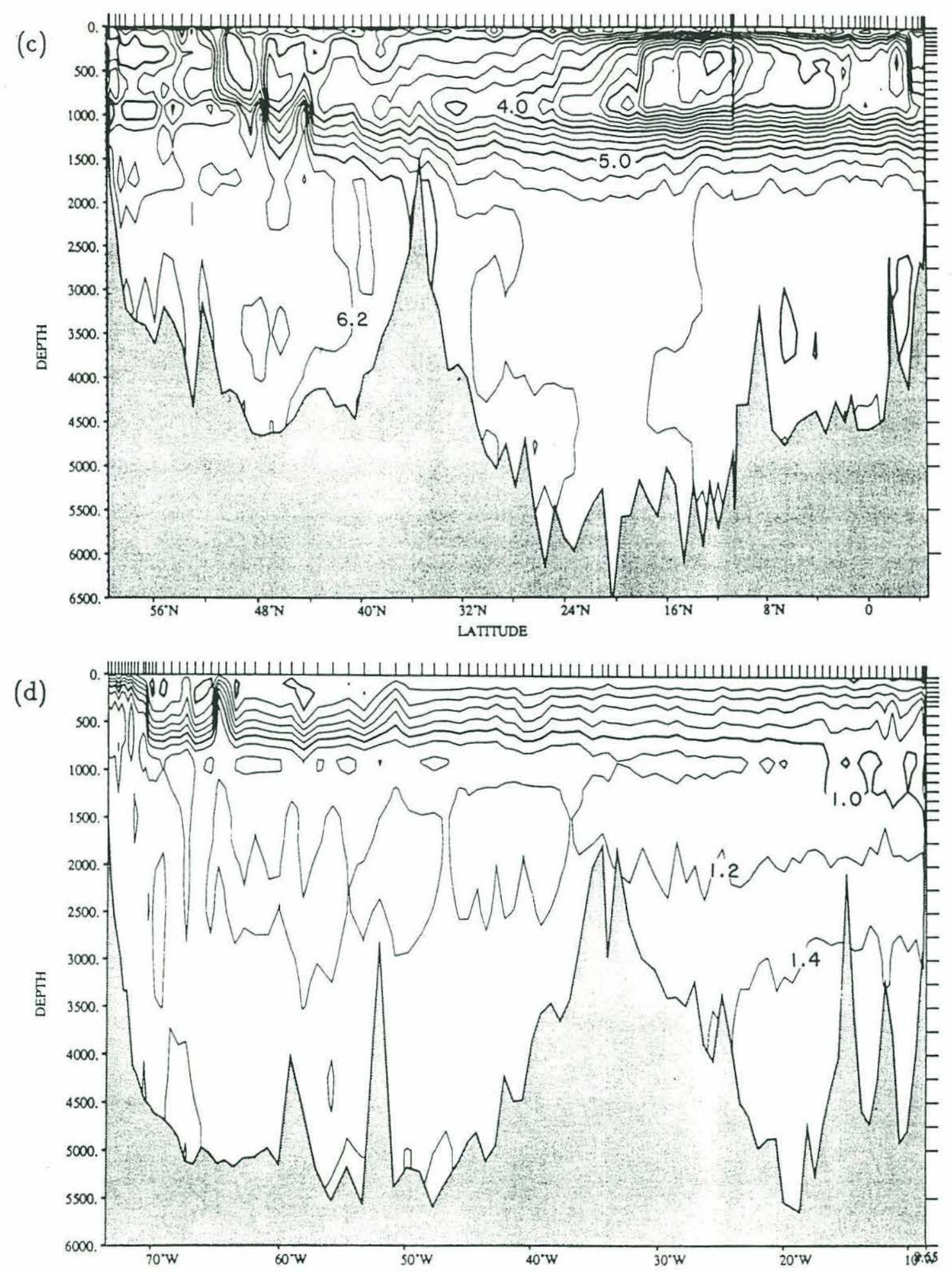

Figure 2-17. (continued) 6 Mode Reconstruction of Data

(c) Oxygen $(\mathrm{ml} / \mathrm{l})-$ Knorr $104\left(35^{\circ} \mathrm{W}\right)$

(d) Phosphate $(\mu \mathrm{mol} / \mathrm{kg})-$ Atlantis II $109 \operatorname{leg} 1\left(36^{\circ} \mathrm{N}\right)$ 


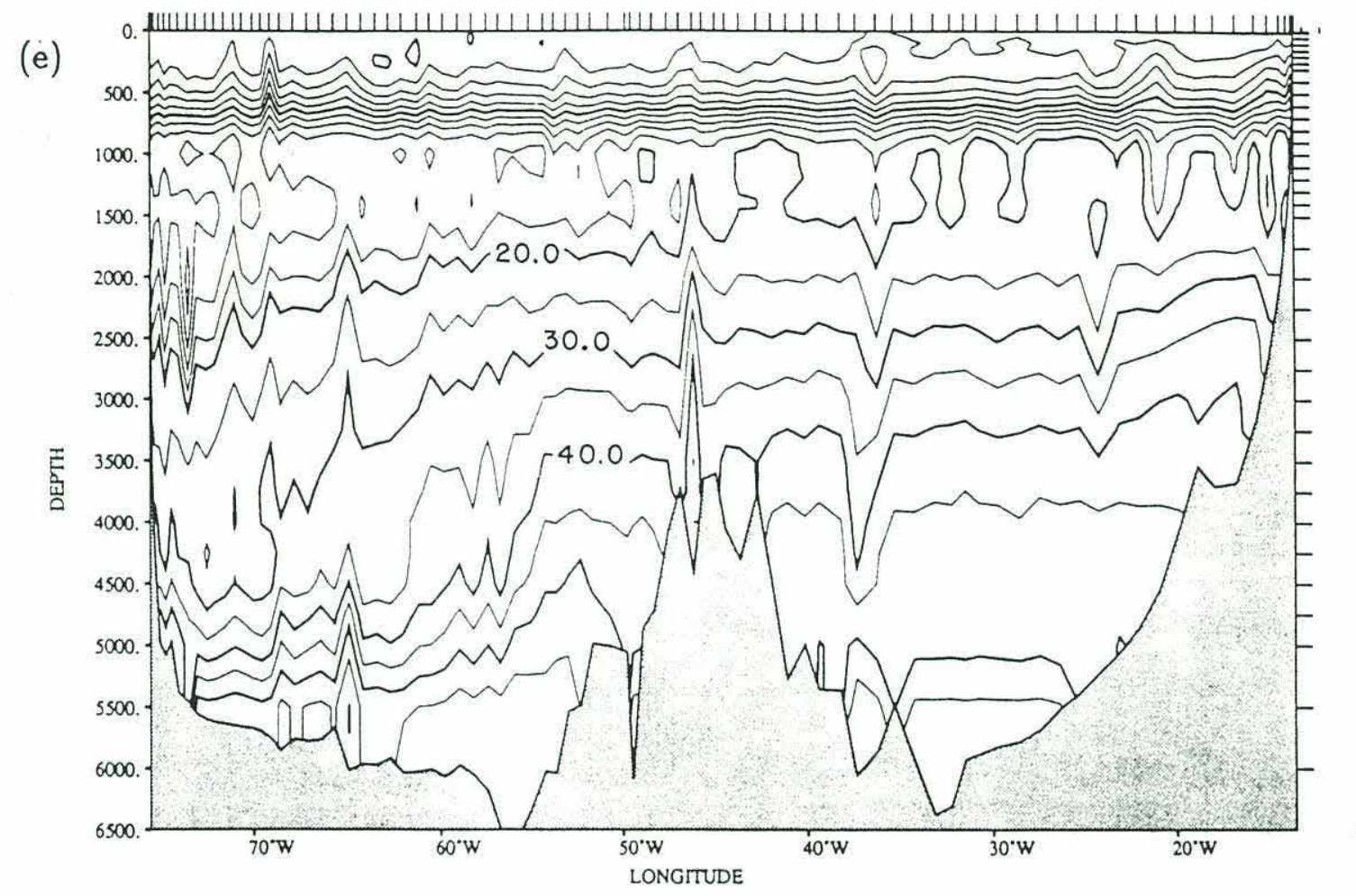

(f)

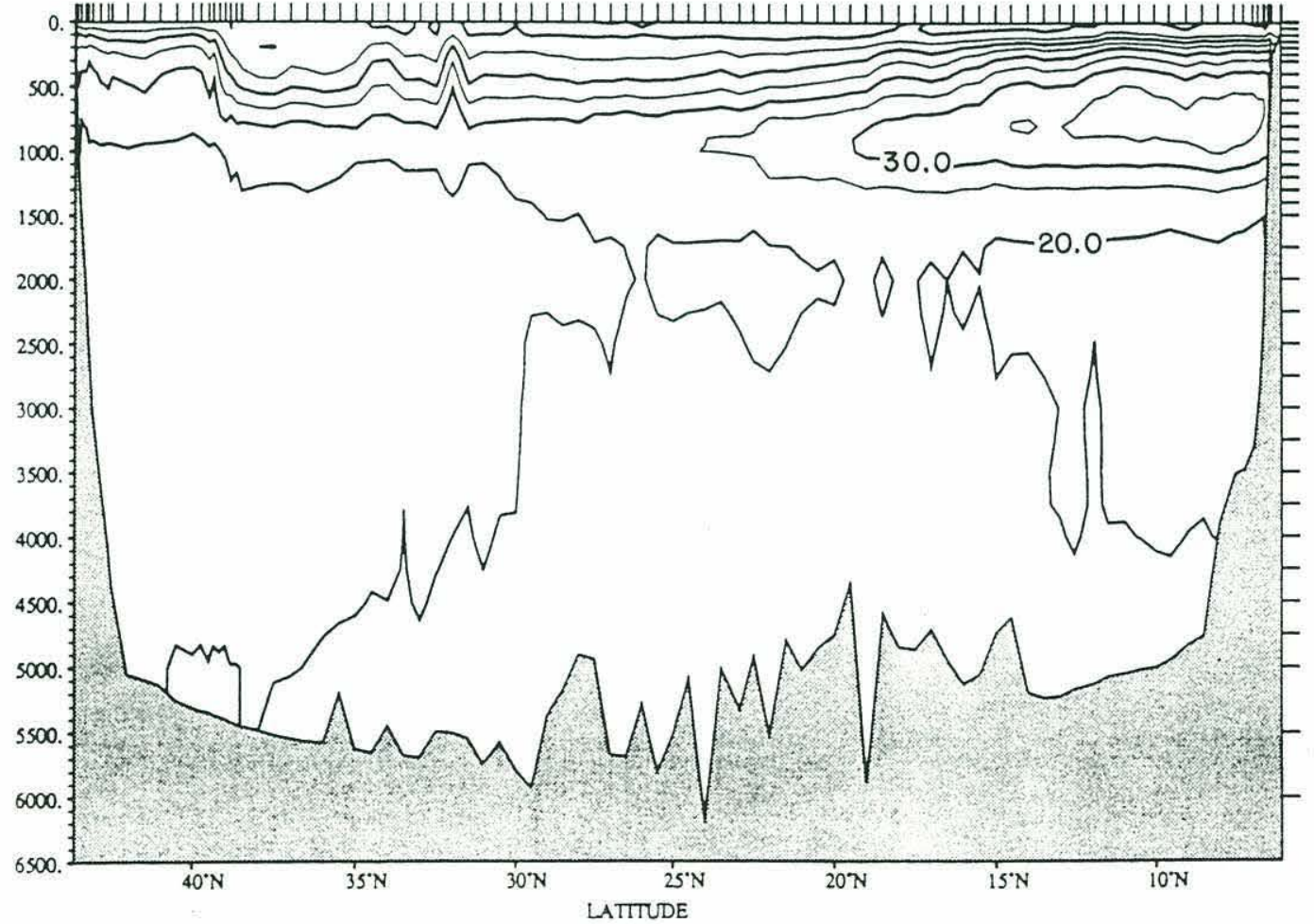

Figure 2-17. (continued) 6 Mode Reconstruction of Data

(e) Silicate $(\mu \mathrm{mol} / \mathrm{kg})$ - Atlantis II 109 leg $3\left(24^{\circ} \mathrm{N}\right)$

(f) Nitrate $(\mu \mathrm{mol} / \mathrm{kg})$ - Oceanus 133 leg $7\left(53^{\circ} \mathrm{W}\right)$ 
Figure 2-17 shows some examples of the reconstructed fields by sums of the first six modes. Here we present contour plots of six different properties, each along a different hydrographic section. To the eye, the major hydrographic structures of the original data are well reproduced in all six contour plots.

\subsection{Summary}

To summarize, with as many as the first 6 modes, the residuals are small enough in most parts of the ocean to be considered insignificant. There are some limited regions where these residuals are larger than other areas, and larger than the a priori measure of significance. These residuals elucidate regional physical differences and in turn point to regions of interest. The regions include the surface waters, waters inside the core of the Antarctic Bottom Water spreading north at bottom depths in the western equatorial regions, and waters of Labrador Sea-Greenland Sea origin spreading south as a deep western boundary current in the northwestern region.

\section{Resolution}

An ocean that can be modeled by a few modes imply that the overall ocean structure is simple. This also means that a variation at one depth is well correlated to variations at other depths. However, caution must be exercised in the interpretation of such a model with low modes, because the high correlation will in turn mean the model will not account well for variations that have small depth scales. Such short scale features inevitably do exist at least in some parts of the ocean. The problem is of resolution.

The normalized data, $\underline{t}_{d a t a}$ (variation from the mean profile normalized by significance estimate), is modeled by a sum of modes, $\underline{t}_{\text {modal }}$;

$$
\underline{t}_{\text {data }} \approx \sum_{i}^{6} \underline{u}_{i} \alpha_{i}=U \underline{\alpha}=\underline{t}_{\text {modal }} .
$$


The coefficients, $\alpha_{i}$, are obtained by,

$$
\underline{\alpha}=U^{T} \underline{t}_{d a t a} .
$$

So, $\underline{t}_{\text {modal }}$ is related to the real data by,

$$
\underline{t}_{\text {modal }}=U \underline{\alpha}=U U^{T} \underline{t}_{d a t a} .
$$

Thus, we see that the model is really a filtered version of the data, the filter being the resolution matrix, $U U^{T}$. The resolution matrix tells us which combination of the data is resolved. This situation is equivalent to the data resolution matrix of an inverse problem (e.g. Wunsch, 1978).

Figure 2-18 shows the resolution matrix using the first 6 modes. The elements of the 35th row and 35th column are virtually zero, which means depth 35 is not accounted for at all by the first 6 modes. The lack of resolution at depth 35 could have been anticipated from the structure of the modes; namely, the elements of the 35th depth are essentially zero for any of the leading EOF's (Figure 2-9). The absence of variance reduction at this deepest depth (Figure 2-11) is partly a result of this lack of resolution. The unresolvability of depth 35 means that the EOF analysis could not establish a correlation between variations at this depth to other depths. One of the main reasons is likely because there are not as many data points at this depth as the other depths to allow this depth's resolution.

The first row, on the other hand, has similar structure as any of the other rows, even though data reduction was small at depth 1 (Figure 2-11). The large residual at depth 1 would then mean that there are more variations at depth 1 than what meager resolution 6 modes can account for, whereas there are not as much variance left at deeper levels at the same number of modes. This mismatch of resolution and residual is consistent with the notion that the surface has little correlation with structure at 


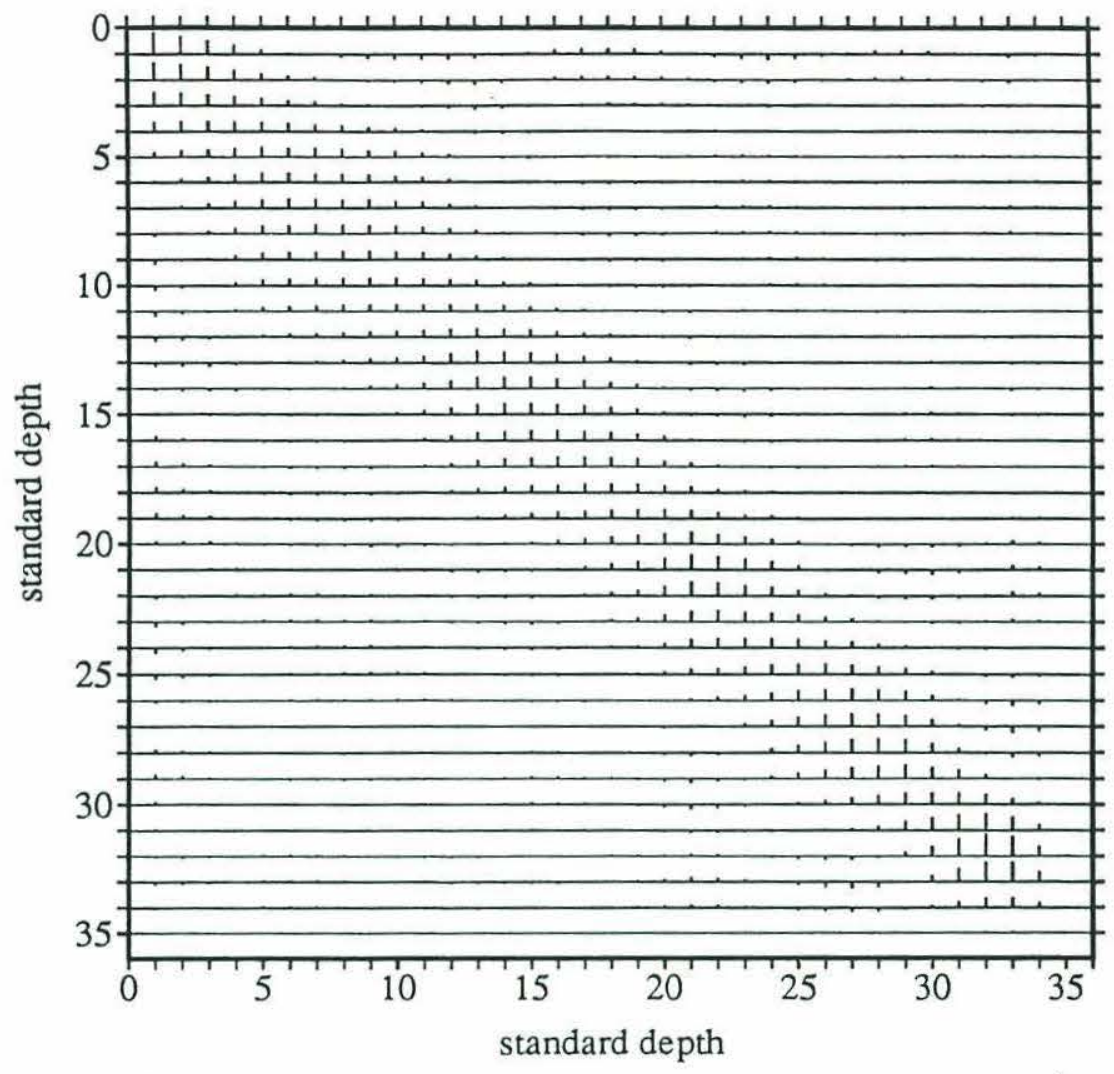

Figure 2-18. Resolution Matrix with 6 EOF's

The scale is such that 2.5 units in the vertical equals 1 in resolution.

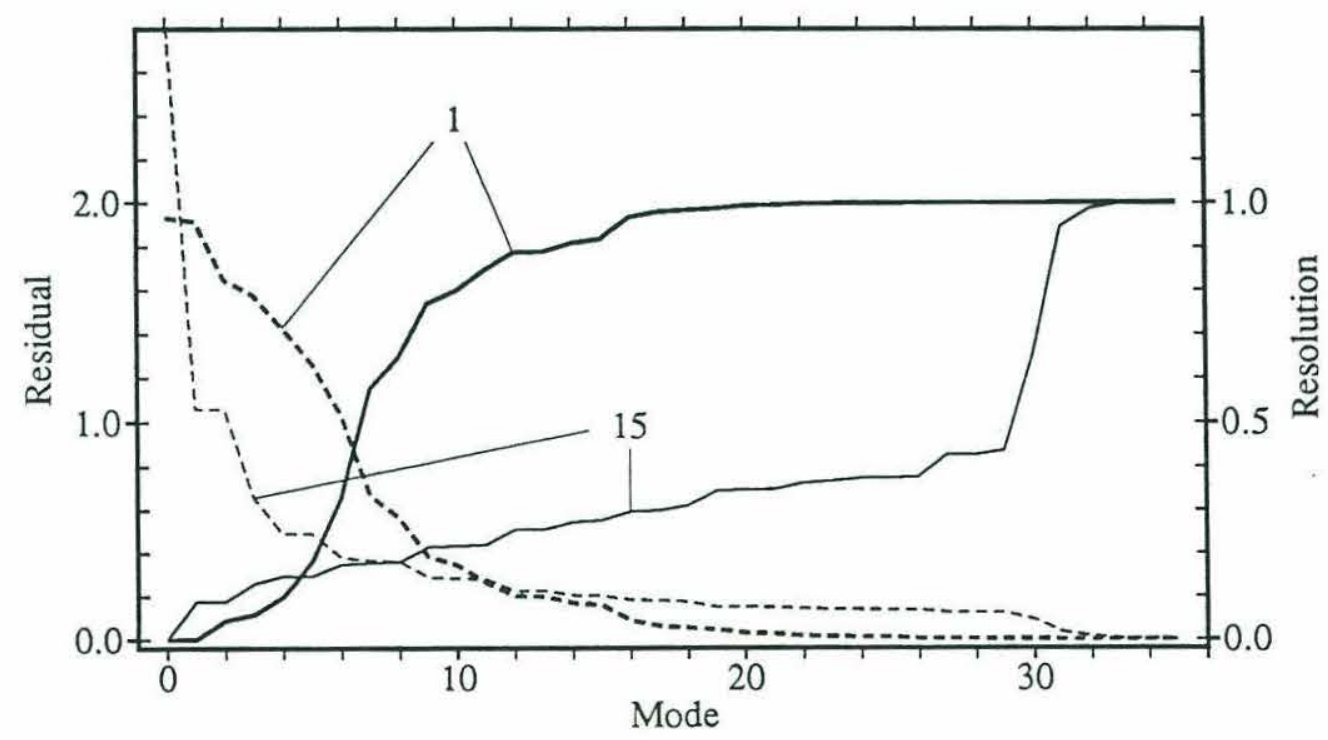

Figure 2-19. Resolution and Residual vs. Number of Modes This compares the increase of resolution and decrease of residual as a function of the number of modes used. The solid curve is resolution and dashed curve is residual. The thicker curve is at depth 1 and the thinner curve is at depth 15 . 
depth, as was mentioned in Section 5. The resolution and total squared residual at depth $i$ for the sum of $k$ modes can be written as,

$$
\begin{aligned}
\text { resolution } & =\sum_{j=1}^{k} U_{i j}^{2}, \\
\text { residual } & =\sum_{j=k+1}^{35} \lambda_{j}^{2} U_{i j}^{2} .
\end{aligned}
$$

$U_{i j}$ is the element of mode $j$ at depth $i$ and $\lambda_{j}$ is the singular value for mode $j$. Although the higher the rank the higher the resolution and smaller the residual, a similar resolution at one depth and at another depth does not guarantee similar residuals at those two depths. Figure 2-19 plots the resolution and RMS residual as a function of the number of modes at standard depths 1 (0 dbar) and 15 (1100 dbar). With 6 modes, the residual of depth 15 is smaller than the residual at depth 1, despite the fact that depth 15 has lower resolution and larger initial (0 mode) data variance.

The diagonal of the resolution matrix has a considerable spread. This implies that no depth is resolved independently from another. This lack of exact resolution can lead to a situation similar to the Gibbs phenomena in spectral analysis (e.g. Bracewell, 1978), which is a large residual (between the original data and a filtered reproduction of it) due to high frequency structure present in the data that the filter can not resolve. Figure 2-20 shows an example of this Gibbs-like phenomena. The salinity profile reconstructed by 6 modes (dashed) has an inversion between $900 \mathrm{~m}$ (13) and $1800 \mathrm{~m}$ (20), which is not present in the data (solid). This is the result of the small vertical scale salinity anomaly between depths 21 to 23 , which shows up in the normalized data. The wide diagonal width of the resolution matrix means that one depth cannot be examined independently from another. To overcome this problem, we could apply a vertical smoothing over a finite depth as a remedy and smooth out possible short-scale artificial variations. An example is shown as the dotted curve in Figure 2-20. A box 


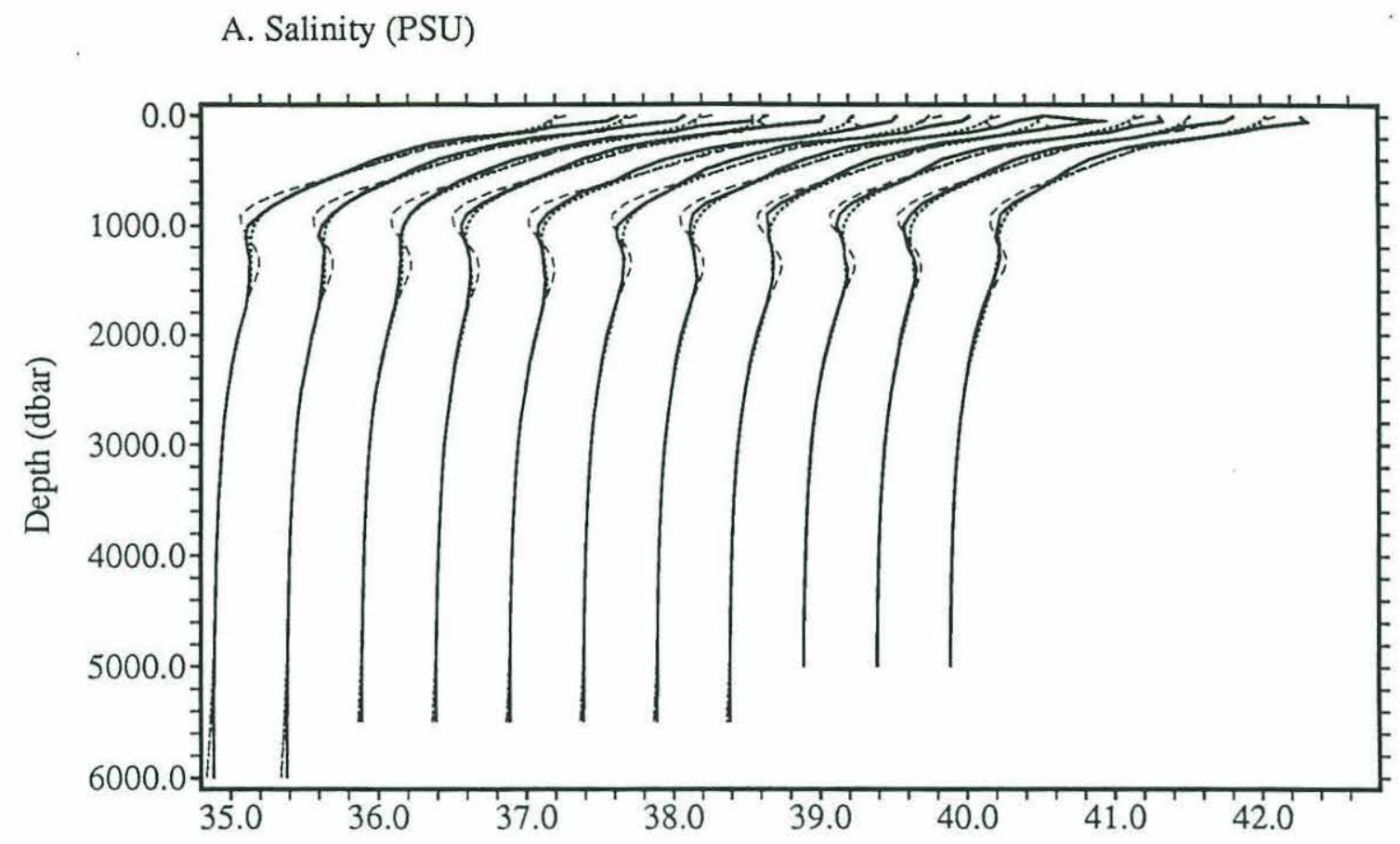

B. Normalized Salinity Profile

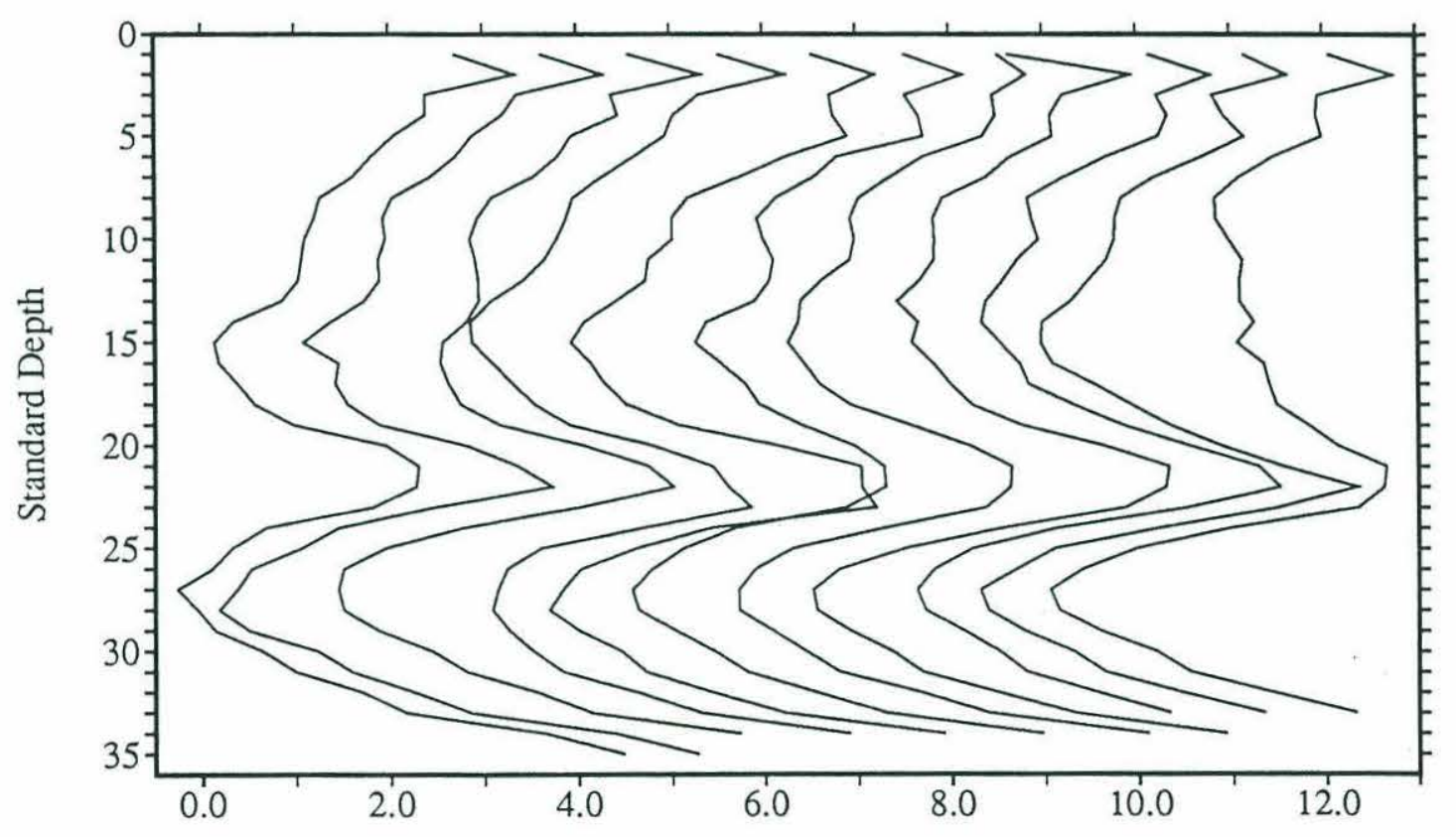

Figure 2-20. Examples of Gibbs-like Phenomena.

The profiles are salinity along Atlantis II 109 leg $3\left(24^{\circ} \mathrm{N}\right)$ from $33.2^{\circ} \mathrm{W}$ to $23.2^{\circ} \mathrm{W}$. The curves in A. are original data (solid), 6 mode reconstruction (dashed), and vertically smoothed 6 mode reconstruction (dotted). Profiles in B. are normalized data. 


\begin{tabular}{|c|c|}
\hline standard depth & smoothing window \\
\hline 1 & $1 \sim 3$ \\
2 & $1 \sim 4$ \\
3 to 6 & 5 standard depths \\
7 to 18 & 7 standard depths \\
19 to 30 & 5 standard depths \\
31 to 33 & 3 standard depths \\
34 to 35 & no smoothing \\
\hline
\end{tabular}

Table 2-4. Smoothing Window

Smoothing is done by a simple box car average over a prescribed window. The windows given above are either the depth limit over which averaging is performed or its width in terms of standard depths centered at the depth of concern.

car average was used to smooth over the reconstructed dimensional profile. The width of the average in terms of standard depths is given in Table 2-4.

\section{Mass Flux}

\subsection{Comparison along Each Section}

In the previous sections, we have seen that the first 6 modes can account for much of the dominant hydrographic structural variations of the North Atlantic Ocean. Here, we examine how well the circulation is accounted for by these modes. In doing so, we compare geostrophic mass transport of data and modal reconstruction along each separate hydrographic section. Geostrophic velocity is calculated relative to $2000 \mathrm{dbar}$ (standard depth 21). As in Chapter 1, this uniform reference level will suffice because our interest is only in a comparison of the general character of the circulation between the original data and the modal reconstruction, rather than a calculation of the absolute circulation. The comparison is made for the 6 mode reconstruction, where 
temperature and salinity are vertically smoothed, as described in the previous section, before computing density.

\section{A. Hudson $82-48^{\circ} \mathrm{N}$}

This section is the northern most east-west one along nominally $48^{\circ} \mathrm{N}$. A comparison of the zonally integrated mass transport profile between data and the 6 mode reconstruction is given in Figure 2-21. Mass flux is calculated between layers, which boundaries are defined as mid depths between the standard depths. To the eye, the data and the modal reconstruction are almost indistinguishable.

The shaded region in Figure 2-21 shows a measure of goodness of fit about the data profile. This measure of error is calculated similar to the way we obtained the measure for normalization in Section 3. First, a measure of dynamic height error (relative to 2000 dbar) was calculated on each depth as the RMS difference between adjacent stations along the trans-ocean sections. Regions about the Gulf Stream and the North Atlantic Current were not included in this calculation. The overall mean distance at one station separation is $65 \mathrm{~km}$. The calculated dynamic height error profile is shown in Figure 2-22. We may interpret this measure as the dynamic height perturbation associated with short scale $(\sim 65 \mathrm{~km})$ eddies which are inherently transient.

Geostrophic velocity, $v$, is written in terms of dynamic height as,

$$
v=\frac{1}{f} \frac{\partial D}{\partial x}
$$

Mass flux along an east-west section ( $f=$ constant) within a layer of fixed thickness $h$ is,

$$
M=\int v h d x=\frac{h}{f}\left(D_{1}-D_{2}\right)
$$

and is only a function of the dynamic heights of the end stations, $D_{1}, D_{2}$. Assuming the error of the end stations to be uncorrelated, the mass flux error can be estimated 


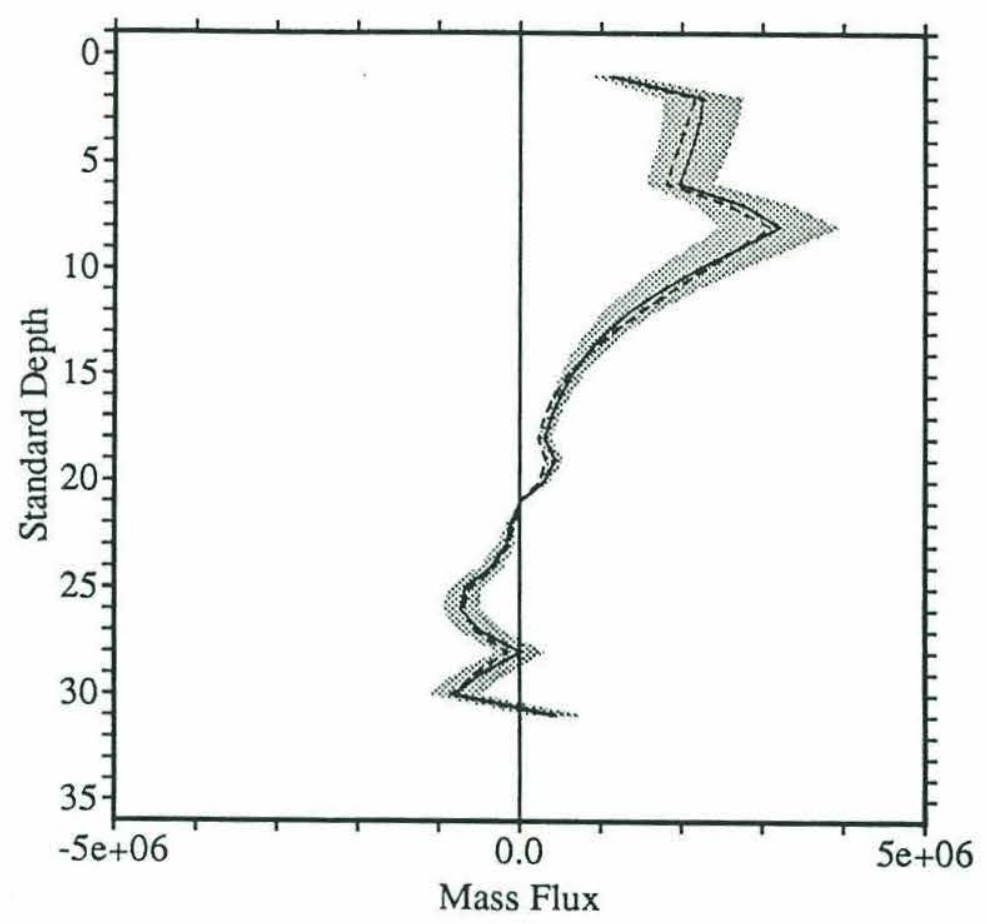

Figure 2-21. Mass Flux $\left(\mathrm{m}^{3} / \mathrm{s}\right)$ Profile of Hudson 82

Solid curve is mass flux of the original data, and dashed curve is that of the 6 mode reconstruction. Shaded region is the original data plus/minus the error estimate. Positive mass flux corresponds to northward flux. See text for details on how mass flux is calculated.

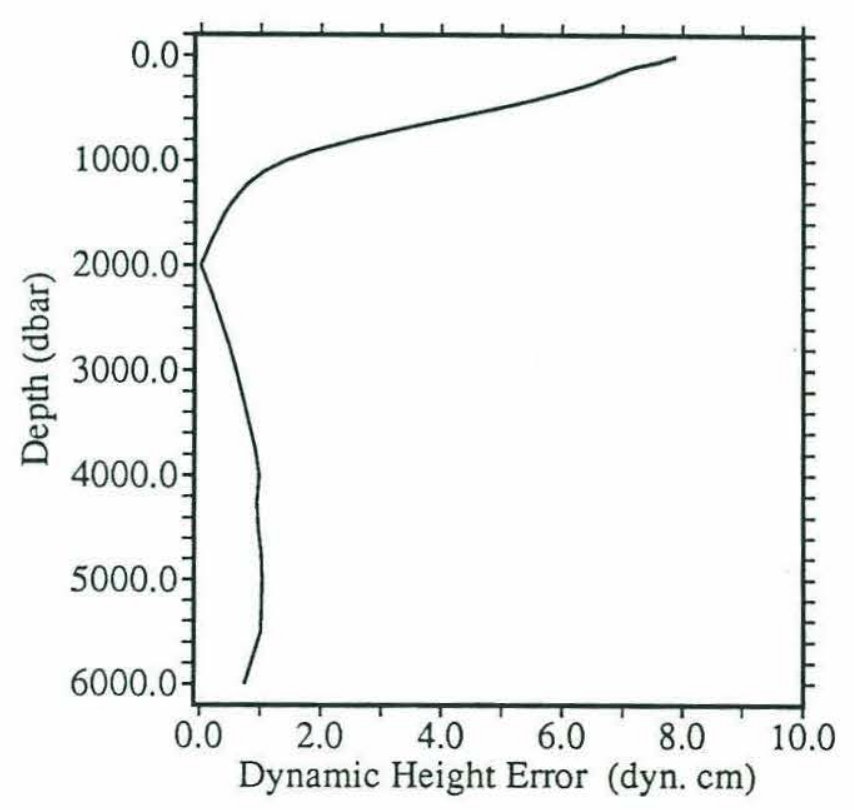

Figure 2-22. Dynamic Height Error Estimate 
by,

$$
\delta M=\frac{h}{f} \sqrt{2} \delta D,
$$

where $\delta D$ is dynamic height error at individual stations (Figure 2-22). $\delta M$ for Hudson 82 is given by the shaded region in Figure 2-21. The modal reconstruction is well within this error bound at all depths.

\section{B. Atlantis II 109 leg $1-36^{\circ} \mathrm{N}$}

The mass flux profile between $73.3^{\circ} \mathrm{W}$ and $8.9^{\circ} \mathrm{W}$ is shown in Figure 2-23a. Although the model is consistent with data at most depths, there appears to be a significant difference (larger than the error bar) between 1100 dbar (standard depth 15) and 1500 dbar (19). To determine why this discrepancy occurs, we examine dynamic height and mass flux at 1300 dbar (17) in Figure 2-24. Modal dynamic height appears to diverge from the data east of about $20^{\circ} \mathrm{W}$. Transports between individual stations also have a larger difference at these longitudes. The region east of $18^{\circ} \mathrm{W}$ is where the influence of the Mediterranean outflow becomes most intense, as seen clearly in either temperature or salinity contour plots, Figure 2-5; the appearance of the 36.0 PSU isohaline about $1200 \mathrm{dbar}$ or the deepening of the $10^{\circ} \mathrm{C}$ isotherm and the lack of temperature stratification about 1200 dbar. For comparison, Figure 2-23b shows the total mass flux profile between $73.3^{\circ} \mathrm{W}$ and $20.6^{\circ} \mathrm{W}$. The agreement between modal reconstruction and data are much better. (The region examined in the inverse problem of Chapter 3 is west of $20^{\circ} \mathrm{W}$.)

Thus, there apparently is some bias in mass flux about the core of the Mediterranean outflow just west of the strait of Gibraltar. However, it is interesting to note that the modal mass flux at shallower levels are in agreement with the data throughout the hydrographic section. We will discuss this point of near surface circulation in more detail in Section 7.2. 
a. $8.9^{\circ} \mathrm{W}$ to $73.3^{\circ} \mathrm{W}$

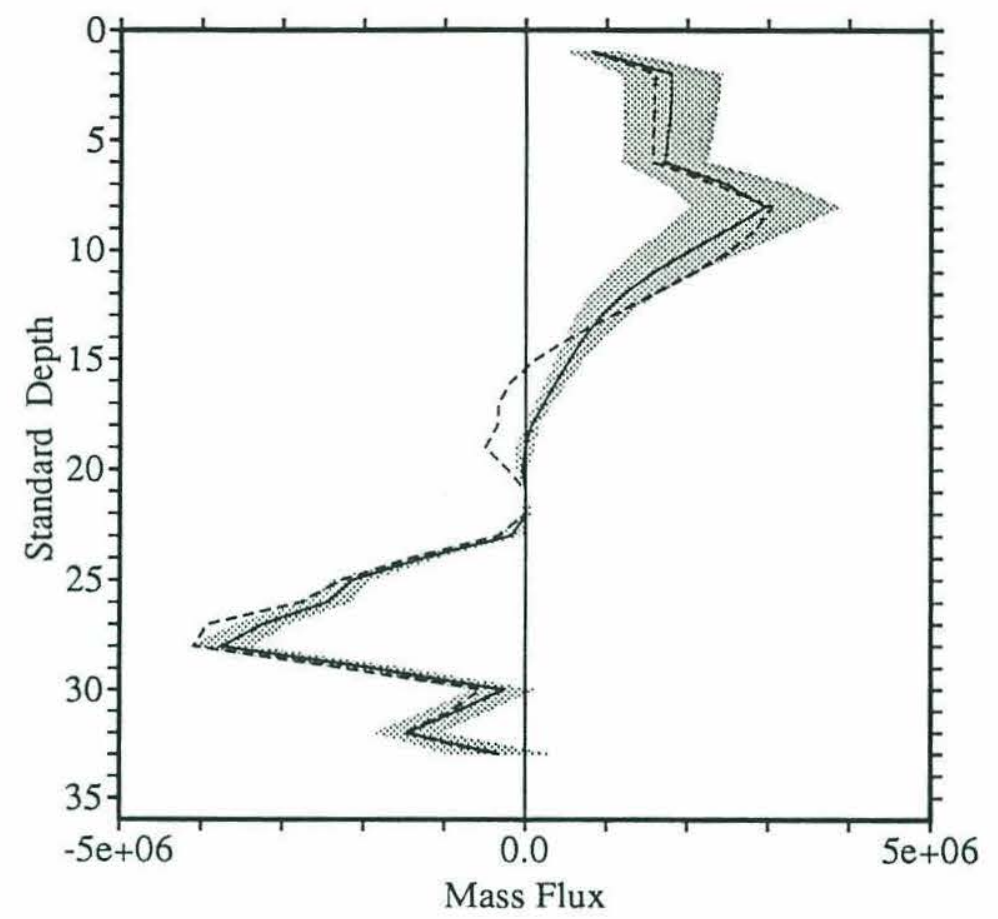

b. $20.6^{\circ} \mathrm{W}$ to $73.3^{\circ} \mathrm{W}$

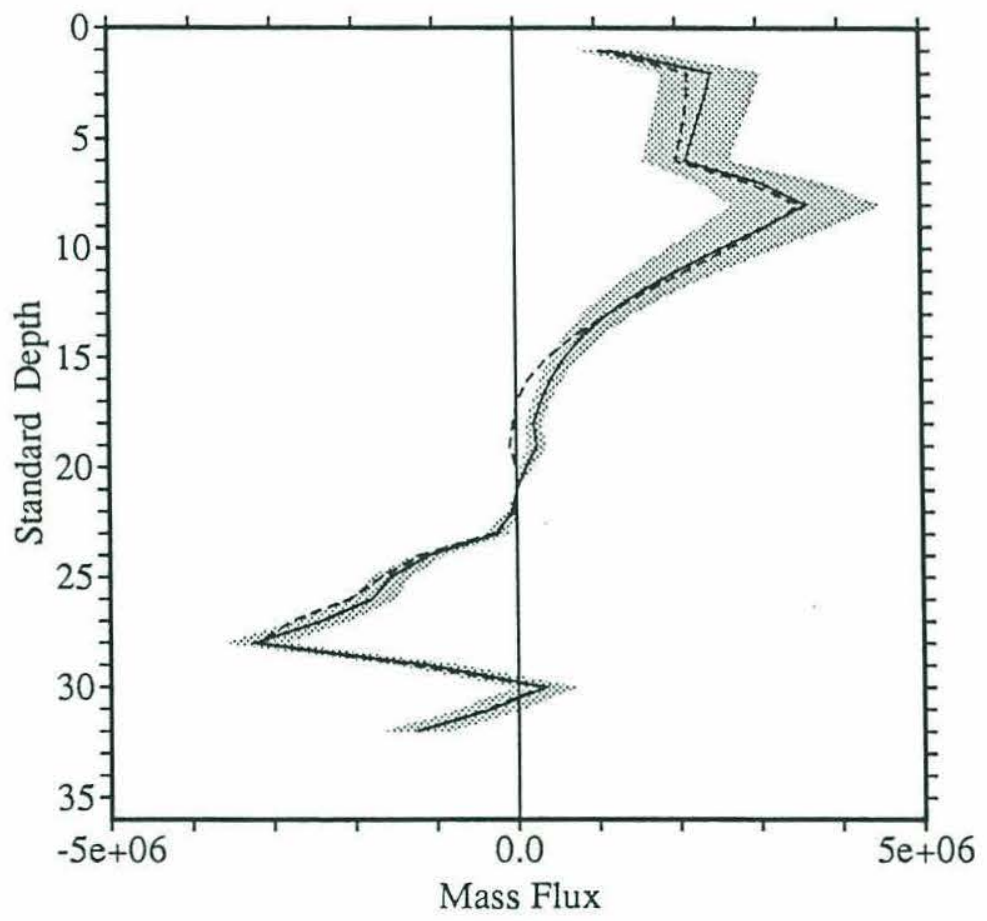

Figure 2-23. Mass Flux $\left(\mathrm{m}^{3} / \mathrm{s}\right)$ Profile of Atlantis II 109 leg 1 Different types of curves and shade are as in Figure 2-21.

(a.) mass flux between $8.9^{\circ} \mathrm{W}$ to $73.3^{\circ} \mathrm{W}$.

(b.) mass flux between $20.6^{\circ} \mathrm{W}$ to $73.3^{\circ} \mathrm{W}$. 
a. Dynamic Height

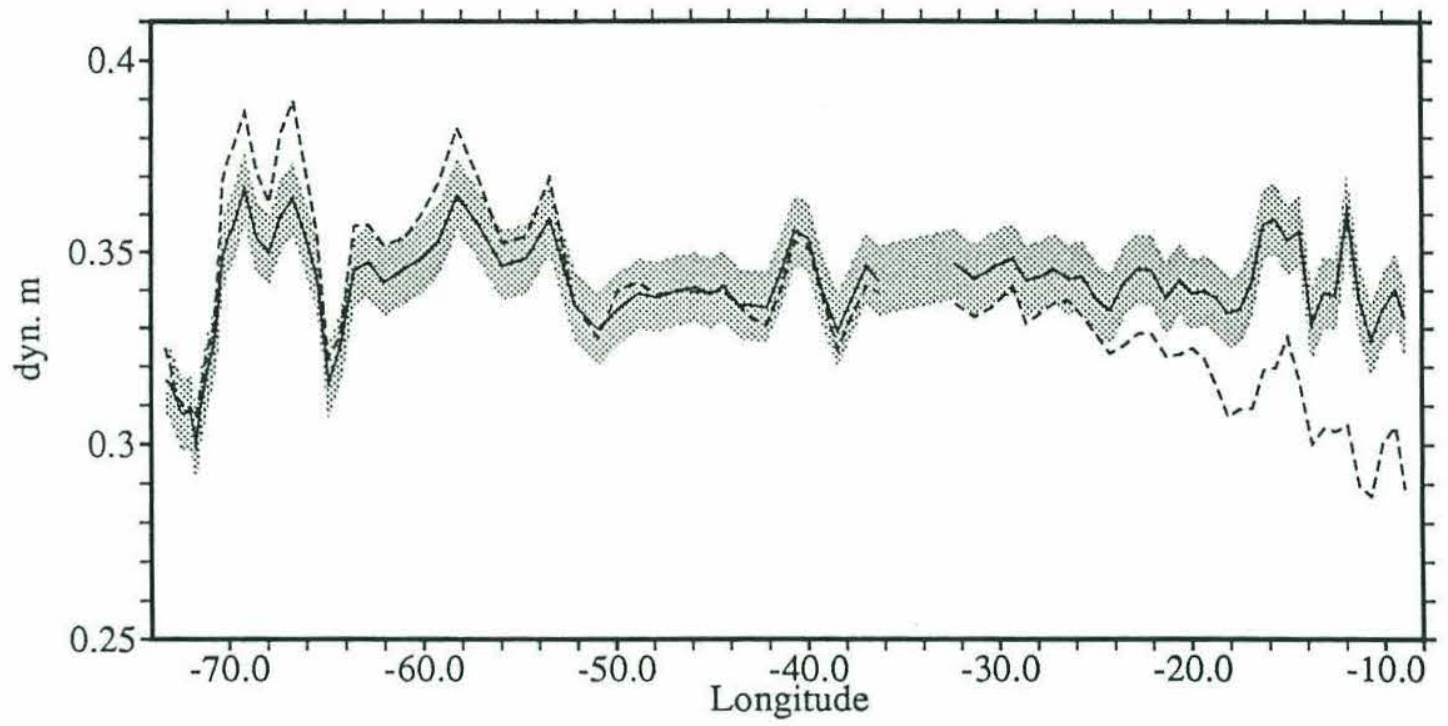

b. Mass Flux Difference between station pairs

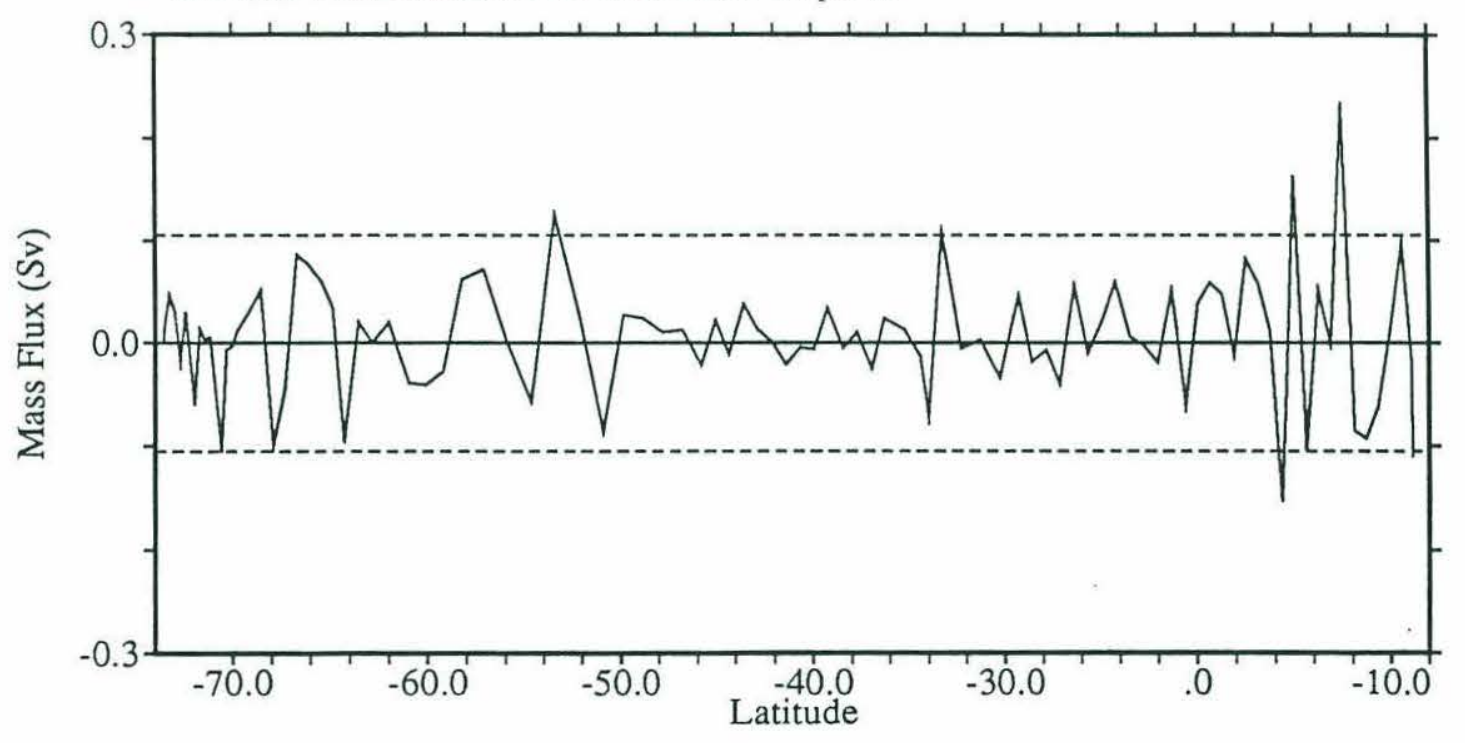

Figure 2-24. Comparison on Standard Depth 17 - Atlantis II 109 leg 1

(a.) Dynamic height referenced to depth 21 (2000 dbar). Different type of curves are as in Figure 2-21.

(b.) Mass flux difference between data and 6 mode reconstruction. Dashed line is the magnitude of error estimate. 


\section{Atlantis II 109 leg $3-24^{\circ} \mathrm{N}$}

The mass flux profile along this section between $75.4^{\circ} \mathrm{W}$ and $14.2^{\circ} \mathrm{W}$ is plotted in Figure 2-25. The mass flux of the modal reconstruction agrees with the data flux within the measure of error. An exception may be around 3000 dbar (25) where the difference is slightly larger than the error estimate. Figure 2-26 shows dynamic height at 3000 dbar along this section. The modal reconstruction's dynamic height along the section tends to diverge from the data towards the eastern half of the section (especially east of about $47^{\circ} \mathrm{W}$ ). However, the significance of the difference between modal reconstruction and data is marginal.

\section{Endeavor $129-64^{\circ} \mathrm{W}$}

Figure 2-27 shows the total mass flux profile along this section (between $18.9^{\circ} \mathrm{N}$ to $42.2^{\circ} \mathrm{N}$ ), where the error bars correspond to errors at $20^{\circ} \mathrm{N}$. There is a slight difference between modal model and data between $1100 \mathrm{dbar}(15)$ and $1500 \mathrm{dbar}$ (19), although marginal. Examination of dynamic height at 1300 dbar (17) along this section, Figure $2-28$, shows that this difference happens primarily at the southern end of this section, south of $21^{\circ} \mathrm{N}$. This is where the anomaly associated with the cold and fresh Antarctic Intermediate Water's influence becomes evident (the blob of fresh water at $1000 \mathrm{dbar}$ in Figure 1-2.)

\section{E. Oceanus $199 \operatorname{leg} 7-59^{\circ} \mathrm{W}$}

Figure 2-29a is total mass flux profile between $6.5^{\circ} \mathrm{N}$ and $43.2^{\circ} \mathrm{N}$, with error bars calculated for $10^{\circ} \mathrm{N}$. Most depths agree with the data except for, once again middepths between $1000 \mathrm{dbar}(14)$ and $1750 \mathrm{dbar}$ (20). Comparison of dynamic height on 1300 dbar (17), Figure 2-30, shows that this difference happens again towards the southern end, south of $15^{\circ} \mathrm{N}$. These latitudes are where the salinity anomaly associated with the Antarctic Intermediate Water becomes stronger and where Antarctic Bottom Water becomes evident at the bottom (Figure 2-2). A comparison between total mass 


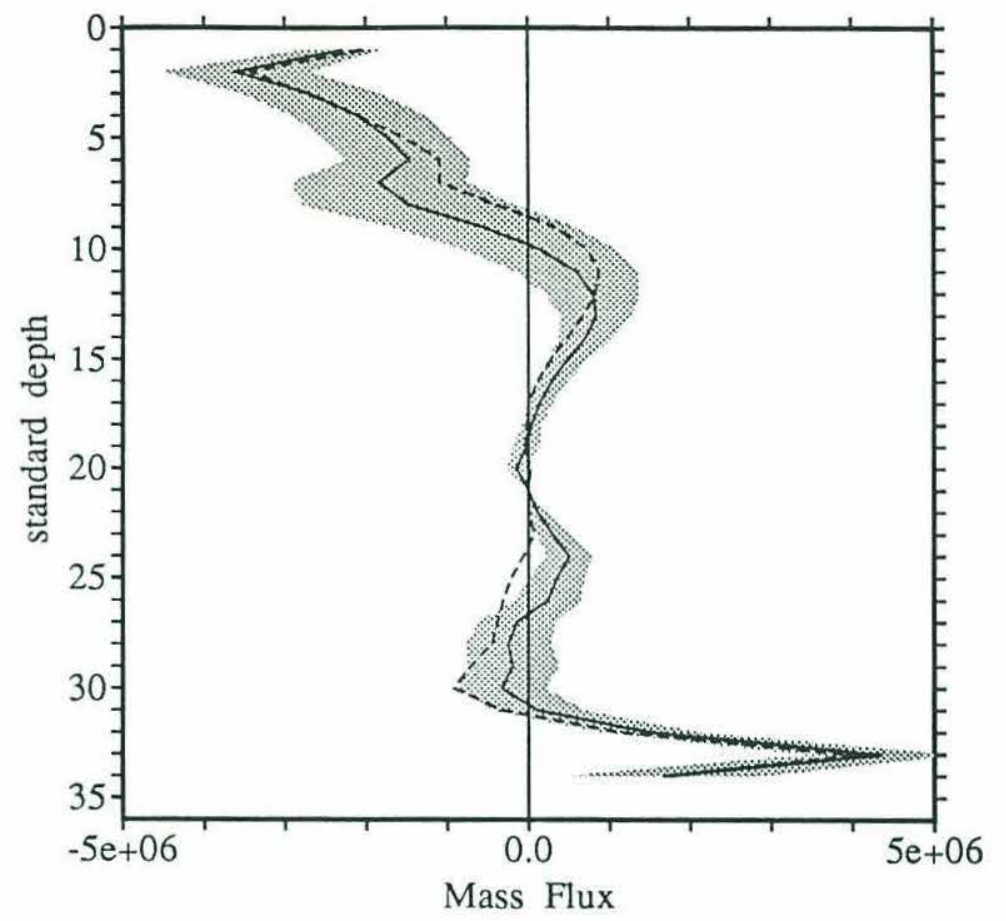

Figure 2-25. Mass Flux $\left(\mathrm{m}^{3} / \mathrm{s}\right)$ Profile of Atlantis II $109 \operatorname{leg} 3\left(75.4^{\circ} \mathrm{W}\right.$ to $\left.14.2^{\circ} \mathrm{W}\right)$

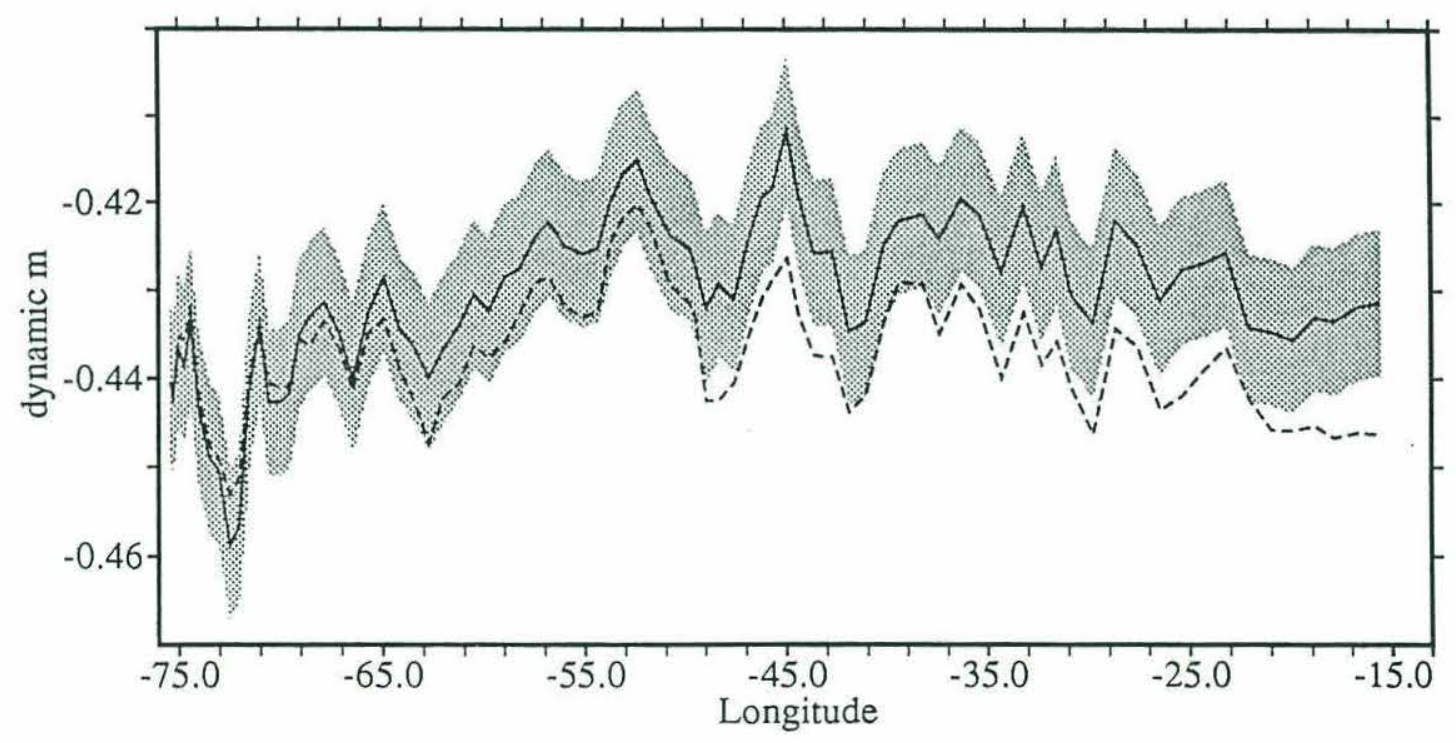

Figure 2-26. Dynamic Height Comparison on Depth 25 - Atlantis II 109 leg 3 


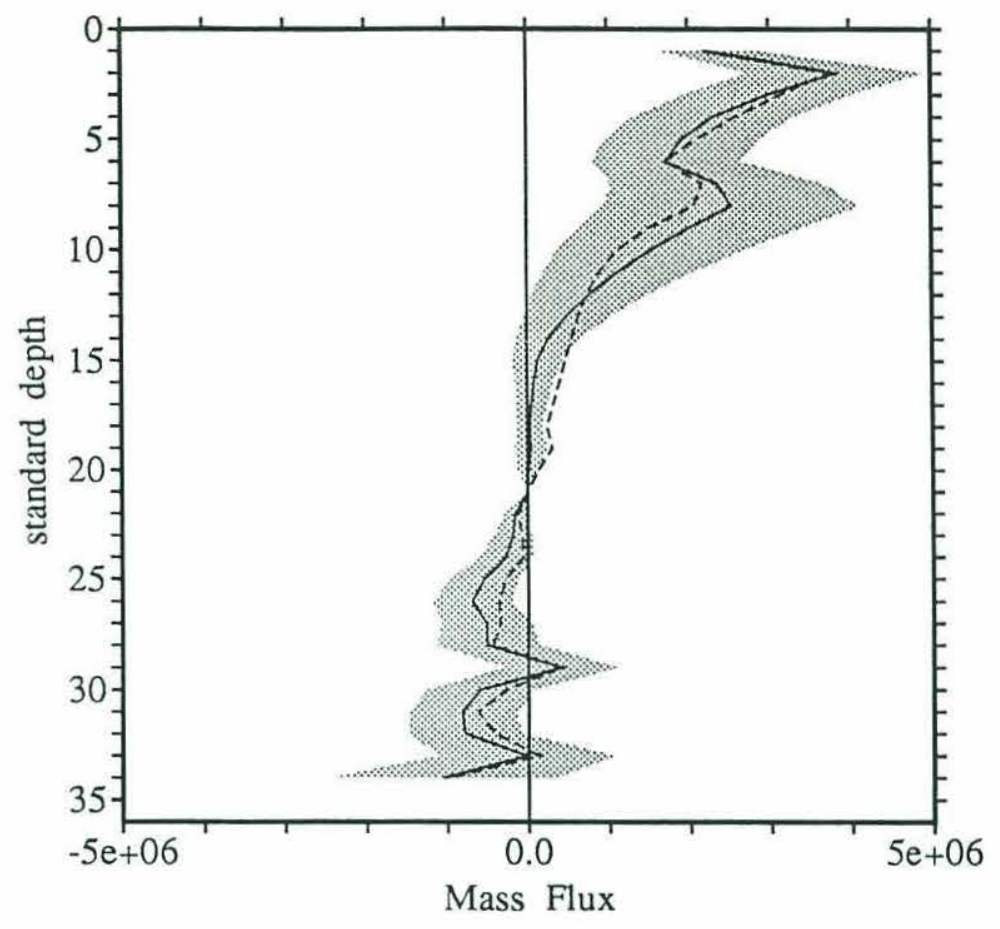

Figure 2-27. Mass Flux $\left(\mathrm{m}^{3} / \mathrm{s}\right)$ Profile of Endeavor $129\left(42.2^{\circ} \mathrm{N}\right.$ to $\left.18.9^{\circ} \mathrm{N}\right)$ Positive values correspond to eastward fluxes.

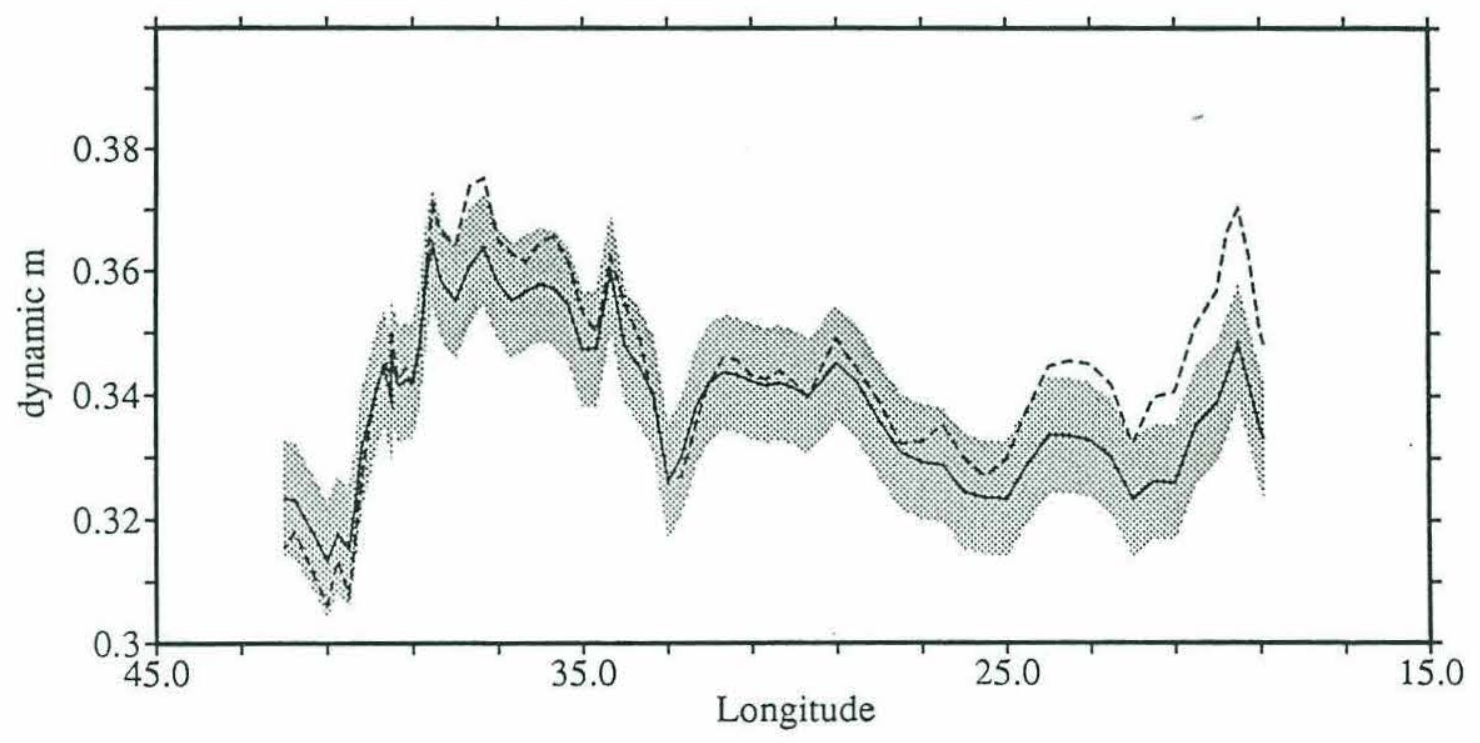

Figure 2-28. Dynamic Height Comparison on Depth 17 - Endeavor 129 
a. $43.2^{\circ} \mathrm{N}$ to $6.5^{\circ} \mathrm{N}$

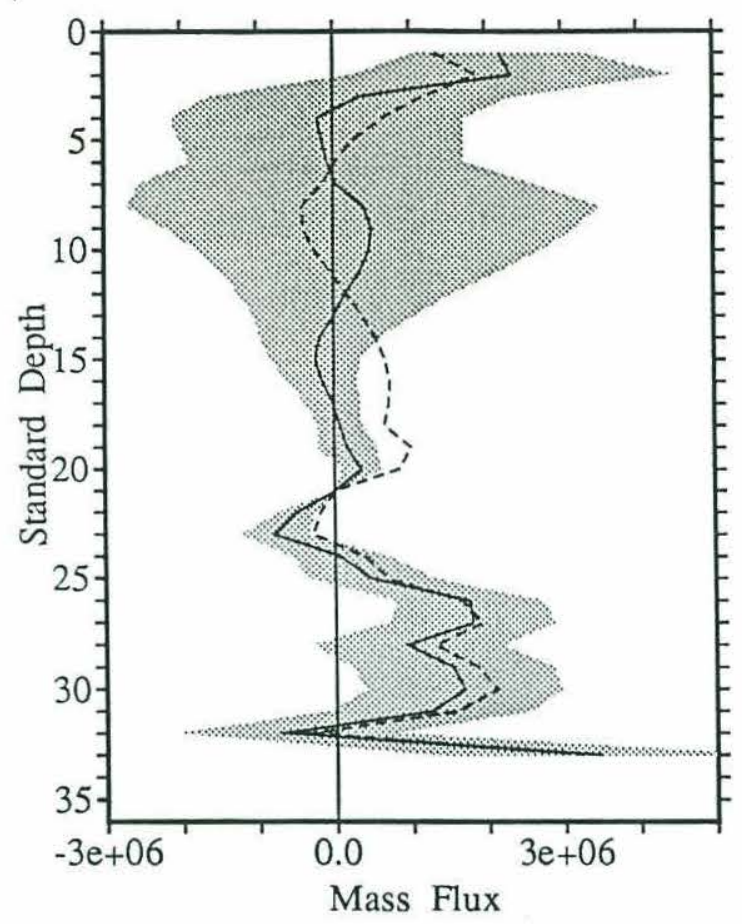

b. $43.2^{\circ} \mathrm{N}$ to $12.5^{\circ} \mathrm{N}$

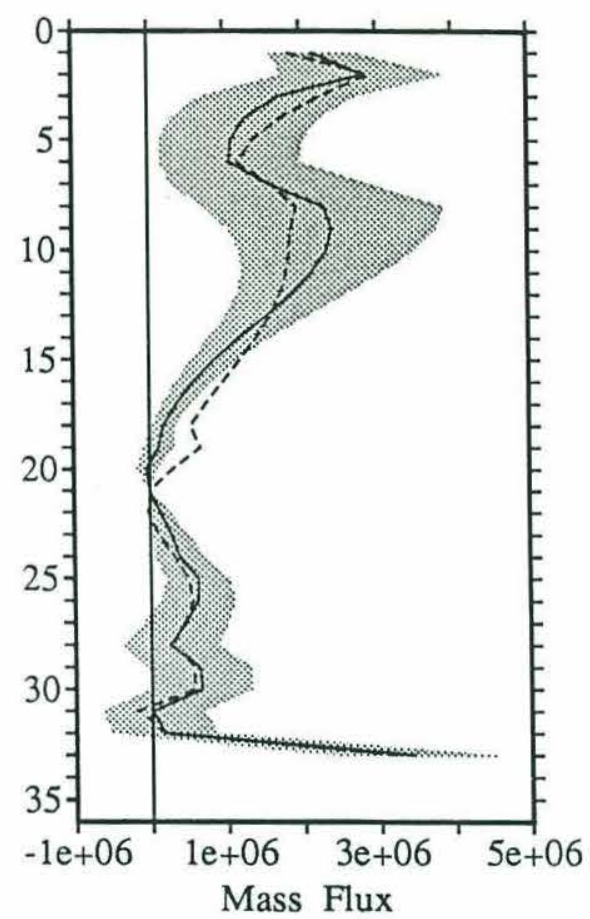

Figure 2-29. Mass Flux $\left(\mathrm{m}^{3} / \mathrm{s}\right)$ Profile of Oceanus $133 \mathrm{leg} 7$

(a) between $43.2^{\circ} \mathrm{N}$ to $6.5^{\circ} \mathrm{N}\left(\right.$ b) between $43.2^{\circ} \mathrm{N}$ to $12.5^{\circ} \mathrm{N}$

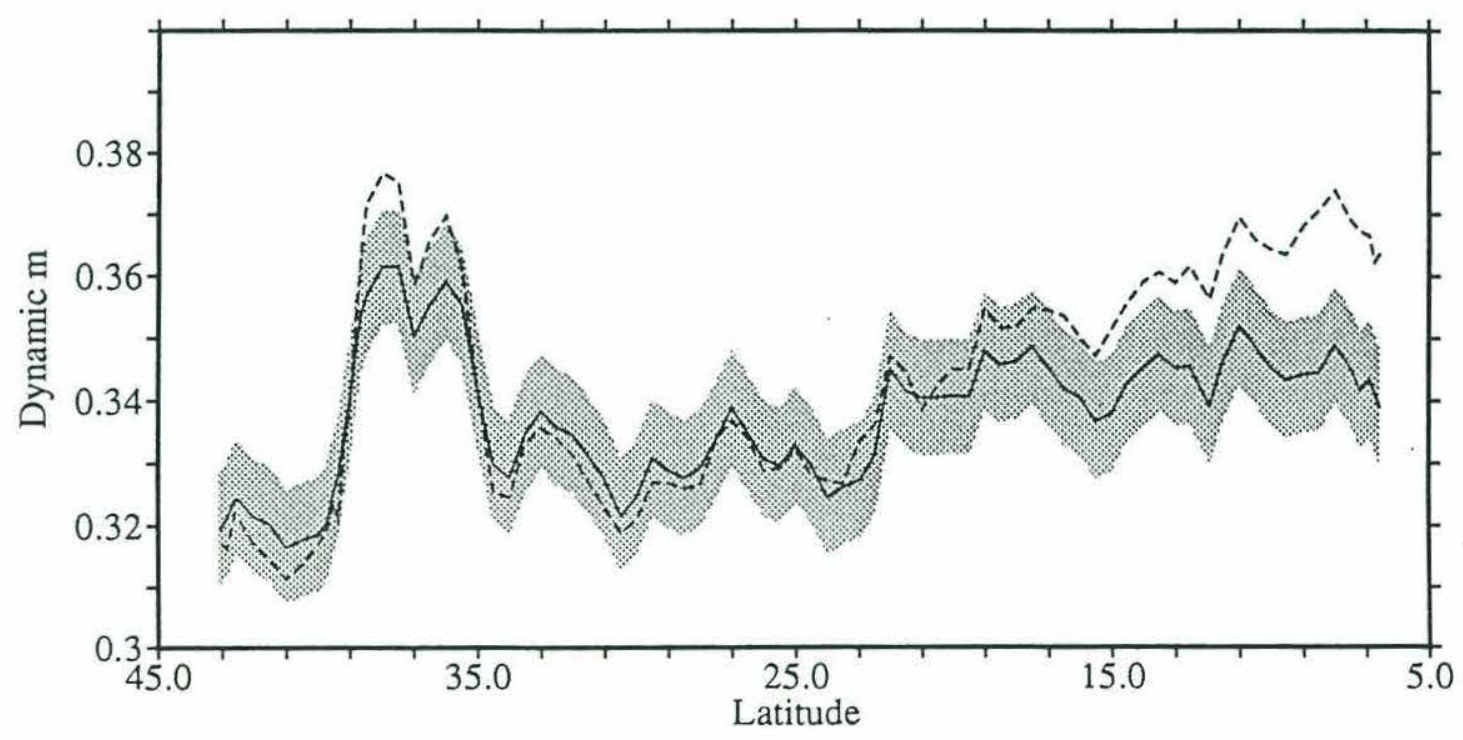

Figure 2-30. Dynamic Height Comparison on Depth 17 - Oceanus 133 leg 7 
fluxes between $12.5^{\circ} \mathrm{N}$ to $43.2^{\circ} \mathrm{N}$, Figure $2-29 \mathrm{~b}$, shows a smaller difference between modal reconstruction and data.

\section{F. Knorr $104-35^{\circ} \mathrm{W}$}

Total mass transport profile between $58.8^{\circ} \mathrm{N}$ and $5.5^{\circ} \mathrm{N}$ of this longest section is shown in Figure 2-31a (errors calculated at $10^{\circ} \mathrm{N}$ ). Any significant difference between data and modal model is again limited to mid-depths, between $1200 \mathrm{dbar}$ (16) to $1500 \mathrm{dbar}$ (19). Dynamic height comparison along 1500 dbar (19) (Figure 2-32) shows that the difference is not big throughout the section. However, there are regions where modal model dynamic height is slightly biased against the data; south of the North Atlantic Current $\left(48\right.$ to $40^{\circ} \mathrm{N}$ ), about $25^{\circ} \mathrm{N}$, and under the Antarctic Intermediate Water tongue (south of $11^{\circ} \mathrm{N}$ ). A comparison of total mass flux between $58.8^{\circ} \mathrm{N}$ and $12.6^{\circ} \mathrm{N}$ (Figure 2-31b) shows a better agreement between modal model and data.

\subsection{Discussion}

Any significant mass flux difference between modal model and data occur at middepths $(1000 \sim 2000 \mathrm{dbar})$ and in limited regions. The regions with largest bias are in the core of the Mediterranean outflow immediately west of the Strait of Gibraltar, and under the core of Antarctic Intermediate Water at tropical latitudes. Otherwise, the circulation of the modal model is in agreement with the original data to within a measure of noise.

The analysis shows that data and modal reconstruction agree well at shallower and deeper depths despite some of the biases at mid-depths. This result raises an interesting point. Mass flux is a function of dynamic height, which is a vertical integral of specific volume. The fact that mass flux is in agreement at shallow depths despite the differences below, indicates that the dynamic height bias at mid-depth becomes offset when integrated over large enough depth intervals. On the other hand, the modal fit is a best fit in a vertically integrated sense (equation 1-2a), and not to each separate 
a. $58.8^{\circ} \mathrm{N}$ to $5.5^{\circ} \mathrm{N}$

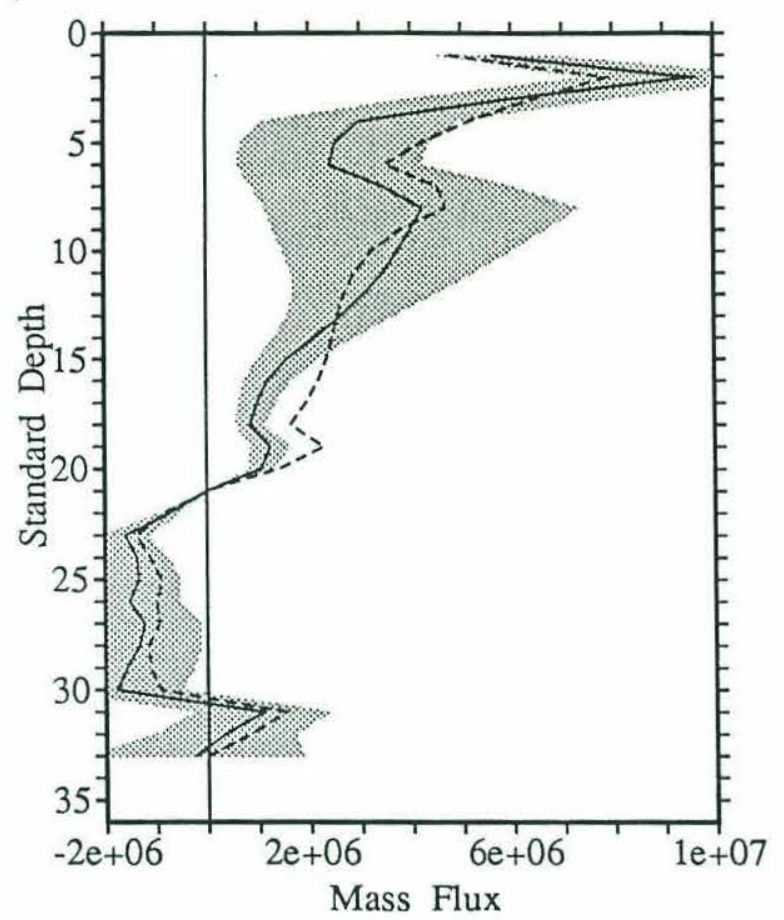

b. $58.8^{\circ} \mathrm{N}$ to $12.6^{\circ} \mathrm{N}$

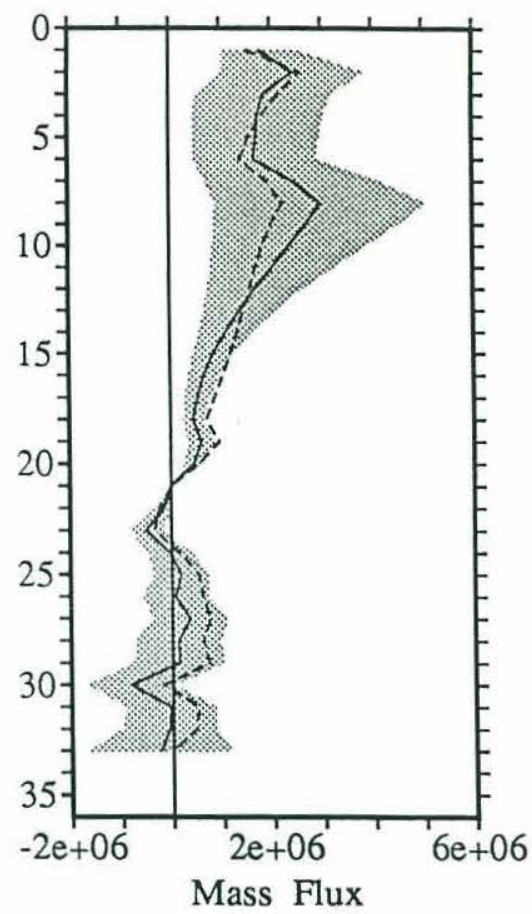

Figure 2-31. Mass Flux $\left(\mathrm{m}^{3} / \mathrm{s}\right)$ Profile of Knorr 104

(a) between $58.8^{\circ} \mathrm{N}$ to $5.5^{\circ} \mathrm{N}$ (b) between $58.8^{\circ} \mathrm{N}$ to $12.6^{\circ} \mathrm{N}$

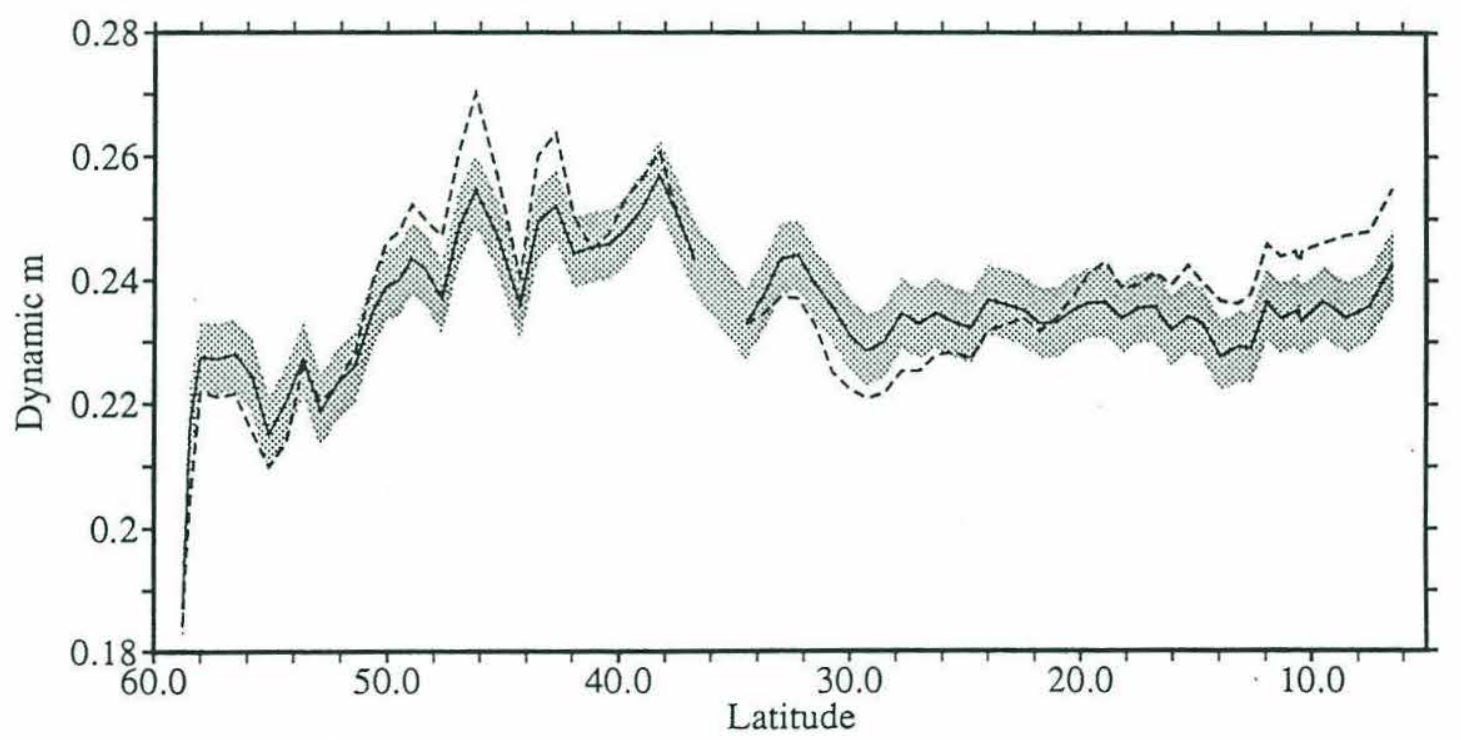

Figure 2-32. Dynamic Height Comparison on Depth 19 - Knorr 104 
depth. This is the reason for the apparent good fit of mass flux at shallow and deep depths.

In fact, it can be shown that much of the surface dynamic topography is accounted for by just the first two vertical modes. Figure 2-33 is a comparison of surface dynamic topography between the data and reconstructions based on the sum of modes 1 to 9 . Topography of the Gulf Stream and subtropical gyre are already reasonably reproduced after only the first two modes are included.

Figure 2-34 is the dynamic height perturbation of the first 10 modes referenced to 2000 dbar, as was defined by equations $1-19,1-20$, and $1-21$. (The expected values of the expansion coefficients were calculated as in equation 2-12 of Section 8.1) The first two modes have much larger surface perturbations than any of the higher modes and thus account for most of the sea-surface dynamic topography. (Higher modes are smaller because of the smaller singular values and the smaller vertical scales which effectively cancel when integrated vertically.)

This close agreement of surface topography contrasts with the lack of any such agreement of surface property as was seen in Section 5. For comparison surface temperature is reconstructed in Figure 2-35 for the same hydrographic sections as in Figure 2-33. Notice the increased scatter among different curves compared to those in Figure $2-33$.

These observations of reproduction of sea surface property by modes have an important implication with regard to remote sensing. The presence of large residuals in the low order modal reconstructions of the sea surface temperature implies that measurement of sea surface temperature alone will not allow an accurate prediction of subsurface hydrographic structure. On the other hand, observation of sea surface dynamic topography will greatly constrain the gravest two vertical modes. 
a. Knorr $104-35^{\circ} \mathrm{W}$

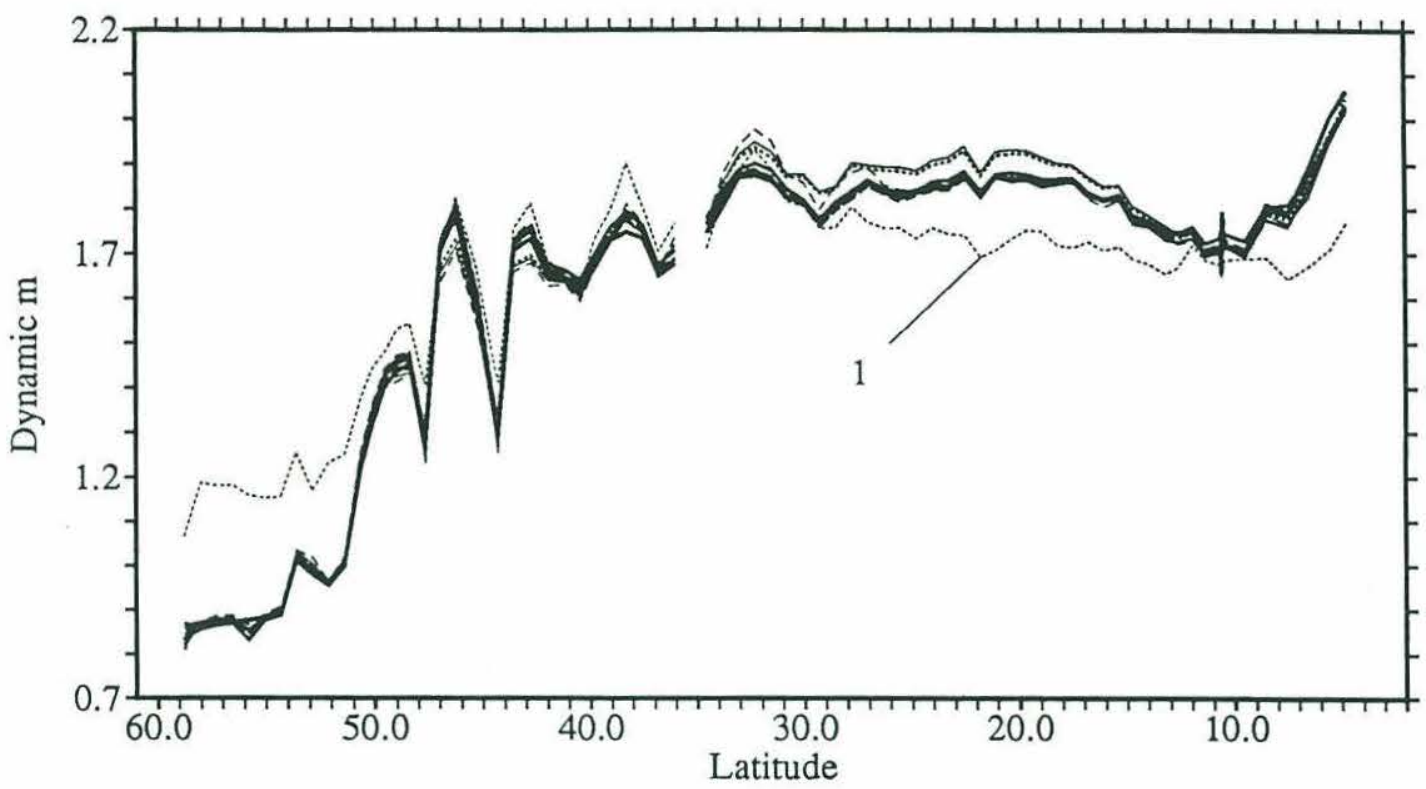

b. Atlantis-II $109-36^{\circ} \mathrm{N}$

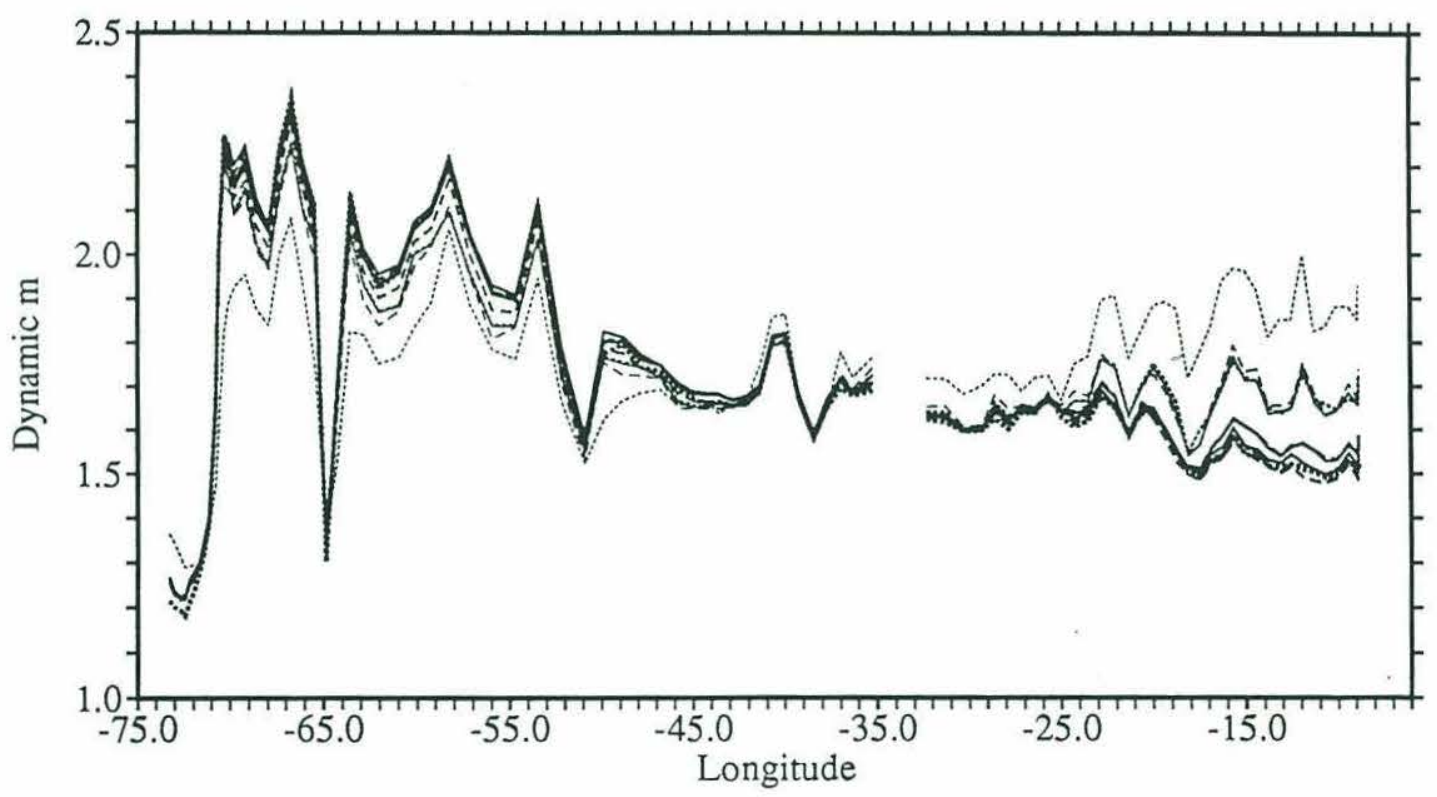

Figure 2-33. Sea Surface Topography Comparison

Topography are dynamic height referenced to 2000 dbar. Different curves correspond to different sum of modes. The curve labeled 1 is topography reproduced from only the first EOF. For legends on other curves refer to Figure 2-35 where curves are more legible.
(a) Knorr $104\left(35^{\circ} \mathrm{W}\right)$
(b) Atlantis II 109 leg $1\left(36^{\circ} \mathrm{N}\right)$ 


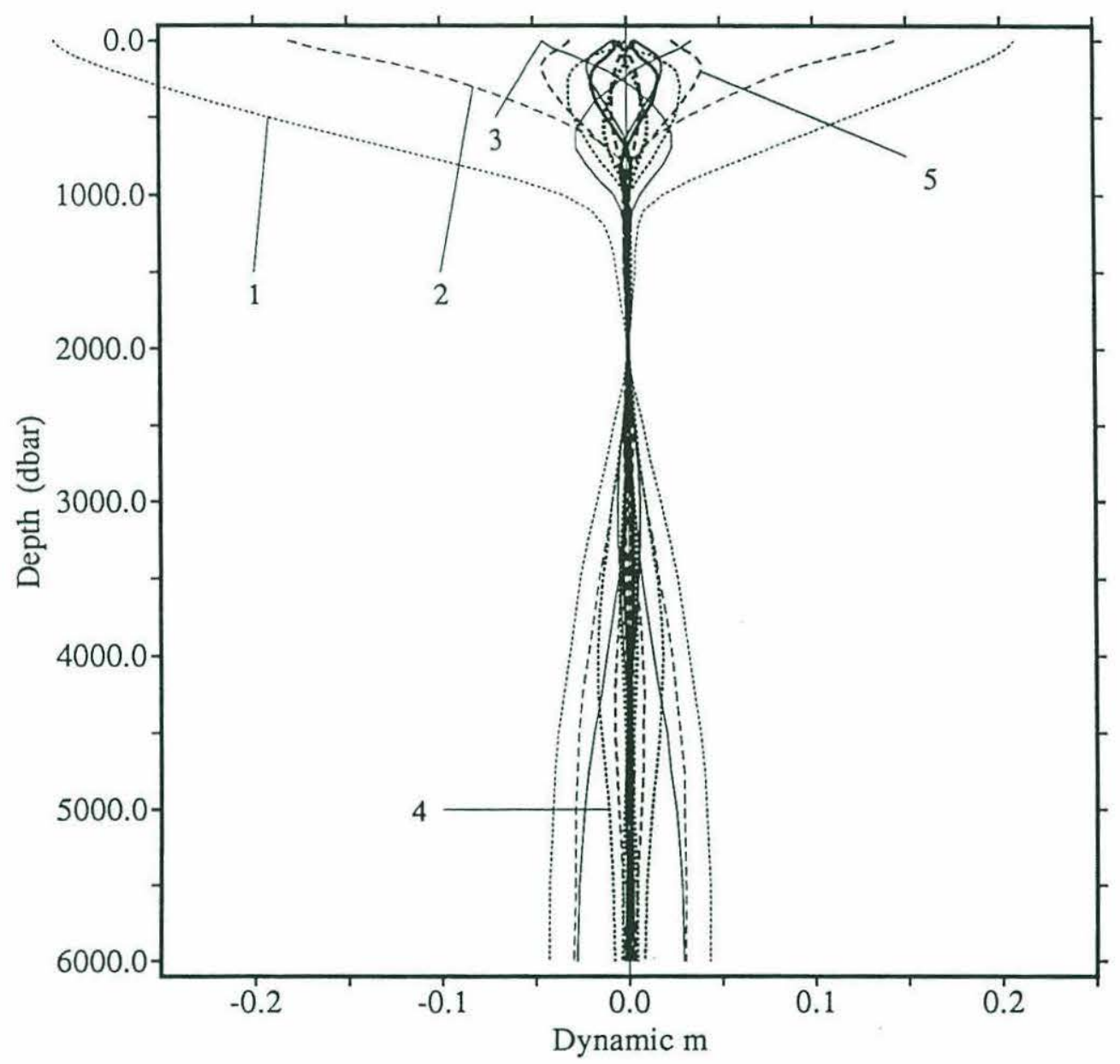

Figure 2-34. Modal Dynamic Height Perturbations

The profiles show the perturbations of each EOF to the mean dynamic height, using average expansion coefficient. 
a. Knorr $104-35^{\circ} \mathrm{W}$

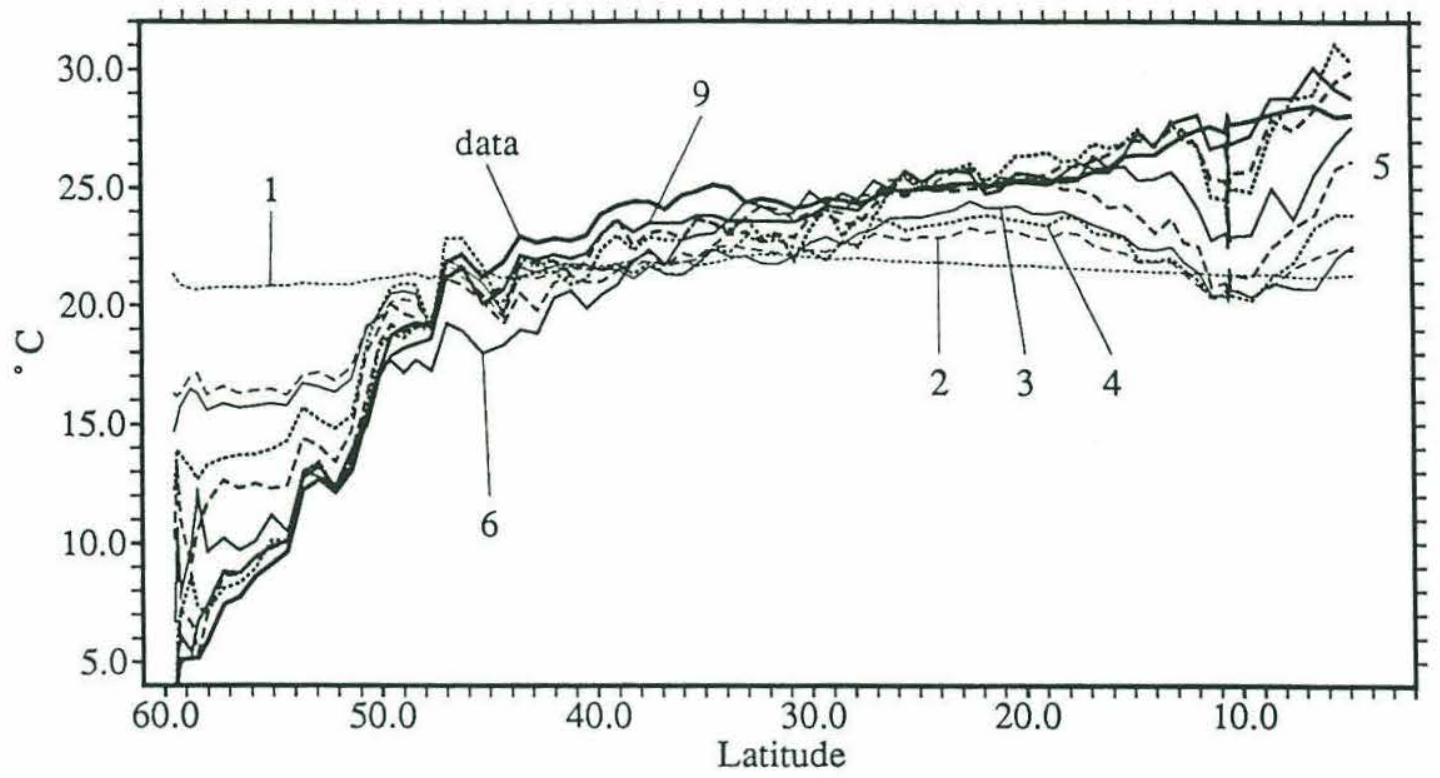

b. Atlantis-II $109-36^{\circ} \mathrm{N}$

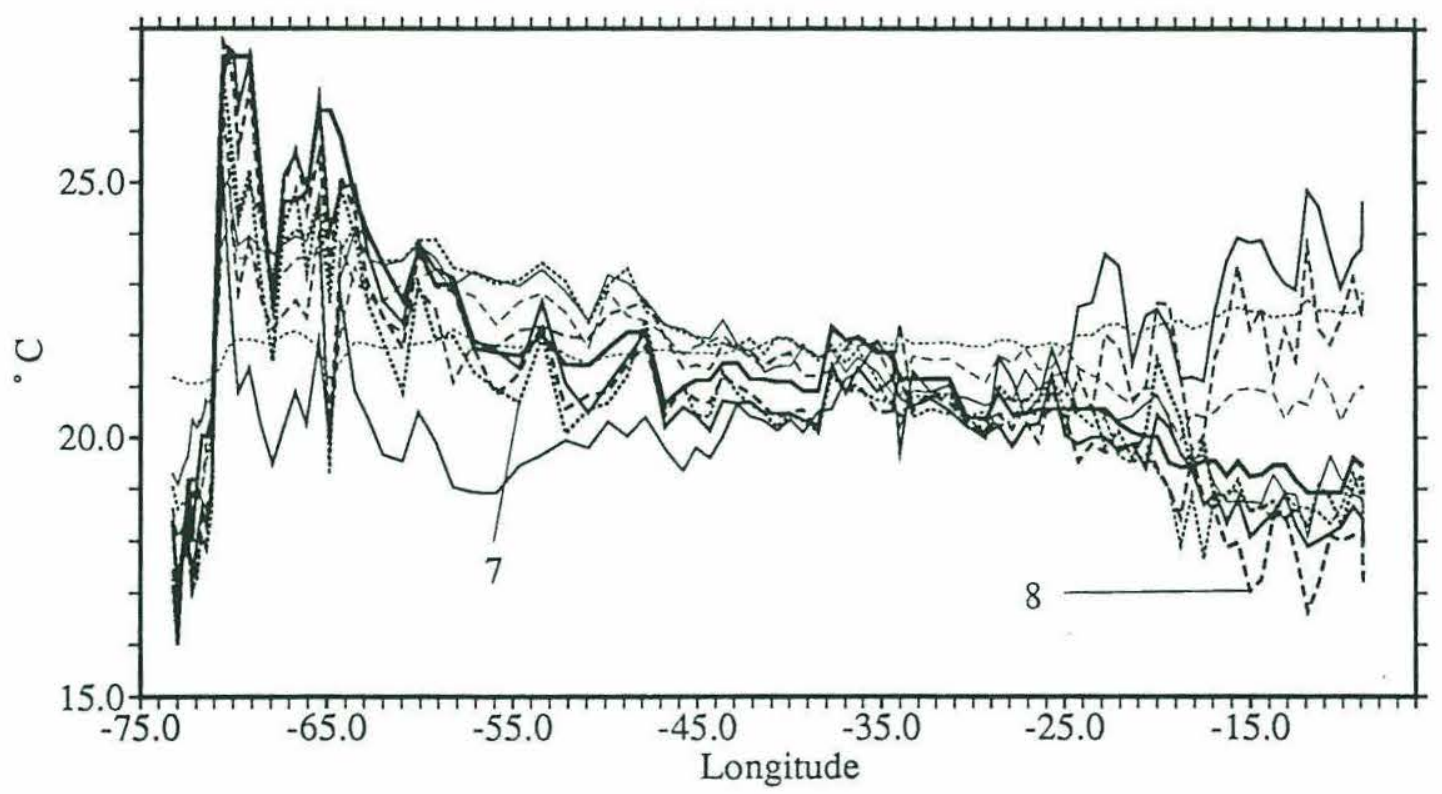

Figure 2-35. Sea Surface Temperature Comparison

Sea surface temperature is compared between the original data and reconstruction by various sum of modes. Each type of curve is labeled as what the maximum number of modes are.
(a) Knorr $104\left(35^{\circ} \mathrm{W}\right)$
(b) Atlantis II $109 \operatorname{leg} 1\left(36^{\circ} \mathrm{N}\right)$ 


\section{Modal Coefficients}

\subsection{Mapping onto a Regular Grid}

In the previous sections, we have seen that the first 6 modes account reasonably well for the dominant hydrographic structure and circulation of the North Atlantic Ocean when compared to our measure of error. Thus, synthesis of the large volume hydrographic data can be done efficiently based on the expansion coefficients of these 6 modes. An example of data synthesis is to map the hydrographic structure of the entire ocean on a regular grid from irregularly spaced station data. Rather than having to map on each of the 35 standard depths, we can readily do the mapping from the 6 modal coefficients and still resolve the major features of the ocean structure.

Figure 2-36 shows mode 1 expansion coefficient of temperature. Despite some short scale horizontal variations, the large scale coherence is apparent. This expansion coefficient is positive north of the Gulf Stream-North Atlantic Current and in the tropical latitudes south of $10^{\circ} \mathrm{N}$. It is negative at mid latitudes with larger magnitudes towards the eastern end.

Objective mapping (Bretherton et al., 1976) is used to map the expansion coefficients on every degree latitude and longitude over the North Atlantic Ocean. The data covariance was modeled by a Gaussian covariance function with an $e$-folding scale of $1000 \mathrm{~km}$. Data variance is modeled from the expected square value of the expansion coefficient and a model of noise.

From the way we normalize the data to be nondimensional, the expected square values of the expansion coefficients are uniform among different modes and different variables. However, since the columns are weighted in calculating the modes, so that the number of stations per unit area is uniform among the variables and basins, the expansion coefficients do not form an orthonormal set as they did in Chapter 1. Normalization is done relative to temperature in basin 1 , where this rate is $3.10 \times 10^{4}$ $\left(\mathrm{km}^{2} /\right.$ station). Total area of the 6 basins is $3.31 \times 10^{7}\left(\mathrm{~km}^{2}\right)$. So the effective number 


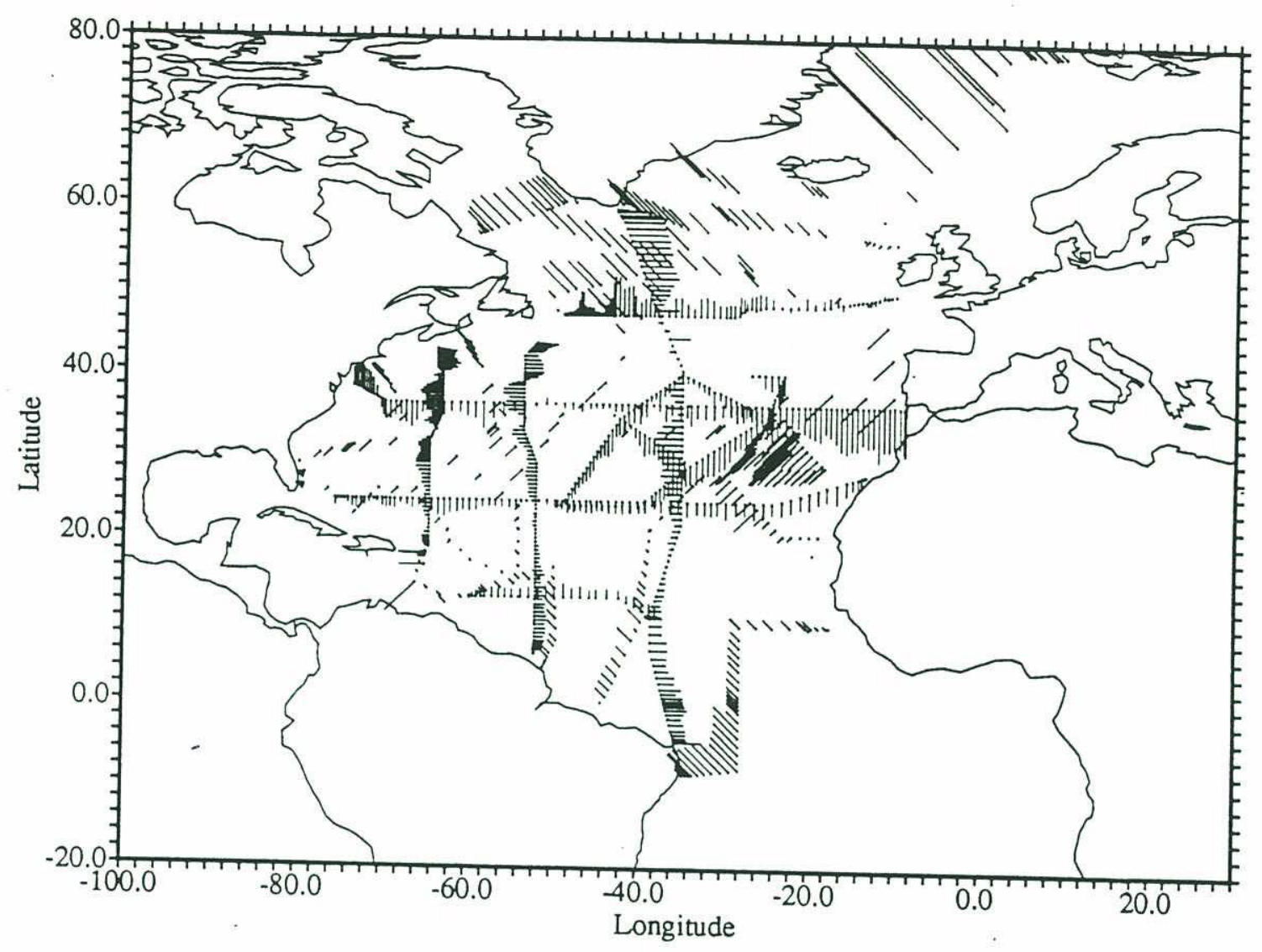

Figure 2-36. Mode 1 Temperature Expansion Coefficient Sticks with northward (southward) components are positive (negative).

\begin{tabular}{|c|c|c|c|}
\hline mode & $s_{i}$ & $n_{e}$ & $n_{l}$ \\
\hline 1 & 1.18 & 0.22 & 0.07 \\
\hline 2 & 1.13 & 0.37 & 0.13 \\
\hline 3 & 1.11 & 0.43 & 0.15 \\
\hline 4 & 1.12 & 0.40 & 0.19 \\
\hline 5 & 1.08 & 0.44 & 0.27 \\
\hline 6 & 1.01 & 0.55 & 0.33 \\
\hline
\end{tabular}

Table 2-5. Modal Expansion Coefficient Statistics The values are square root variances $\left(\times 10^{-2}\right)$ of the modal expansion coefficients' signal $\left(s_{i}\right)$, and noise $\left(n_{e}, n_{l}\right)$. 
of stations is 1068.8 , making the effective number of elements of the entire expansion coefficients to be $1068.8 \times 6=6412.8$. The expected rms value of each expansion coefficient is thus,

$$
\sqrt{\left\langle v_{i}^{2}\right\rangle}=\frac{1}{\sqrt{6412.85}}=1.4 \times 10^{-4} .
$$

Each expansion coefficient is the sum of the signal, $s_{i}$, and noise. Noise will be modeled by two parts, $n_{l}$ and $n_{e}$. Assuming each component to be independent of one another, $<v_{i}^{2}>$ can be written,

$$
<v_{i}^{2}>=<s_{i}^{2}>+<n_{l}^{2}>+<n_{e}^{2}>
$$

The first part of noise, $n_{l}$, is the variance caused by what we shall collectively call local measurement error, which will be modeled by a covariance function with zero correlation distance. Variations that are spatially local, or variations caused by temporal aliasing will be included. An expansion coefficient at a particular station is,

$$
v_{i}=\frac{1}{\lambda} \underline{u}^{T} \underline{t}_{i}
$$

We have seen that with the first 6 modes, the RMS error is on the order of 0.5 at most depths. Assuming this to be a reasonable measure of measurement error for $\underline{t}_{i}$ and that this error at one depth is independent of another depth, the local error $n_{l}$ can be estimated by,

$$
n_{l}=\frac{0.5}{\lambda}
$$

since $\underline{u}$ is orthonormal.

The second part of noise, $n_{e}$, is the variation caused by mesoscale eddies. $n_{e}$ has a finite correlation distance as opposed to the local error, $n_{l}$, but this distance is much smaller than the correlation distance of the large scale signal we are trying to map. Statistics of this mesoscale disturbance is modeled again by a Gaussian covariance function which has a correlation distance of $200 \mathrm{~km}$. The variance due to mesoscale 
variability is estimated in a similar manner to the measure of variable normalization (Section 3). The total noise (sum of $n_{l}$ and $n_{e}$ ) was estimated by equation 1-9 by the average over station separation of 1 to 5 . This gives a value nearly equal to 3 station separations which is about $175 \mathrm{~km}$. The variance of signal $\left(s_{i}^{2}\right)$, measurement noise $\left(n_{l}^{2}\right)$, and eddy noise $\left(n_{e}^{2}\right)$ are summarized in Table 2-5. The difference of $n_{e}^{2}$ 's among the 6 variables are found to be relatively small and so the mean values among the six variables are used. These variances are assumed independent of different variables and dependent only on different modes.

Objective mapping of the first 6 modal expansion coefficients were performed using all stations except those in the Caribbean Sea and the Norwegian Sea, because of the large difference in the deep water characteristics below the sill depth which separate these seas with the open North Atlantic. Estimates are made every degree in latitude and in longitude from $60^{\circ} \mathrm{N}$ to the southern boundary of basin 3 and 4 (Figure 2-7).

Estimates are also made at original data locations to examine if the chosen statistics are reasonable. Figure 2-37 compares some of the original expansion coefficients with the objectively mapped coefficients along some sections. For Knorr 104, both the gravest and the sixth mode coefficients are interpolated reasonably and the difference between the mapped result and data is overall consistent with our a priori errors. On the other hand the mapped result across the Gulf Stream for Endeavor 129 is not as sharp as the original data. This is due to the long correlation distance of our signal covariance function. To properly resolve the Gulf Stream at such longitudes which has a cross-stream width of $100 \mathrm{~km}$ or so, a smaller cross-stream correlation distance would have been more appropriate. However, the rest of the section is mapped reasonably smoothing over the mesoscale variations; and the gross structure of the Gulf Stream is also resolved. We conclude that the statistics employed are reasonable overall, not grossly unrealistic, but with the reservation that the mapping is over-smoothing the Gulf Stream. 
a. Knorr $104-35^{\circ} \mathrm{W}$ T mode 1

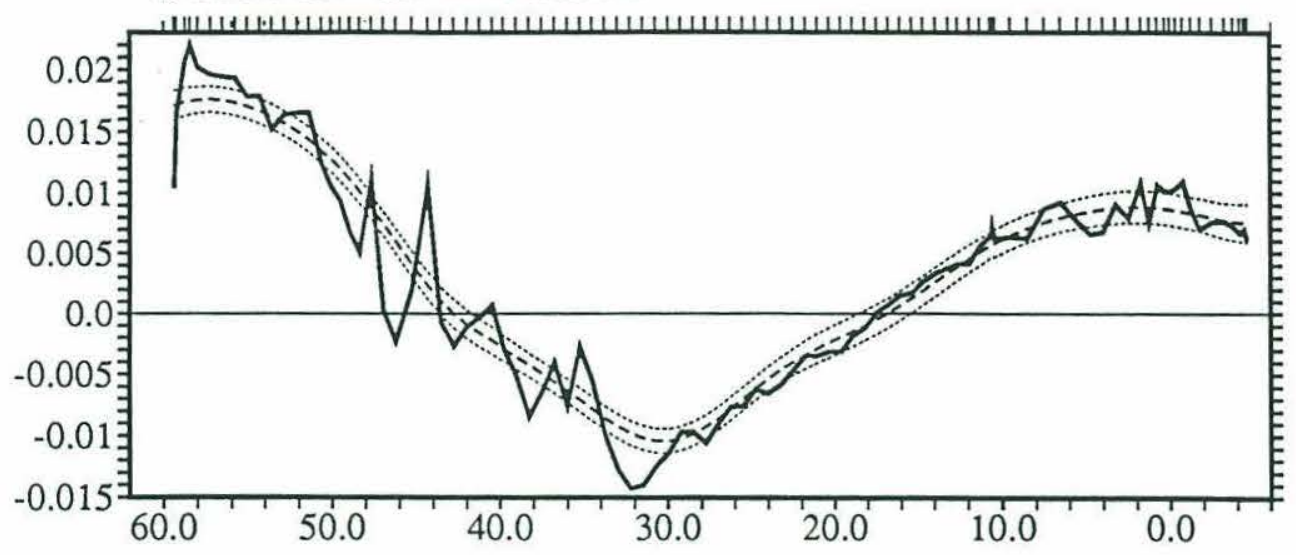

b. Knorr $104-35^{\circ} \mathrm{W}$ T mode 6

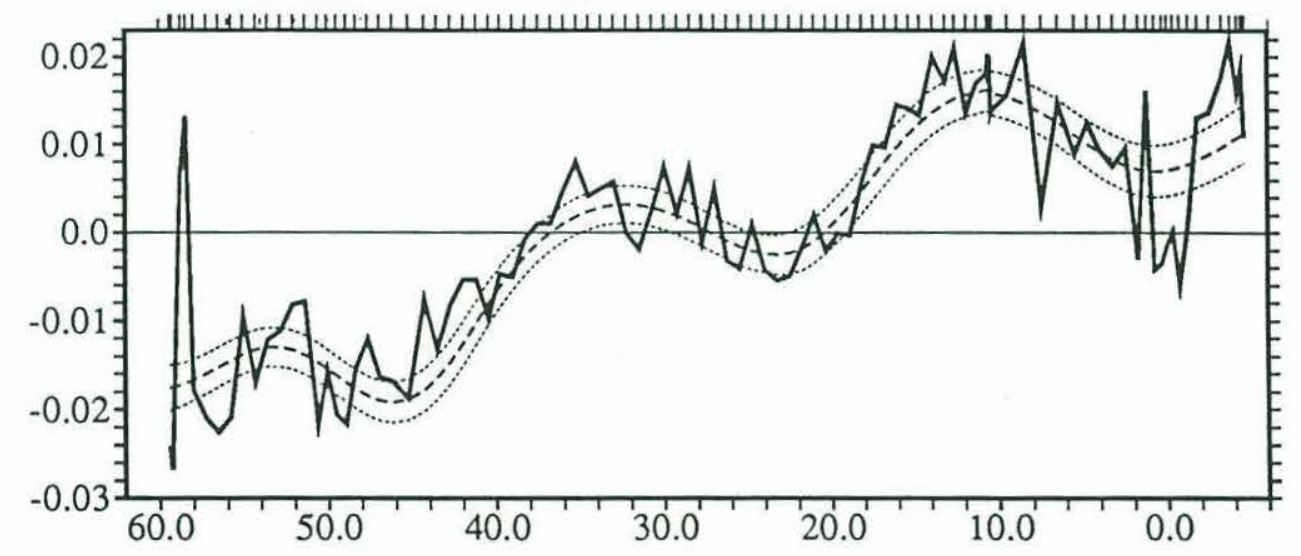

c. Endeavor $129-64^{\circ} \mathrm{W} T$ mode 1

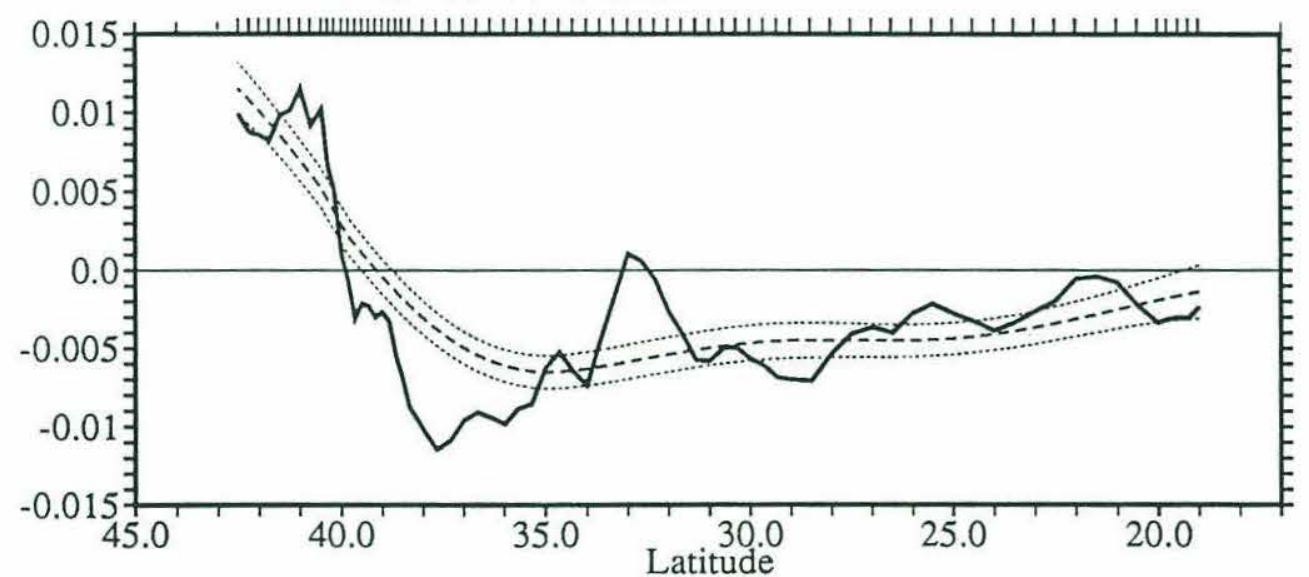

Figure 2-37. Comparison of Mapped Expansion Coefficient The curves are the original expansion coefficients of the data (solid), the mapped coefficients (dashed), and mapped value plus/minus the mapped error estimate (dotted). (a) Temperature mode 1 along Knorr 104 (b) Temperature mode 6 along Knorr 104 (c) Temperature mode 1 along Endeavor 129 


\subsection{Mapped Expansion Coefficients}

Figure 2-38 shows the first 3 modal coefficients of temperature and silicate. Note that the general character of temperature coefficient does have the coherent pattern we see in the raw expansion coefficient, Figure 2-36. The general pattern of these coefficients are reminiscent of the general hydrographic structure of the North Atlantic Ocean.

The negative "tongue" like structure emanating from Spain in mode 1 temperature reminds us of the Mediterranean Water spreading from the Strait of Gibraltar at some depth. The structure of the first vertical mode is negative throughout the depth (Figure 2-9). In essence, this first mode accounts for the vertical mean variation of property. Thus, a negative first mode expansion coefficient implies that water is warmer in this tongue than the rest of the ocean, consistent with warmer and saltier waters of Mediterranean origin. The positive coefficients north of the negative patch would correspond to the colder waters north of the Gulf Stream.

The pattern is quite different for the first mode silicate coefficients. The vertical mean just has a north-south gradient, suggestive of the contrast between nutrient poor North Atlantic origin waters to the north (positive coefficient) and nutrient rich Antarctic origin waters to the south (negative coefficient). It is interesting to note that the ridge of the high silicate coefficient (Figure 2-38b) is following the western boundary southward, which is indicative of the North Atlantic Deep Water spreading south as a boundary current.

The second vertical mode has a zero crossing at about $1000 \mathrm{dbar}$ (14) with negative values at shallow depths. So, the negative patch in temperature (Figure 2-38c) at mid latitudes suggests that water is warmer above $1000 \mathrm{dbar}$ and colder below corresponding to a stronger and deeper thermocline at these locations, indicative of the "bowl-shaped" thermocline of the subtropical gyre. Second mode silicate has a high at subtropical latitudes, which makes the shallow levels lower in silicate. Indeed the shallow levels do have the lowest nutrient concentration at subtropical latitudes (Section 2). 
(a)

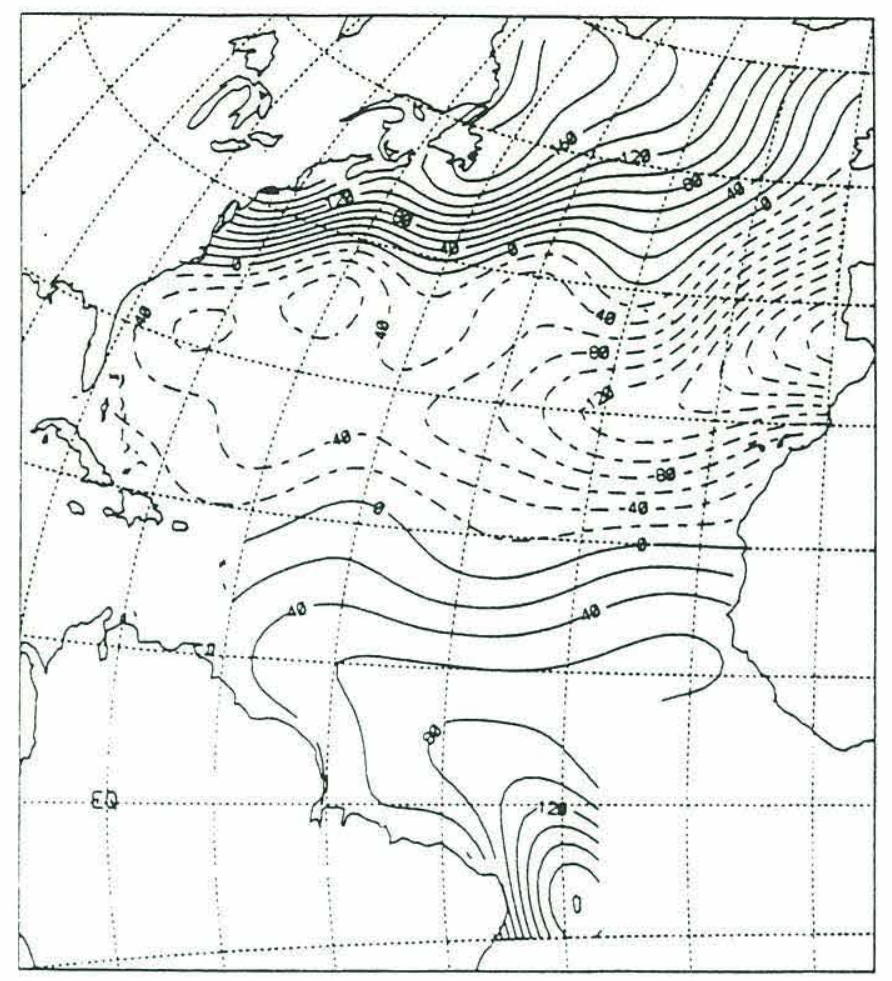

(b)

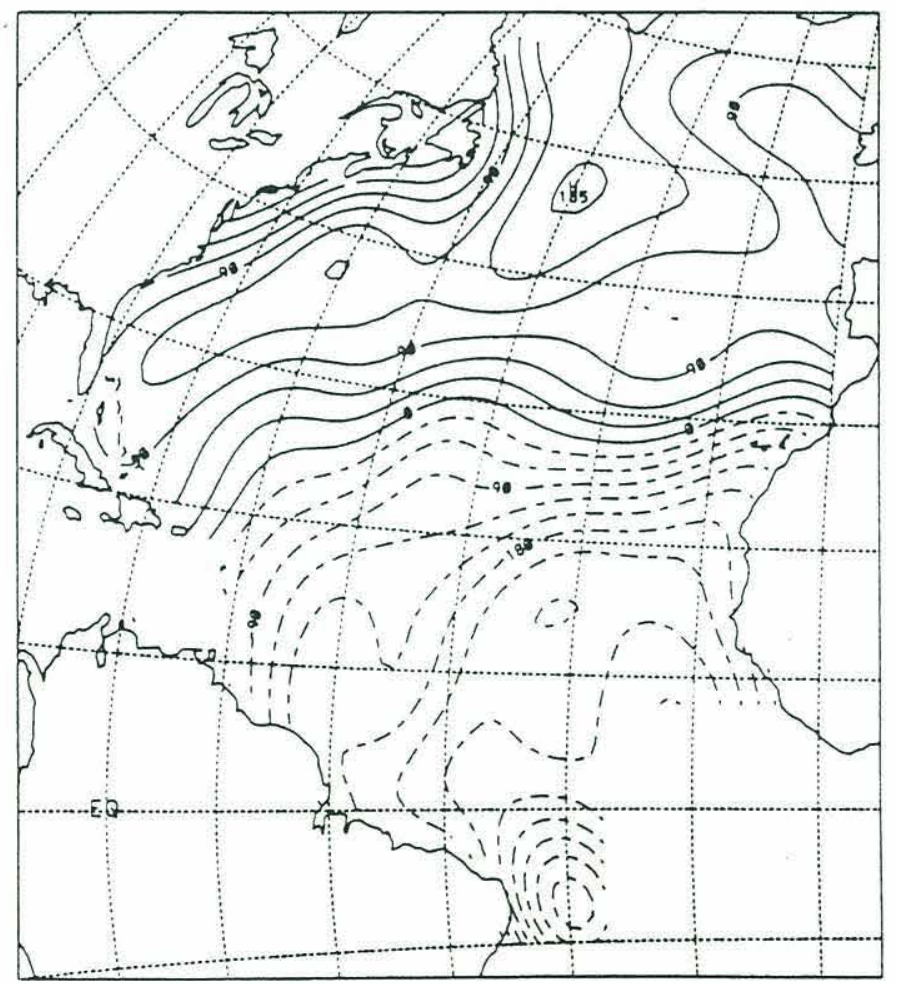

Figure 2-38. Mapped Expansion Coefficient $\left(\times 10^{-4}\right)$

(a) Temperature Mode 1 (b) Silicate Mode 1 
(c)

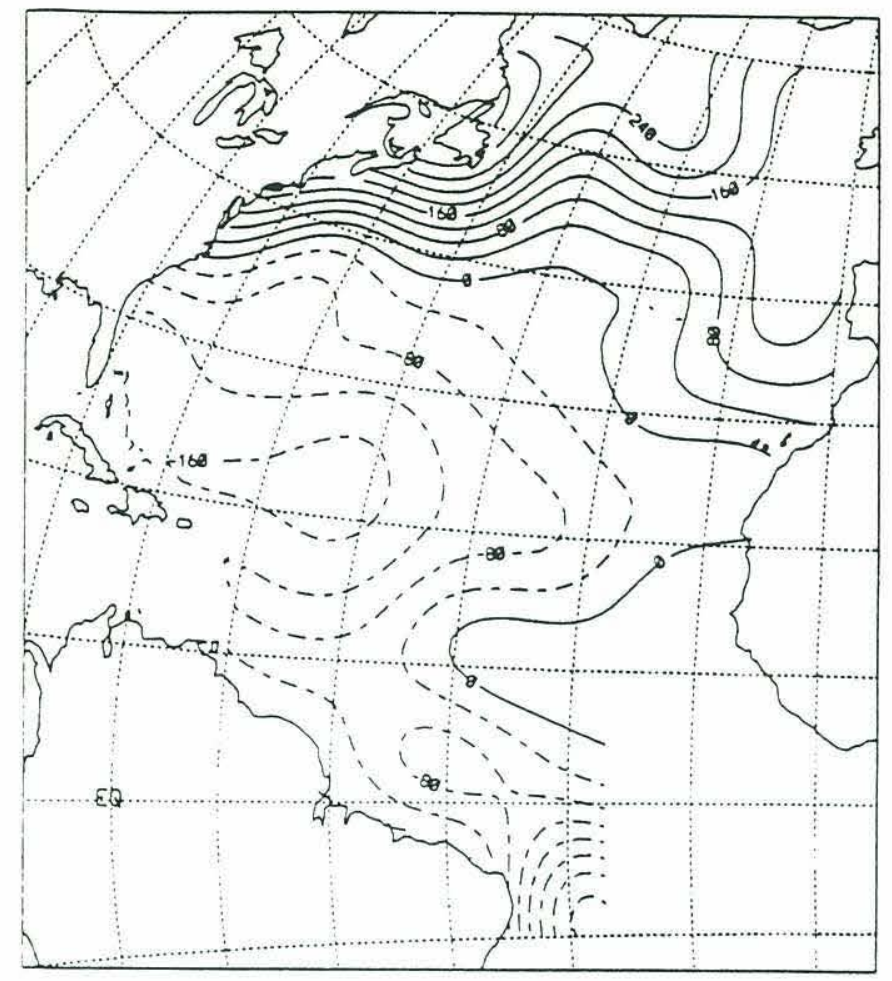

(d)

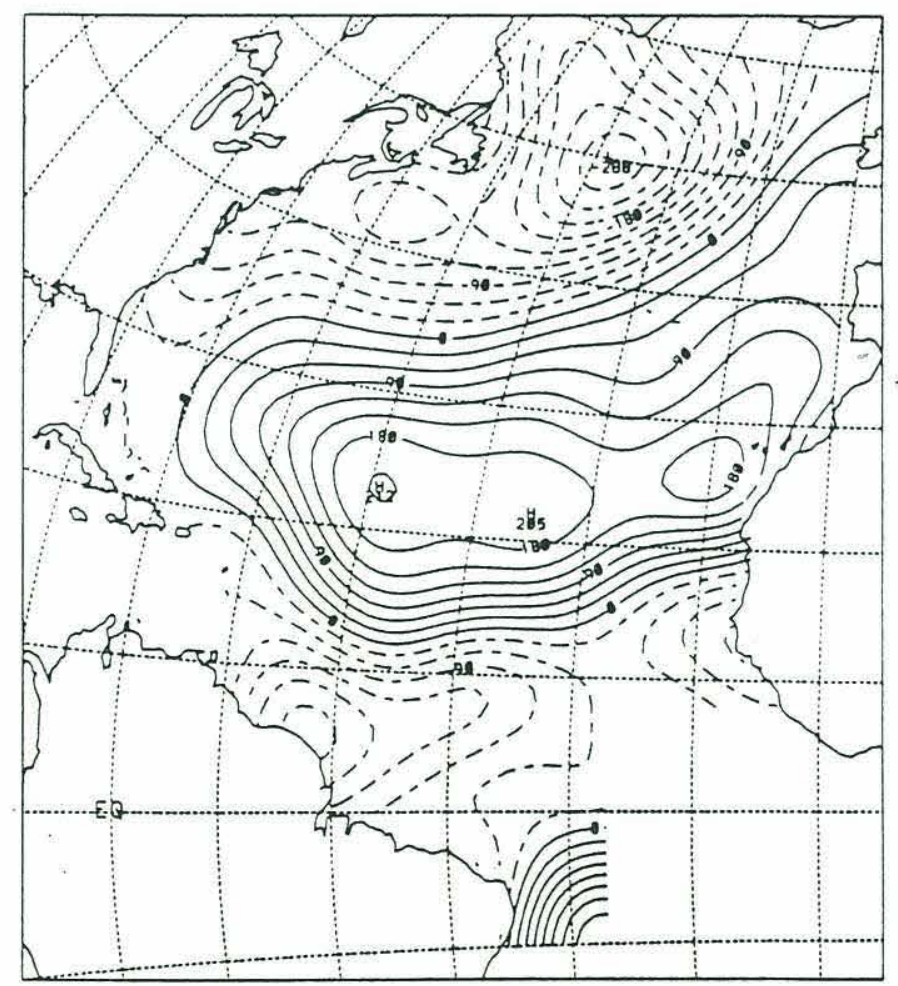

Figure 2-38. (continued) Mapped Expansion Coefficient $\left(\times 10^{-4}\right)$ (c) Temperature Mode 2 (d) Silicate Mode 2 
(e)

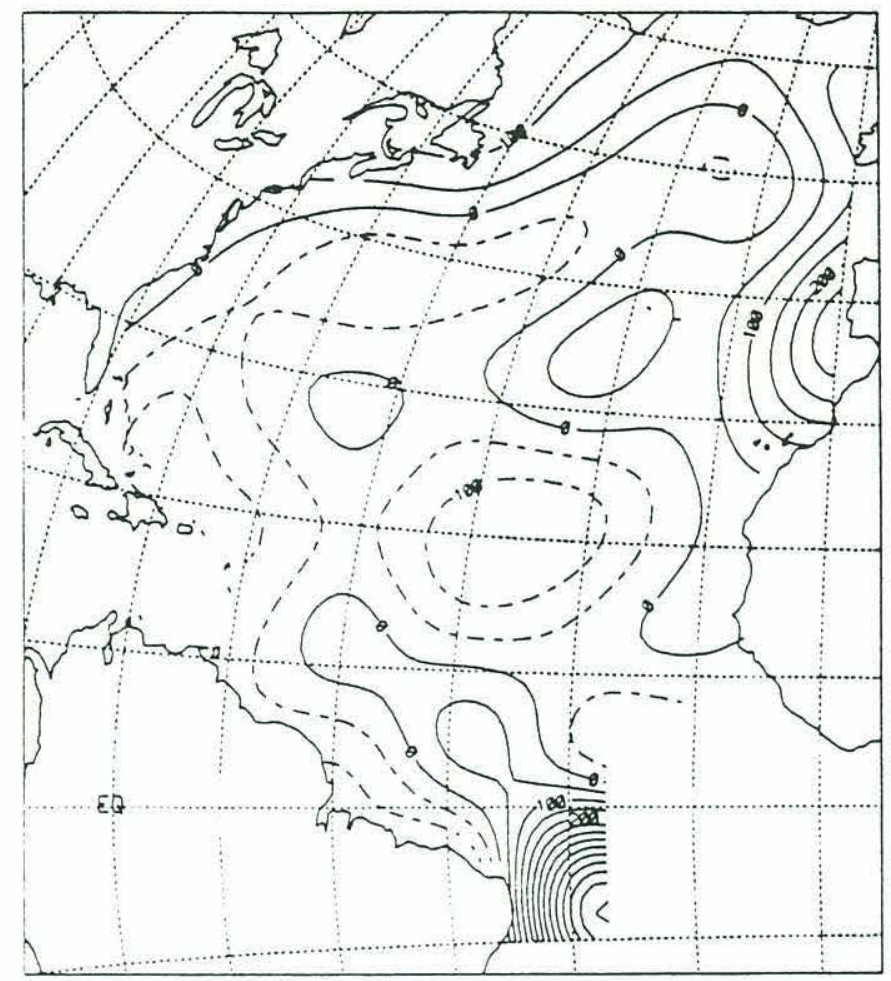

(f)

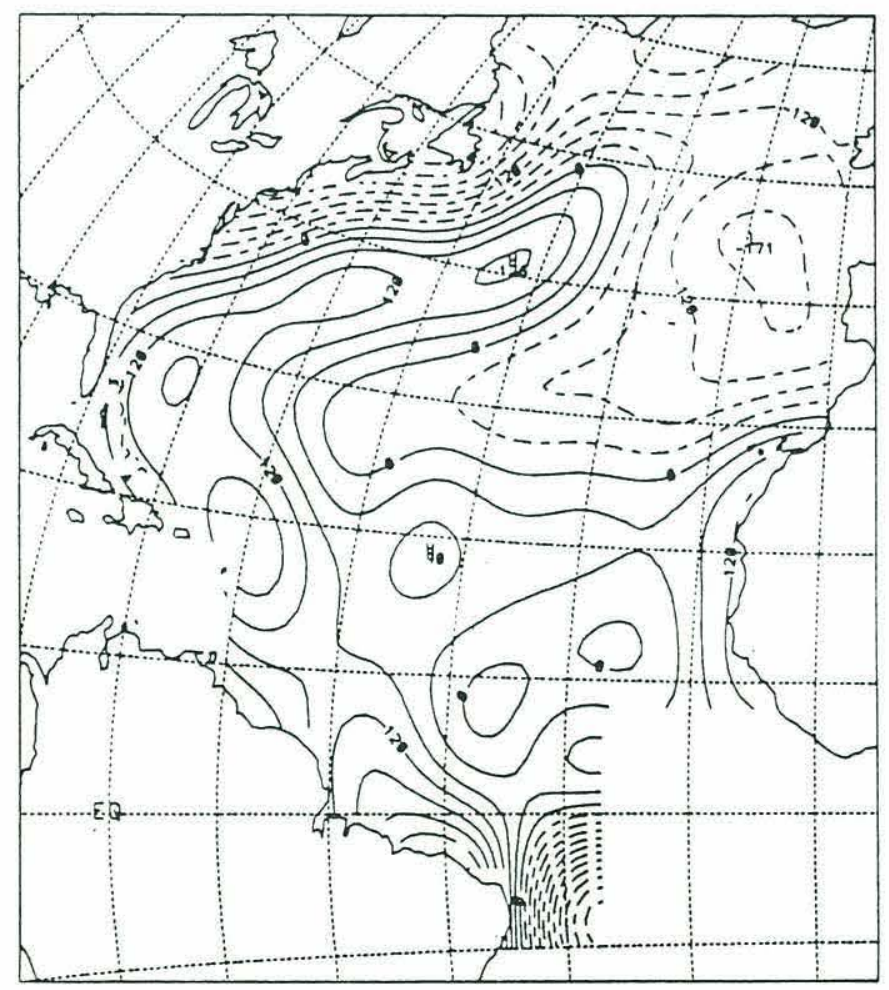

Figure 2-38. (continued) Mapped Expansion Coefficient $\left(\times 10^{-4}\right)$

(e) Temperature Mode 3 (f) Silicate Mode 3 
The vertical profile of the third mode (Figure 2-9) has a positive bulge at about standard depth 14 (1000 dbar) which is about the zero crossing of mode 2 . The third mode gives indications about property variations about depth 14 relative to the other depths. The third mode silicate (Figure 2-38f) shows a tongue of high values (higher silicate at depth 14) extending north in the western basin, which is indicative of the Antarctic Intermediate Water extending northward. However, the largest expansion coefficients are found in the tropics, south of the equator for both silicate and temperature. This explains why the third mode has such a large residual reduction in basin 3 (Section 5.1, Figure 2-13). This suggests that mode 3 is mainly tuned to the tropical variations, and as far as the rest of the ocean is concerned, the reduction may have been compromised. In fact the third mode temperature has little structure in the rest of the ocean (Figure 2-38e). Such extreme values south of the equator are also found in mode 4's expansion coefficients (not shown here).

\subsection{Correlations among Different Property Expansion Coefficients}

Salinity modal coefficients have extremely similar structure to temperature, while phosphate is similar to silicate. In fact the first mode expansion coefficients of different properties show high correlation (as defined by equation 1-11) between temperature and salinity and among the three nutrients (Table 2-6). Higher modes have similar high correlations among the six property distributions. The correlation coefficients for the sum of the first six modes, as defined by equations (1-12) and (1-14) of Chapter 1 , are summarized in Table 2-7. The values in Table $2-7$ are fairly similar to those values of the single section analysis in Chapter 1 (Table 1-5). There are two groups of high correlation; one is temperature and salinity and the other is the three nutrients.

As was done in Chapter 1, we can go one step further and determine how many degrees of freedom exists among the distribution of different properties. We calculate the modes of matrix $D$ as in equation 1-16 of the previous chapter, but including up 


\begin{tabular}{|c|c|c|c|c|c|c|}
\hline & $\mathrm{T}$ & $\mathrm{S}$ & $\mathrm{O}$ & $\mathrm{P}$ & $\mathrm{Si}$ & $\mathrm{N}$ \\
\hline $\mathrm{T}$ & 1.000 & 0.928 & -0.380 & -0.224 & -0.153 & -0.098 \\
$\mathrm{~S}$ & 0.928 & 1.000 & -0.141 & -0.460 & -0.377 & -0.340 \\
$\mathrm{O}$ & -0.380 & -0.141 & 1.000 & -0.738 & -0.811 & -0.830 \\
$\mathrm{P}$ & -0.224 & -0.460 & -0.738 & 1.000 & 0.934 & 0.952 \\
$\mathrm{Si}$ & -0.153 & -0.377 & -0.811 & 0.934 & 1.000 & 0.969 \\
$\mathrm{~N}$ & -0.098 & -0.340 & -0.830 & 0.952 & 0.969 & 1.000 \\
\hline
\end{tabular}

Table 2-6. First Mode Correlation Coefficient

Correlation coefficients are shown among the first vertical mode expansion coefficients of each property.

\begin{tabular}{|l|c|c|c|c|c|c|}
\hline & $\mathrm{T}$ & $\mathrm{S}$ & $\mathrm{O}$ & $\mathrm{P}$ & $\mathrm{Si}$ & $\mathrm{N}$ \\
\hline $\mathrm{T}$ & 1.000 & 0.817 & -0.102 & -0.437 & -0.383 & -0.408 \\
$\mathrm{~S}$ & 0.817 & 1.000 & -0.051 & -0.491 & -0.415 & -0.405 \\
$\mathrm{O}$ & -0.102 & -0.051 & 1.000 & -0.479 & -0.594 & -0.589 \\
$\mathrm{P}$ & -0.437 & -0.491 & -0.479 & 1.000 & 0.873 & 0.933 \\
$\mathrm{Si}$ & -0.383 & -0.415 & -0.594 & 0.873 & 1.000 & 0.919 \\
$\mathrm{~N}$ & -0.408 & -0.405 & -0.589 & 0.933 & 0.919 & 1.000 \\
\hline
\end{tabular}

Table 2-7. Correlation Coefficient among the sum of First 6 Modes 


\begin{tabular}{|l|c|c|c|c|c|c|}
\hline & 1 & 2 & 3 & 4 & 5 & 6 \\
\hline$e$ & $6.5 \mathrm{e}+2$ & $4.2 \mathrm{e}+2$ & $1.5 \mathrm{e}+2$ & $1.0 \mathrm{e}+2$ & $8.1 \mathrm{e}+1$ & $5.9 \mathrm{e}+1$ \\
$\%$ & 65.9 & 27.3 & 3.6 & 1.6 & 1.0 & 0.5 \\
\hline \multirow{4}{*}{$\underline{u}$} & 0.18 & 0.60 & -0.21 & -0.47 & -0.57 & 0.13 \\
& 0.25 & 0.58 & -0.42 & 0.43 & 0.46 & -0.17 \\
& 0.38 & -0.54 & -0.73 & 0.02 & -0.19 & 0.06 \\
& -0.45 & -0.03 & -0.35 & -0.56 & 0.30 & -0.51 \\
& -0.55 & 0.05 & -0.20 & 0.53 & -0.55 & -0.28 \\
& -0.50 & 0.06 & -0.30 & -0.01 & 0.21 & 0.78 \\
\hline
\end{tabular}

Table 2-8. Singular Value Decomposition of Expansion Coefficients Shown are the singular values and column singular vectors of matrix $D$. Also shown are each property mode's percentage variance to the overall variance of the 6 vertical mode reconstruction.

\begin{tabular}{|c|c|c|c|c|c|c|}
\hline modes & $\mathrm{T}$ & $\mathrm{S}$ & $\mathrm{O}$ & $\mathrm{P}$ & $\mathrm{Si}$ & $\mathrm{N}$ \\
\hline 1 & 15.7 & 27.5 & 49.6 & 91.8 & 95.2 & 95.4 \\
2 & 93.4 & 91.9 & 90.1 & 92.0 & 95.5 & 96.0 \\
3 & 94.6 & 96.4 & 99.8 & 94.9 & 96.2 & 97.8 \\
4 & 97.4 & 98.4 & 99.8 & 98.4 & 98.3 & 97.9 \\
5 & 99.9 & 99.9 & 99.9 & 99.0 & 99.8 & 98.1 \\
6 & 100.0 & 100.0 & 100.0 & 100.0 & 100.0 & 100.0 \\
\hline
\end{tabular}

Table 2-9. Cumulative Percentage Variance of Individual Property The percentage variance shown here is the percentage of the total modal variance of each property (sum of vertical mode 1 to mode 6 ) that the sum from the first property mode up to each particular property mode accounts for. 
to the sixth mode. The singular values and the modes are summarized in Table 2-8. Once again there are only two dominant degrees of freedom among the 6 variables, and the first 2 degrees of freedom account for $93 \%$ of the total horizontal property variability. Looking at each separate property, these 2 modes account for nearly the same percentage variance (more than 90\%) for all six properties (Table 2-9).

\subsection{Modal Model}

The mapped coefficients define a model ocean. We may call this the modal-model ocean. The original hydrographic variables (temperature, salinity, oxygen, phosphate, silicate, and nitrate) can be calculated at every grid point from the mapped coefficients. Derived quantities, such as density, can be calculated from these reconstructed hydrographic variables. As an example, Figure 2-39 shows the dynamic topography of the sea surface referenced to 3000 dbar. Figure 2-39 displays a reasonable pattern of a subtropical dynamic high reminiscent of the anticyclonic subtropical gyre with steep gradients to the north corresponding to the Gulf Stream. Examination of this modal-model will be the topic of the next chapter.

\section{Summary}

Just as in the case for a single hydrographic section in Chapter 1, we have found that there is a limited number of degrees of freedom that would describe the North Atlantic Ocean. Many of the results found in Chapter 1 still apply in principal for the entire ocean. Six primary modes account for almost all of the major hydrographic variability as well as the circulation. In the spatial distribution of the six properties analyzed (temperature, salinity, oxygen, phosphate, silicate, and nitrate), there are only two principal degrees of freedom. The reduction in vertical and between different properties combined would give a reduction factor of $17.5(6 / 35 \times 2 / 6)$.

On the other hand some residuals show regional dependence. Residuals, namely the differences between original data and modal reconstructions, are larger in some 


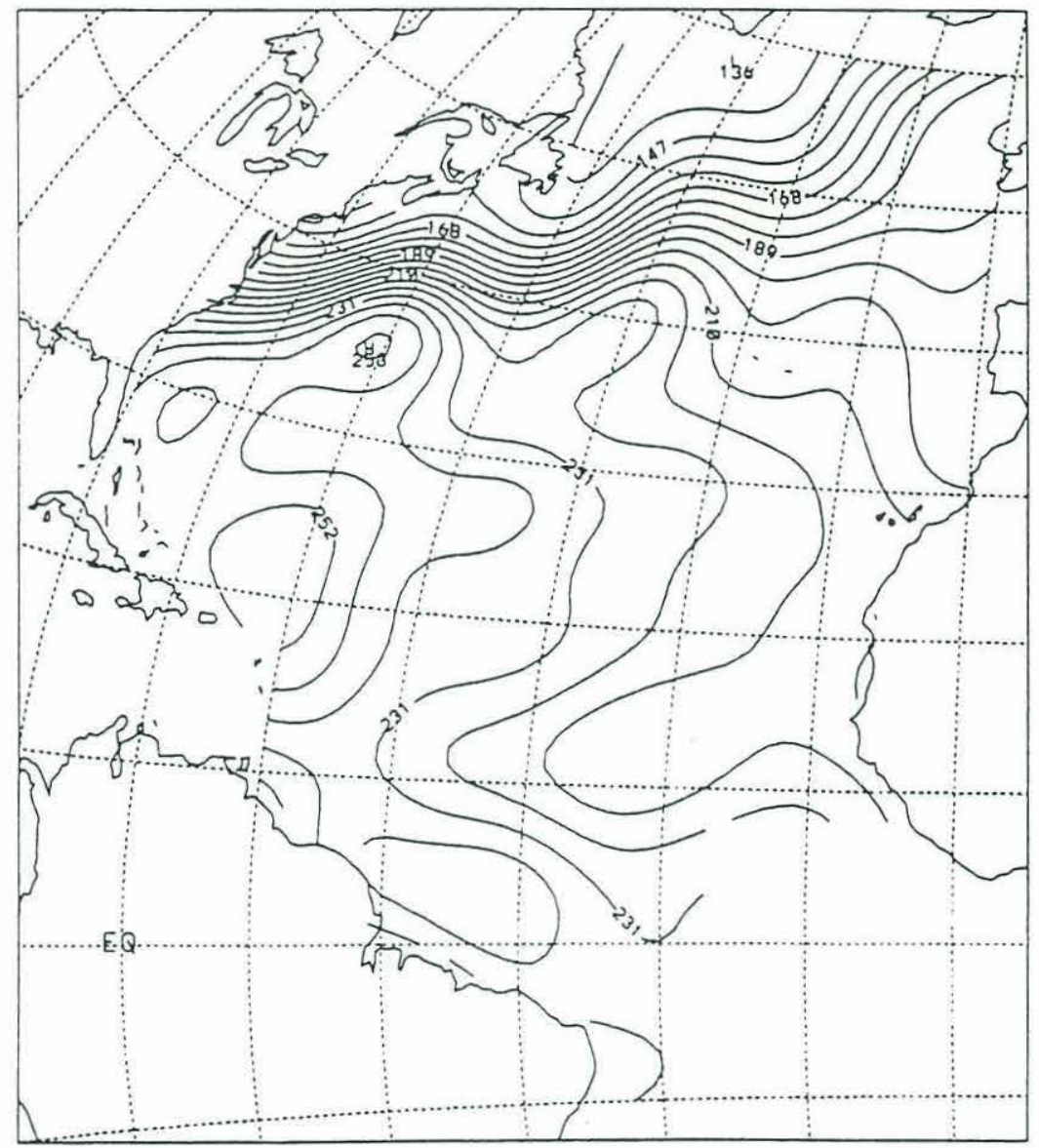

Figure 2-39. Sea Surface Dynamic Height Anomaly of the Modal-Model Dynamic height $\left(\times 10^{-2}\right.$ dyn $\left.\mathrm{m}\right)$ is based on hydrographic variables reconstructed from 6 modes which expansion coefficients are objectively mapped on every degree latitude and longitude. The dynamic height values are referenced to $3000 \mathrm{dbar}$. 
regions than in others. Examples of such regions are inside the core of the northward spreading Antarctic Bottom Water along the western end of the equatorial stations, or the southward spreading Labrador Sea and Greenland Sea waters which flank the base of the continental shelf in the area of the Grand Banks. These anomalies point to interesting regions where there are physics the modes do not completely resolve.

In a low mode reconstruction (even after 6 modes), residuals on the deepest depth level hardly changes from the original data variance. This result owes to a lack of resolution at this deepest level. Similar to inverse problems, we can compute the resolution matrix, which tells us which depths are being resolved. It is found that no depth is resolved independently of another depth, and so the modal reconstruction is truly an approximation to the data in a vertically integrated sense and not at single depths. This finite depth resolution can result in a Gibbs-like phenomena in spectral analysis, when the data has short vertical scale anomaly, such as those found in the salinity profile near the Mediterranean outflow. A simple vertical average was shown to be effective in reducing some of the spurious structures.

Surface dynamic topography referenced to a deep level was found to be well reproduced by a low mode model. In fact, dominant variations such as the Gulf Stream or cold- and warm-core rings are adequately reproduced by the first two modes. This agreement strongly contrasts with the lack of such agreement for surface properties such as temperature.

Reproduction of surface topography by the modal reconstruction seems to imply that measurements of surface topography from such observations as an altimeter can provide information about subsurface variations due to the gravest modes. Lack of such agreement for surface temperature indicates that measurement of sea surface temperature will not allow a simple statistical prediction of subsurface hydrographic structure. 
The modal expansion coefficients show spatial coherence. Their patterns reflect spatial distribution of different water masses and hydrographic structural variation associated with current systems. However, as each mode does not resolve a particular depth and as hydrographic variation is described by the sum of modes, there is not a simple relation between a particular water mass and any one of the modes. The modes provide the most efficient description of the structure of the ocean, but is quite a different description than traditional water mass analysis.

\section{Conclusion}

We have demonstrated that the North Atlantic Ocean can be adequately described using a small set of modes to within a measure of noise. We have shown that there is a redundancy in the traditional form of data. Apparently, there is a greater volume of numbers involved than necessary to both describe the general hydrographic structure and to study the general circulation of the ocean. Six vertical degrees of freedom among the 35 standard depths, and 2 degrees of freedom among the 6 properties suffice. The limited degrees of freedom reveals the underlying simplicity of the hydrographic structure of the North Atlantic Ocean.

The number of modes deemed significant were chosen by comparing the original data with modal reconstructions of varying degrees of freedom. Modes are added until the residuals (i.e. differences between data and modal reconstruction) become smaller than a measure of goodness of fit. We must emphasize, however, that the purpose of the modal decomposition is not to reproduce the data itself. We can always reproduce the data better and better by adding more and more modes into the reconstruction. Conversely, we have a model ocean of varying complexity by varying the number of significant modes. The number of modes and how well the sum of modes reproduce the original data is a tradeoff. The more the modes the smaller the residual. 
On the other hand, a low mode reconstruction will always be meaningful even if there are some residuals, just as filtered time series are still significant. For example, a reconstruction by the first 2 modes will inevitably miss some hydrographic feature, but the gross nature of the ocean structure will still be present, and this may be quite adequate for some purposes. As far as the surface dynamic topography is concerned, we saw that these first 2 modes account for most of the significant variations.

The primary objective of this study was to identify if there are a limited degrees of freedom in ocean structure. We determined what these elements are and examined some of their uses. Determining the exact number of significant modes is complex, and depends on what exactly "significant" means. If this number is too large, then the economization goal of modal decomposition will be defeated. However, as we saw, this number is small in most reasonable circumstances, and the modes are ordered in exponentially decreasing importance. To appreciate the significance of the modal analysis, lets put the results into perspective.

One of the primary goals in oceanography has always been to obtain as complete, comprehensive, and meaningful description of the entire ocean as possible. For this purpose, various hydrographic observations are combined into a single picture. Each observation, say a hydrographic section, is synoptic in the sense that the measurements were taken over a limited area at a particular time. The significance of such synoptic data to the overall state of the ocean is limited at best and often difficult to interpret. We may observe a certain Gulf Stream path at one time and a different path at another time (e.g. Fofonoff, 1981). Or, the Gulf Stream mass transport may be different from one time to another (Fu et al., 1987). Seasonality and other temporal variability are essential in many parts of the ocean. How should one treat two observations at the same geographic location but one with a Gulf Stream ring and another without?

An alternative description of the ocean is to consider a mean ocean, where temporal variabilities are averaged out. One example is the Levitus' climatological atlas of the 
world ocean (Levitus, 1982). However, fluxes of various properties for such a mean ocean will not necessarily be the same as fluxes of any synoptic observation. This is so because flux is a nonlinear product of the properties. Thus the physical significance of such a mean ocean is not easy to understand. Additionally, it is apparent that such a mean state is never realized in the real ocean, which is always full of mesoscale eddies.

Ideally we would like to have a synoptic data set that covers the entire ocean at some common time. However, in light of available resources (tools, methods, man power, money) such global synoptic data set of the ocean is far from becoming a reality (unlike the synoptic observation network for meteorology).

An alternative approach may be to consider a "typical" ocean. A typical ocean is an ocean model with realistic hydrographic structure and with the mean property fluxes. It is a model ocean that might be physically realized. Or to be more accurate, it is a model that is indistinguishable from what we know of the possible synoptic state of the ocean. It must have the known essential characteristics of the ocean. As a first step towards constructing such a model, we must quantify such essential characteristics.

The hydrographic modes we have analyzed offer one example of quantification of such characteristics. The series of modes identify hydrographic variations in decreasing order of significance. These modes provide efficient building blocks of such a "typical" ocean. We might regard this as a "first order model" of the hydrographic structure of the ocean. We need only to use those essential modes to construct this ocean model rather than to analyze each standard depth separately.

The step to construct a model ocean is then to determine the expansion coefficients of each mode over the entire horizontal extent of the ocean. One such example was given in Section 8, where we interpolated the expansion coefficients of the raw data onto a regular grid. However, we have filtered out short horizontal scale variability from this model, and so the resulting model becomes qualitatively similar to a time 
mean ocean model. Whether a modal model of limited degrees of freedom is indeed realistic at all will be examined in Chapter 3.

The modal analysis has revealed the simplicity of the ocean structure, which in turn implies that analyses of general circulation could be done easily as well. For example consider a geostrophic inverse problem as in Wunsch (1978). Among the six properties, there are only two independent ones. Among the 35 depths, we only need to examine six depths or six layers rather than having constraints on each depth.

In light of the massive data to become available from the upcoming World Ocean Circulation Experiment, the results of the present analysis are significant. Our findings provide insight into how we could proceed most efficiently in the analysis of the global hydrographic data. The hydrographic modes allow reduction of the volume of numbers to describe the ocean without losing essential information.

The results of the analyses of this chapter are still not definitive. We have seen regions where the modes have slight biases, which in turn indicate regional physical processes. A different normalization of the data or a different definition of modes such as abyssal modes or using density coordinates might be better in resolving these regional physics. However, the issue of how to best analyze the ocean general circulation is an important problem, and the analyses presented here are in the right direction towards advancing our knowledge of the ocean. 


\section{CHAPTER 3 \\ Determining Circulation over the Mediterranean Tongue}

\section{Introduction}

In Chapter 2, we have found that the North Atlantic Ocean can be described adequately by a small set of modes. An ocean model was then constructed by this set of modes by interpolating the expansion coefficients of the raw data onto a regular grid (Section 8 in Chapter 2). The grid is at every degree in both latitude and longitude, and covers virtually the entire North Atlantic Ocean. In this chapter, we examine a portion of this model ocean to determine the absolute circulation. The purpose of this study is three-fold.

One of the objectives is to demonstrate that a model ocean with limited degrees of freedom is physically consistent, and thus useful in the quantitative analysis of the ocean circulation. The prime question is whether the dominant structures in the low mode model retains the important features of the hydrographic structures from which ocean circulation can be determined.

Recently two methods have been put forth to estimate the absolute velocity from density data alone. One is the box model inverse of Wunsch (1977) and the other is the $\beta$ spiral of Stommel and Schott (1977). Both of these methods are a linear regression for the geostrophic reference velocity based on kinematic and dynamic constraints. While the methods have similar constraints, the essential difference is the way these constraints are applied. One version of the box model (Wunsch, 1978) is based on integral constraints imposed on a series of layers, often bounded by isopycnal surfaces, which delineate different water masses. The $\beta$ spiral on the other hand is a local balance, that uses the differential form of constraints, applied to areas where substantial hydrographic data exists to compute the three dimensional spatial gradients of property (Schott and Stommel, 1978). In this section we shall compare these two methods and 
examine how best one should go about in studying circulation from density data alone when given a smoothed but densely spaced data as the one we have now.

The area we choose to analyze is a square region, $12^{\circ}$ wide on each side, located towards the eastern edge of the wind-driven anticyclonic subtropical gyre, situated over the Mediterranean Water tongue (Figure 3-1). This $12^{\circ}$ region is chosen as it has the minimum mapping error estimate (Figure 3-2), and is also situated over a relatively flat bottom topography. The area is interesting for examining absolute circulation in its own right. The presence of the Mediterranean tongue has been a subject of many studies. Whether the westward extending tongue is entirely an advective feature flowing out from the Strait of Gibraltar, or primarily a diffusive structure spreading west is not clear (Richardson and Mooney, 1975). The dynamical effect the salt tongue has on the general circulation is also an interesting problem (Arhan, 1987; Tziperman, 1987b). The observation that the Mediterranean tongue extends westward over much of the North Atlantic led Worthington (1976) to conclude that the anticyclonic wind driven circulation is confined to the western basin of the North Atlantic Ocean. To close this circulation, Worthington took the drastic step of abandoning geostrophy, one of the reasons that prompted reopening of the subject of estimating absolute circulation from density data.

This chapter is organized into seven sections. In Section 2, we briefly describe the temperature and salinity field of this $12^{\circ}$ square. We also examine the geostrophic circulation. Comparison of the hydrographic structure is made with climatological data to examine how reasonable our model is. The inversion is presented in sections 3 to 5. Results from applying one version of the $\beta$-spiral method is discussed in Section 3, while the box model is studied in Section 4. Both models use potential density conservation as the constraining equation. A hybrid method that has both an integral and a differential constraint is presented in Section 5. Comparison of the results to 


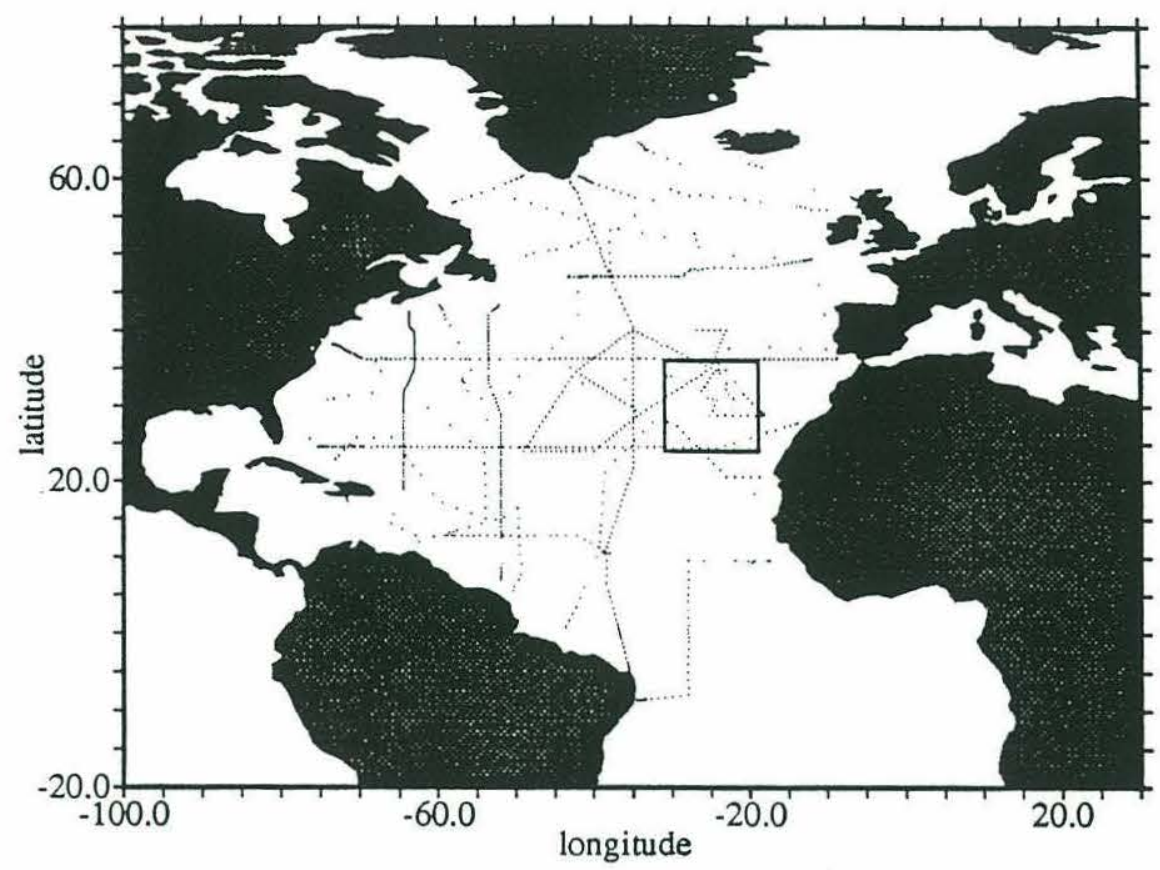

Figure 3-1. Location of $12^{\circ}$ Square.

The $12^{\circ}$ square is outlined by a thick line. The dots on the plot are station locations used in modal calculations (Figure 2-7).

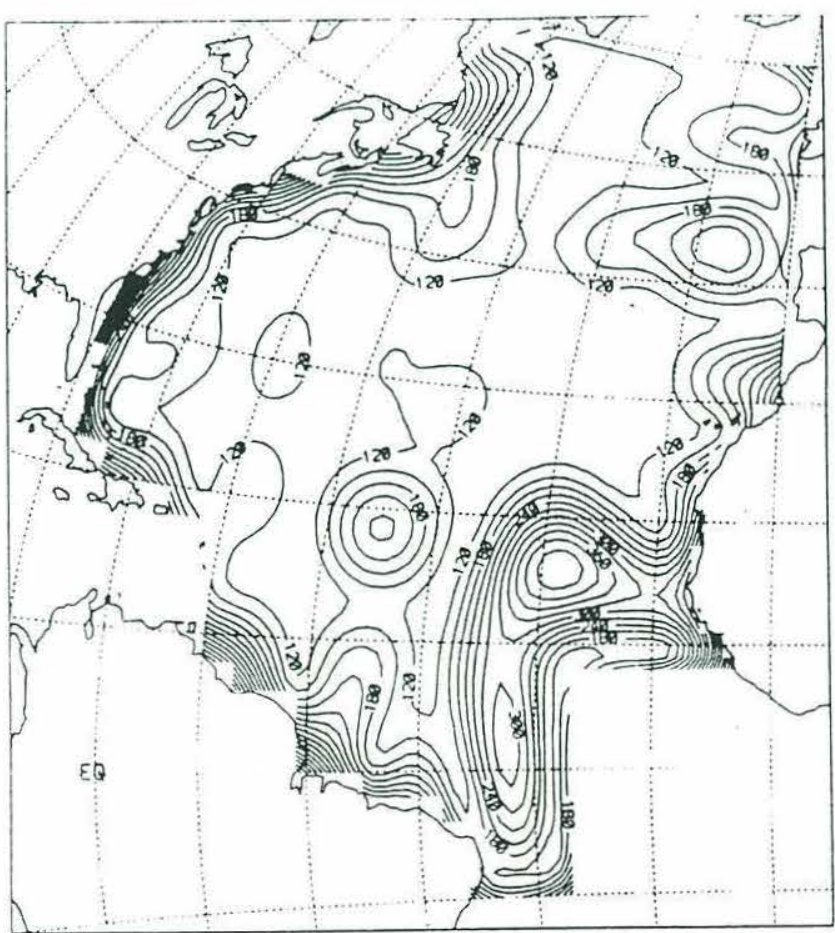

Figure 3-2. Error Map of Mode 1 Expansion Coefficients $\left(\times 10^{-5}\right)$. 
previous studies concerning the circulation in this region is given in Section 6. The chapter ends with a summary and conclusion presented in Section 7.

\section{General Hydrographic Structure}

The $12^{\circ}$ box is located east of the Mid-Atlantic ridge towards the eastern edge of the subtropical gyre (Figure 3-1). Figure 3-3 shows temperature at 500 dbar (standard depth 9). Temperature is higher towards the west and center of this $12^{\circ}$ square, which is consonant with the deepening of the thermocline westward towards the center of the subtropical gyre. It is interesting to compare our mapped model with the Levitus' (1982) atlas (Figure 3-4). Despite the differences in the scheme of analysis and the amount and time scale spanned by the raw data, the two estimates appear consistent with each other in the sense that we can not distinguish which is a better representation of the real ocean.

At deeper levels, the influence of the Mediterranean outflow becomes evident. In fact this $12^{\circ}$ square straddles the axis of the Mediterranean salt tongue. Figure 3-5 shows the temperature and salinity contours at $1200 \mathrm{dbar}$, close to the maximum salinity anomaly associated with the Mediterranean Water (Hogg, 1987). The influence of the Mediterranean is seen as the warmer and more saline water emanating from the northeast corner towards the southwest.

Figure $3-6 \mathrm{a}$ is salinity contoured vertically along $25^{\circ} \mathrm{W}$. The influence of the Mediterranean Water is evident about $1000 \mathrm{dbar}$ as the halostad, seen as the widening of the intervals between isohalines. The depth of the halostad deepens to the south indicating that the Mediterranean tongue axis shifts southward at deeper depths (Arhan, 1987). A salinity section along $25.5^{\circ} \mathrm{W}$ from the Levitus' atlas (Figure 3-6b) is fairly similar to Figure 3-6a. The structure of the halostad are consistent between the two data sets. In both models, the near surface stratification is stronger at the southern end and decreases northward. 


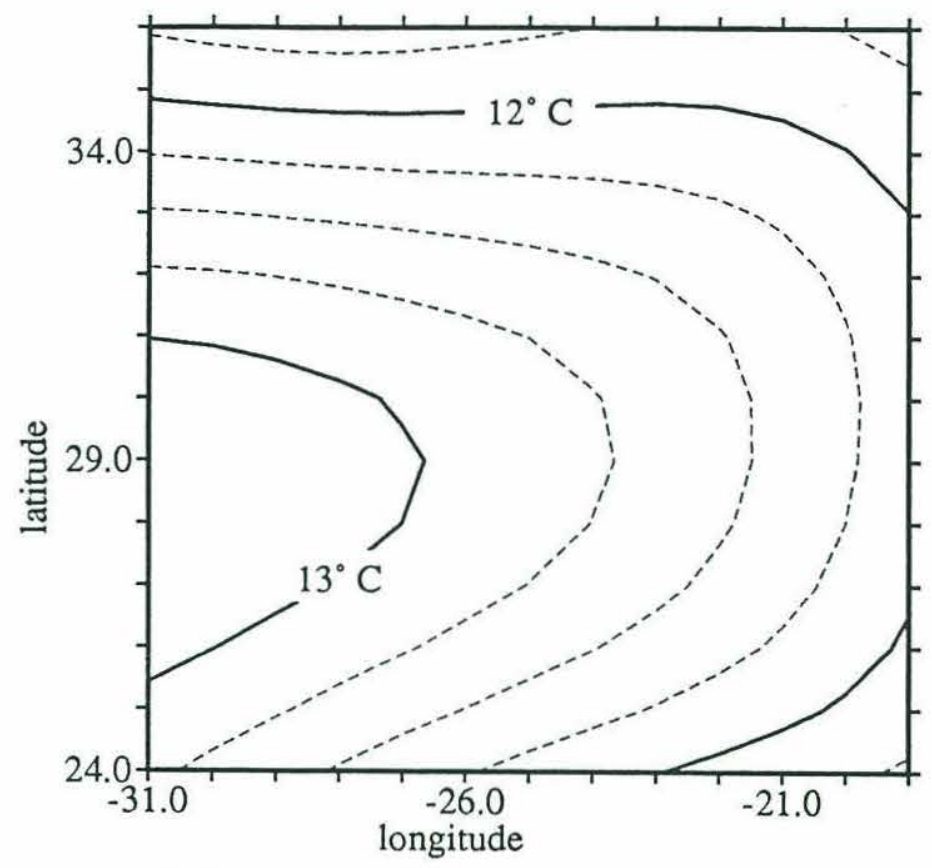

Figure 3-3. Modal Model Temperature on 500 dbar (standard depth 9)

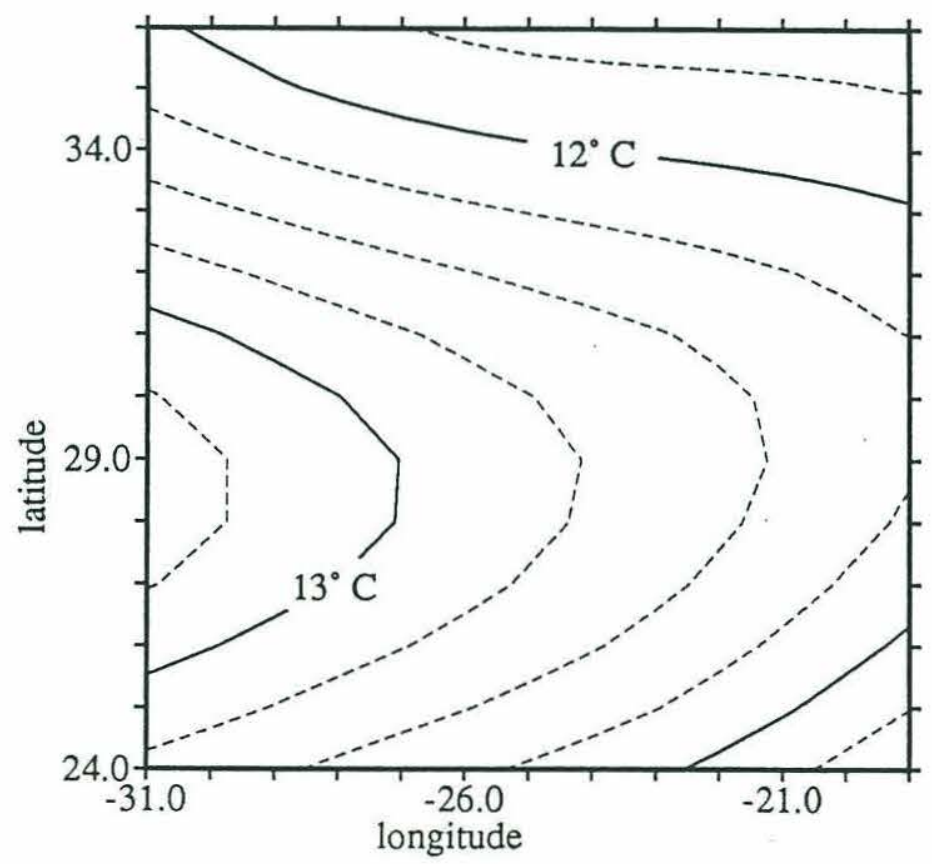

Figure 3-4. Levitus' Atlas Temperature on $500 \mathrm{~m}$ 

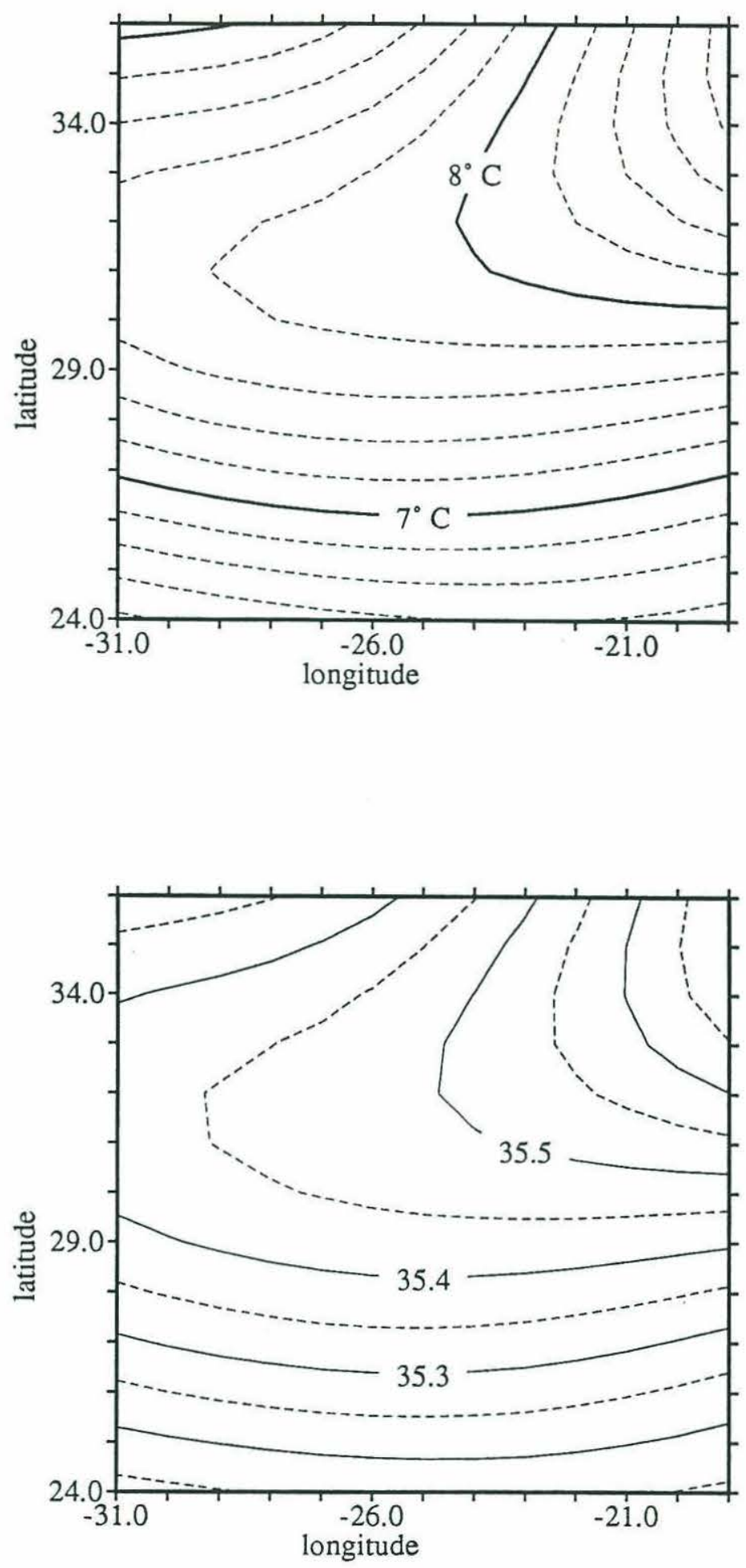

Figure 3-5. Temperature and Salinity on 1200 dbar Surface 

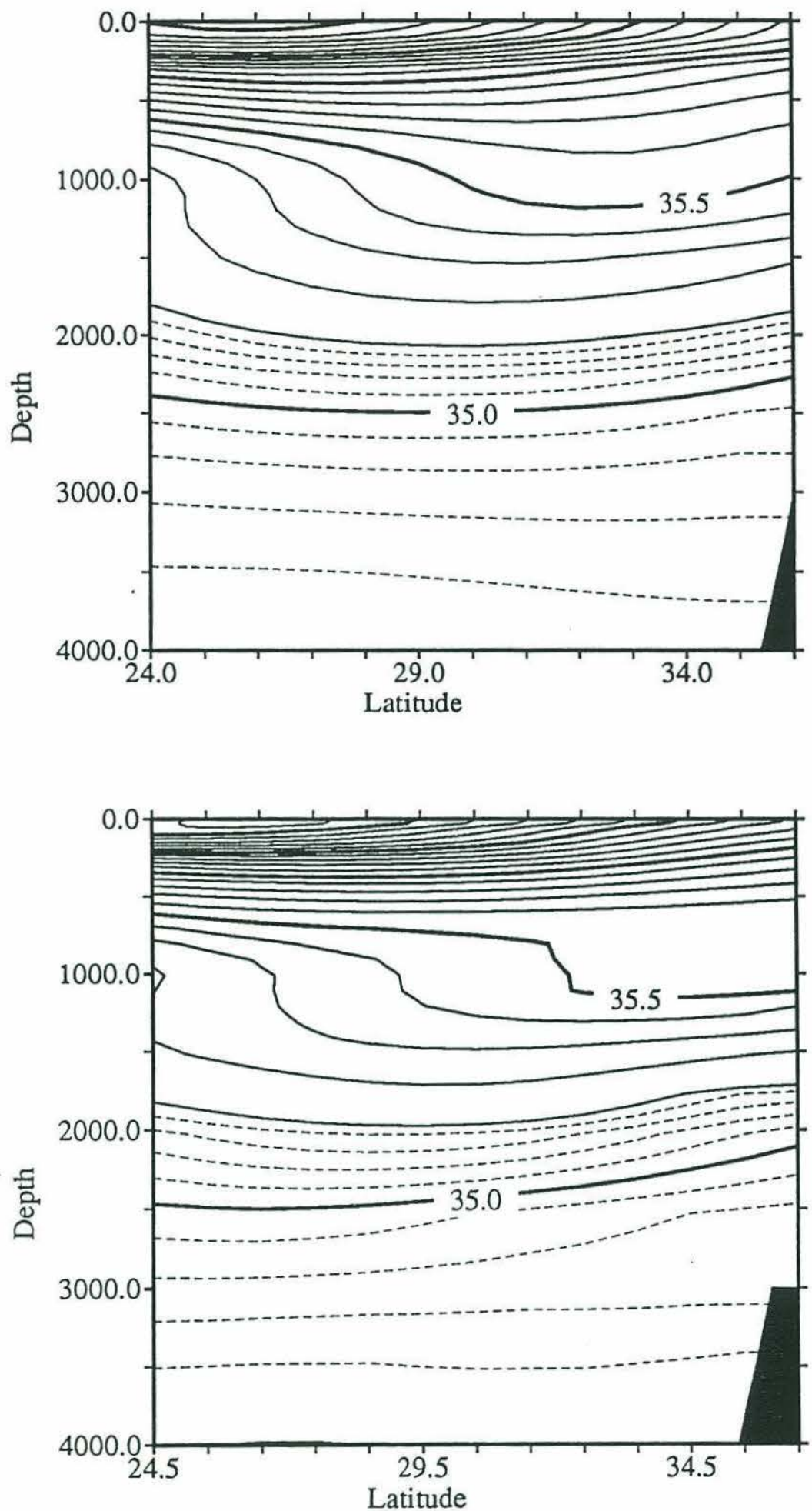

Figure 3-6. Salinity Contours along $25^{\circ} \mathrm{W}$

(a) Modal Model (b) Levitus Atlas (along $25.5^{\circ} \mathrm{W}$ ) 

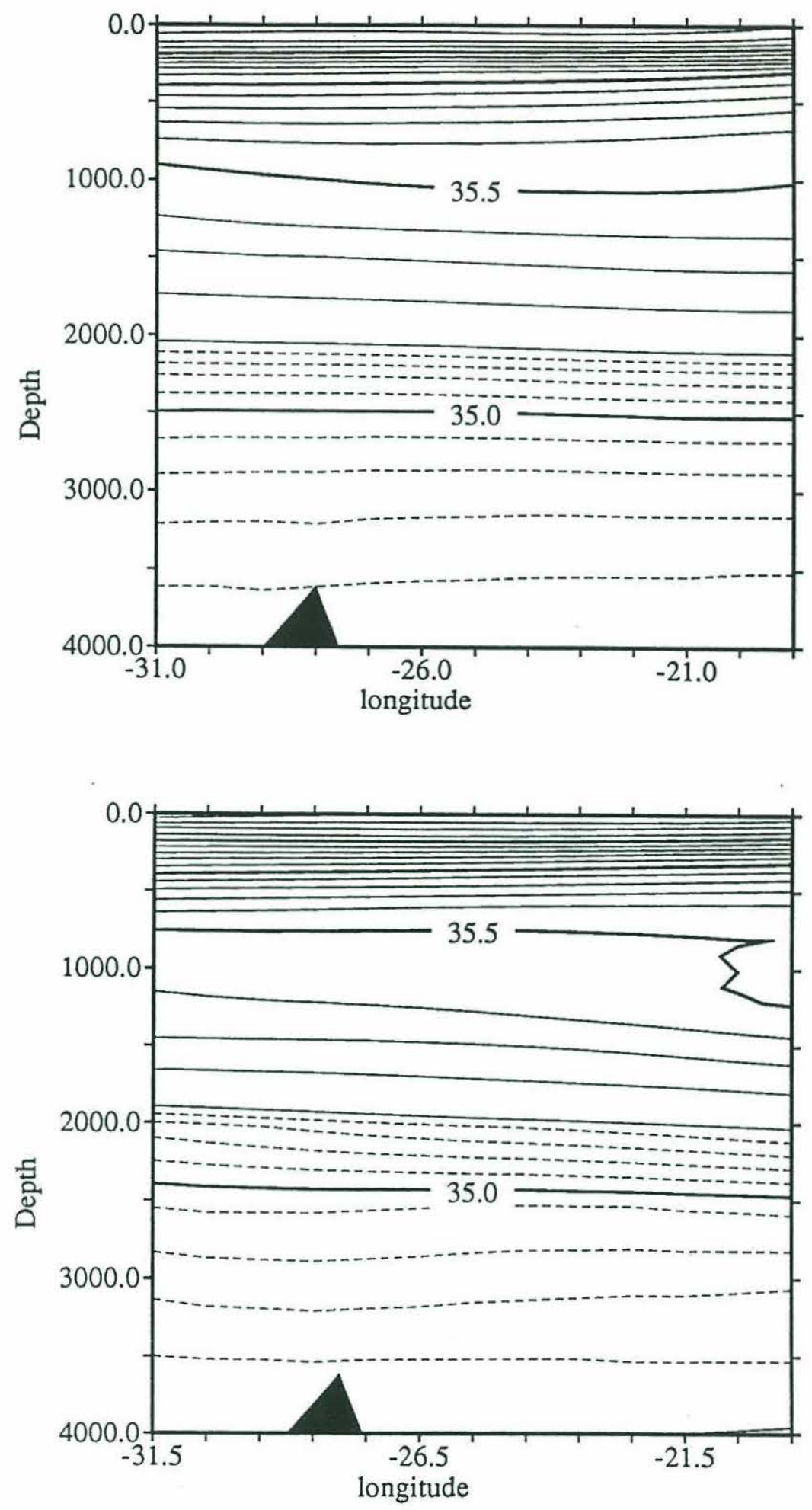

Figure 3-7. Salinity Contours along $30^{\circ} \mathrm{N}$

(a) Modal Model (b) Levitus Atlas (along $30.5^{\circ} \mathrm{N}$ ) 
Figure 3-7 shows the east-west salinity section along $30^{\circ} \mathrm{N}$ for the modal model and the Levitus' atlas. The agreement between the two is again quite satisfactory. Compared to the meridional section along $25^{\circ} \mathrm{W}$ (Figure 3-6), the structure of this zonal section is relatively simple. Nevertheless we see the isohalines shoaling to the east at shallower depths $(<700 \mathrm{dbar})$, consistent with the "bowl" shape halocline of the subtropical gyre. Below the halocline (> 1000 dbar), the thickness of the halostad associated with the Mediterranean Water increases eastward.

Lastly we examine the geostrophic velocity of the mapped field. Geostrophic velocity is calculated by centered differencing over $2^{\circ}$ latitudinally and longitudinally. As an example, the velocities at $500 \mathrm{dbar}$ and $1200 \mathrm{dbar}$ relative to $2000 \mathrm{dbar}$ are plotted in Figure 3-8. At 500 dbar the dominant flow is the anticyclonic circulation of the subtropical gyre. The velocities are on the order of $1 \mathrm{~cm} / \mathrm{sec}$ with the circulation being primarily flowing into the region from the northwest, turning clockwise and flowing out towards the southwest.

At 1200 dbar the anticyclonic flow is not evident anymore, and the flow is primarily to the east or northeast towards the southeast half of the region with magnitudes on the order of several $\mathrm{mm} / \mathrm{sec}$. Interestingly there is a slight hint of a cyclonic circulation in the region with westward flow in the northern half, thus circulating around the salinity anomaly of the Mediterranean Water (Figure 3-5). This result is in contrast with a Wüstian method of circulation analysis (i.e. core layer method, Wüst, 1935), in which the direction of spread would be estimated to be along the axis of anomaly. Determining the absolute velocities is the subject of the sections to follow.

The geostrophic velocity hodographs, or the $\beta$-spirals, referenced to $2000 \mathrm{dbar}$ are shown in Figure $3-9 a$ at every $2^{\circ}$. The angle of these velocities relative to the local gradient of tracers form the basis of the $\beta$-spiral method. The upper levels show the anticyclonic circulation of the subtropical gyre. While the eastward flows in the northern half of the region have nearly the same direction with depth, the return flow 
a.

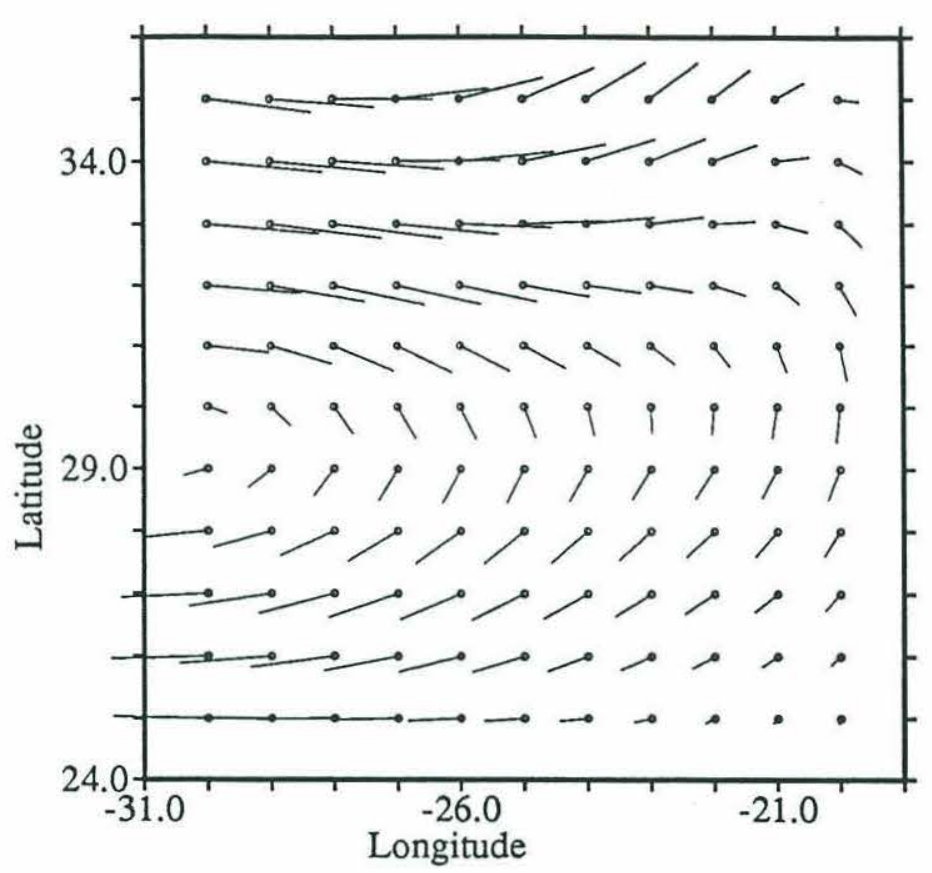

b.

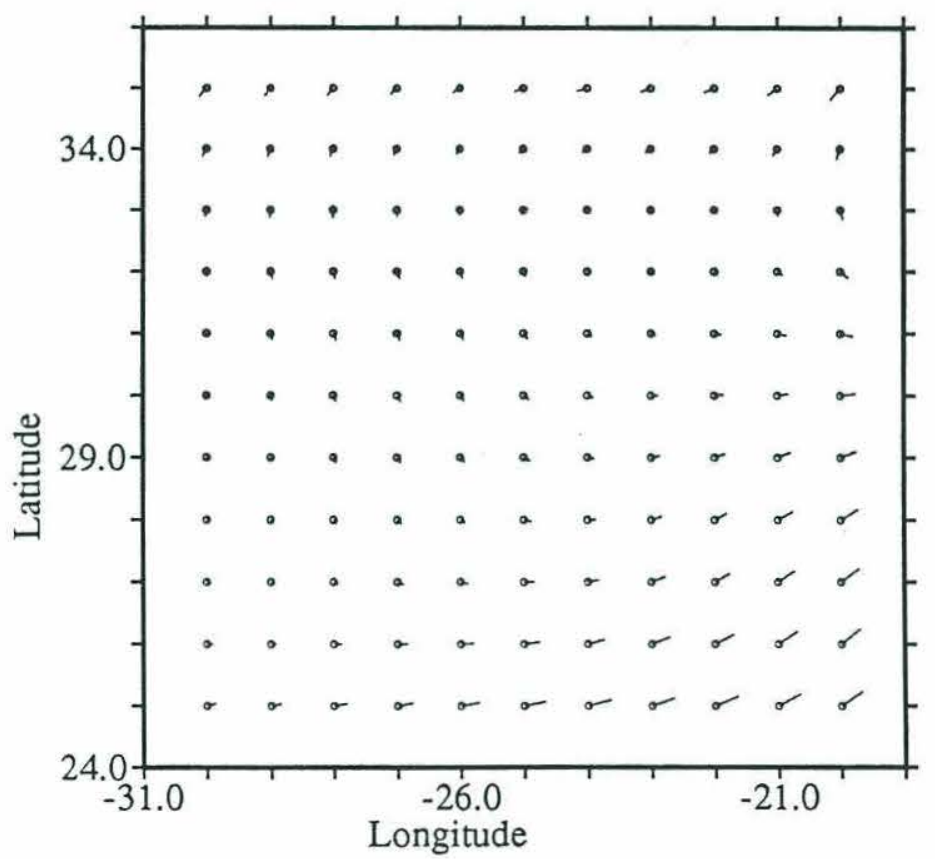

Figure 3-8. Relative (ref. 2000 dbar) Geostrophic Velocities The scale is such that $1(\mathrm{~cm} / \mathrm{sec})$ equals $1^{\circ}$.

(a) 500 dbar (b) 1200 dbar 

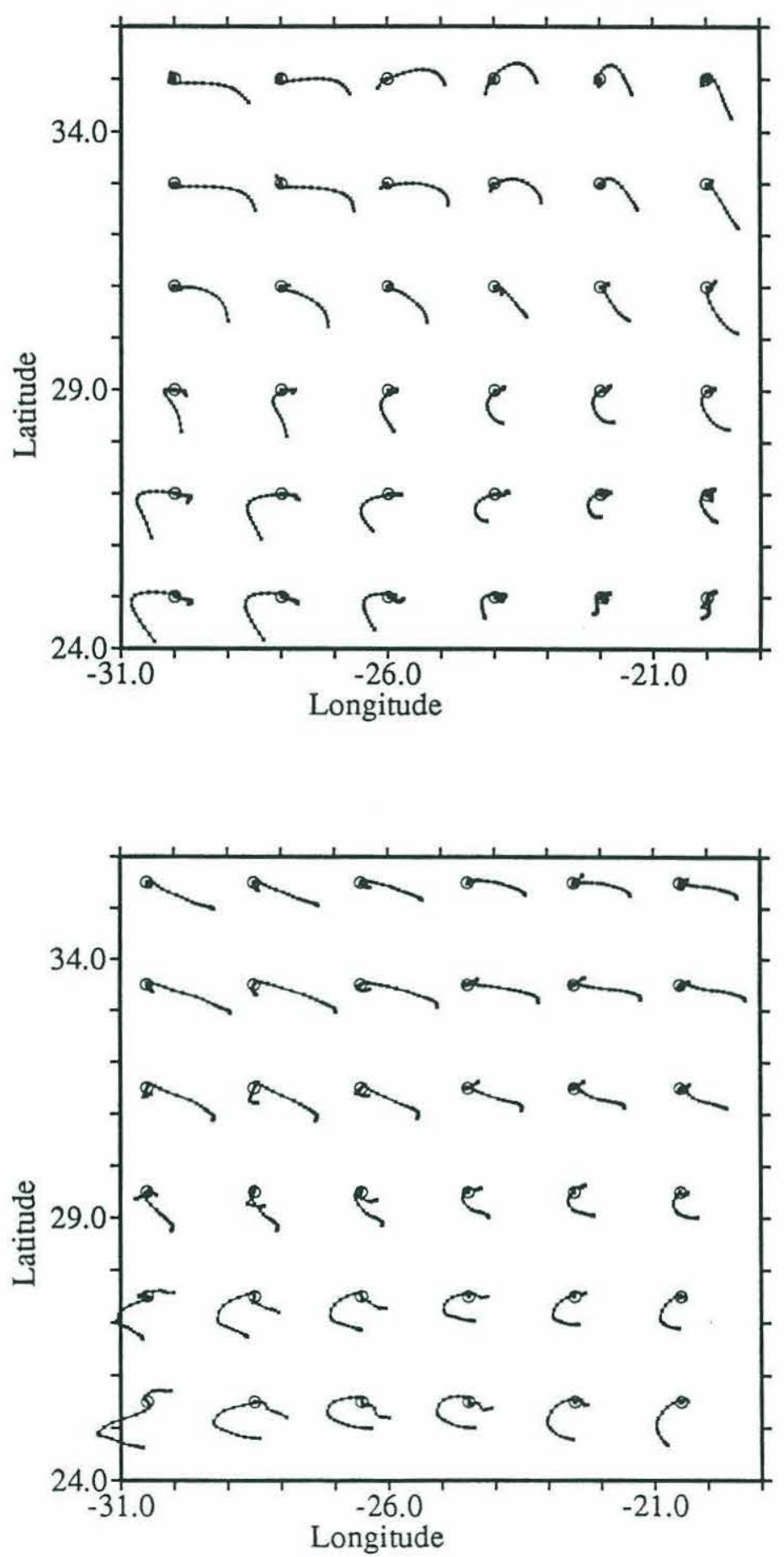

Figure 3-9. Geostrophic Velocity (2000 dbar ref.) Hodographs The scale is such that $1(\mathrm{~cm} / \mathrm{sec})$ equals $1^{\circ}$.

(a) Modal Model (b) Levitus 
in the southern half change their direction clockwise with depth. This is consistent with the notion that the southern extent of the subtropical gyre recedes to the north with increasing depth. For comparison, we again show the corresponding $\beta$-spiral based on the Levitus' atlas in Figure 3-9b. The velocity hodographs are fairly consistent with each other. The magnitude of the flow is similar as well as their direction. The hodographs have a similar "C" shape towards the south and little directional change at north. Interestingly, the surface eastward flow turns westward around $30^{\circ} \mathrm{N}$ in both data sets. Some differences between Figures $3-9 a$ and $3-9 b$ are evident towards the northeast corner where the modal reconstruction has a north-south component, and the southeast corner where the modes result in smaller velocities.

\section{Estimate of Absolute Velocity Using the $\beta$-spiral Method.}

\subsection{Review of Method}

The $\beta$-spiral method is a linear regression for obtaining reference level velocities using local kinematic and dynamic constraints. The method is local in the sense that it uses a differential form of constraints, and the velocities are determined at a point independent of the surrounding velocities. The essential idea behind the method is as follows. There are properties which are constant along streamlines. Then, in the absence of mixing, the absolute velocities must lie tangent to these surfaces of conserved tracers. If there are two or more tracers which distribution are not coincidental, the surfaces defined by each tracer will intersect as a single curve. This curve defines the direction of the flow, thus constraining the values the reference velocities must take.

There have been several formulations of the $\beta$-spiral method which differ in the ways the regression is done, how mixing is treated, what tracers are used, and how vertical velocity is evaluated. Here we shall briefly summarize some of the those methods with emphasis on the last 2 points. 
Stommel and Schott (1977), Schott and Stommel (1978), and Schott and Zantopp (1979), have used density as the tracer. Instead of using another tracer, they explicitly use the linear vorticity balance,

$$
\beta v=f w_{z},
$$

to eliminate vertical velocity, $w$ from the equations. The resulting equation has the 2 . components of the horizontal reference velocity as the unknowns and has coefficients involving second order derivatives of the isopycnal depths.

Behringer (1979), Behringer and Stommel (1980), and Needler (1985) have used spatial gradients of density and potential vorticity $\left(f \rho_{z}\right)$ to define the direction of the horizontal component of the stream line. The resulting equation is again for the horizontal reference level velocities $\left(u_{0}, v_{0}\right)$, and the coefficients of the regression equations involve vertical gradients of the horizontal derivatives of density.

Finally, Olbers et al. (1985) use density as the tracer, but by explicitly integrating the linear vorticity balance vertically and thus introducing an unknown reference level vertical velocity $\left(w_{0}\right)$, they arrive at an equation that only involves first order differentials, but at the expense of the additional unknown, $w_{0}$. We shall use a variation of Olbers et al.'s (1985) formula to estimate the absolute velocities, derived as follows:

The horizontal velocities are estimated from the thermal wind relation as,

$$
\begin{aligned}
& u=u_{0}-\frac{1}{f} D_{y} \equiv u_{0}+u^{\prime}, \\
& v=v_{0}+\frac{1}{f} D_{x} \equiv v_{0}+v^{\prime},
\end{aligned}
$$

where $D$ is dynamic height referenced to the reference level at $2000 \mathrm{dbar}$,

$$
D=\int_{2000}^{p} \frac{d p}{\rho} .
$$


The vertical velocity is obtained by vertically integrating the linear vorticity balance, equation (3-1);

$$
\begin{aligned}
w & =w_{0}+\frac{\beta}{f} \int_{z_{0}}^{z} v d z \\
& =w_{0}+\frac{\beta}{f}\left(z-z_{0}\right) v_{0}+\frac{\beta}{f} \int_{z_{0}}^{z} v^{\prime} d z
\end{aligned}
$$

The constraint that density be conserved following a water parcel is,

$$
u \sigma_{x}+v \sigma_{y}+w \sigma_{z}=0
$$

Substituting $u, v$, (equation 3-2), and $w$ (equation 3-4) into equation (3-5), we obtain,

$$
u_{0} \sigma_{x}+v_{0}\left(\sigma_{y}+\sigma_{z} \frac{\beta}{f}\left(z-z_{0}\right)\right)+w_{0} \sigma_{z}=-\left\{u^{\prime} \sigma_{x}+v^{\prime} \sigma_{y}+\sigma_{z} \frac{\beta}{f} \int_{z_{0}}^{z} v^{\prime} d z\right\}
$$

This is equivalent to equation (33) of Olbers et al. (1985) except we have neglected the non-adiabatic terms in the density equation. We assume no cross isopycnal exchange. Equation (3-6) is the form of the constraints we will use.

Before describing the results of the method, it is worth pointing out that the $\beta$ spiral result is not necessarily mass conserving. As Olbers et al. (1985) point out (their equation (30)) the mass conservation would require the nondivergence of the absolute velocity,

$$
u_{x}+v_{y}+w_{z}=0
$$

Although the relative velocity is identically nondivergent, the reference velocity obtained by equation (3-6) will not necessarily be so. We shall come back to this point later again in Section 4.2. 


\subsection{Formulation}

Figure 3-10 shows the number of data points within the European Basin (Figure 2-7) and within the $12^{\circ}$ square as a function of depth. The number of deep stations in this region decrease quickly beyond $4000 \mathrm{dbar}(29)$. In calculating the modes, values of the mean vertical profile were supplemented at depths deeper than the ocean floor (Section 3 of the previous chapter). This would mean that the mapped field at depth may be influenced by the shallower stations. The objective mapping step might in effect use the mean profile values at depth to cause some spurious structure at the deeper levels. With this consideration, depth 28 ( $3750 \mathrm{dbar})$ is a reasonably safe choice as the maximum depth that we analyze. Furthermore, to avoid possible boundary effects and errors caused by large scale smoothing of topographic features, the maximum depth that was included in the analysis was further reduced to 2 standard depths above the bottom of each station and its surroundings. The shallowest depth we use is $200 \mathrm{dbar}$ (standard depth 5), which is chosen to avoid the mixed layer where non-adiabatic effects due to air-sea interaction could be significant and also to avoid the shallow levels with large residuals (Section 5 of the previous chapter).

Potential density is used in place of density in equation (3-6). Between the shallowest level (200 dbar) and $1000 \mathrm{dbar}$ (standard depth 14), $\sigma_{0}$ is used, while $\sigma_{2}$ is used below 1000 dbar to 3000 dbar (standard depth 25). $\sigma_{4}$ is used below 3000 dbar.

At each grid point, we have the equation of the form (3-6) at each standard depth, typically from 200 dbar (standard depth, 5) to 3750 dbar (28), which amount to 24 equations for the 3 unknown reference velocities at that grid point. This formula defines a linear inverse problem.

The inversion requires the nondimensionalization of each equation by its respective accuracy. From the results of objective mapping, we have an error estimate of the modal expansion coefficients at each grid point. Estimates of the accuracy of the hydrographic 


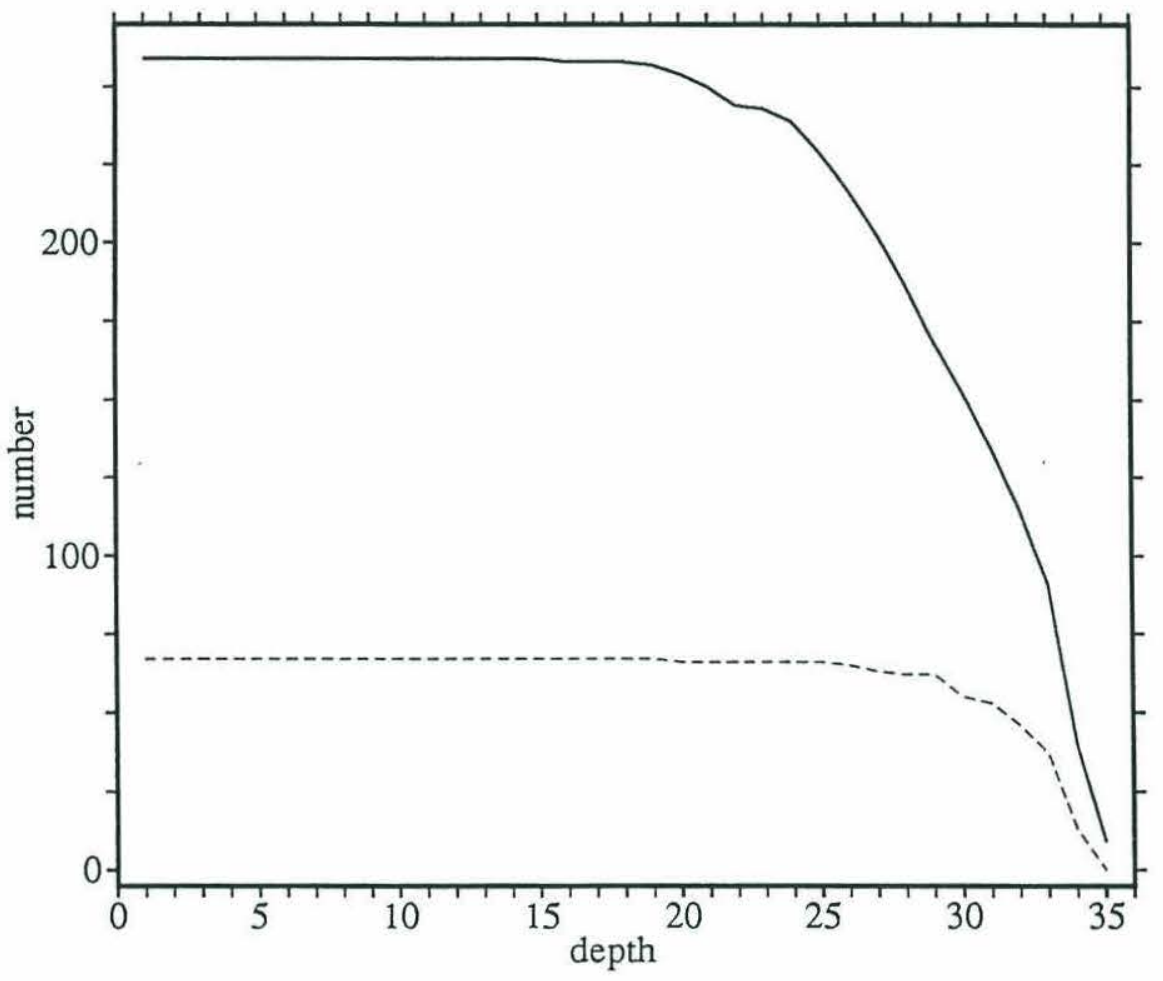

Figure 3-10. Number of Stations

Solid curve is within the European Basin, dashed curve is within the $12^{\circ}$ square. 
variables can be calculated from these expansion coefficient errors. Temperature, for example, is expanded in the modes as,

$$
T_{j}=T_{0 j}+\sum_{i=1}^{6} v_{i} U_{i j}
$$

$T_{j}$ is temperature at standard depth $j, T_{0 j}$ is the mean temperature at standard depth $j, U_{i j}$ is the element of mode $i$ at depth $j$ multiplied by the depth's normalization factor $\sigma_{j}$ (equation 1-6) and eigenvalue $\lambda_{i} . v_{i}$ is the expansion coefficient of mode $i$. Then, the error of mode $i$ 's contribution to temperature, $\delta T_{i j}$, is,

$$
\delta T_{i j}=\delta v_{i} U_{i j},
$$

where $\delta v_{i}$ is error of $v_{i}$. Since each modal contribution to temperature is independent of one another, the error of temperature at depth $\mathrm{j}, \delta T_{j}$, can be estimated by,

$$
\delta T_{j}=\sqrt{\sum_{i}^{6} \delta T_{i j}^{2}}=\sqrt{\sum_{i}^{6} \delta v_{i}^{2} U_{i j}^{2}} .
$$

Errors of other derived quantities are estimated similarly by the square root of the sum of squared modal contribution errors.

Assuming that the errors in the expansion coefficients for temperature and salinity are fully correlated, the error of potential density due to error of mode $i$ at depth $j$ is,

$$
\delta \sigma_{i j}=\frac{\partial \sigma}{\partial T} \delta T_{i j}+\frac{\partial \sigma}{\partial S} \delta S_{i j}
$$

The error contribution of mode $i$ to dynamic height at depth $j$ (referenced to 2000 dbar) can be estimated by,

$$
\delta h_{i j}=\int_{2000}^{p_{j}}\left(\frac{\partial}{\partial T}\left(\frac{1}{\rho}\right) \delta T_{i}+\frac{\partial}{\partial S}\left(\frac{1}{\rho}\right) \delta S_{i}\right) d p .
$$

Horizontal gradients, which appear in the $\beta$-spiral equations and the dynamic calculation for the geostrophic velocities, were estimated by central differences over $2^{\circ}$ 
latitude and longitude. The errors of variables involving differentiated quantities depend on the correlation over the $2^{\circ}$ width over which they are differentiated. Objective mapping provides an estimate of these covariances as well as the error estimate at each grid point. On the other hand, the error estimates of the expansion coefficients are fairly uniform in this $12^{\circ}$ region (Figure 3-2). Thus to make computation simple, we have used a uniform value for the error, $\delta v_{i}$, and assumed a correlation function of form, $\exp -(x / 1000 \mathrm{~km})^{2}$, which is a close analogue of the correlation function used in mapping.

From these error estimates, we can compute the expected error of the RHS of equation (3-6). Each modal contribution to the overall error is computed independently of the other modes. The average of this error over the $12^{\circ}$ region is shown in Figure 3-11 as a function of standard depth. The RHS error at standard depth 21 is identically zero, as this depth is the reference surface. On the other hand, the accuracy of each equation also depends on how accurate the left hand side can be evaluated by the $\beta-$ spiral method. The expected magnitude of the reference velocities in this region are on the order of several $\mathrm{mm} / \mathrm{s}$. As a conservative estimate, we may expect the errors of $u_{0}, v_{0}$ to be about $5 \times 10^{-4} \mathrm{~m} / \mathrm{s}$. The vertical velocity at $2000 \mathrm{dbar}$ is roughly a factor of $10^{-4}$ smaller than the horizontal velocities, so the expected error of $w_{0}$ is about 5 $\times 10^{-8} \mathrm{~m} / \mathrm{s}$. These errors will be compared later with the result of the analysis. From these parameter error estimates, we compute the error of the left hand side of equation (3-6). The average LHS error over the region is shown as the dashed line in Figure 3-11. This error dominates near the reference level while the RHS error is dominant at shallower and deeper depths. The sum of the RHS error and the LHS error gives the average error of equation (3-6). We shall use this sum as the normalization of each equation (i.e. row weight). 


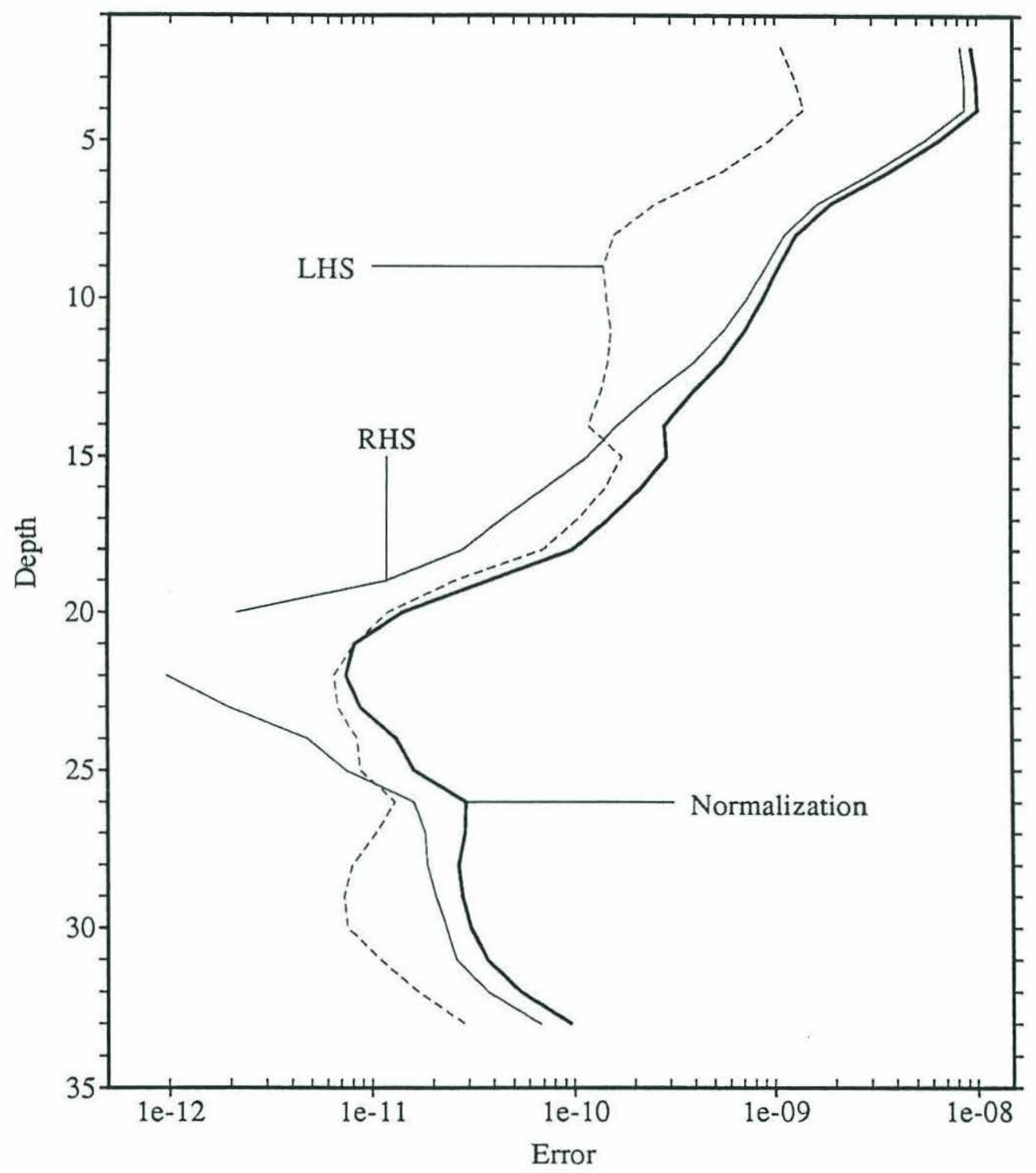

Figure 3-11. Error Estimate of $\beta$-Spiral Equations

Thin solid curve is error of the RHS, dashed curve is error of LHS, and thick solid is the total error of the $\beta$-spiral equations. 


\section{\$.९ Result of $\beta$-spiral Calculation}

The inversion is carried out at each degree latitude and longitude between $30^{\circ} \mathrm{W}$ to $20^{\circ} \mathrm{W}$ and $25^{\circ} \mathrm{N}$ to $35^{\circ} \mathrm{N}$ ( 121 grid points). The vertical profiles of the RHS's normalized by the row weights are shown in Figure 3-12. There are quite large values at middepths between 1000 dbar (14) and 1500 dbar (19) and also some close to the surface $(\sim 300$ dbar $(7))$. Places where the absolute value of this normalized RHS are larger than 1 indicate that the geostrophic flow is substantially at an angle to the density surfaces. In other words, the null solution, $u_{0}=v_{0}=w_{0}=0$, does not satisfy equation (3-6), and so equation (3-6) must be solved to determine the reference level velocities. The inversion is performed at each degree latitude and longitude using the singular value decomposition (e.g. Wunsch, 1978).

Many of the grid points have two dominant eigenvalues. At some places the inclusion of the third eigenvalue changes the solution drastically from that determined from the first two eigenvalues alone. The rank is chosen to be two uniformly. The residuals of these solutions are shown in Figure 3-13. Most of the large residuals about standard depth $16(1200 \mathrm{~m})$ have been reduced to a range indistinguishable from noise $(<1)$. So the inverted solutions do appear to satisfy the $\beta$-spiral equations.

The reference level velocities are shown in Figure 3-14. The magnitude $(\mathrm{mm} / \mathrm{sec})$ of the horizontal and vertical velocity components are about 1 and $10^{-4}$ at large areas, respectively. The mean error $(\mathrm{mm} / \mathrm{sec})$ of these components determined from the inversion are 0.1 and $10^{-5}$, respectively. Thus the major parts of the reference level circulation are larger than the noise and the coherent pattern we observe in Figure 3-14 appears to be significant. However, while the parameter resolutions for $v_{0}$ and $w_{0}$ have a mean value (over the 121 grid points) of 0.9 , that for $u_{0}$ is only 0.2 . Thus the east-west component is really not resolved by the $\beta$-spiral method and the values must be explored with caution. The magnitude of the error estimates are somewhat smaller than the a priori estimate used in the row weight calculation, but judging from the low 


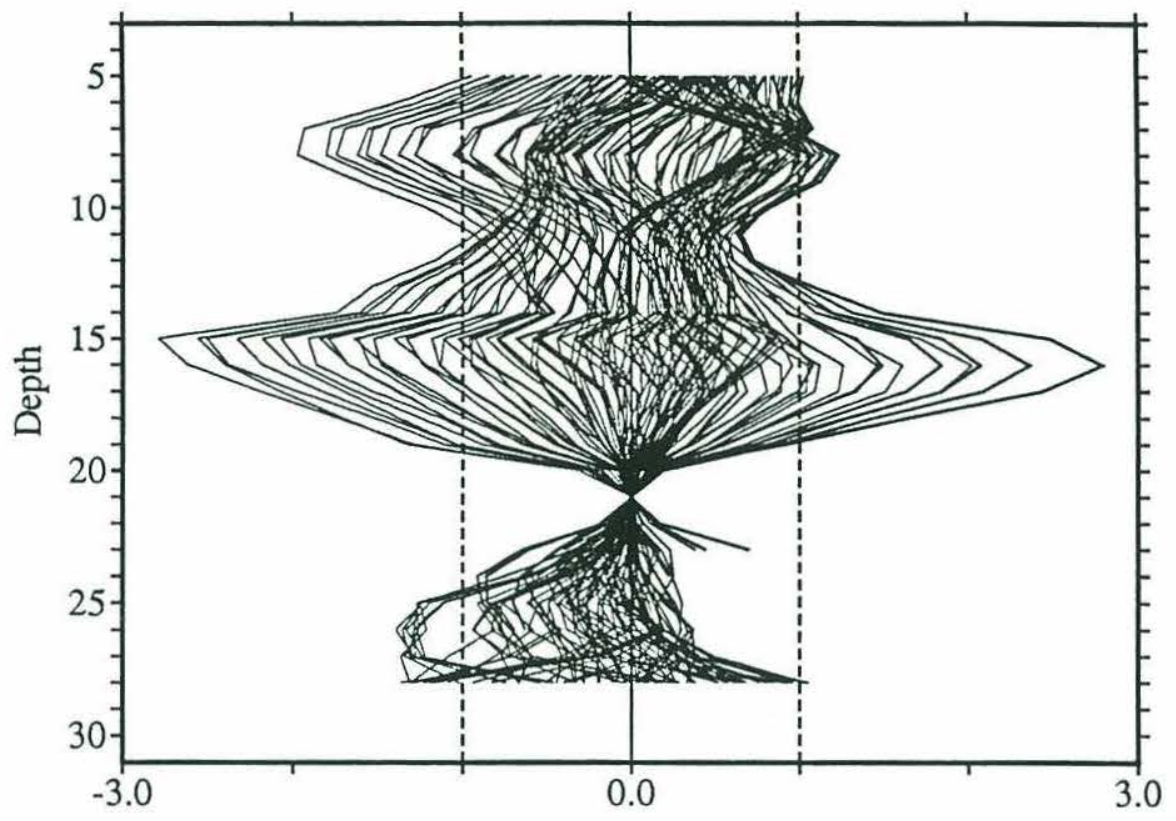

Figure 3-12. Normalized $\beta$-Spiral RHS

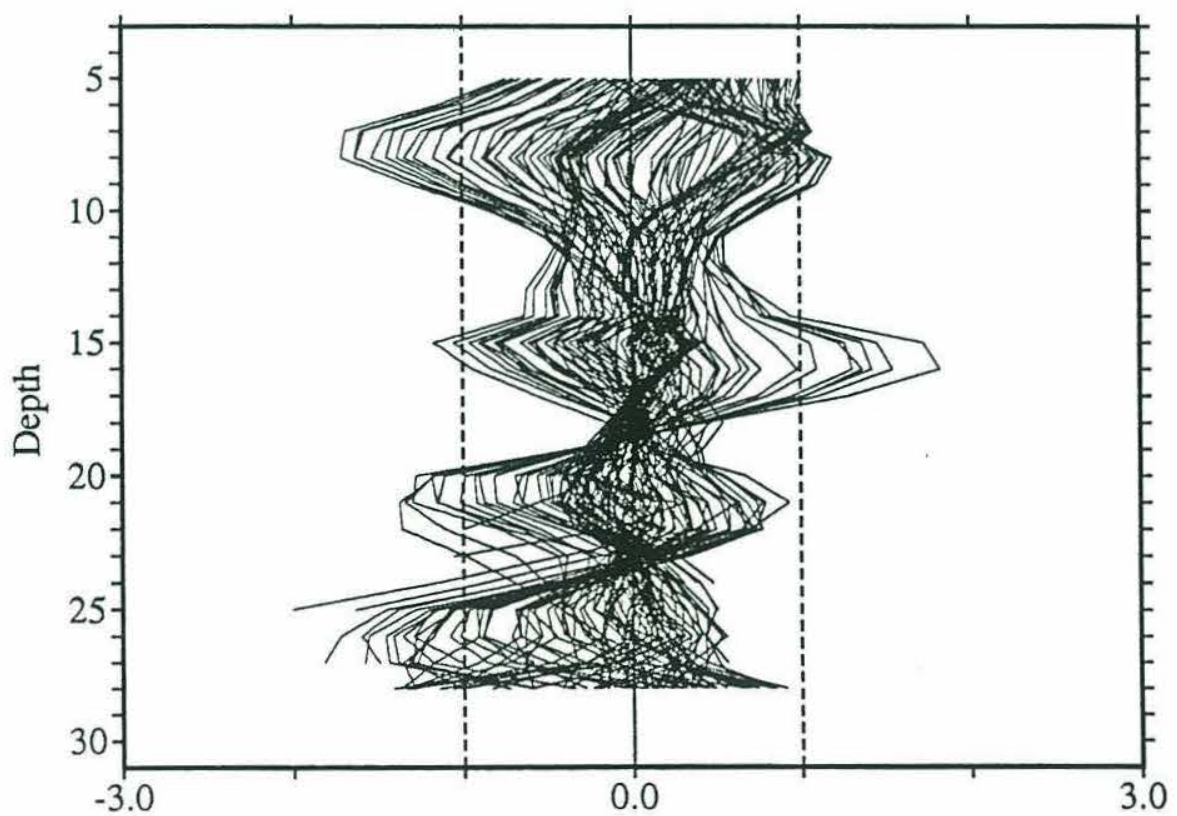

Figure 3-13. Normalized Residual of $\beta$-Spiral Equations (rank 2) 

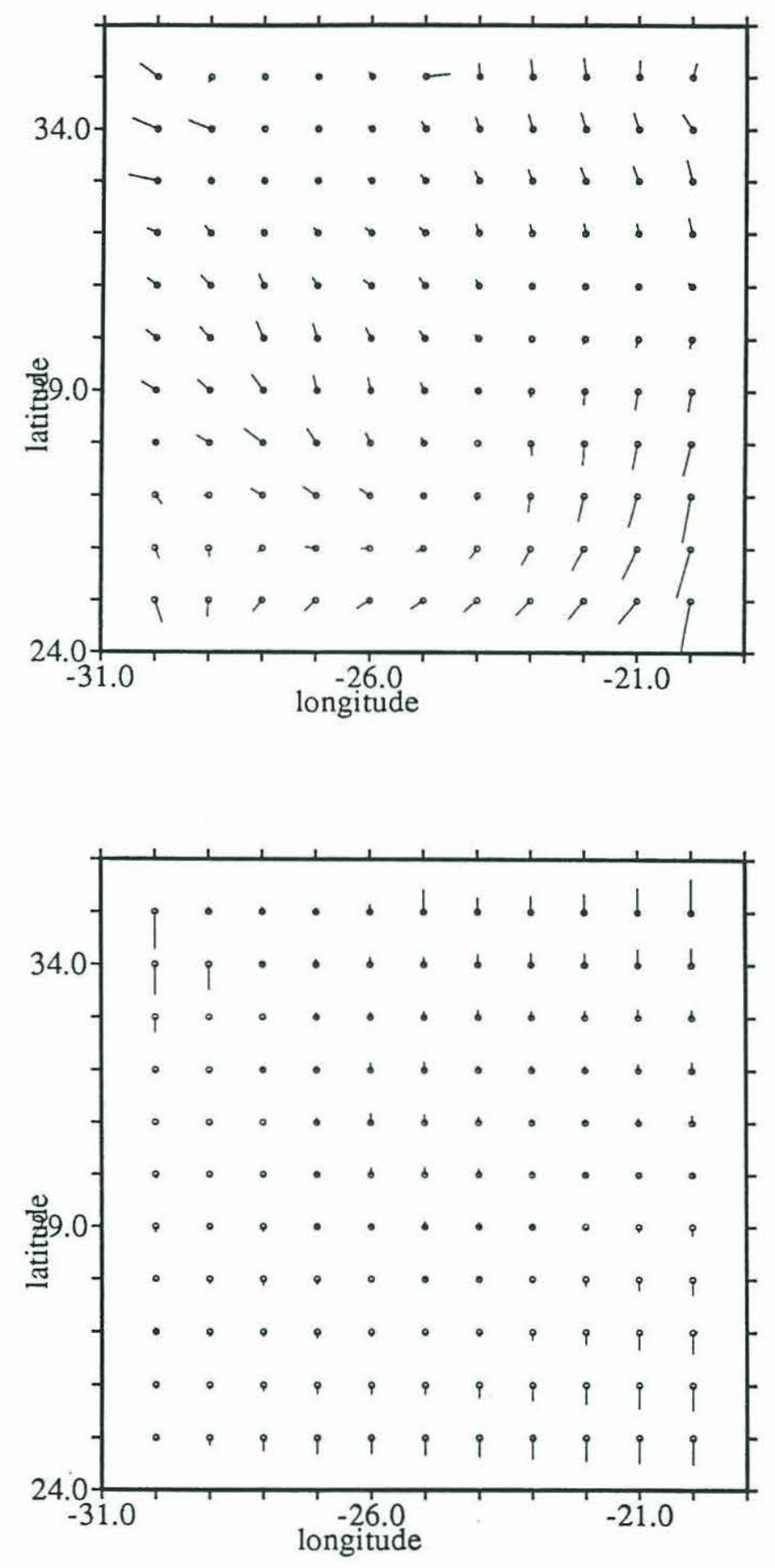

Figure 3-14. Reference Level Velocity Determined by $\beta$-Spiral (a) Horizontal component. $1^{\circ}=1(\mathrm{~mm} / \mathrm{sec})$

(b) Vertical component (positive northward). $1^{\circ}=10^{-4}(\mathrm{~mm} / \mathrm{sec})$ 
resolution of $u_{0}$, we can say that the $a$ priori weighting (Figure 3-11) is consistent with the solution.

The reference level circulation consists of a north-south divergent flow in the eastern region and a northwestward flow at the west. In the vertical, the flow is upwards in the north and downwards in the south. On the other hand, these reference level velocities are an order of magnitude smaller than the geostrophic velocities at shallower levels (order $1 \mathrm{~cm} / \mathrm{sec}$ ), so that absolute circulation at these depths ( 500 dbar) are almost the same as those shown in Figure 3-8.

\section{Box Model}

\subsection{Method and Formulation}

The box model is a regression method which employs kinematic and dynamic statements applied over a finite volume of water (box) to constrain the reference level velocities. It is essentially equivalent to integrating the $\beta$-spiral equations over a finite volume, using the divergence theorem. The basic constraints are conservation statements over a volume, such as mass. What goes in must come out, or balance the temporal change in the box. Wunsch (1978) gives a comprehensive treatment of the box model and the mathematics involved in solving it.

In this present problem, we divide the $12^{\circ}$ region into boxes of size $2^{\circ}$ latitude and longitude (Figure 3-15). Since the box model only constrains velocity components perpendicular to box boundary, we use a nested set of boxes as shown in Figure 3-15 to determine both east-west and north-south components at every $2^{\circ}$.

In the vertical, we define four layers bounded by five density surfaces (Table 3-1). In Chapter 2, we have found that there are only six primary degrees of freedom in the vertical, of which the third and fourth were important mostly in the tropics. In our model, we ignore the near surface layers and the abyssal waters, so four layers appears to be enough to resolve the circulation. The layer boundaries are spaced far 


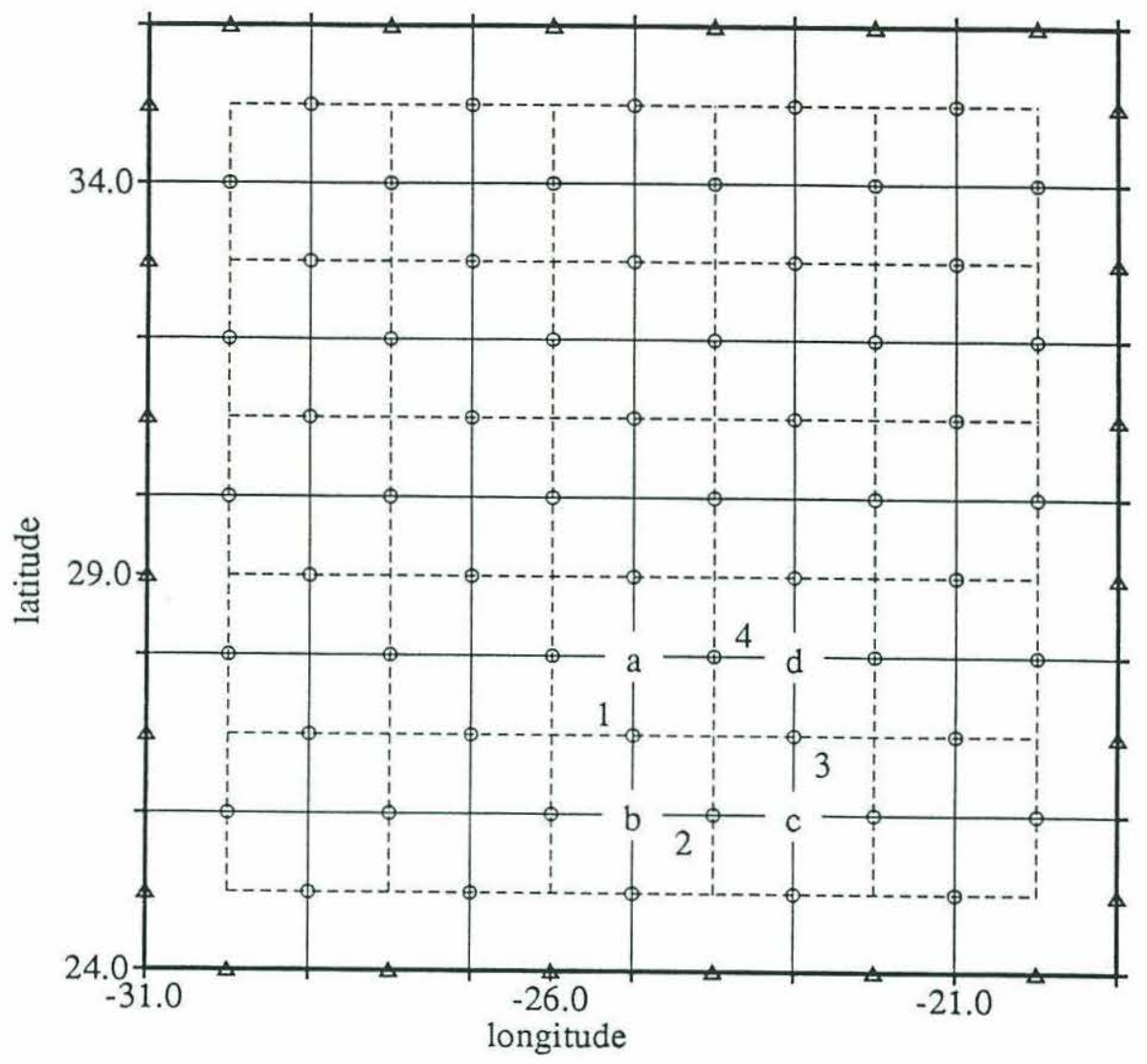

Figure 3-15. Box Model Geometry with Example Box abcd.

Reference level velocity are estimated on the circled grid points (e.g. 1, 2, 3, 4). Boxes ( $2^{\circ}$ on each side) bounded by solid lines each constrain one component of the reference velocity at the circles while the boxes with dashed lines constrain the other component. Only one component is estimated along the outer edge of the $12^{\circ}$ square. (At the triangle grid points.)

\begin{tabular}{|c|c|c|}
\hline depth & layer boundary & nominal depth (dbar) \\
\hline 1 & $26.8\left(\sigma_{0}\right)$ & 250 \\
\hline 2 & $27.5\left(\sigma_{0}\right)$ & 1000 \\
\hline 3 & $36.9\left(\sigma_{2}\right)$ & 1700 \\
\hline 4 & $37.0\left(\sigma_{2}\right)$ & 2550 \\
\hline 5 & $45.85\left(\sigma_{4}\right)$ & 3700 \\
\hline
\end{tabular}

Table 3-1. Layer Boundary of Box Model 
enough from each other so that they will not be redundant. (The resolution matrix, Section 6 of Chapter 2, indicated high correlation over about five standard depths.) The second layer (roughly the Mediterranean Water) is the depth range where the $\beta$-spiral equations showed significant residuals for the null solution.

Mass conservation will be used as the constraining equation. Assuming cross isopycnal flow to be insignificant, we can write the mass conservation, for example, in a box $a b c d$ in Figure $3-15$ as,

$$
u_{1} S_{a b}+v_{2} S_{b c}-u_{3} S_{c d}-v_{4} S_{d a}=0 .
$$

$u_{i}, v_{i}$ are eastward and northward velocities at point $i . S_{a b}$ is the area on the side of the box between points $a$ and $b$. Using equation (3-2), this can be written,

$$
u_{01} S_{a b}+v_{02} S_{b c}-u_{03} S_{c d}-v_{04} S_{d a}=-\left(u_{1}^{\prime} S_{a b}+v_{2}^{\prime} S_{b c}-u_{3}^{\prime} S_{c d}-v_{4}^{\prime} S_{d a}\right) . \quad(3-14)
$$

The right hand side is the mass flux divergence of the geostrophic velocity referenced to $2000 \mathrm{dbar}$. This equation provides a constraint for determining the unknown reference velocities, $u_{0}, v_{0}$ at grid points $(1,2,3,4)$. Layers vertically above and below provide similar constraints on the same set of velocities, and boxes adjacent to $a b c d$ constrain the velocity component shared by the two boxes. These equations applied to the series of boxes in Figure 3-15 define the linear inverse problem of the box model.

The accuracy of equation (3-14) is estimated in a similar manner to the $\beta$-spiral equations. The error of the LHS and RHS of equation (3-14) are shown in Figure 3-16 together with the sum of the two (the row weighting). The normalized RHS of equation (3-14) over the entire region are shown in Figure 3-17. There are very few significant values on the RHS and the inverse problem is essentially a homogeneous set of equations. This result implies that the geostrophic velocities with a level of no motion at $2000 \mathrm{dbar}$ are mass conserving. 


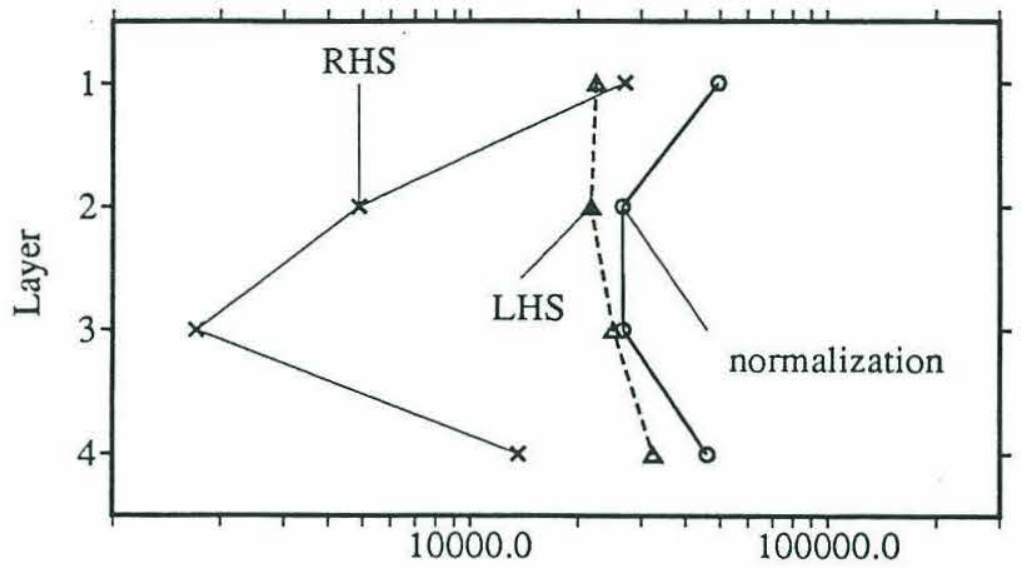

Figure 3-16. Weighting Factor for the Box Model

Crosses are RHS error, triangles the LHS error, and the circles are the total error (normalization factor for box model).

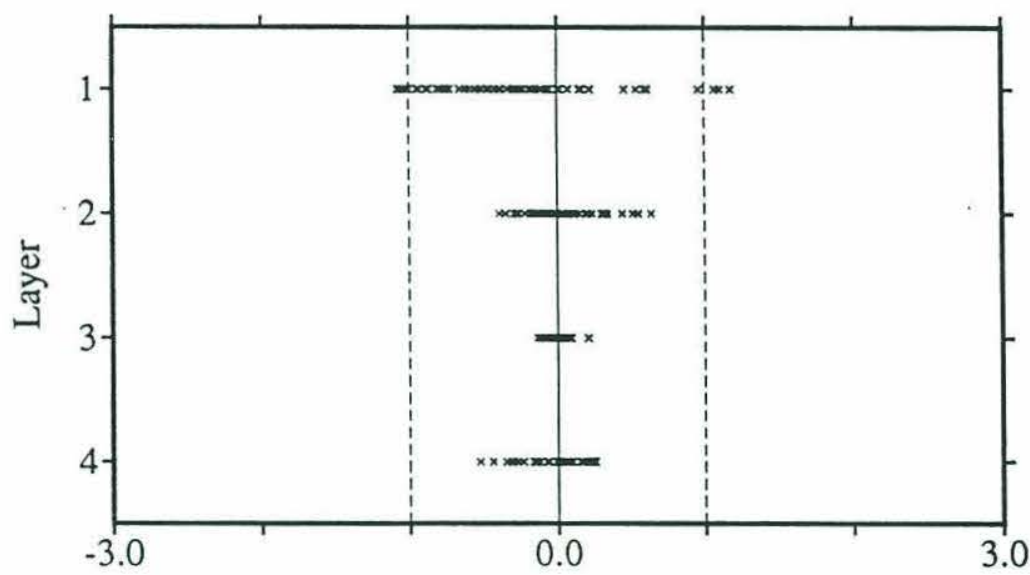

Figure 3-17. Weighted Box Model RHS

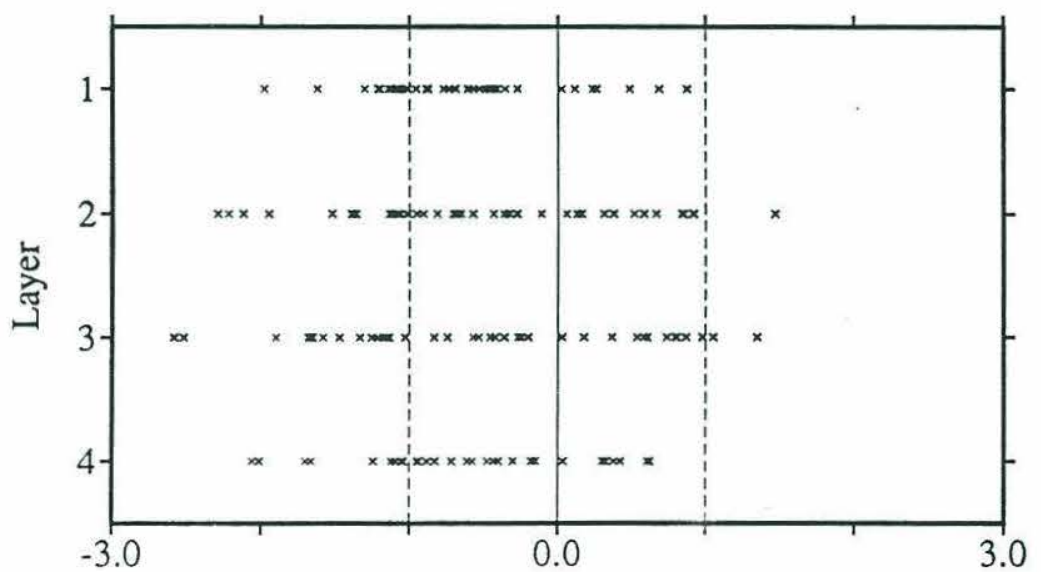

Figure 3-18. Mass Flux Divergence of $\beta$-Spiral Result 
This small mass flux divergence reflects the degeneracy of the box model over a small region. In a small region far away from boundary currents or rings which have strong density gradients, adjacent isopycnals are almost parallel (i.e. uniform thickness), and the Coriolis parameter constant. In such a situation, the total transport through a series of stations is dependent only on the dynamic height difference of the two end stations. In a closed box, where the starting point and end point are the same, the total flux through the boundary is identically zero. Let's show this in an example. For box abcd in Figure 3-15,

$$
\begin{aligned}
u_{1}^{\prime} & =-\frac{1}{f} \frac{D_{a}-D_{b}}{\Delta_{a b}}, \\
S_{a b} & =h \Delta_{a b},
\end{aligned}
$$

where, $D_{a}$ is dynamic height at point $a$ and $\Delta_{a b}$ is distance between points $a$ and $b$. Using these notations, the geostrophic flux through $a b$ is,

$$
u_{1}^{\prime} S_{a b}=-\frac{h}{f}\left(D_{a}-D_{b}\right),
$$

and the geostrophic mass flux divergence through all sides of box abcd is,

$$
u_{1}^{\prime} S_{a b}+v_{2}^{\prime} S_{b c}-u_{3}^{\prime} S_{c d}-v_{4}^{\prime} S_{d a}=-\frac{h}{f}\left\{\left(D_{a}-D_{b}\right)-\left(D_{c}-D_{b}\right)-\left(D_{d}-D_{c}\right)+\left(D_{d}-D_{a}\right)\right\}=0 .
$$

To summarize, the null solution, $u_{0}=v_{0}=0$ (level of no motion at $2000 \mathrm{dbar}$ ), satisfies mass conservation throughout the $12^{\circ}$ square, and no inversion is necessary to modify the initial reference level velocities.

\subsection{Is the $\beta$-spiral Result Mass Conserving?}

The box model shows the null solution to be consistent, while the $\beta$-spiral results in a nontrivial circulation. We have two sets of solutions and are left with the question of which one is the right one. What have we determined? We now examine this seemingly contradictory result. 
The $\beta$-spiral method estimates $u_{0}, v_{0}, w_{0}$ locally and the resulting velocity is not in general non-divergent. Mass conservation requires the velocity field to be both tangent to isopycnals ( $\beta$-spiral equations) and spatially non-divergent - a non-local requirement. Rewriting equations (3-5) and (3-7) in density coordinates (using the relations $\partial /\left.\partial x\right|_{z}=\partial /\left.\partial x\right|_{\sigma}-z_{x} / z_{\sigma} \cdot \partial /\left.\partial \sigma\right|_{\sigma}$, etc) we have, respectively,

$$
\begin{aligned}
& \left(u_{x}-u_{\sigma} \frac{z_{x}}{z_{\sigma}}\right)+\left(v_{y}-v_{\sigma} \frac{z_{y}}{z_{\sigma}}\right)+\frac{w_{\sigma}}{z_{\sigma}}=0 \\
& u z_{x}+v z_{y}-w=0 .
\end{aligned}
$$

By eliminating $w$ from equation (3-18) using equation (3-19) we have,

$$
\left(u z_{\sigma}\right)_{x}+\left(v z_{\sigma}\right)_{y}=0
$$

$z_{\sigma}$ is in essence thickness between isopycnals. Use of the divergence theorem to equation (3-20) yields mass conservation between isopycnals, which is the constraint used in the box model.

Figure 3-18 is the mass flux divergence, equation (3-13), when we use the result of the $\beta$-spiral (Figure 3-14). Equivalently, this is the residual of equation (3-14) when we use $\beta$-spiral results as $u_{0}, v_{0}$. The residuals are significantly larger than the error estimates. Compare this with the original RHS, Figure 3-17, when we use the null solution. Thus we conclude the result of the $\beta$-spiral method, Figure $3-14$, is not mass conserving.

We have now found that neither $\beta$-spiral method's solution nor the box model's solution is an overall consistent solution. The $\beta$-spiral result is not mass conserving and the box model solution does not satisfy the $\beta$-spiral equations. In the next section, we find an overall consistent solution. 


\section{Hybrid Model}

\subsection{Formulation}

In the previous sections, we have found that neither the $\beta$-spiral alone or the box model alone can determine an overall consistent solution. The reason simply put is that neither model provides an optimum solution to the other if solved separately. So in this section, we shall examine a hybrid of the two methods. As they are both linear inverse problems, we can simply adjoin the two problems into the form,

$$
A \underline{b}=-\underline{\Gamma},
$$

where, now the upper part of the rows of the matrix equation is the box model and the lower part is the $\beta$-spiral constraints. To keep the matrix equation from being unnecessarily large, we have restricted the $\beta$-spiral equation to a subset of the standard depths used in Section 3. The subset consists of four standard depths: $8,13,16$, and 25 (400, 900, 1200, 3000 dbar). These are the depths where the original $\beta$-spiral RHS's have significantly large values (Figure 3-12). The boxes are the same as those used in Section 4 (i.e. 4 layers in the vertical, $2^{\circ}$ on each side). There are 204 unknown parameters to be solved. The reference velocities, $u_{0}, v_{0}, w_{0}$, are to be determined on 60 grid points inside the $12^{\circ}$ region. In addition, there are 12 grid points along $31^{\circ} \mathrm{W}$ and $19^{\circ} \mathrm{W}$ where only $u_{0}$ is solved for, and another 12 points along $24^{\circ} \mathrm{N}$ and $36^{\circ} \mathrm{N}$ where only $v_{0}$ is found. The number of equations for this hybrid problem is 451 . Of these, there are 224 equations for the box model (61 boxes for the shallowest layer) and 227 equations for the $\beta$-spiral (applied on the 60 grid points inside the $12^{\circ}$ region). The weighted RHS of equation (3-21) is shown in Figure 3-19. The RHS of the box model is the same as in Figure $3-17$. The equation that is not homogenous is the $\beta$-spiral equations. 

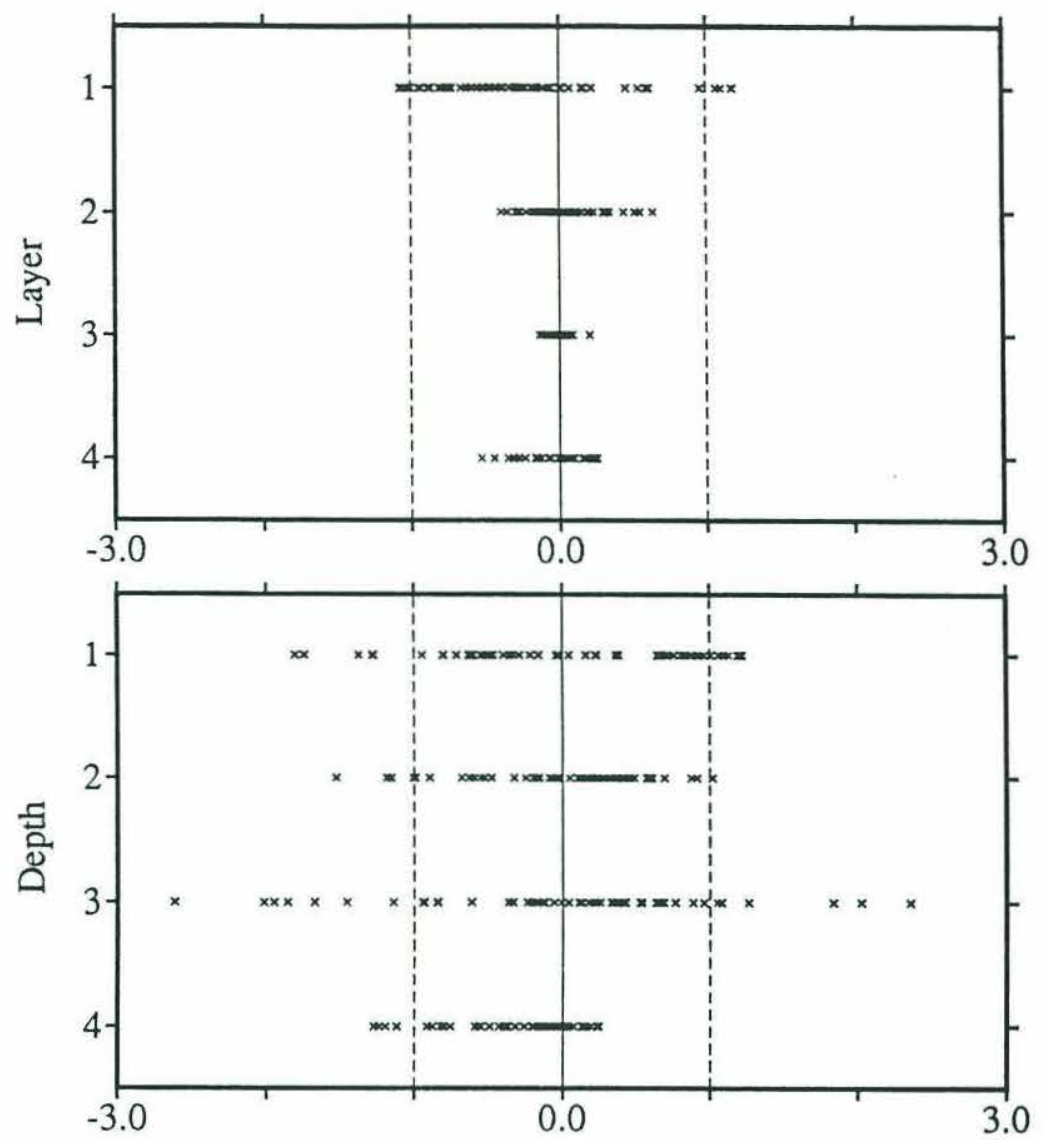

Figure 3-19. Normalized RHS of Hybrid Model

The top figure is for the box model equations, and the lower one is for the $\beta$-spiral equations.

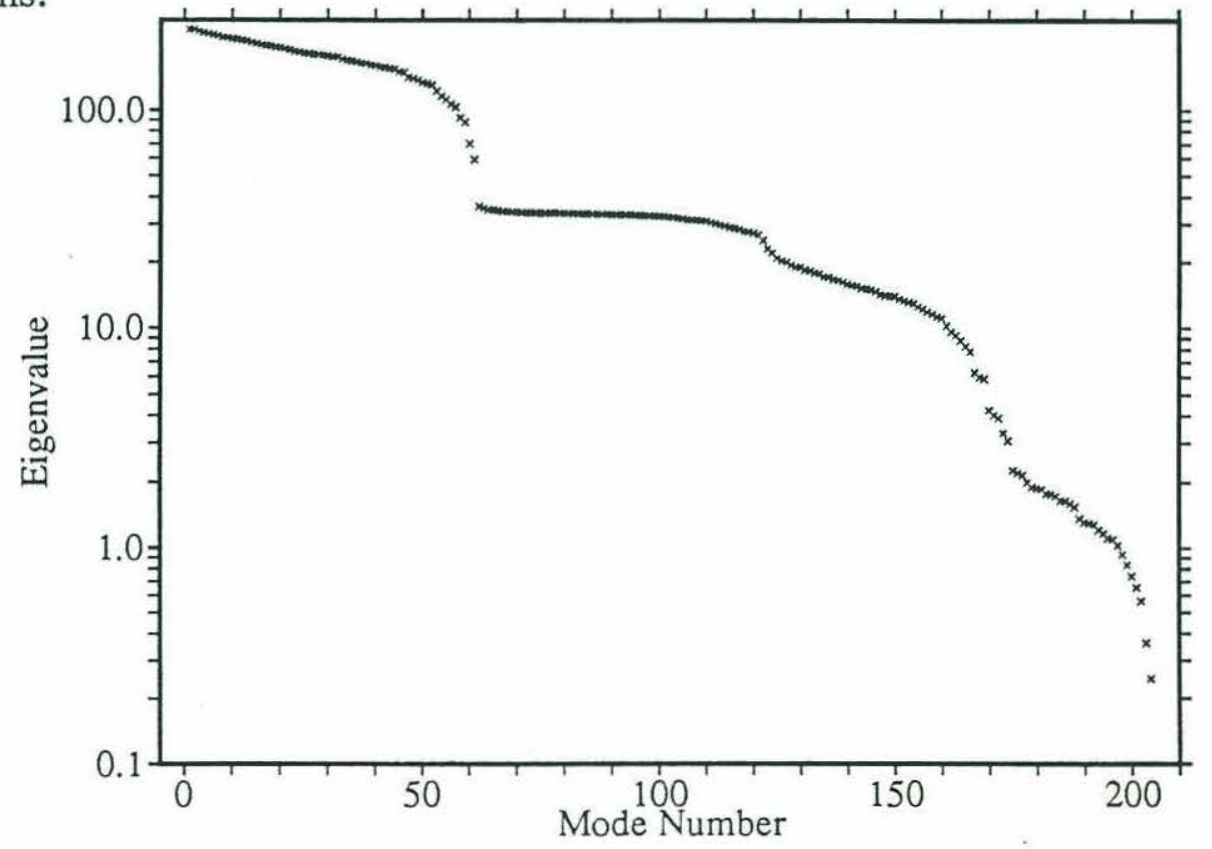

Figure 3-20. Eigenvalues of Hybrid Model 


\subsection{Result}

The inverse problem, equation (3-21), was solved by the singular value decomposition. The eigenvalues of matrix $A$ are shown in Figure 3-20. There is a jump in magnitude after the 60th eigenvalue, but they remain constant thereafter until about the 166th value, after which they quickly decrease. Figure 3-21 shows the so-called LevenbergMarquardt diagram (Lawson and Hanson, 1974), where we compare the solution norm (in weighted form) to the residual norm (also in normalized form) as we include more eigenvalues in the solution. The residual hardly changes at the beginning until about 70. Between 70 and 172 , the residual quickly decreases with little increase in the solution norm. Beyond 172, the decrease in residual is associated with large increase of solution norm, indicative of the small eigenvalues introducing spurious structure in the solution, due to errors in data.

Analysis of the diagonal elements of the equation resolution matrix shows that between eigenmodes 1 to 100, the second and third layers of the box models are primarily being resolved and explains why the residuals are not decreasing much at these eigenvalues. The $\beta$-spiral equations which have the large RHS are not used yet. On the other hand, it is primarily the $\beta$-spiral equations that become resolved beyond eigenvalue 100. From these results, we determine the rank of the problem to be 166 , which is at the corner of the Levenberg-Marquardt diagram.

The residuals of the equations are shown in Figure 3-22. Compare this with the original RHS (Figure 3-19). Almost all of the residuals of the $\beta$-spiral equations have decreased to acceptable noise values. Unlike the $\beta$-spiral method alone, the residuals of the box models have remained almost unchanged and mass is conserved. However, we note that the shallowest levels in the $\beta$-spiral equations still have a residual larger than the a priori estimate of error. This is similar to the result of the $\beta$-spiral method, where these shallow depths remain almost unchanged (Figures 3-12 and 3-13). In retrospect, the RHS and residual to the box model also have a somewhat larger value 


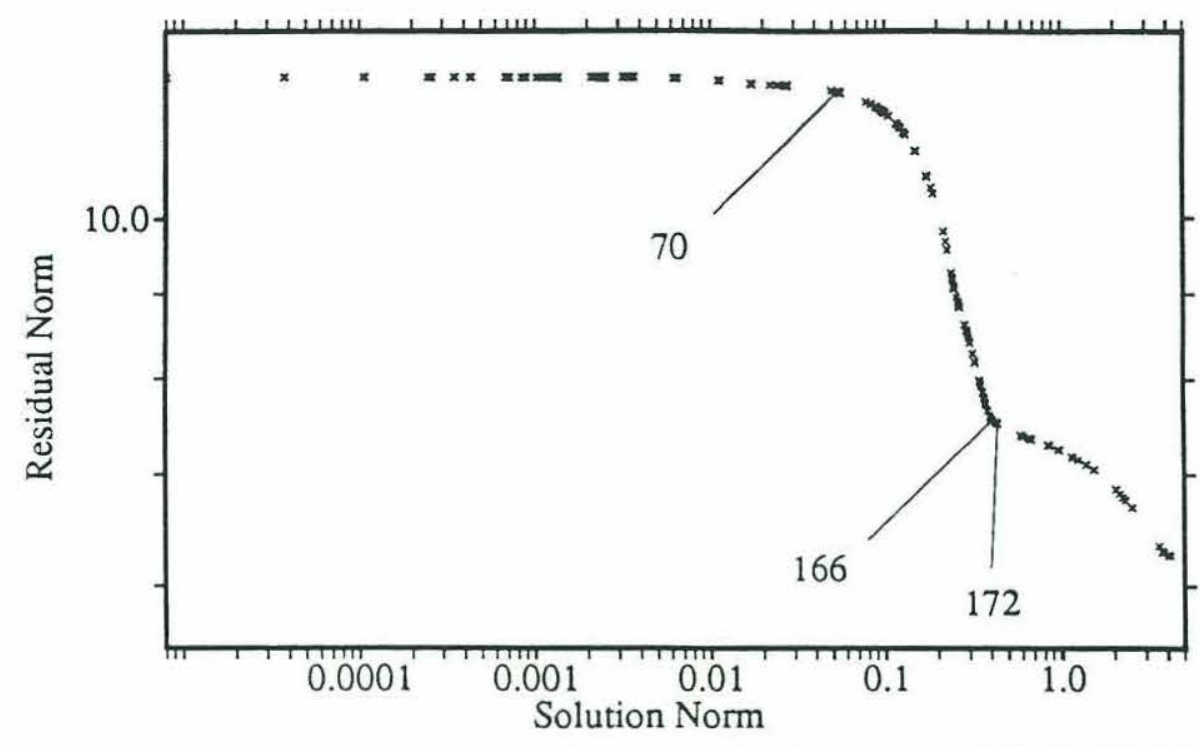

Figure 3-21. Levenberg-Marquardt Diagram of Hybrid Model

Labels on the plot are the mode numbers of the singular value decomposition.
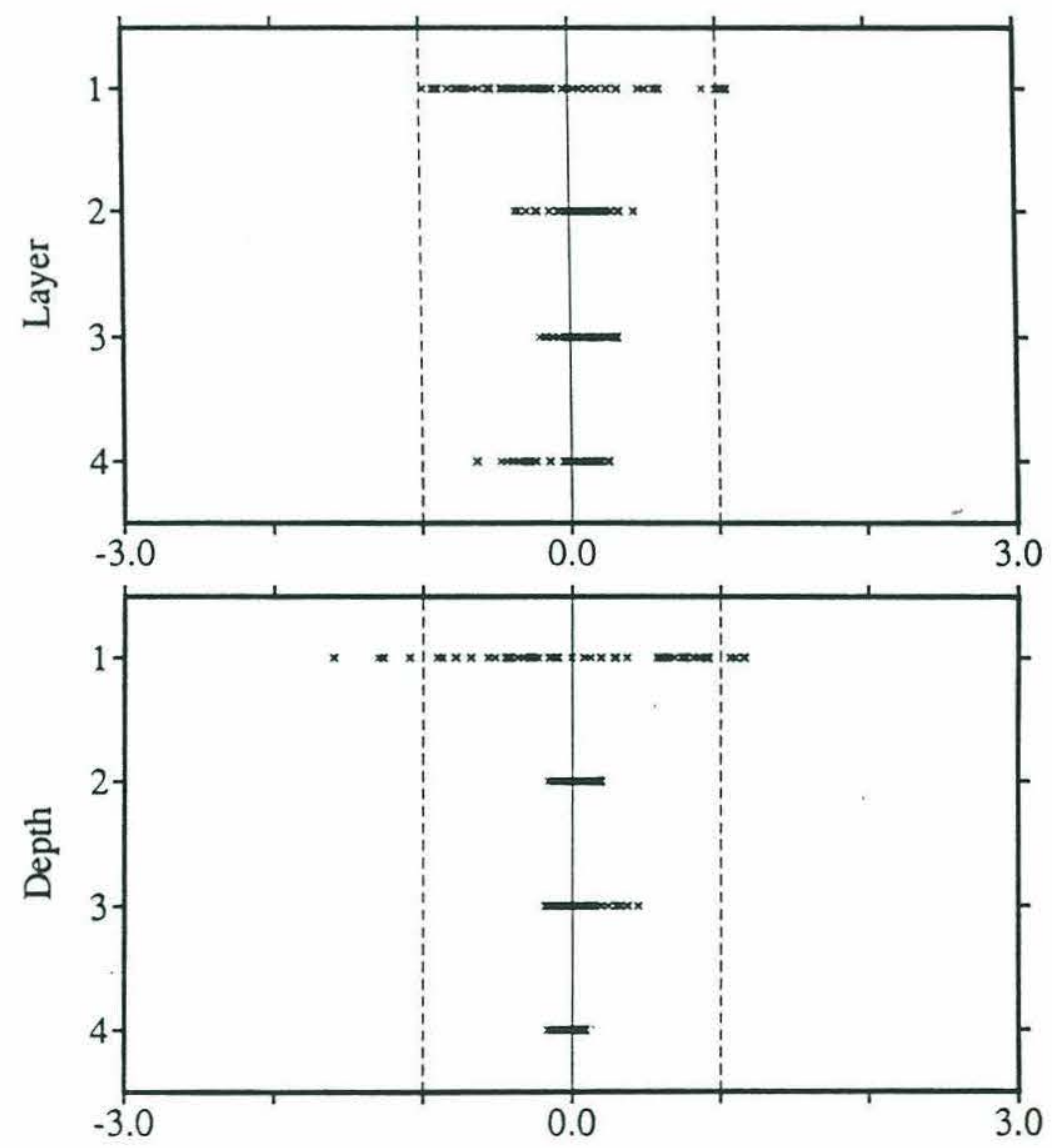

Figure 3-22. Normalized Residual of Hybrid Model

The top figure is for the box model equations, and the lower one is for the $\beta$-spiral equations, as in Figure 3-19. 
at the shallowest layer. This might be an indication that the model does not result in a consistent solution at these shallow depths. Perhaps mixing or time dependence are important.

The reference level velocities are shown in Figure 3-23. The horizontal components of the reference velocities are about $1 \mathrm{~mm} / \mathrm{sec}$ at stronger parts and the vertical velocities are on the order of $10^{-4} \mathrm{~mm} / \mathrm{sec}$. The determined reference level circulation has qualitatively similar features as the $\beta$-spiral result (Figure 3-14). The north-south divergent flow in the eastern half of the region and the north-south asymmetry in the upwelling pattern are apparent again. However, the westward flow in the western half in Figure 3-14 is now absent.

The parameter resolutions of $u_{0}, v_{o}$ and $w_{0}$ are all fairly uniform over the region and have mean values of $0.67,0.81$, and 0.99 , respectively. Compared with the $\beta$-spiral results, the east-west component is much better resolved.

However, the model parameter errors from this model are relatively large. The mean error $(\mathrm{mm} / \mathrm{sec})$ for $u_{0}, v_{o}$ and $w_{0}$ are $0.6,0.7$, and $2 \times 10^{-4}$, respectively. So in many parts of the model area the errors are almost as big as the estimates themselves, rendering the solution indistinguishable from noise. Thus it appears that the data may be too noisy to allow a significant solution to be determined by the model. On the other hand, the residuals of the shallowest level of the $\beta$-spiral and shallowest layer of the box model have excessive residuals compared to the rest. It may be that mixing or time dependence is essential at these shallow depths, and if so, we are introducing an inconsistency in the model by including the shallowest depths.

To take into account the possibility that there may be additional physics at the shallowest level, we have redone the calculation, excluding standard depth 8 of the $\beta$-spiral equation and the first layer in the box model. The eigenvalues, the relation between the sizes of solution norm and residual norm are all quite similar to the full model we had, and the rank is again chosen to be 166 . The estimated model parameters 

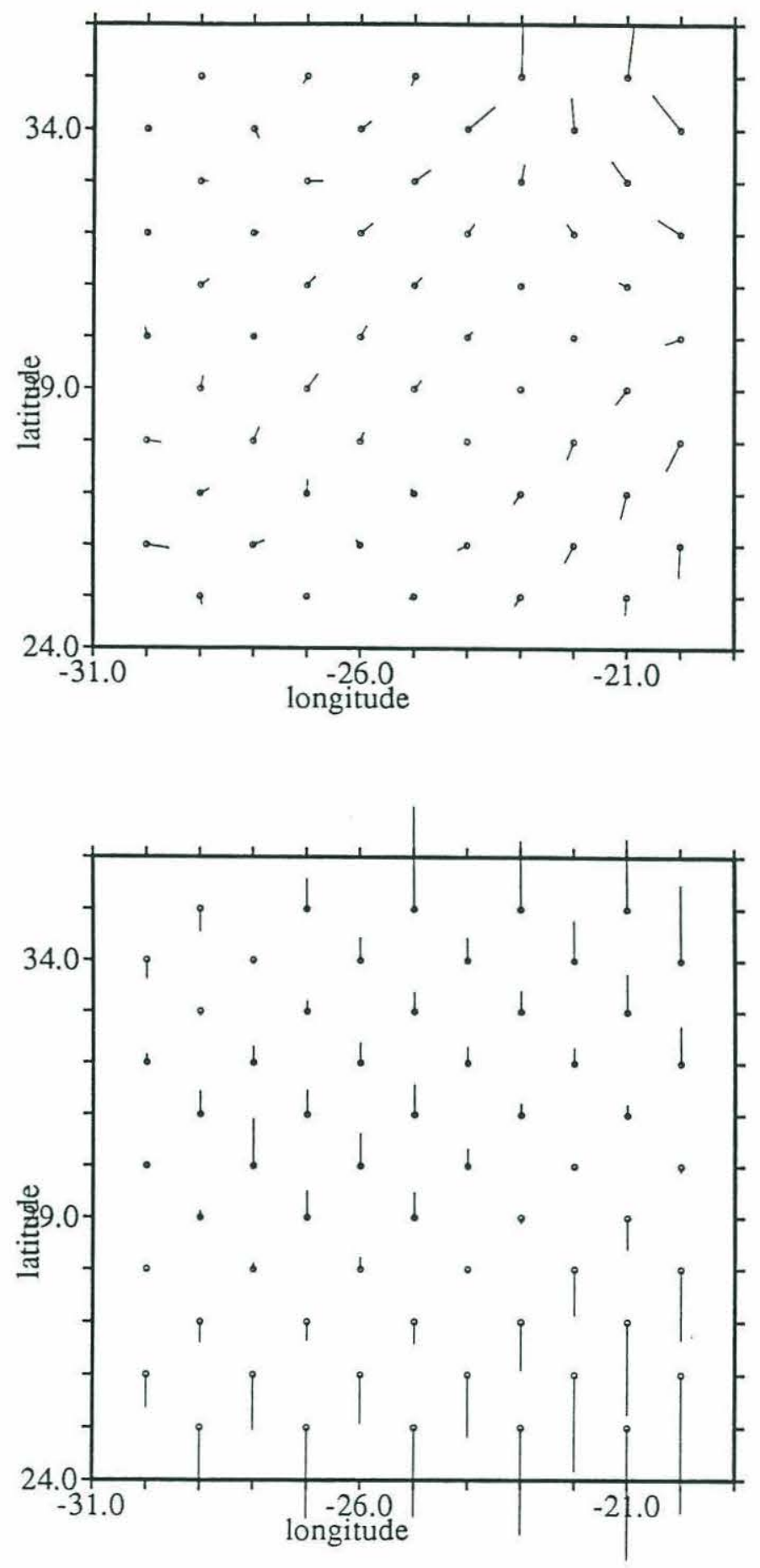

Figure 3-23. Reference Level Velocity of Hybrid Model

(a) Horizontal component $1^{\circ}=1(\mathrm{~mm} / \mathrm{sec})$

(b) Vertical component $1^{\circ}=10^{-4}(\mathrm{~mm} / \mathrm{sec})$ 


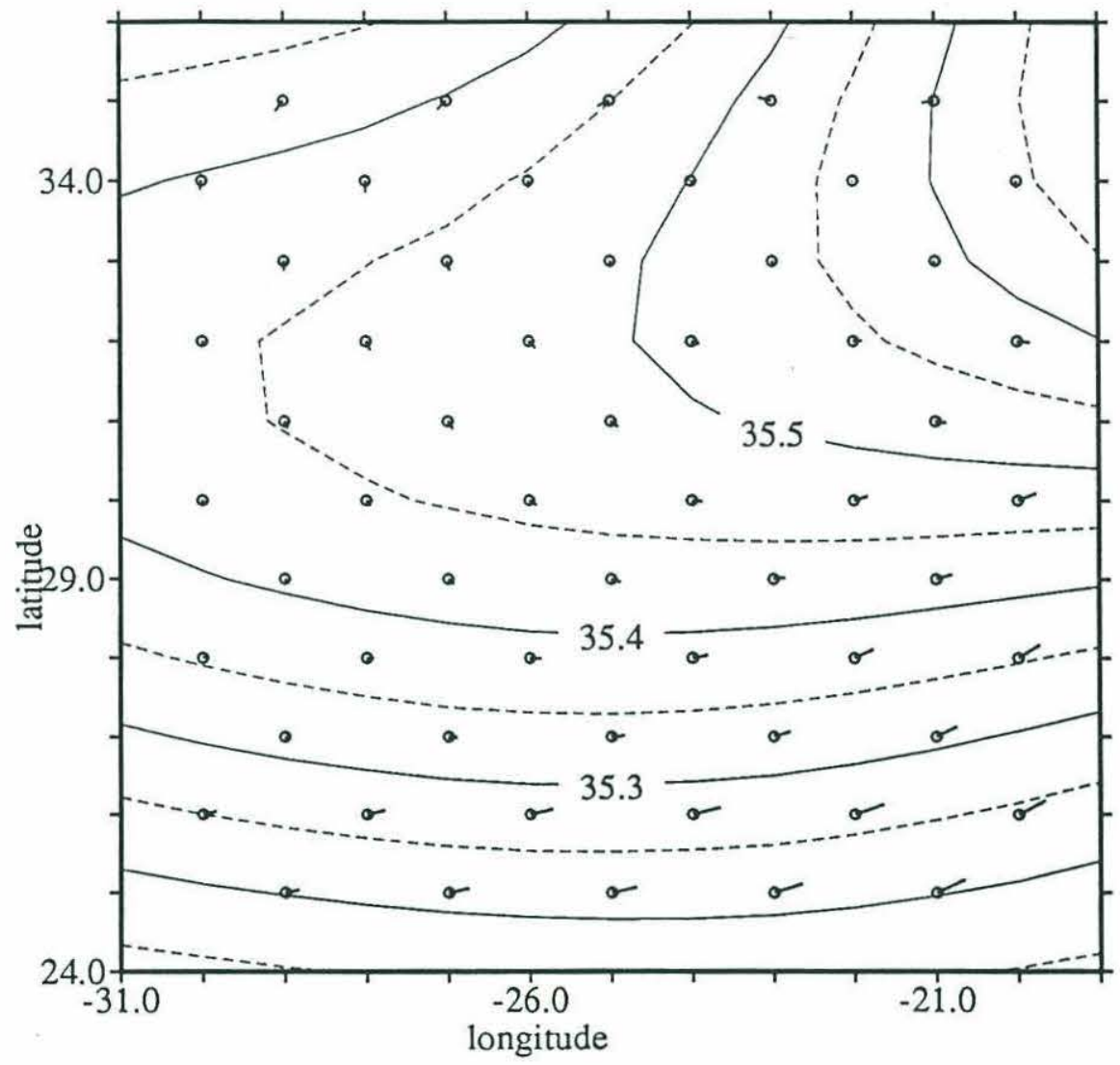

Figure 3-24. Absolute Flow at 1200 dbar. $1^{\circ}=1(\mathrm{~cm} / \mathrm{sec})$ 
are almost identical to those in Figure 3-23, as are the parameter resolutions. On the other hand, the error estimate $(\mathrm{mm} / \mathrm{sec})$ is now much smaller, with values of 0.2 for $u_{0}$ and $v_{0}$, and $6 \times 10^{-5}$ for $w_{0}$. With these error estimates, the major patterns we have identified in Figure 3-23 are larger than the noise and can be deemed significant.

Yet, the magnitude of these reference velocities are so small that, to the eye, the circulation at shallower depths would show little change. The absolute circulation (geostrophic plus reference level velocity) at $1200 \mathrm{dbar}$ is shown in Figure $3-24$. The effect of the reference velocities' (Figure 3-23) north-south divergence is to orient the northeastward flow at the southeast corner to become more eastward and the flow at the northeast to be westwards. (Compare Figures $3-24$ and $3-8 b$ ). The result is still a cyclonic circulation about the salinity anomaly of the Mediterranean tongue with little flow along its axis. This flow may seem inconsistent with the often anticipated westward spread of the Mediterranean Water. In the following section this result will be compared with previous results.

\section{Comparison to Other Observations}

Worthington (1976) argued that the subtropical gyre must be confined west of the Mediterranean salt tongue. Otherwise the presence of the Mediterranean tongue cannot be consistently explained by the southward return flow of the anticyclonic subtropical gyre. This view contrasts with the traditional descriptions of the basin wide wind driven circulation (e.g. Sverdrup et al., 1942).

Reid (1978) reanalyzed North Atlantic hydrographic data and found the middepth circulation consistent with salinity and density distributions. In contrast to Worthington's picture, the surface circulation above the Mediterranean tongue was dominated by the southward flowing subtropical gyre. On the other hand, the dynamic topography at $1000 \mathrm{~m}$ relative to $2000 \mathrm{~m}$ has a ridge with a characteristic "C" shape enclosing the high salinity anomaly of the Mediterranean tongue (Figure 3-25). This 


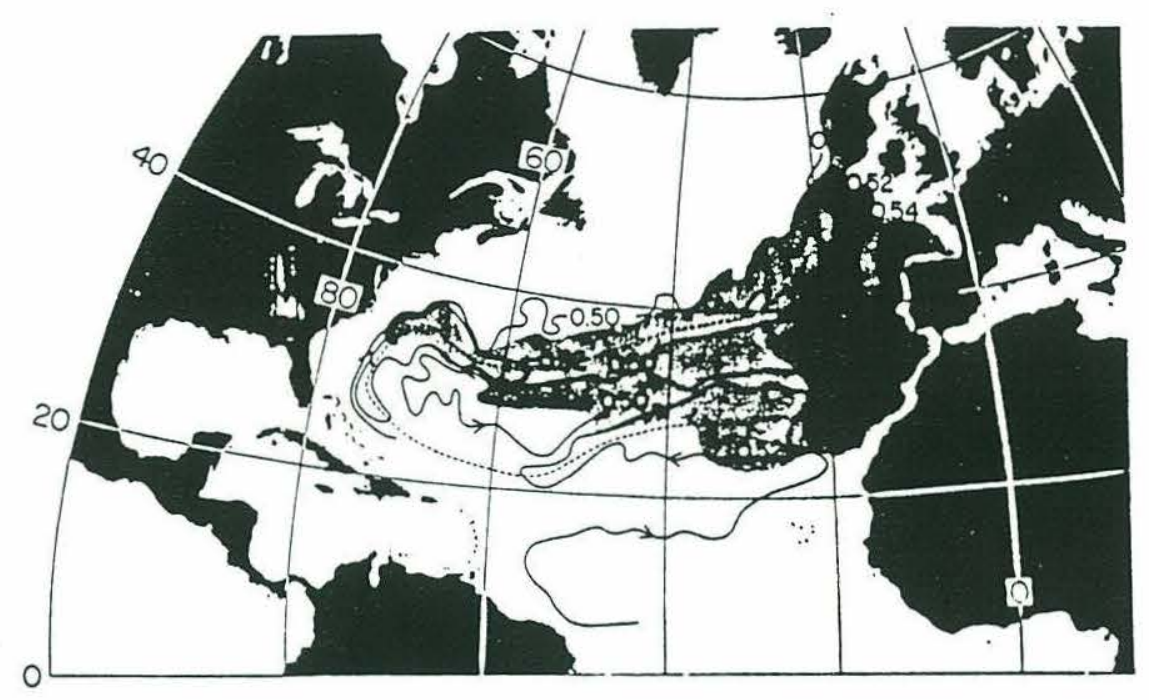

Figure 3-25. 1000 dbar Dynamic Topography relative to 2000 dbar Reprinted from Reid (1978, Fig. 4). The gray area represents salinity greater than 35.1 PSU at 1000 dbar. The dashed curve lies along the steric high, illustrating the $\mathrm{C}$ shape of the shear pattern.

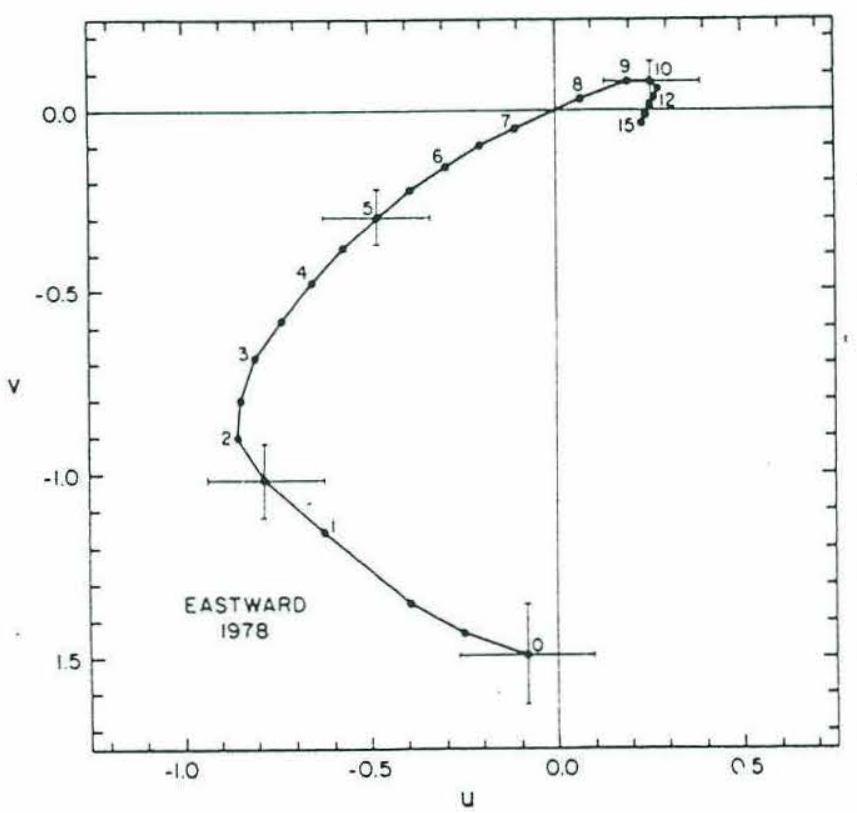

Figure 3-26. Absolute Velocity Hodograph at $27^{\circ} \mathrm{N}, 32.5^{\circ} \mathrm{W}$

Reprinted from Behringer and Stommel (1980, Fig. 5). Units are in (cm/sec). Numbers along the hodograph are depths in hundreds of meters. 
"C" shape extends across the entire basin. The circulation is to the southwest at the northern edge of the Mediterranean anomaly and east or northeast at the southern edge. The result of our inversion is consistent with Reid's picture of the circulation. There appears to be hardly any flow along the axis of the salt tongue; the flow is instead cyclonic around it.

Behringer and Stommel (1980) used the $\beta$-spiral method to determine the absolute flow using a synoptic hydrographic observation (rather than historical data, as earlier $\beta$-spiral applications did), in a triangular region just west of our $12^{\circ}$ square. This " $\beta$ triangle" (Armi and Stommel, 1983) is centered at $27^{\circ} \mathrm{N}, 32.5^{\circ} \mathrm{W}$ with $10^{\circ}$ on each side. Their absolute velocity hodograph is reproduced as Figure $3-26$. The flow above $750 \mathrm{~m}$ is to the south and west turning clockwise with depth, characteristic of the westward return flow of the subtropical gyre (Figure 3-9). The flow beneath $750 \mathrm{~m}$ is to the east and north with magnitudes of about $2 \mathrm{~mm} / \mathrm{s}$ at $1200 \mathrm{~m}$. This again is quite similar to our result.

Olbers et al. (1985) have applied the $\beta$-spiral method to determine the absolute circulation over the entire North Atlantic Ocean using Levitus' (1982) data. At the reference level $(2000 \mathrm{~m})$, their absolute flow is weak and not discernible from 0 in most parts of our $12^{\circ}$ region except for an eastward flow towards the south west corner of the region (several $\mathrm{mm} / \mathrm{sec}$ ). This flow appears variable and terminates before the eastern end of our $12^{\circ}$ region. This apparent horizontal divergence is due to the nature of the $\beta$-spiral method which does not guarantee nondivergence of velocity. There is a southward flow just on the east side of the region with comparable magnitude with what we have found, but there is no divergence to the north. These reference velocities like ours are so small that to the eye the absolute flow at shallower levels are almost identical with the geostrophic velocities. At $500 \mathrm{~m}$ the flow is dominated by the subtropical gyre. In the $12^{\circ}$ region, the westward return flow of the gyre is on 
the order of $1 \mathrm{~cm} / \mathrm{s}$, similar to our modal data. Their eastward flow turns westward at about $30^{\circ} \mathrm{N}$ similar to our result (Figure $3-8 \mathrm{a}$ ).

Hogg (1987) performed an analysis in a $24^{\circ}$ square $\left(19.5^{\circ} \mathrm{W}\right.$ to $43.5^{\circ} \mathrm{W}$ and $22.5^{\circ} \mathrm{N}$ to $46.5^{\circ} \mathrm{N}$ ) also using Levitus' data set. Using two density surfaces, one in the thermocline $(\sim 750 \mathrm{~m})$ the other in the core of the Mediterranean tongue $(\sim 1200 \mathrm{~m})$, Hogg fit an advection diffusion equation to the distributions of temperature, oxygen, and potential vorticity, while using a streamfunction to satisfy mass conservation. Hogg's circulation in the thermocline is dominated by the anticyclonic circulation of the subtropical gyre. The deeper level flow, on the other hand, consists of a westward flow which intensifies to the west. The difference at the deeper level between this result and ours (Figure 3-24) may be due to the difference in the data we use, which involve different data sets averaged differently. The difference may also be in the models we use. Hogg only uses two isopycnals, so the flow field may not be consistent with deeper flow. More significant may be the accuracies of the east-west velocity estimate. In our model, we found east-west velocity, $u_{0}$, to be relatively unresolved in comparison to the other two velocity components, and thus having more uncertainty. On the other hand, Hogg uses a streamfunction instead of velocity components, and so forces the solution to satisfy mass conservation exactly. This is equivalent to putting excessively large weight on the mass conservation equation relative to others, which may not be consistent with the errors in Levitus' data. The errors in the horizontal gradient of the streamfunction may not be the same for east-west derivative and north-south derivative.

When comparing results from separate studies, there is always the possibility that the differences in the results are real, and due to temporal changes. For example, Zenk and Müller (1988) find that a seven year current meter record at $1000 \mathrm{~m}$ depth near $33^{\circ} \mathrm{N} 22^{\circ} \mathrm{W}$ is not long enough to yield a statistically significant mean flow. Their vector time series, reproduced as Figure 3-27, displays strong interannual variability. Lagrangian velocity measured by SOFAR floats display a near zonal low frequency 


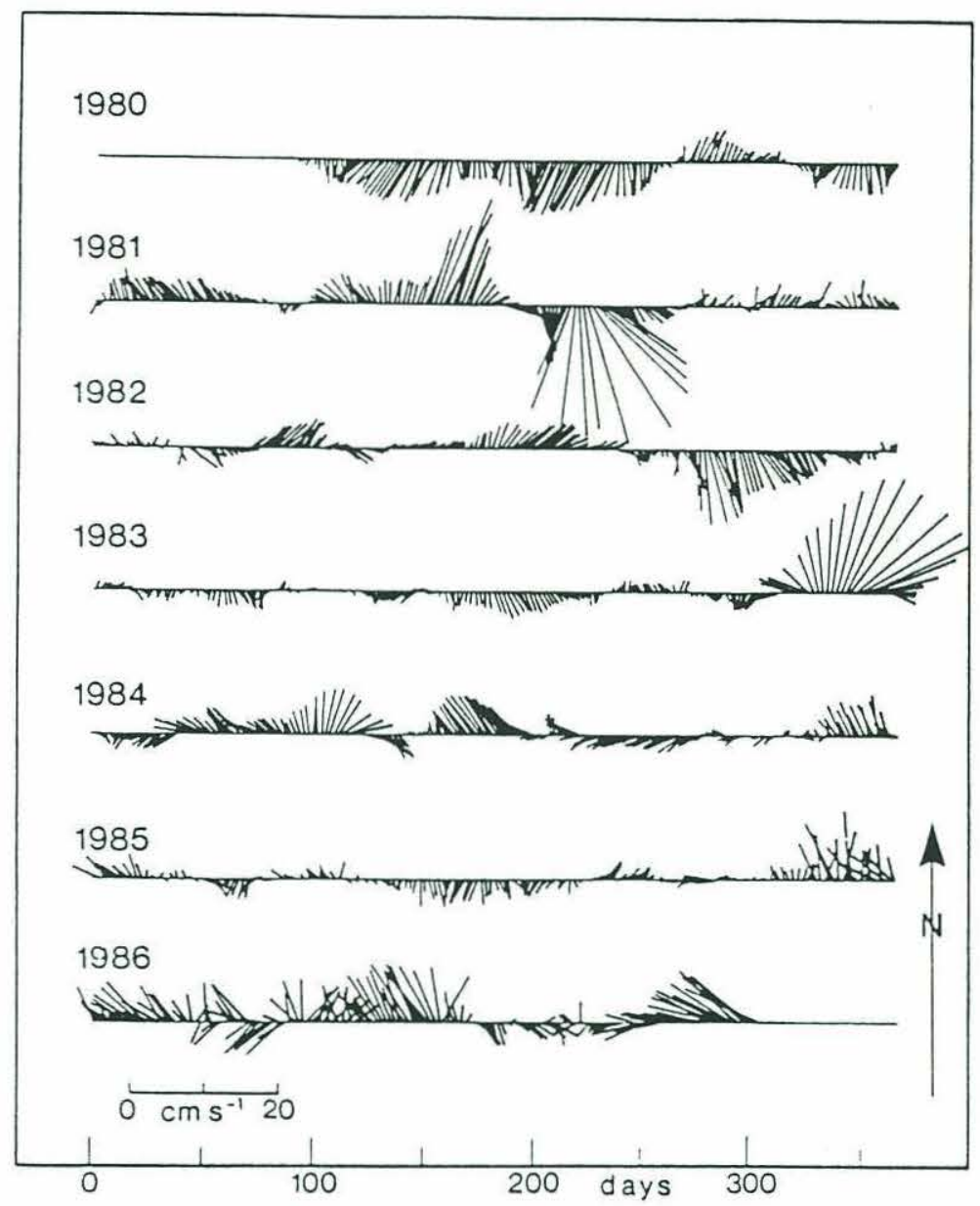

Figure 3-27. Current Vectors every 2 days at $1000 \mathrm{~m}$ near $33^{\circ} \mathrm{N} 22^{\circ} \mathrm{W}$ Reprinted from Zenk and Müller (1988, Fig. 2). 
sloshing in this area (Price et al., 1986). Armi and Stommel (1983) find the variability of baroclinic velocity shear over a two year period in the " $\beta$-triangle" area (centered at $27^{\circ} \mathrm{N} 32.5^{\circ} \mathrm{W}$ ) to be as large as the baroclinic shear itself. Thus, it appears that the synoptic circulation at thermocline depths varies from year-to-year in the area of our inversion (Schmitz et al., 1988).

\section{Summary and Conclusions}

We have demonstrated how a model ocean obtained in Chapter 2 based on a limited set of modes can be used to study the ocean circulation. The region of analysis is a $12^{\circ}$ square located towards the eastern edge of the subtropical gyre, situated over the Mediterranean Water tongue. Analysis of the modal model's hydrographic structure (temperature, salinity, geostrophic velocity) shows that they are consistent with a climatological data set (Levitus, 1982). This, again, indicates how only a limited number of modes are necessary to synthesize diverse measurements.

The absolute circulation is estimated using inverse methods. The flow in the upper thermocline $(\sim 500 \mathrm{dbar})$ is anticyclonic which reveals the eastern edge of the wind driven subtropical gyre. The circulation is southeastward into the northern part of the $12^{\circ}$ square, and is southwestward out of the region in the southern half. The anticyclonic flow quickly becomes weak with depth. Near the core of the maximum salinity anomaly ( $\sim 1200 \mathrm{dbar})$, the sense of the circulation is cyclonic, flowing around the Mediterranean salt tongue with little flow along the tongue's axis. This cyclonic circulation at mid-depth is consistent with the interpretation of Reid (1978), and Behringer and Stommel (1980), but is at odds with the common notion that the water should be "spreading" westward in the Mediterranean tongue (i.e. the core layer method, Wüst, 1935). However, in the presence of both advection and mixing, the direction of

spread does not necessarily coincide with the direction of mean flow (e.g. Richardson and Mooney, 1975). The results from the present analysis would indicate that mixing 
plays an essential role in the westward spread of the salinity anomaly. Perhaps the recently observed lenses of relatively undiluted Mediterranean Water embedded in the Mediterranean tongue (Armi and Zenk, 1984) are essential in maintaining the westward extension of the tongue itself. Armi and Stommel (1983) estimate a substantial salt flux divergence associated with such anomalies. On the other hand, we should note that there is nothing in the hydrographic structure in the region itself that rules out the possibility that the westward extending tongue is a static feature. It is only with the knowledge of the boundary condition, i.e. Mediterranean outflow from the Strait of Gibraltar, that we are forced to consider otherwise.

We also find that mass is conserved without invoking any mixing, suggesting diapycnal mass flux to be unimportant in the mass balance inside the Mediterranean salt tongue. (At least on the scales we have analyzed.) However, the model and data do suggest that other processes possibly including mixing might be important at shallower depths $(\sim 500 \mathrm{~m})$.

This study has also been an exercise in how to systematically analyze density data to determine circulation over an entire region. The optimal procedure in determining absolute circulation is found to be a combination of the box model and the $\beta$-spiral method. The results from the $\beta$-spiral alone does not, in general, conserve property over a finite area. On the other hand, the box model becomes degenerate when applied over a small region. The two methods were applied simultaneously to determine the absolute circulation at every $2^{\circ}$ latitude and longitude inside the $12^{\circ}$ region.

The modal description has simplified aspects of the data set-up for the inverse model; e.g. mapping (Section 8.1, Chapter 2), and decisions on the numbers and depths of layer boundaries (Section 4.1). However, the actual inversion is carried out in the same way as with classical data sets; namely, it is based on temperature, etc., on standard depths. The inversion still involves solving many similar equations at neighboring boxes and grid points. There are still redundancies in the inversion, which 
do not capitalize on the underlying simplicity. Ideally one would like to determine circulation directly from the modal coefficients so as to reduce the mathematics of the inversion itself. This problem is left for future examination. 


\section{CHAPTER 4 \\ Meridional Circulation and Mixing of a Warm-Core Ring Determined by Inverse Methods}

\section{Introduction}

The general theme of this thesis is the study of the use of systematic mathematical techniques for determining the ocean circulation from classical hydrographic data. There are many aspects of such problems, ranging from questions of how to handle data to the mathematics of the models once the data have been reduced to useful form. Most of this thesis has been devoted to the former question - i.e. data reduction so as to make it most useful and informative. In the last chapter, we began the exploration of the second type of question - in particular how to carry out inversions on small regional scales, having shifted the focus from the entire North Atlantic to a small section of the eastern basin.

In this chapter, we pursue this second theme, the application of inverse methods, in general, to oceanographic circulation problems. But here the scale is reduced even further - to that of a single Gulf Stream ring. Most inverse calculations have hitherto been applied to larger scales and to quasi-geostrophic balances. We will show, among other results, that the methods can be applied to very different scales and dynamical balances.

Gulf Stream and Kuroshio rings exist for many months and even years (Brown et al., 1986; Tomosada, 1986). During periods when the rings are not directly interacting with the western boundary currents which gave rise to them, the decay processes appear to be slow, involving physics which remains somewhat obscure. One form of decay is radiation by Rossby wave dispersion (McWilliams and Flierl, 1979; Mied and Lindemann, 1979). But the rings are observed to mix with their surroundings, and 
these mixing processes are likely to be important in understanding the decay process at least in part.

Cheney and Richardson (1976) gave one of the first views of a decaying ring. They find height anomaly of the isotherms in a cold core ring to gradually decrease at a rate of about $1 \mathrm{~m} /$ day. The available potential energy decreased at a rate close to $1 \mathrm{GW}$, which gives a life expectancy of 2.5 years for their cold core ring. Olson et al. (1985) have found a similar evolution in a warm core ring with energy decaying at $0.1 \mathrm{GW}$. These slow changes of a freely propagating ring contrasts with the rapid evolution caused by interactions with the Gulf Stream. Joyce et al. (1984) observed a $60 \%$ decrease in available potential energy in a warm core ring in just 10 days as a result of such an interaction. This energy loss is at $\sim 7.7 \mathrm{GW}$ which is an order of magnitude larger than during slow evolution.

Schmitz and Vastano $(1975,1977)$ have estimated meridional circulation from the changes in temperature structure of a cold core ring in their parametric studies of the mixing coefficients. This circulation has an inflow in the upper and lower parts of the ring with an outflow at intermediate depths.

A similar analysis for a case of a warm core Gulf of Mexico eddy resulted in similar streamlines but with an opposite sense of flow (Schmitz and Vastano, 1976). However, there was no attempt simultaneously to balance the vorticity change in the ring, so the estimated circulation is not necessarily consistent with the azimuthal velocity changes.

Flierl and Mied (1985) studied frictionally driven circulation of a ring in a theoretical model by solving the angular momentum equation together with density changes. They found a single-celled meridional circulation driven by horizontal mixing whose sense of circulation depends on the Prantl number, $\operatorname{Pr}=\nu_{h} / \kappa_{h}$. In the viscous case, $\nu_{h}>\kappa_{h}$, the flow is radially outward in the upper part of the ring and inward at depth with upward flow at ring center. The sense of circulation is opposite in the diffusive 
case, $\nu_{h}<\kappa_{h}$. They also find that vertical mixing is ineffective in driving meridional circulation.

The present study is directed at analysis of angular momentum changes and temperature and salinity variations in an observed warm core ring, so as to estimate the meridional circulation and mixing. The particular ring being studied is warm core ring $82 \mathrm{~B}$, which was investigated extensively during the Warm Core Rings Experiment (Warm Core Rings Executive Committee, 1982). Ring 82B was formed in February 1982 from a northward meander of the Gulf Stream. The ring propagated at speeds of several $\mathrm{cm} / \mathrm{s}$ southwestward along the continental slope. The ring arrived near Cape Hatteras in July, where interactions with the Gulf Stream later lead to absorption of the ring with this parent current. A detailed event chronology of ring $82 \mathrm{~B}$ can be found in Evans et al. (1985). During April, June, and August, intensive multiship surveys of ring $82 \mathrm{~B}$ were undertaken. While the April and June period is characterized by the slow evolution of the warm core ring, the period between June and August is marked by a rapid decrease of ring volume and energy from interactions of the ring with the Gulf Stream and topography (Olson et al., 1985). Besides the general relaxation of the thermocline's height anomaly, the April to June period is also characterized by the set up of the seasonal thermocline, which caps the exposed thermostad in April (Schmitt and Olson, 1985). The present analysis focuses on the ring evolution between April and June.

We first describe some of the relevant structural changes of ring $82 \mathrm{~B}$ in the meridional plane in Section 2. Data are azimuthally averaged and mapped radially along isopycnals. In Section 3, we formulate a diagnostic model to estimate the circulation and mixing which would account for those changes seen in the ring. The model is solved as a linear inverse problem. The result of this model is discussed in Section 4. In Section 5, we further examine a series of models in which mixing is modeled differently. Section 6 gives a summary of our findings. 


\section{Structure of Ring 82B}

\subsection{Explanation of Data}

During the field experiments in April and June, some 30 CTD stations were occupied in the ring (Figure 4-1, reproduced from Smith and Baker, 1985) and near surface current was measured continuously throughout these observations by a shipboard acoustic . doppler current meter (Joyce and Kennelly, 1985). These two data sets form the basis of our analysis. To best describe the changes of the ring's structure, these data are analyzed relative to ring center which is determined from a combination of satellite sea surface temperature measurements, CTD, XBT and drifter data (Hooker and Olson, 1984). In the vertical, we use a density coordinate to follow the ring's adjustment of vertical structure as a result of frictional spin down, change in latitude, and surface stratification. The set of potential density surfaces that we choose to analyze are listed in Table 4-1. These levels were chosen so as to resolve the main structural features of the ring, namely the thermostad and thermocline. As the thermostad is also a near pycnostad, we could only identify 3 levels that delineate it. The levels in the pycnocline are more or less equally spaced down to $1000 \mathrm{~m}$.

Finally, we azimuthally average the data and concentrate on the structural variations in the vertical and radial plane. This mapping was performed radially along each separate isopycnal using objective mapping (Bretherton et al., 1976). The statistics that are used in mapping are:

- A Gaussian data correlation function with a $50 \mathrm{~km}$ correlation distance.

$\circ$ A signal to noise ratio of 1.0 .

These statistics are chosen somewhat arbitrarily, but comparison with the data shows that the above statistics are not grossly unrealistic. The same statistics were used to map pressure (dbar), temperature $\left({ }^{\circ} \mathrm{C}\right)$, salinity (practical salinity units), dynamic

height anomaly $\left(\mathrm{m}^{2} / \mathrm{s}^{2}\right)$, and azimuthal velocity $(\mathrm{m} / \mathrm{s})$ at $28 \mathrm{~m}$ depth (measured by 


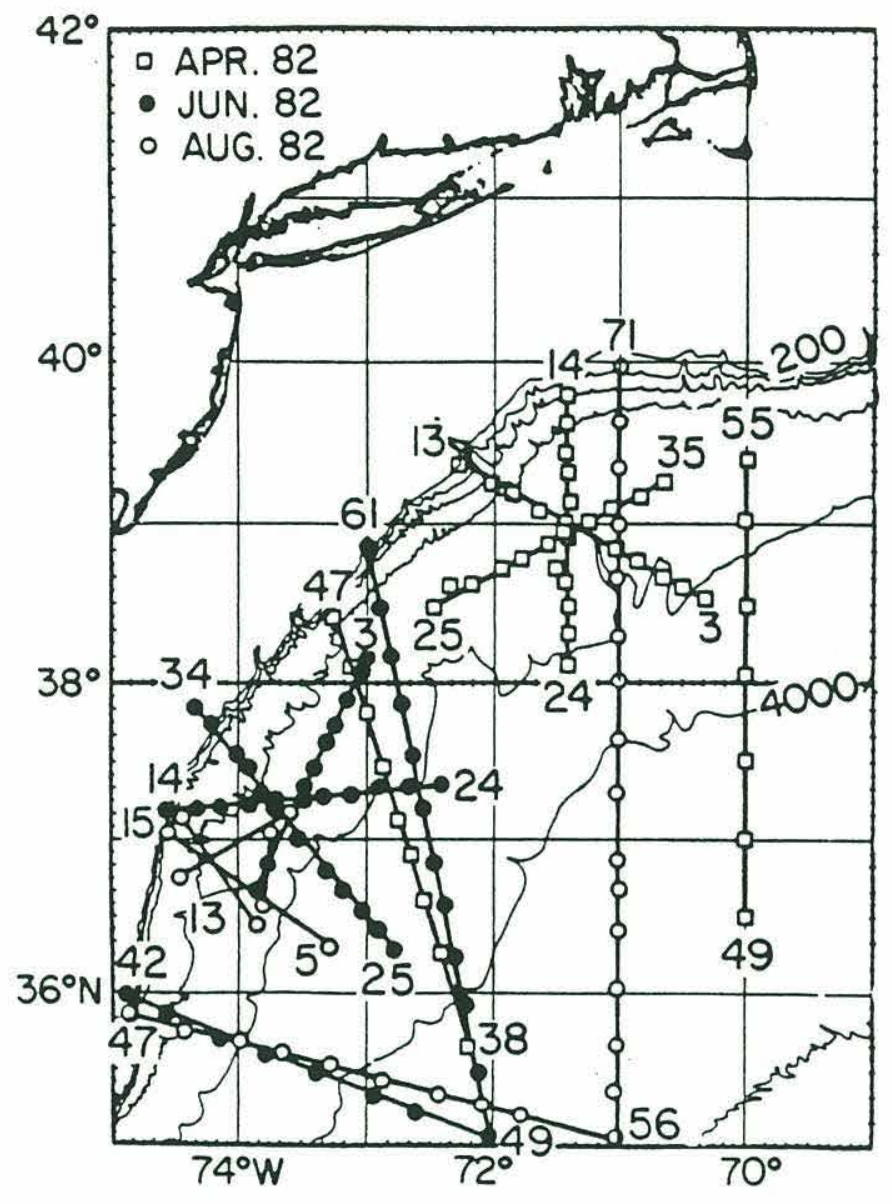

Figure 4-1. CTD Station Locations

This figure is reproduced from Smith and Baker (1985, Fig. 1). The stations in asterisk shape (April and June) are the ones used in the present study. 


\begin{tabular}{|r|l|r|r|l|}
\hline level & $\sigma_{\theta}$ & $\mathrm{P}^{*}(\mathrm{dbar})$ & $\mathrm{T}^{*}(\mathrm{deg} \mathrm{C})$ & Comment \\
\hline 0 & 26.6 & $31.5 \dagger$ & $16.3 \dagger$ & within the seasonal thermocline \\
1 & 26.77 & 24.6 & 15.9 & top of pycnostad \\
2 & 26.8 & 287.7 & 15.6 & middle of pycnostad \\
3 & 26.83 & 414.8 & 14.9 & base of the pycnostad \\
4 & 26.9 & 435.7 & 13.9 & \\
5 & 27.0 & 463.8 & 12.6 & \\
6 & 27.1 & 503.3 & 11.3 & \\
7 & 27.2 & 543.1 & 10.0 & \\
8 & 27.3 & 588.9 & 8.9 & \\
9 & 27.4 & 630.6 & 7.8 & \\
10 & 27.47 & 661.6 & 7.1 & \\
11 & 27.53 & 692.7 & 6.6 & \\
12 & 27.58 & 726.2 & 6.1 & \\
13 & 27.62 & 765.1 & 5.7 & \\
14 & 27.65 & 805.9 & 5.4 & \\
15 & 27.67 & 841.0 & 5.2 & \\
16 & 27.685 & 872.2 & 5.1 & \\
17 & 27.7 & 911.2 & 4.9 & \\
18 & 27.71 & 945.5 & 4.8 &
\end{tabular}

* The values are the average between April and June at ring center of the mapped estimate.

$\dagger$ The value is for June.

Table 4-1. Isopycnals on which Properties are Mapped Also shown are the pressures and temperatures on each isopycnal at ring center (average of April and June values). 
the acoustic doppler current meter). All of these variables are mapped for April and June observations.

Figure 4-2 is an example of the mapped isopycnal depth for the top of the thermocline. Mapping is done with data as a function of radius $(r>0)$. To assure azimuthal symmetry and a no cusp at ring center $(\partial / \partial r=0)$, mapping is performed between $-100 \mathrm{~km}<r<100 \mathrm{~km}$, with data used in mapping made symmetric about $r=0$. That is, data values at $r<0$ in Figure 4-2 (the crosses) are mirror images of data at $r>0$. Mapped estimates are made every $5 \mathrm{~km}$ from ring center to $100 \mathrm{~km}$ radius. The same procedure is used for all variables except velocity which is projected antisymmetrically since it is a vector component that changes sign across ring center. This procedure guarantees the velocity at ring center to be 0 . In the remainder of this section, we present a description of the ring's structure from density levels 1 to 18 (Table 4-1). Discussion of the first isopycnal is excluded as this surface only exists in June. The mapped radial component of velocity obtained from the current meter is not discernible from zero, and will not be discussed either.

\subsection{Depth of Isopycnals}

Figure 4-3 shows the depth of the 18 isopycnals in April. The top isopycnal shows the doming of the thermostad towards ring center, while all the other isopycnals below deepen. The distance between the isopycnals in the thermocline are nearly uniform with radius except for the top-most layer where the isopycnal distance is thinner towards ring center. The structure in June is almost indistinguishable to the eye from that in April.

The depth differences of the isopycnals in the thermocline between these two months are shown in Figure 4-4. All isopycnals become shallower in June. There is a tendency for the outer portion of the ring to rise more rapidly than regions near ring center. This difference in the rate of rise is larger at deeper isopycnals which would 


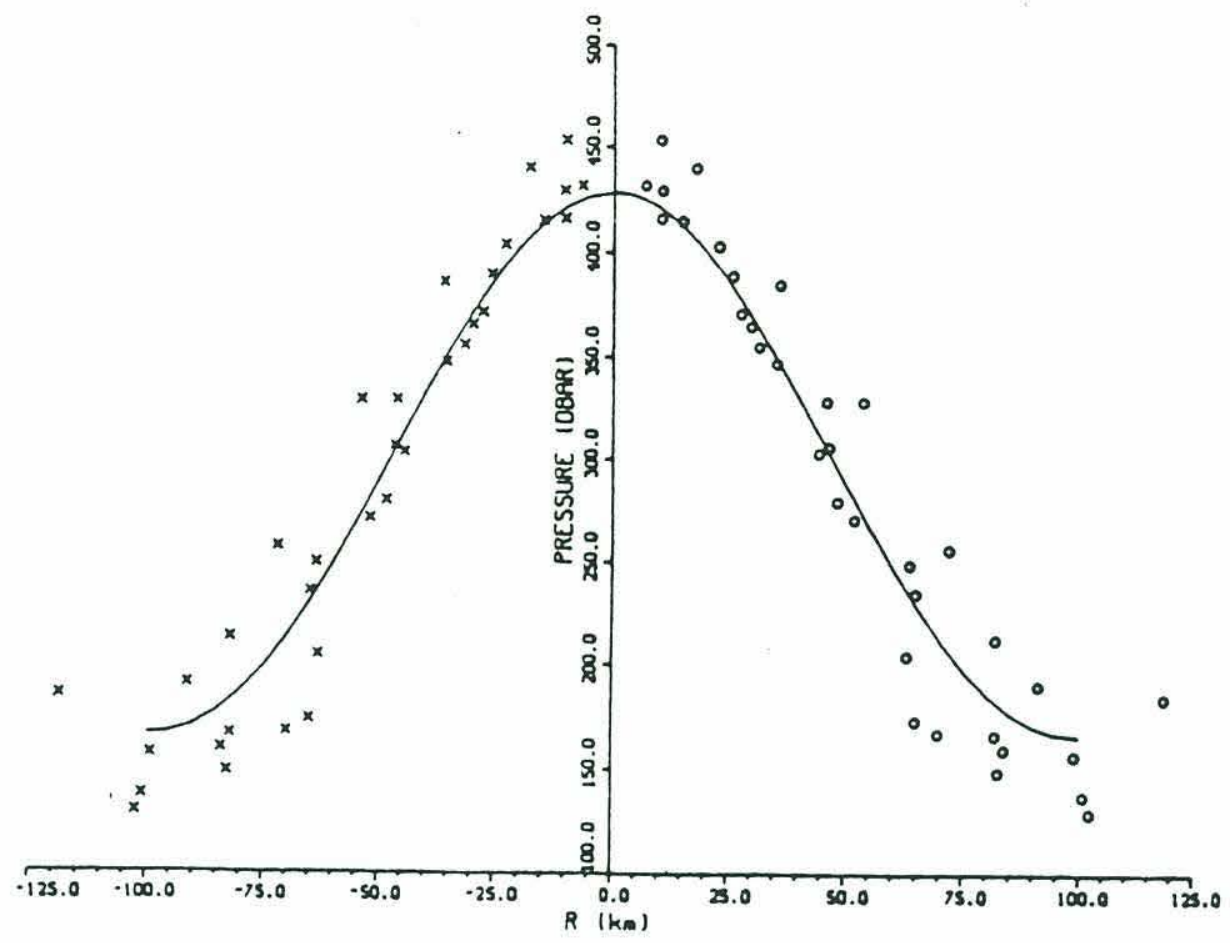

Figure 4-2. Mapped Depth (dbar) of $\sigma_{\theta}=26.9$

The circles are data plotted as a function of radius $(r>0)$. The crosses at $r<0$, which are also used in mapping to assure radial symmetry, are symmetric projections of the circles.

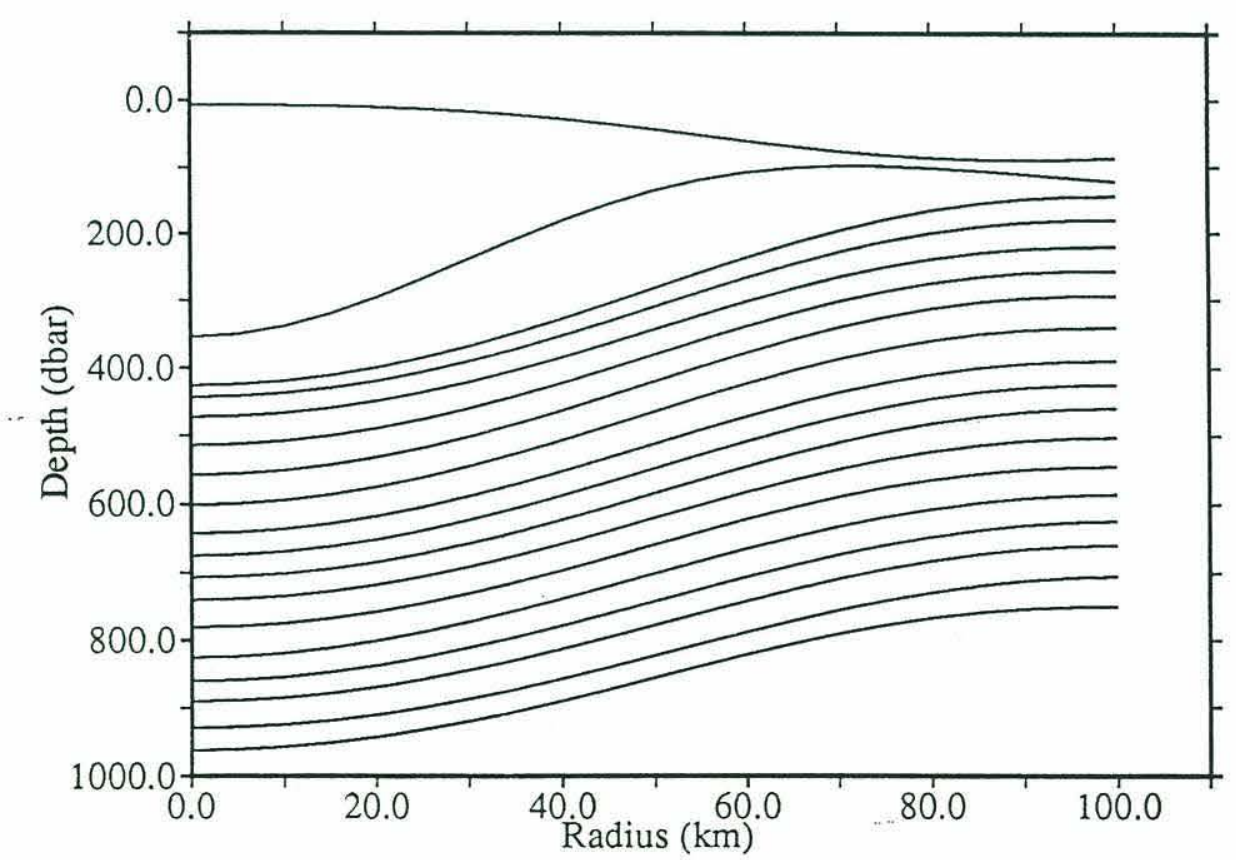

Figure 4-3. Isopycnal Depths (dbar) in April 


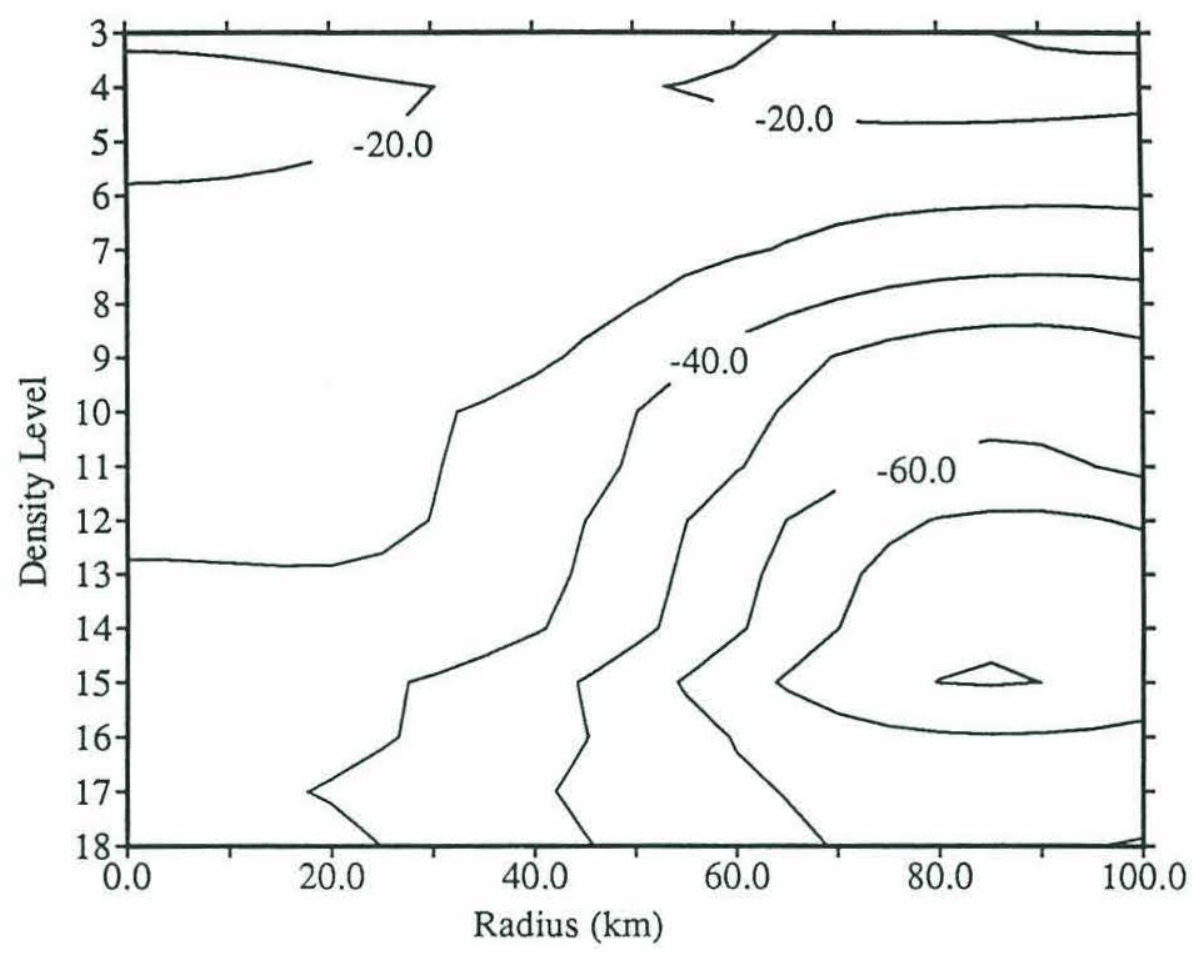

Figure 4-4. Depth Changes (dbar) of Thermocline Isopycnals Negative values indicate isopycnals becoming shallower from April to June.

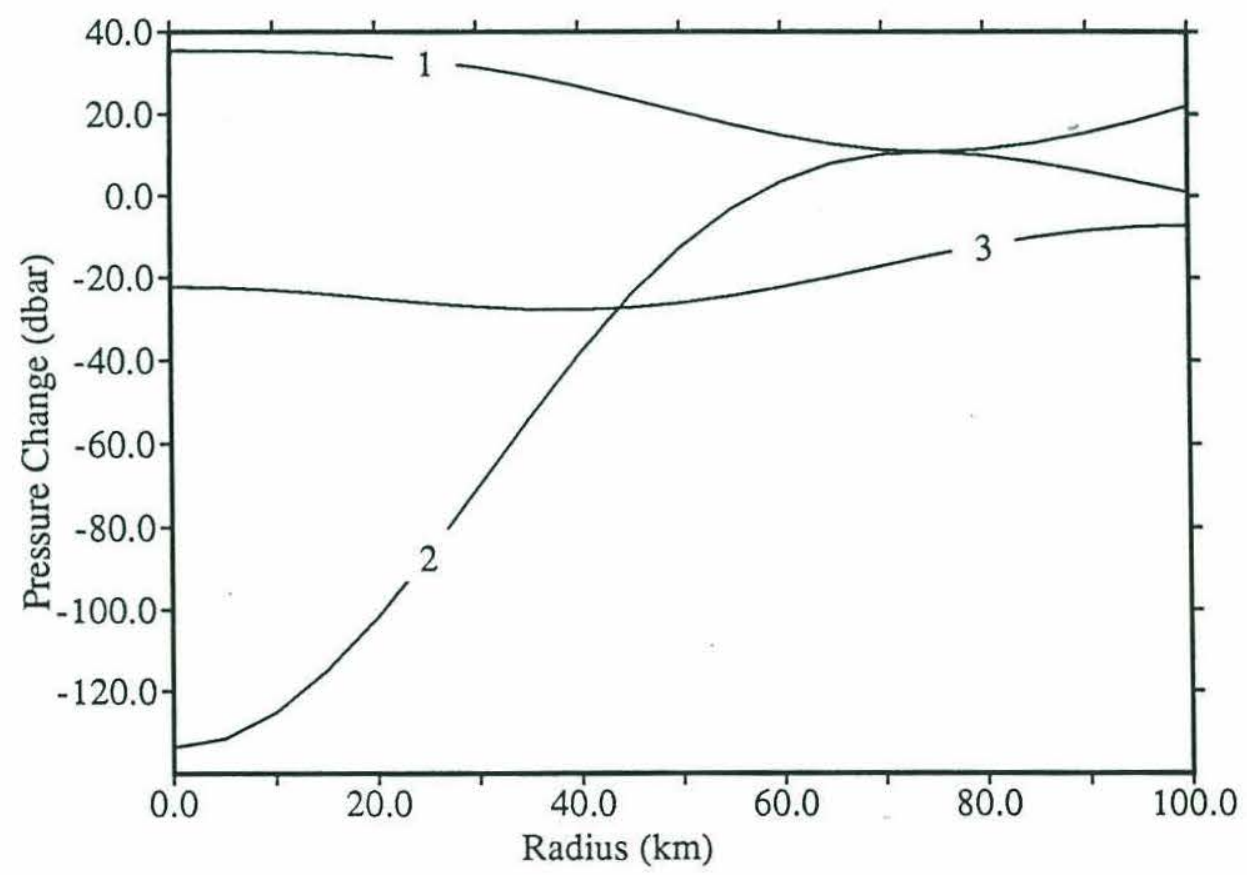

Figure 4-5. Depth Changes (dbar) of Thermostad Isopycnals 
mean strengthening of the stratification in the thermocline. Isopycnal movements of the thermostad are shown in Figure 4-5. Contrary to other isopycnal surfaces, the top isopycnal of the thermostad (level 1) deepens about $30 \mathrm{~m}$ during this period, which is the result of the seasonal thermocline setting up in early May (Schmitt and Olson, 1985). The mid-thermocline isopycnal (level 2) rises rapidly at ring center and deepens beyond $55 \mathrm{~km}$.

The errors of isopycnal depths and the other properties described below in this section are tedious to evaluate and unlikely to give further insight, but in general one contour interval in the figures of the temporal changes (two, in the case of derived quantities such as potential vorticity) is probably a reasonable estimate of these errors.

\subsection{Potential Temperature}

Potential temperature along isopycnals in April is shown in Figure 4-6. (Salinity has nearly the same pattern by definition of an isopycnal, and so it will not be discussed.) A strong radial gradient exists in the thermostad. This gradient is as large as $2^{\circ} \mathrm{C}$ from the center to $100 \mathrm{~km}$ radius, which contrasts the warm water of the Sargasso Sea/Gulf Stream origin in the core of the ring to the surrounding colder Slope Water (Joyce, 1984). The radial contrast continues through the top parts of the pycnocline. In fact, although small, we can detect this monotonic radial temperature gradient down to about the 13th isopycnal.

The temperature changes from April to June in the upper parts of the ring are displayed in Figure 4-7. Along the top of the thermostad (level 1) near ring center, potential temperature decreases by almost a quarter of a degree while at larger radii it increases about the same amount. The decrease at ring center is probably related to the air-sea interactions that set up the seasonal thermocline. Atmospheric heating has increased the temperature in the surface waters, making the water less dense. This heat is carried down by mixing which causes the isopycnal to sink into the water 


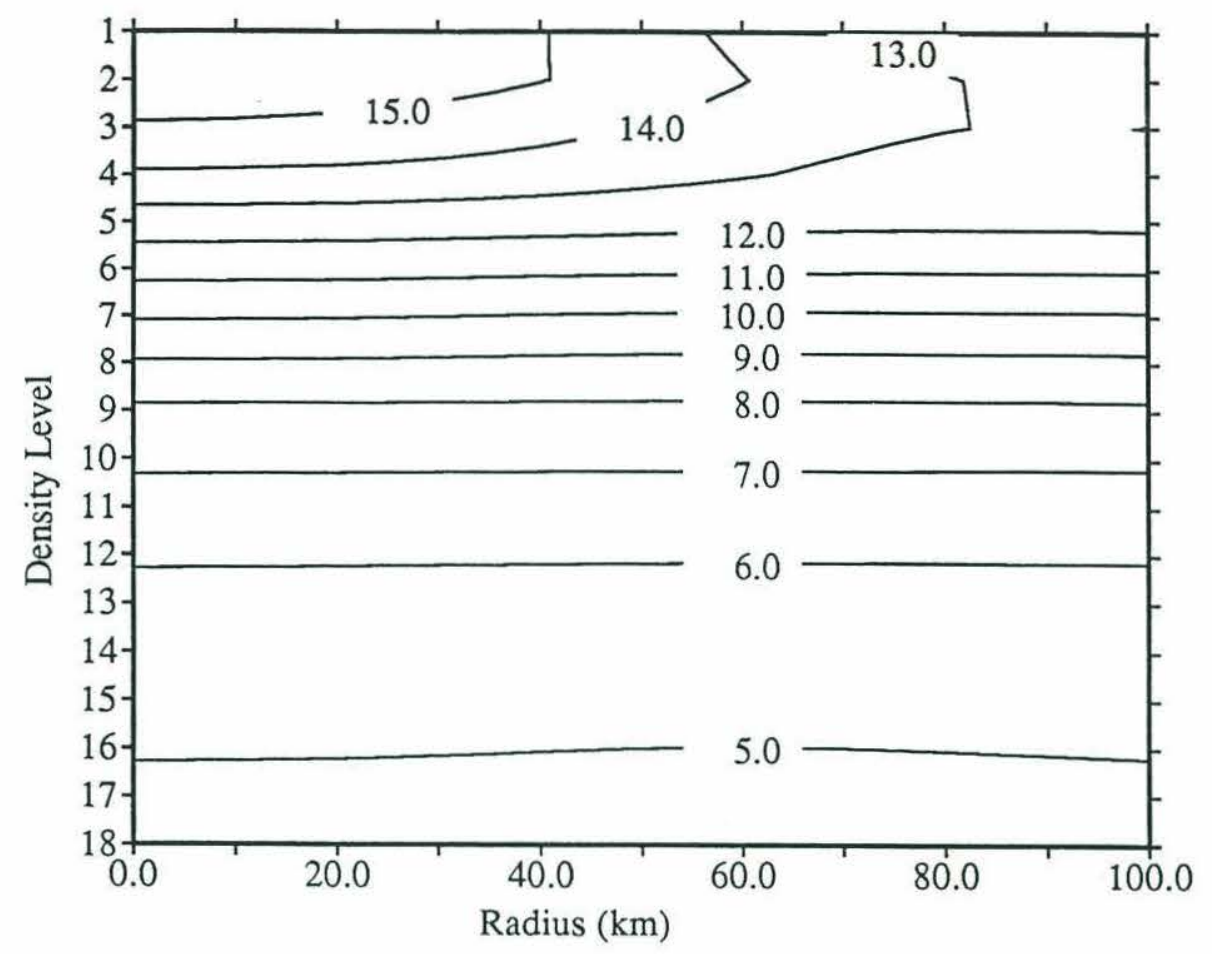

Figure 4-6. Temperature $\left({ }^{\circ} \mathrm{C}\right)$ Contour in April Axes are isopycnal levels (Table 4-1) and radius (km).

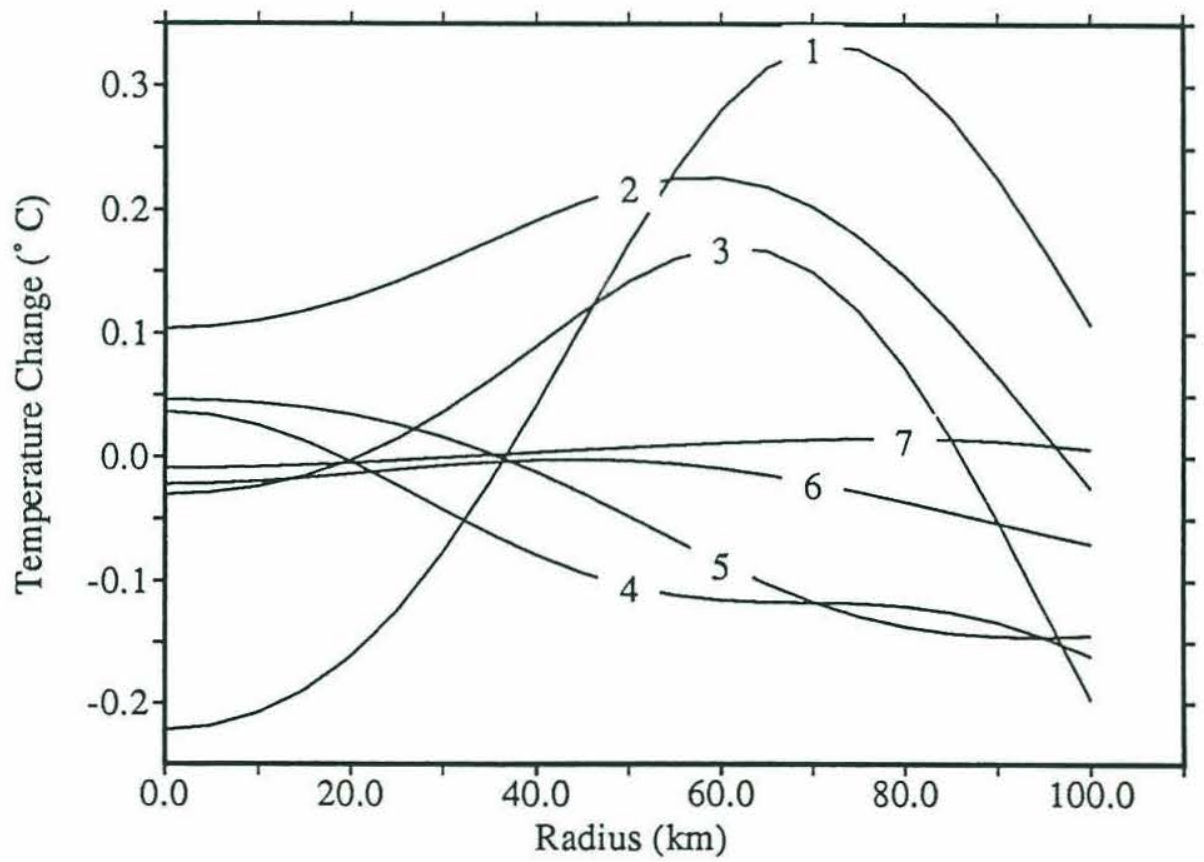

Figure 4-7. Temperature Change $\left({ }^{\circ} \mathrm{C}\right)$ in the Thermostad and Upper Thermocline The number on each curve is the isopycnal level given in Table 4-1. 
column, leading us to see different water parcels on the same isopycnal. The increase in temperature is maximum at $75 \mathrm{~km}$. This same tendency of maximum temperature increase at large radius is also seen on the other two isopycnals of the thermostad (levels 2 and 3). At the top of the thermocline (levels 4 and 5), temperature decreases more towards the ring's outer edge than at center where there is a slight temperature increase beneath the radii where the bottom of the thermostad decreases. Temperature at other deeper isopycnals has increasingly smaller changes.

\subsection{Azimuthal Velocity and Angular Momentum}

Azimuthal velocity, $v$ (positive cyclonic), can be calculated at depth using the gradient wind balance and the near surface velocity of the acoustic doppler current meter as a reference surface (Joyce, 1984). The gradient wind balance in pressure and density coordinates is,

$$
\begin{aligned}
\frac{v^{2}}{r}+f v & =\left.g z_{r}\right|_{p} \\
& =\frac{1}{\rho_{0}}\left(\left.p_{r}\right|_{\sigma}-p_{\sigma} \frac{z_{r}}{z_{\sigma}}\right) .
\end{aligned}
$$

The absolute dynamic topography, $z$, can be calculated by integrating equation (41) radially using the measured near surface azimuthal velocity, and combining with the mapped relative dynamic height from the CTD data, we can solve the quadratic equation (4-2) for $v$ along each isopycnal. However, the right hand side of equation (4-2) is susceptible to noise which can make the solution of that equation become complex. The solution of the quadratic equation became complex on level 3 inside 25 $\mathrm{km}$ in April and level 4 inside $20 \mathrm{~km}$ in June. In both situations, velocity was linearly interpolated between the ring center, where azimuthal velocity is 0 , to the inner most radius where equation (4-2) was solvable.

These azimuthal velocities are shown in Figures 4-8a and 4-8b for April and June, respectively. The velocities increase radially to about $50 \mathrm{~km}$ and then level off towards 
a.

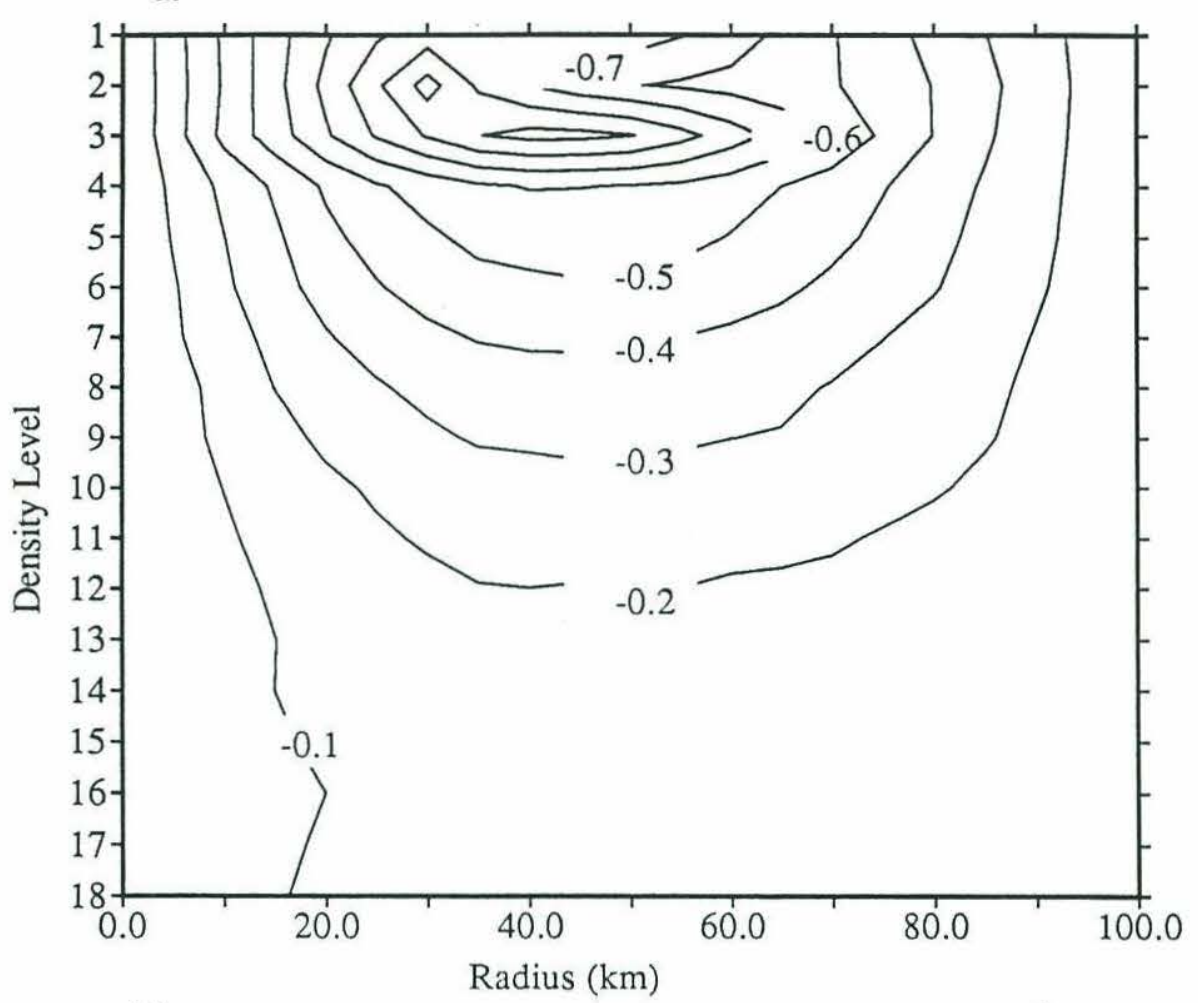

b.

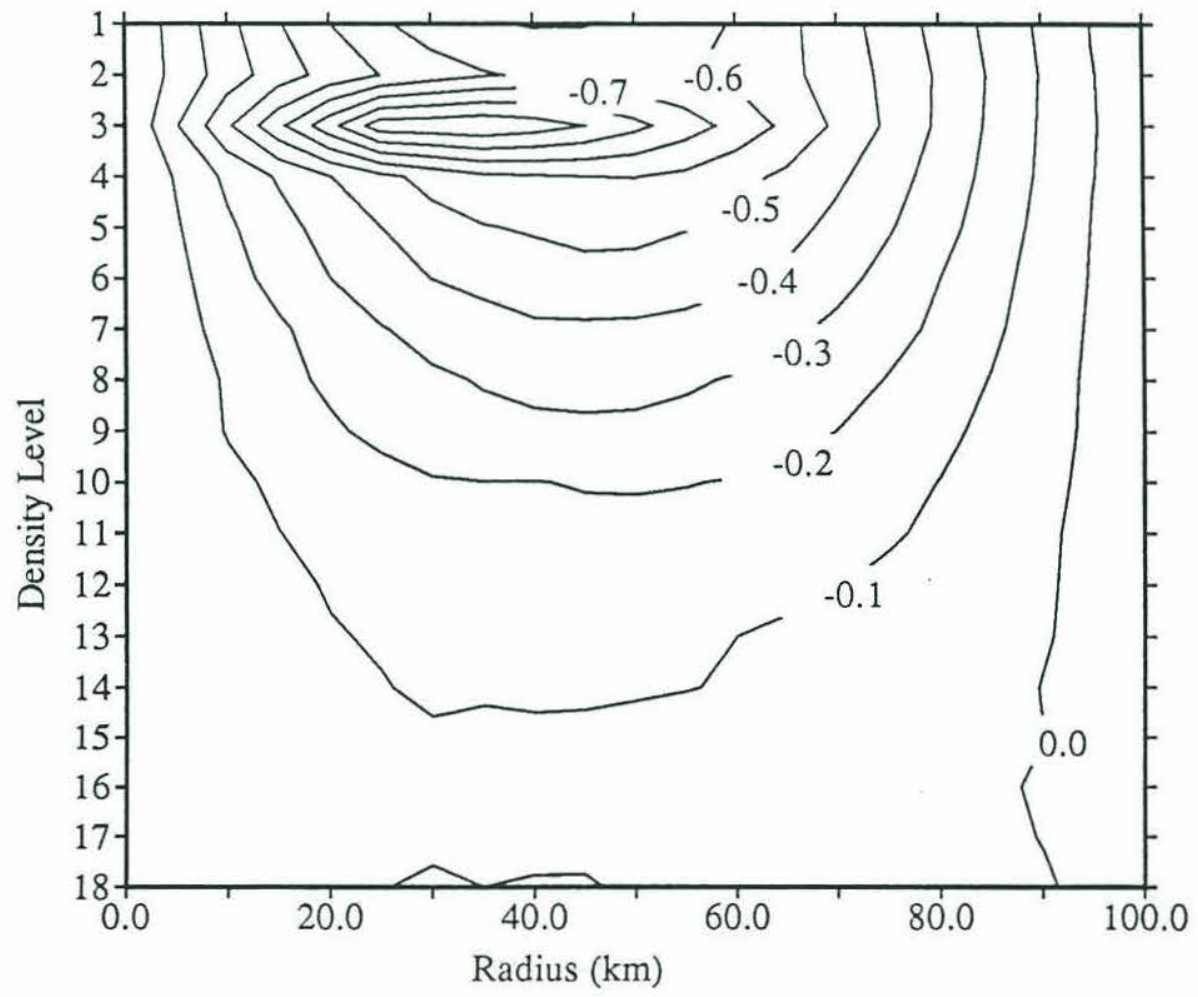

Figure 4-8. Azimuthal Velocity (m/sec) Contour as in Figure 4-6.

(a) April (b) June 
0 at $r \approx 100 \mathrm{~km}$. In the vertical, the velocity is maximum in the thermostad and gradually decreases through the thermocline. Inside the thermostad the maximum velocity is towards the base of the thermostad.

Temporal change in velocity is shown in Figure 4-9. The ring has spun down $(\Delta v>0)$ almost everywhere. This rate of spin down is almost depth independent at large radius $(r>50 \mathrm{~km})$. There is a large change at about $25 \mathrm{~km}$ in the middle and at the base of the thermostad. The spin down at the mid thermostad is related to the large movement of this isopycnal away from the velocity maximum at the bottom of the thermostad. The increase in swirl velocity at the base of the thermostad reflects the inward radial movement of the maximum swirl velocity at this depth.

As shown later in Section 3, angular momentum $(M)$,

$$
M=r v+\frac{1}{2} f r^{2}
$$

is conserved following a water parcel in the absence of friction. Angular momentum will later be used as one of the tracers to rationalize the ring's evolution. The angular momentum distribution in April is shown in Figure 4-10. The dominant structure is the second term in equation (4-3), and the ring's larger swirl is evidenced only by the slight tilt of the isolines towards larger radius and the "dent" in the profile at the base of the thermostad as a result of the large azimuthal velocity there.

The differences between April and June are shown in Figure 4-11. There is a general decrease in angular momentum between 40 and $90 \mathrm{~km}$ radii on almost all density surfaces, especially towards the top of the thermocline. On the other hand, despite the significant change in latitude, angular momentum remains nearly unchanged close to ring center and at the ring's edge $(\sim 100 \mathrm{~km})$. We can also see the angular momentum change at the bottom of the thermostad at $25 \mathrm{~km}$ associated with the large radial velocity change there. 


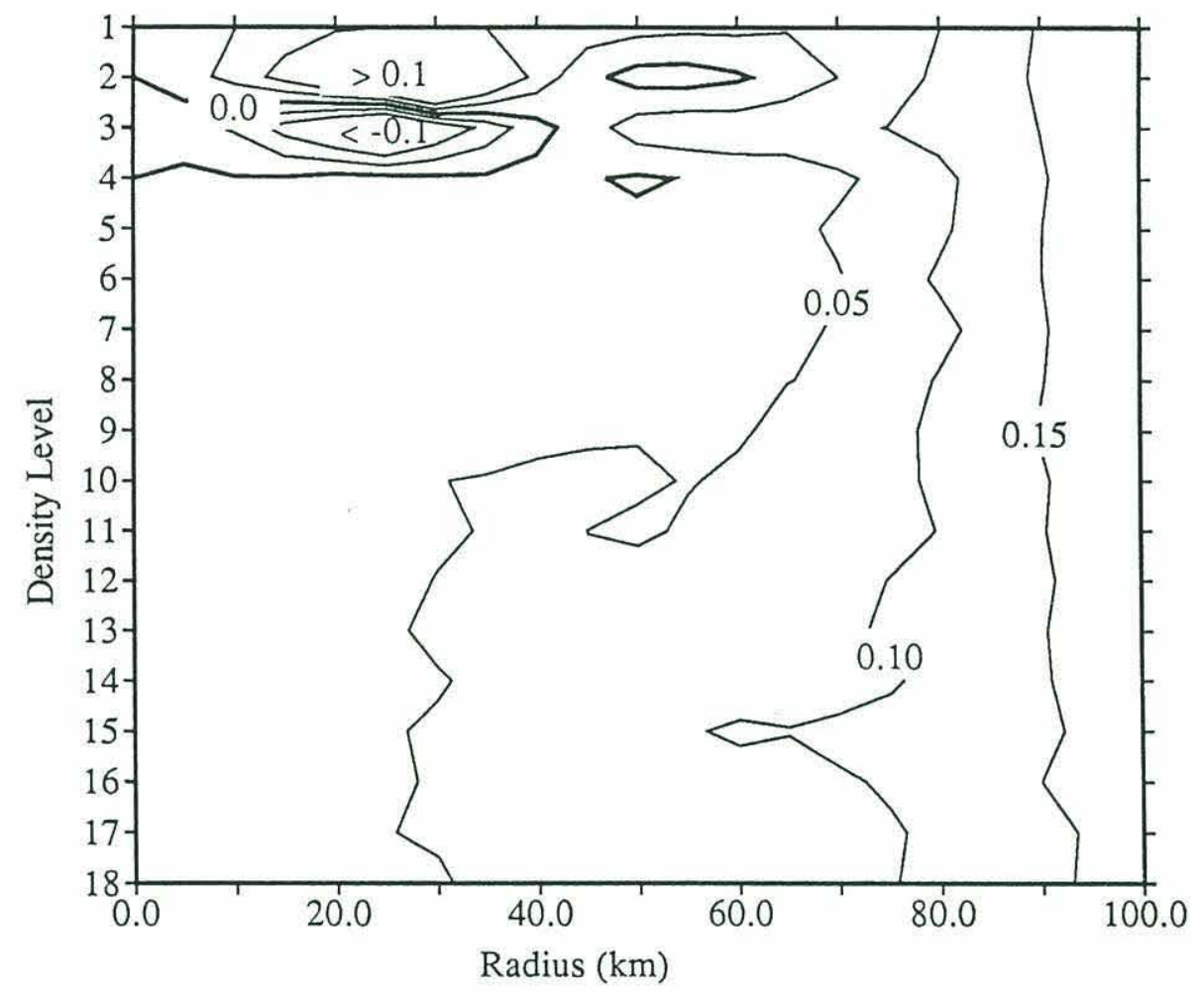

Figure 4-9. Azimuthal Velocity Change (m/sec) 


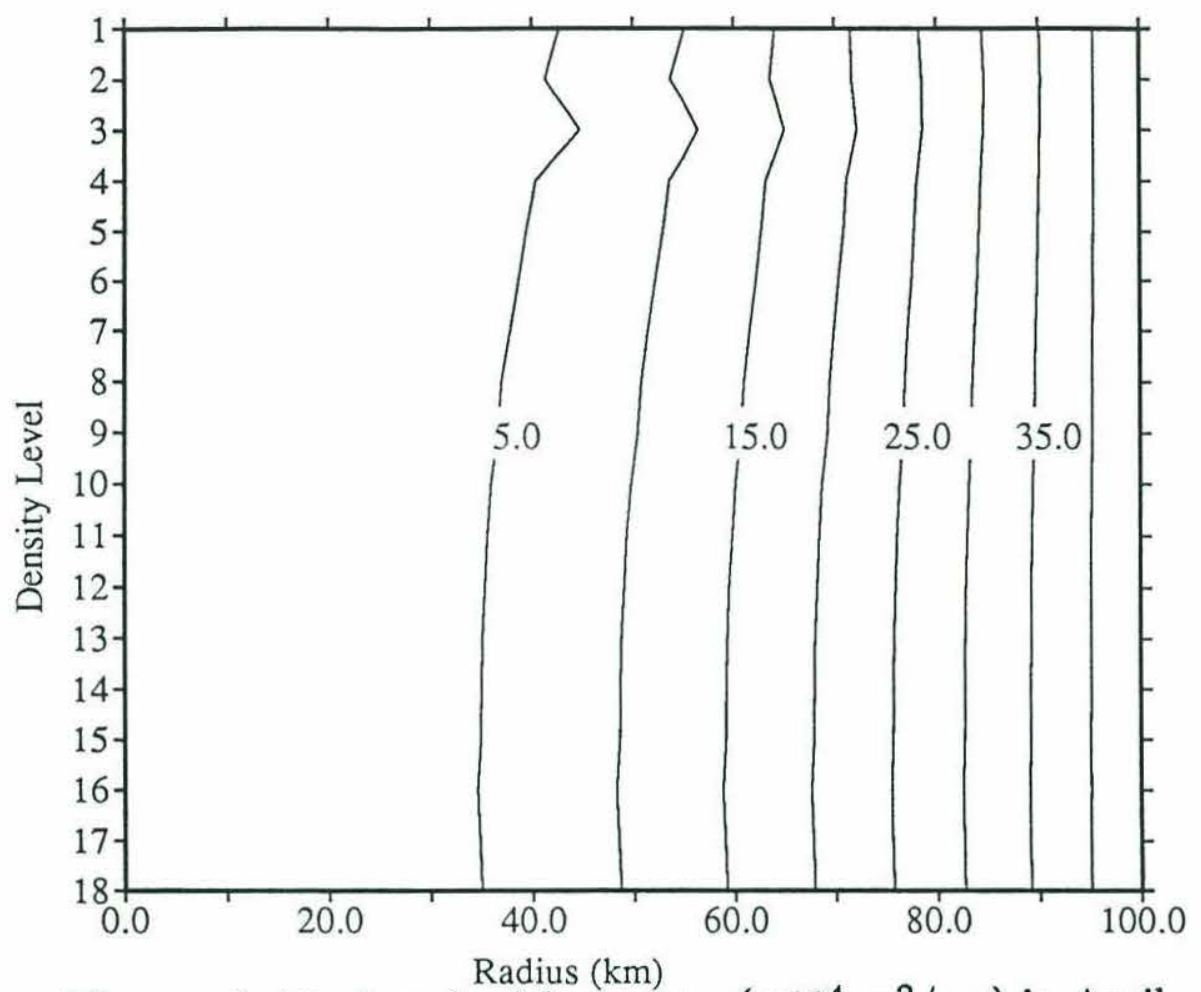

Figure 4-10. Angular Momentum $\left(\times 10^{4} \mathrm{~m}^{2} / \mathrm{sec}\right)$ in April

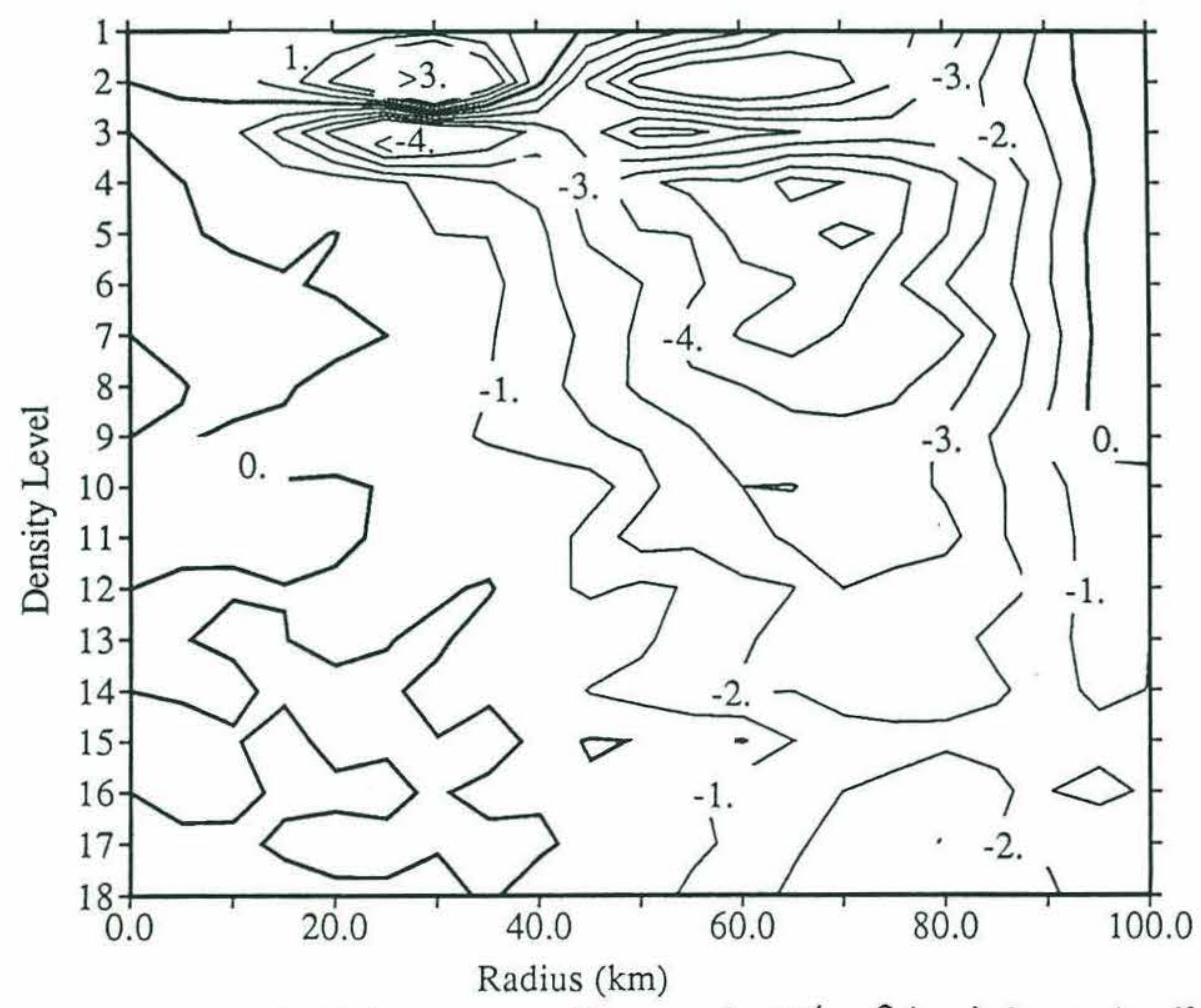

Figure 4-11. Angular Momentum Change $\left(\times 10^{4} \mathrm{~m}^{2} / \mathrm{sec}\right)$ from April to June. 


\subsection{Potential Vorticity}

Combining thickness between isopycnals and velocity estimates, we can calculate potential vorticity $(Q)$,

$$
Q=\frac{f+\zeta}{\Delta}
$$

where $\Delta=-\rho_{0} z_{\sigma}$ is thickness between isopycnals. The potential vorticity structure in April is shown in Figure 4-12. The thermostad is nearly constant in $Q$ both radially and vertically. This layer is separated by a large $Q$ gradient between the thermostad and the thermocline below and a strong radial gradient beyond $60 \mathrm{~km}$. The thermocline can be divided into two domains. In the upper part, $Q$ is relatively depth independent inside $50 \mathrm{~km}$, outside of which $Q$ increases. The deeper part of the thermocline, on the other hand, has large vertical gradients but little radial contrast. If constant potential vorticity can be interpreted as an indicator of the same water mass, the waters of the deeper thermocline are of the Slope Water origin since there is no radial contrast. On the other hand, the strong difference between the center and outer edges in the thermostad is consistent with the contrast between the Sargasso Sea origin water at ring center and the Slope Water at the ring's edge. The water at the upper thermocline is indicative of vertical mixing which causes the otherwise constant $Q$ on isopycnals to become vertically homogeneous, by bringing larger Slope Water values closer to the thermostad values.

The above interpretation of potential vorticity structure is consistent with the temporal changes shown in Figure 4-13. Despite the thinning of the thermostad and the rise of the thermocline, potential vorticity remains unchanged near ring center $(r<50 \mathrm{~km})$, throughout the thermostad and deep thermocline (see also the one layer calculation of Olson et al., 1985). These invariance of $Q$ indicate that the change in depth of the thermocline may primarily be the result of the ring adjusting to a smaller Coriolis parameter, $f$, as it moves to a lower latitude and not directly a result of spin down. On the other hand, there is an increase in $Q$ outside the $50 \mathrm{~km}$ radius in the 


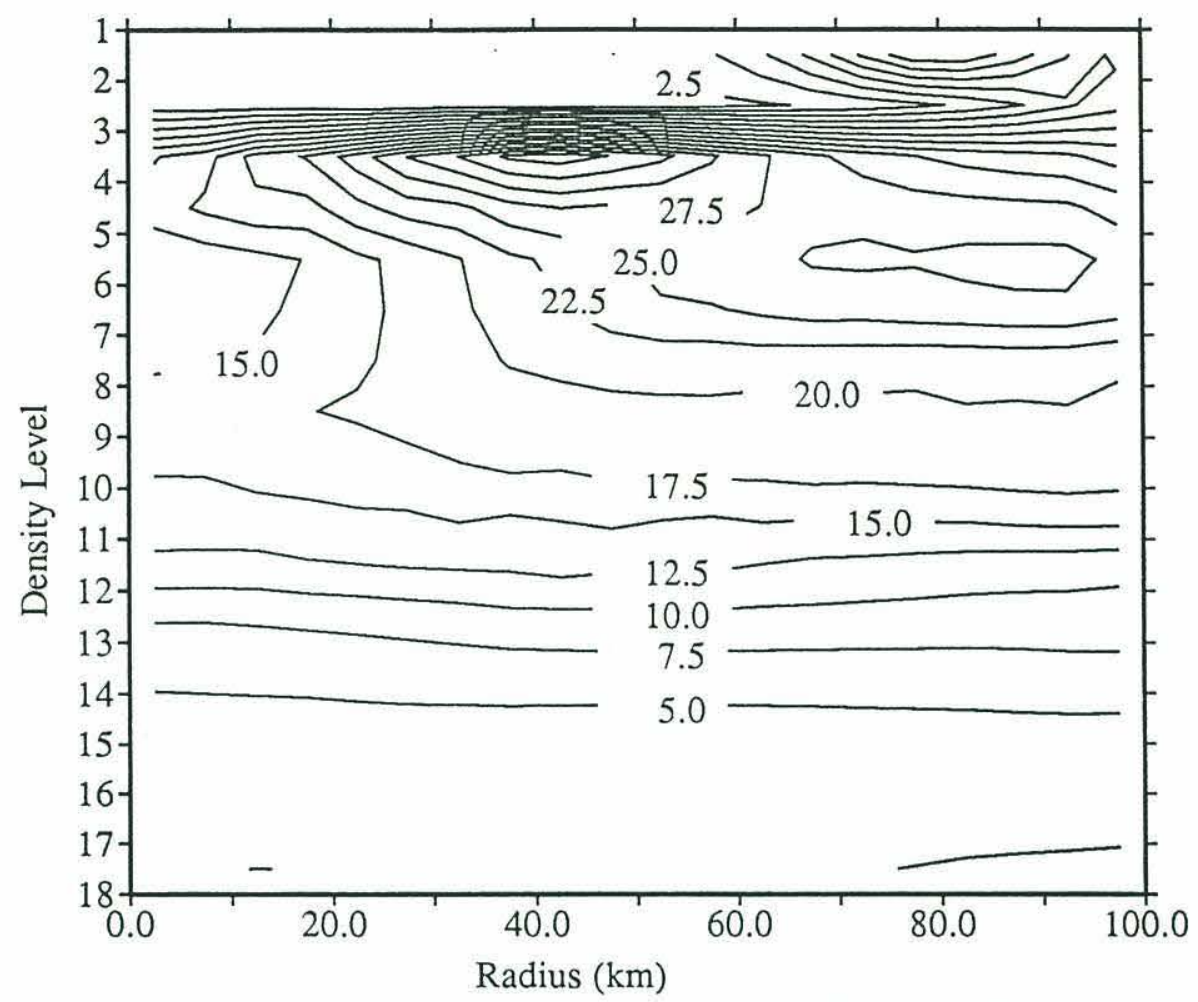

Figure 4-12. Potential Vorticity $\left(\times 10^{-11}(\mathrm{~ms})^{-1}\right)$ in April.

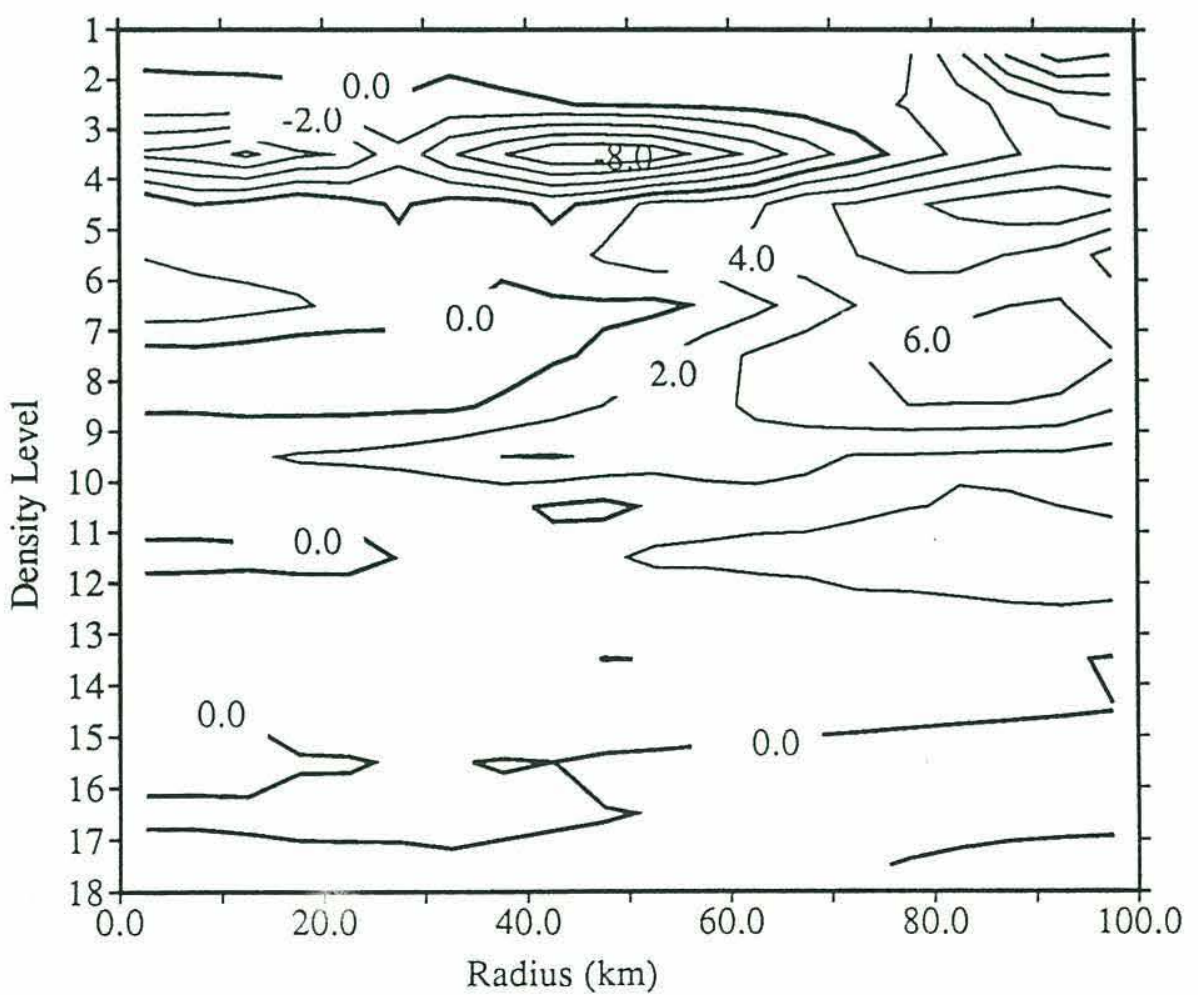

Figure 4-13. Temporal Change of Potential Vorticity $\left(\times 10^{-11}(\mathrm{~ms})^{-1}\right)$ 
upper thermocline and throughout the thermostad which is weakly depth dependent. The third layer (between level 3 and 4), which is the top most layer in the thermocline right beneath the thermostad shows a somewhat different behavior. Although it also shows an increase at large radius, it consistently decreases its value inside $70 \mathrm{~km}$. If the density coordinate allows us to follow water parcels in time, potential vorticity should also be conserved and the changes could only occur by mixing. The increase at large radii is consistent with lower $Q$ Sargasso Sea origin water at the ring's core being mixed horizontally with the higher $Q$ Slope Water. The decrease beneath the thermostad could be the result of the upper thermocline (larger $Q$ ) being mixed with the thermostad water (lower $Q$ ) above.

\subsection{Summary}

Potential vorticity and other changes show that the ring slowly evolved while still maintaining its contrast with the surrounding waters. Some structure changes, like the rise of the thermocline near ring center, are likely to be an adjustment to a lower latitude, but the nature of other changes strongly suggests that mixing had a role in the ring's evolution. The mixing and circulation associated with the structural changes are the topic of the following sections.

\section{The Model}

We now construct a simple diagnostic model to estimate what circulation and mixing underlie the structural changes in the meridional plane of warm core ring $82 \mathrm{~B}$. The thinning of the thermostad and other movements of isopycnals indicate movement of mass inside the ring. Mass conservation in an azimuthally symmetric ring in cylindrical coordinates is,

$$
\frac{1}{r} \frac{\partial}{\partial r}(r u)+\frac{\partial w}{\partial z}=0
$$


Vertical velocity can be written in terms of variables in the density coordinate $(\sigma)$ as,

$$
w=\frac{\partial z}{\partial t}+u \frac{\partial z}{\partial r}+\omega \frac{\partial z}{\partial \sigma}
$$

where $z$ is the height of an isopycnal and $\omega$ is the rate of density change following a water parcel;

$$
\omega=\frac{\mathrm{d} \sigma}{\mathrm{d} t} .
$$

Using this, equation (4-5) is rewritten in density coordinates as,

$$
\frac{\partial h}{\partial t}+\frac{1}{r} \frac{\partial}{\partial r}(r u h)+\frac{\partial}{\partial \sigma}(\omega h)=0,
$$

where $h$ defines "thickness" between isopycnals;

$$
h=-\frac{\partial z}{\partial \sigma} .
$$

Equation (4-8) relates temporal thickness change between isopycnals to radial divergence along the isopycnal and vertical divergence due to mixing, $\omega$.

The rate of change of potential temperature and salinity following a water parcel are governed by mixing as well;

$$
\begin{gathered}
\frac{\mathrm{d} \theta}{\mathrm{d} t}=H, \\
\frac{\mathrm{d} S}{\mathrm{~d} t}=E .
\end{gathered}
$$

Here, we define the temperature mixing and salinity mixing terms $H$ and $E$ respectively. $H$ and $E$ are related to $\omega$ in equation (4-7). Since density is a function of temperature and salinity, we can write,

$$
\frac{\mathrm{d} \sigma}{\mathrm{d} t}=\frac{\partial \sigma}{\partial \theta} \frac{\mathrm{d} \theta}{\mathrm{d} t}+\frac{\partial \sigma}{\partial S} \frac{\mathrm{d} S}{\mathrm{~d} t} .
$$

Defining the expansion coefficient for temperature and salinity as,

$$
\begin{aligned}
& \alpha=-\frac{\partial \sigma}{\partial \theta}, \\
& \beta=\frac{\partial \sigma}{\partial S},
\end{aligned}
$$


$\omega$ is linearly related to $H$ and $E$ as,

$$
\omega=-\alpha H+\beta E .
$$

The material derivative, $d / d t$, appearing in equation (4-10) is, in density coordinates,

$$
\frac{\mathrm{d}}{\mathrm{d} t}=\frac{\partial}{\partial t}+u \frac{\partial}{\partial r}+\omega \frac{\partial}{\partial \sigma} \text {. }
$$

The first term is the temporal change on an isopycnal while the other two are changes due to advection along an isopycnal, and across an isopycnal due to mixing.

The azimuthal velocity $(v)$ is in gradient wind balance (equation $4-1)$. The evolution equation of this azimuthal swirl is given by the azimuthal momentum equation (note $u \ll v$ ),

$$
\frac{\mathrm{d} v}{\mathrm{~d} t}+\frac{u v}{r}+f u=F,
$$

where $F$ is the azimuthal component of the frictional forcing. Multiplying equation (4-15) with $r$ and combining terms gives the angular momentum equation,

$$
\frac{\mathrm{d} M}{\mathrm{~d} t}=r F
$$

where angular momentum $M$ is defined by equation (4-3). Thus, in the absence of friction, angular momentum behaves as a conserved tracer just as potential temperature and salinity.

Potential vorticity $Q$, defined in equation (4-4), is a function of angular momentum and thickness.

$$
Q=\frac{f+1 / r\{\partial / \partial r(r v)\}}{\rho_{0} h}=\frac{1}{\rho_{0} r h} \frac{\partial M}{\partial r} .
$$

The governing equation for $Q$ is obtained by combining equations (4-8) and (4-16),

$$
\frac{\mathrm{d} Q}{\mathrm{~d} t}=\frac{1}{r h} \frac{\partial}{\partial r}(r F)+\frac{1}{r h}\left(\frac{\partial M}{\partial r} \frac{\partial \omega}{\partial \sigma}-\frac{\partial M}{\partial \sigma} \frac{\partial \omega}{\partial r}\right)
$$


Thus, information of the temporal change in potential vorticity is implicitly present in equations (4-8) and (4-16).

Equations (4-6), (4-8), (4-10), and (4-16) relate the temporal property changes on each isopycnal to the meridional circulation $(u, w)$ and diffusive activities $(\omega, H, E$, and $F$ ). Rewriting these equations putting the observed temporal changes on the right hand side and terms involving the unknown circulation and mixing on the left hand side we have,

$$
\begin{aligned}
\frac{1}{r} \frac{\partial}{\partial r}(r h u)+\frac{\partial}{\partial \sigma}(\omega h) & =-\frac{\partial h}{\partial t}, \\
u \frac{\partial \theta}{\partial r}+\omega \frac{\partial \theta}{\partial \sigma}-H & =-\frac{\partial \theta}{\partial t}, \\
u \frac{\partial S}{\partial r}+\omega \frac{\partial S}{\partial \sigma}-E & =-\frac{\partial S}{\partial t}, \\
u \frac{\partial M}{\partial r}+\omega \frac{\partial M}{\partial \sigma}-r F & =-\frac{\partial M}{\partial t} .
\end{aligned}
$$

The mixing terms can be expressed in terms of eddy diffusivities and eddy viscosities. Defining $\kappa_{i}$ and $\kappa_{d}$ to be isopycnal and diapycnal diffusivities respectively, $H$ can be written as,

$$
H=\frac{1}{r h} \frac{\partial}{\partial r}\left(\kappa_{i} r h \frac{\partial \theta}{\partial r}\right)+\frac{1}{h} \frac{\partial}{\partial \sigma}\left(\frac{\kappa_{d}}{h} \frac{\partial \theta}{\partial \sigma}\right) .
$$

$E$ is expressed similarly except $\theta$ replaced by $S$. Using the linear relation (4-13) and ignoring the nonlinearity of the equation of state (i.e. mixing along isopycnals does not change density, which is equivalent to say $\partial \alpha / \partial r=\partial \beta / \partial r=0), \omega$ can be expressed solely in terms of $\kappa_{d}$,

$$
\omega=\frac{1}{h}\left\{-\alpha \frac{\partial}{\partial \sigma}\left(\frac{\kappa_{d}}{h} \frac{\partial \theta}{\partial \sigma}\right)+\beta \frac{\partial}{\partial \sigma}\left(\frac{\kappa_{d}}{h} \frac{\partial S}{\partial \sigma}\right)\right\}
$$

The frictional term $F$ is written in terms of isopycnal and diapycnal eddy viscosities, $\nu_{i}$ and $\nu_{d}$ as,

$$
r F=\frac{1}{h}\left\{\nu_{i} r h \frac{\partial}{\partial r}\left(\frac{v}{r}\right)+\frac{\partial}{\partial r}\left(\nu_{i} r^{2} h \frac{\partial}{\partial r}\left(\frac{v}{r}\right)\right)\right\}+\frac{r}{h} \frac{\partial}{\partial \sigma}\left(\frac{\nu_{d}}{h} \frac{\partial v}{\partial \sigma}\right) .
$$




\begin{tabular}{|c|c|c|}
\hline grid & radial grid $(\mathrm{km})$ & depth grid $\left(\sigma_{\theta}\right)$ \\
\hline 1 & $0 .(1)$ & $26.77(1)$ \\
2 & $20 .(5)$ & $26.80(2)$ \\
3 & $40 .(9)$ & $26.83(3)$ \\
4 & $60 .(13)$ & $26.90(4)$ \\
5 & $75 .(16)$ & $27.10(6)$ \\
6 & $90 .(19)$ & $27.30(8)$ \\
7 & - & $27.47(10)$ \\
\hline
\end{tabular}

Table 4-2. Grid for Model 1

Numbers in parentheses are the radial grid numbers from ring center $(5 \mathrm{~km}$ interval) and isopycnal levels given in Table 4-1.

Thus equations (4-19) together with (4-20), (4-21), and (4-22) define a set of linear equations for the unknown parameters; $u, \kappa_{i}, \kappa_{d}, \nu_{i}$, and $\nu_{d}$. In matrix notation these equations are written,

$$
\mathbf{A} \underline{b}=-\underline{\Gamma}
$$

where $\mathbf{A}$ is a matrix, and $\underline{b}$ and $\underline{\Gamma}$ are column vectors. Each row of equation (423 ) is one of the equations in (4-19) at a certain isopycnal and radial position. The right hand side, $-\underline{\Gamma}$, is the temporal change of each tracer. $\underline{b}$ is the vector of unknown parameters. The coefficient matrix, $\mathbf{A}$, consists of spatial gradients of the tracers which appear in equations (4-19), (4-20), (4-21), and (4-22). Equation (4-23) defines a linear regression problem in which we invert the matrix equation to determine the unknown parameters $\underline{b}$. Inverse methods have received wide attention in oceanography in recent years. Wunsch (1978) gives a concise review of the mathematics involved in solving such linear inverse problems. A singular value decomposition will be used here in a series of models, in which the treatment of the mixing terms will vary.

In these models we use a subset of the mapping grid on which parameter estimates will be made. These are listed in Table 4-2, where we have 6 radial grid points and 7 isopycnal surfaces. (In addition to these, we use data at $r=100 \mathrm{~km}, \sigma_{\theta}=26.6$ 
and 27.58 to evaluate the spatial gradients on the grid points.) The entire extent of the grid is chosen with reference to ring translation and property distribution. Flierl (1981) shows in a simple kinematic model that the trapped region of a moving vortex in which water is advected with the translating eddy is delineated by the radius where the azimuthal velocity equals the translation speed of the vortex. According to Evans et al. (1985), ring $82 \mathrm{~B}$ translated at about $10 \mathrm{~cm} / \mathrm{sec}$ to the southwest between April and June. The azimuthal velocity in June decreases to this rate at about $90 \mathrm{~km}$ radius. In the vertical, azimuthal velocity becomes $10 \mathrm{~cm} / \mathrm{sec}$ at about $\sigma=27.65$. On the

other hand, the maximum depth where there is a radial contrast of potential vorticity between the ring's core and edge is limited to about $\sigma=27.53$. The radial and vertical intervals of this grid are chosen to resolve some of the different domains we see in the temporal changes (Section 2). The first 3 density surfaces are the top, middle, and bottom surfaces that define the thermostad of the ring. The top layer of the thermocline is between density levels 3 and 4 . This is the layer in which $Q$ decreased inside $75 \mathrm{~km}$, which is radial grid 5 . The near homogeneous $Q$ layer of the thermostad is inside radial grid 3.

\section{Model 1: Diffusivity-Viscosity}

In this first model, we estimate radial velocity and mixing coefficients at each separate grid point. Rather than specifying the mixing coefficients to be constant from the beginning, the model itself determines how the mixing coefficients should vary in space. In this model we use the equations for $h, \theta, S$, and $M$ on each of the 42 grid points in Table 4-2 (except for $M$ at ring center where it trivially remains 0 ) resulting in 161 equations. Among the parameters, $u$ is set to 0 at ring center. None of the viscosities at ring center appear in the constraints since $M$ as well as its spatial and temporal derivatives are 0 there. Thus we have 189 unknown parameters. 
The set of equations involves different dimensional quantities and thus must be nondimensionalized before they could be compared. This is accomplished by row weighting of the linear system (Wunsch, 1978). The row weights were calculated as the inverse of the row norm of the coefficient matrix $A$ for all models. Choice of this row weight is equivalent to asserting that we assume the relative accuracy of each constraint is proportional to its row norm. Additionally, each column is normalized (as well as the parameters) to keep different geometric factors from placing artificial emphasis on one parameter relative to another (Wunsch, 1978). Singular value decomposition was performed on this weighted system. The number of non-zero eigenvalues, or the rank of the problem, was determined as 110. (See for example Tziperman (1987a) or Rintoul (1988) for a discussion on rank determination.) The residual norm of equation (4-23) normalized by the norm of the right hand side is 0.16 .

The result of an inversion can only be interpreted within the context of the error and resolution of each parameter estimate. The standard error of the model parameter estimate is determined by the error in data on the right hand side of equation (4-23). These errors are calculated by using the residuals of equation (4-23) (Wiggins, 1972). Model parameter resolution indicates how well each parameter has been determined independently from another (Wunsch, 1978; Menke, 1984). A resolution of 1 would imply that the parameter is fully resolved, while a small resolution would mean the data do not contain enough information to determine the parameter.

The circulation of this solution is shown in Figure 4-14. The circulation is upward and radially out which reflects the result of the general relaxation of the thermocline. The radial velocities are on the order of several 10's of $\mathrm{m} /$ day. The resolutions of the $u$ 's are almost 1 everywhere in the thermocline, but are about 0.6 in the thermostad. The $u$ errors are almost uniform with values about $\pm 9 \mathrm{~m} /$ day. The vertical velocities in the thermocline are on the order of $1 \mathrm{~m} /$ day and are primarily due to the movement of the isopycnals. (The isopycnal movements are not model parameters. Therefore, the 


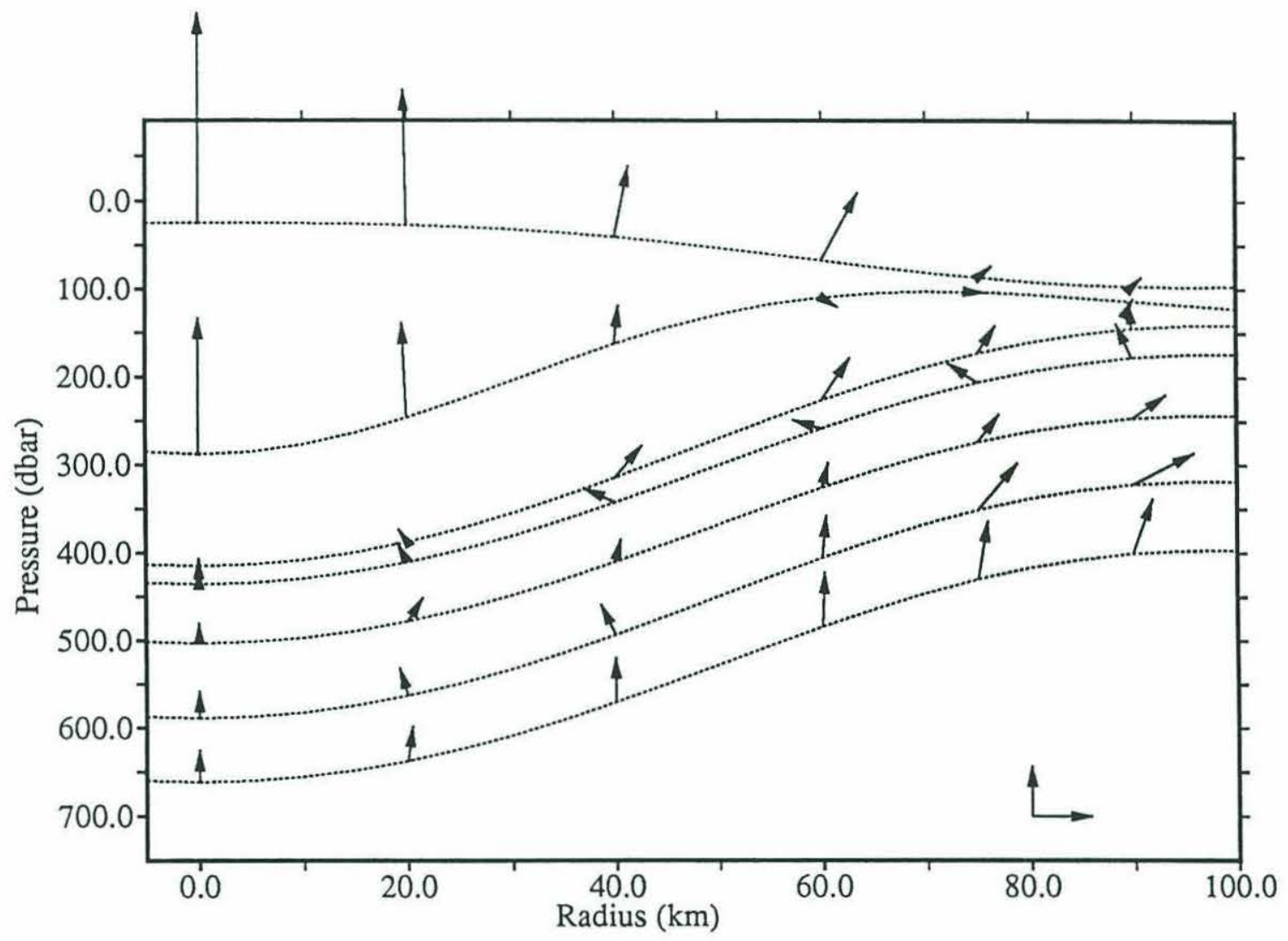

Figure 4-14. Circulation Estimate of Model 1

Velocities are drawn to scale. The reference vectors in the lower right hand corner are 100 (m/day) for $u$ and 1 (m/day) for $w$. 
errors involved are solely due to mapping, and are an order of magnitude smaller.) The vertical velocities are larger in the thermostad. Through the top of the thermostad, vertical velocity is dominated by cross isopycnal flow, which reflects the set up of the seasonal thermocline. However, as the errors of diapycnal diffusivities are large (described below), vertical velocity in the thermostad has an error as large as $30 \%$ of its value. There is a large vertical shear in radial velocity at the top of the thermocline. On the fourth isopycnal, the flow is radially inward in an otherwise outward circulation. The layer between the third and fourth isopycnals is where potential vorticity was seen to decrease with time. These two isopycnals are also where vertical shear of the azimuthal swirl is largest. Superficially, this reversal in radial flow is reminiscent of Csanady's (1979) model of the decay of a warm core ring by an interfacial Ekman layer, rather than Flierl and Mied's (1985) single celled stream function of a frictionally induced circulation. A reversal in radial flow was also estimated by Schmitz and Vastano (1976) for a decaying Gulf of Mexico anticyclonic ring. Examination of the inversion shows that the radial velocities at this depth are mostly constrained by the continuity equation.

Many of the mixing coefficients are not well resolved by this model. So, rather than discussing the parameters at each grid point, we shall examine their spatial mean in the thermostad and thermocline separately. The mean is taken inside $75 \mathrm{~km}$ (radial grid 5). These mean parameters are listed in Table 4-3 together with error estimates and mean resolutions. Among these means, diapycnal diffusivities are relatively well resolved. The errors of these means are estimated assuming that a parameter at each grid point is uncorrelated with another. This error is an optimistic estimate and could be larger by a factor of 5 . Judging conservatively, none of the mixing coefficients are truly distinguishable from zero.

Some of the eddy coefficients have negative means. Although negative mixing coefficients may be counterintuitive, there is no rigorous reason for them to be unacceptable 


\begin{tabular}{|c|c|c|}
\hline & Thermostad & Thermocline \\
\hline \multirow{3}{*}{$\kappa_{d}^{*}\left(\mathrm{~m}^{2} / \mathrm{sec}\right)$} & $0.34 \times 10^{-4}$ & $-0.98 \times 10^{-4}$ \\
& $0.11 \times 10^{-4}$ & $0.76 \times 10^{-5}$ \\
& 0.96 & 0.86 \\
\hline \multirow{3}{*}{$\kappa_{i}\left(\mathrm{~m}^{2} / \mathrm{sec}\right)$} & -5.97 & -1.50 \\
& 1.94 & 3.29 \\
& 0.77 & 0.32 \\
\hline & $0.79 \times 10^{-4}$ & $0.85 \times 10^{-4}$ \\
$\nu_{d}\left(\mathrm{~m}^{2} / \mathrm{sec}\right)$ & $0.19 \times 10^{-4}$ & $0.42 \times 10^{-4}$ \\
& 0.19 & 0.28 \\
\hline & 8.73 & -13.90 \\
$\nu_{i}\left(\mathrm{~m}^{2} / \mathrm{sec}\right)$ & 1.65 & 4.04 \\
& 0.41 & 0.67 \\
\hline
\end{tabular}

* Mid thermostad levels have been omitted from the average as they were not resolved compared to the ones above and below

Table 4-3. Mean Eddy Coefficients, Model 1

The first number is the mean, the second is the mean of the error, and the third is mean resolution of each parameter. 
in the present situation. The nonconservative terms in the constraint equations represent results of azimuthally averaging asymmetric structures of the ring as well as actual dissipation, which would include results of intermittent streamer activities (Evans et al., 1985). Later in another model we will determine if there is a consistent solution with positive mixing parameters.

Although many parameters are not well determined, it is interesting to compare the relative magnitude of each term appearing in the constraint equations, to examine the general character of what processes are dominant in each tracer's evolution. For example, in the thickness equation, diapycnal divergence caused by vertical mixing is seen to be the dominant term causing the reduced thickness in the thermostad. On the other hand, the controlling term in most parts of the thermocline is divergence due to radial advection. Diapycnal advection and diffusion are the governing processes in temperature and salinity changes. Isopycnal advection and diffusion are found somewhat important inside the thermostad about $r \sim 50 \mathrm{~km}$ where the radial temperature gradient is largest. Finally, angular momentum is controlled by isopycnal processes except at the top of the thermocline where diapycnal mixing also becomes significant.

\section{Other Models}

\subsection{Model 2: No Mixing}

Because of the low resolution of many of the mixing coefficients and their relatively large error estimates, one might anticipate that mixing need not be invoked to account for ring evolution. In this model we have set all mixing coefficients of model 1 to be zero a priori and have redone the inversion. The resulting circulation is shown in Figure 4-15. The overall circulation is similar to model 1 with comparable magnitudes. The

primary difference is the absence of cross isopycnal flow in this model at the top of the thermostad. However, the normalized residual norm of this model is 0.92 , and has 


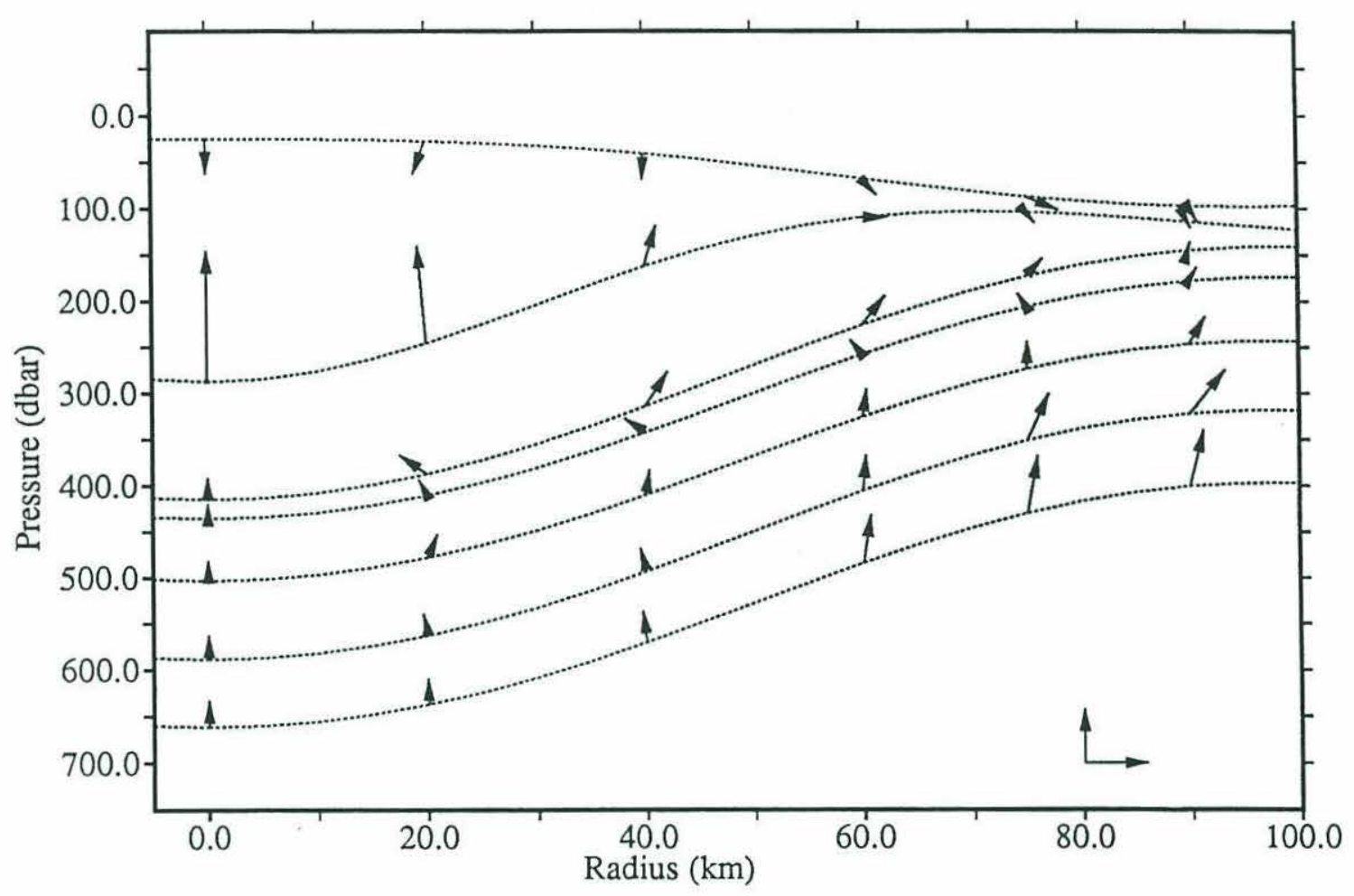

Figure 4-15. Circulation Estimate of Model 2

Velocities are drawn to scale as in Figure 4-14. 
hardly decreased from the null solution. Thus we conclude this model is inconsistent with the data and that mixing is essential in accounting for the ring's evolution.

\subsection{Model 3: Non-Parameterized Mixing}

Since mixing is essential but the individual parameters poorly resolved, we may want to primarily examine the circulation instead, while avoiding parameterizing the diffusive terms. The circulation is solely defined by $u$ and $\omega$, independent of the form of mixing parameterization. $\omega$ is a linear combination of temperature and salinity mixing terms, equation (4-13). Thus $u, H$, and $E$ fully determine the circulation without assuming any mixing parameterization. This formulation will also allow us to examine if temperature and salinity mixing could be determined separately, whereas their mixing parameters were the same in model 1 . In other words, this formalism could give us indications of whether double diffusive fluxes are important in the overall evolution of the ring. $u, H$, and $E$ are constrained by the $h, \theta$, and $S$ equations. The angular momentum equation may further constrain the parameters, but at a price of introducing an additional parameter, $F$. So to keep the problem from being unnecessarily large, we only use the $h, \theta$, and $S$ equations in this model.

For this model, we have used an alternative grid to that in model 1 , which is given in Table 4-4. (Data at $r=90 \mathrm{~km}$ and $\sigma_{\theta}=26.6$ and 27.62 are used to evaluate

spatial gradients.) The circulation estimate of this model is shown in Figure 4-16. The structure of the meridional circulation is again fairly similar to that of model 1 , despite the different formulation and grid structure. The circulation is upwards and radially out with a reversal of radial flow at the base of the thermostad. Although $u$ is well resolved, it turns out that $H$ and $E$ are not resolved independently, but only as a linear combination, $\omega$. Similarly, inspection of the inversion shows that the $\theta$ and $S$ equations are used only as a linear combination and they do not provide independent information. Thus we conclude that double diffusive fluxes are not resolvable with this 


\begin{tabular}{|c|c|c|}
\hline grid & radial grid (km) & depth grid (sigma) \\
\hline 1 & $0 .(1)$ & $26.77(1)$ \\
2 & $15 .(4)$ & $26.83(3)$ \\
3 & $30 .(7)$ & $27.00(5)$ \\
4 & $45 .(10)$ & $27.20(7)$ \\
5 & $60 .(13)$ & $27.40(9)$ \\
6 & $75 .(16)$ & $27.53(11)$ \\
\hline
\end{tabular}

Table 4-4. Model 3 Grid

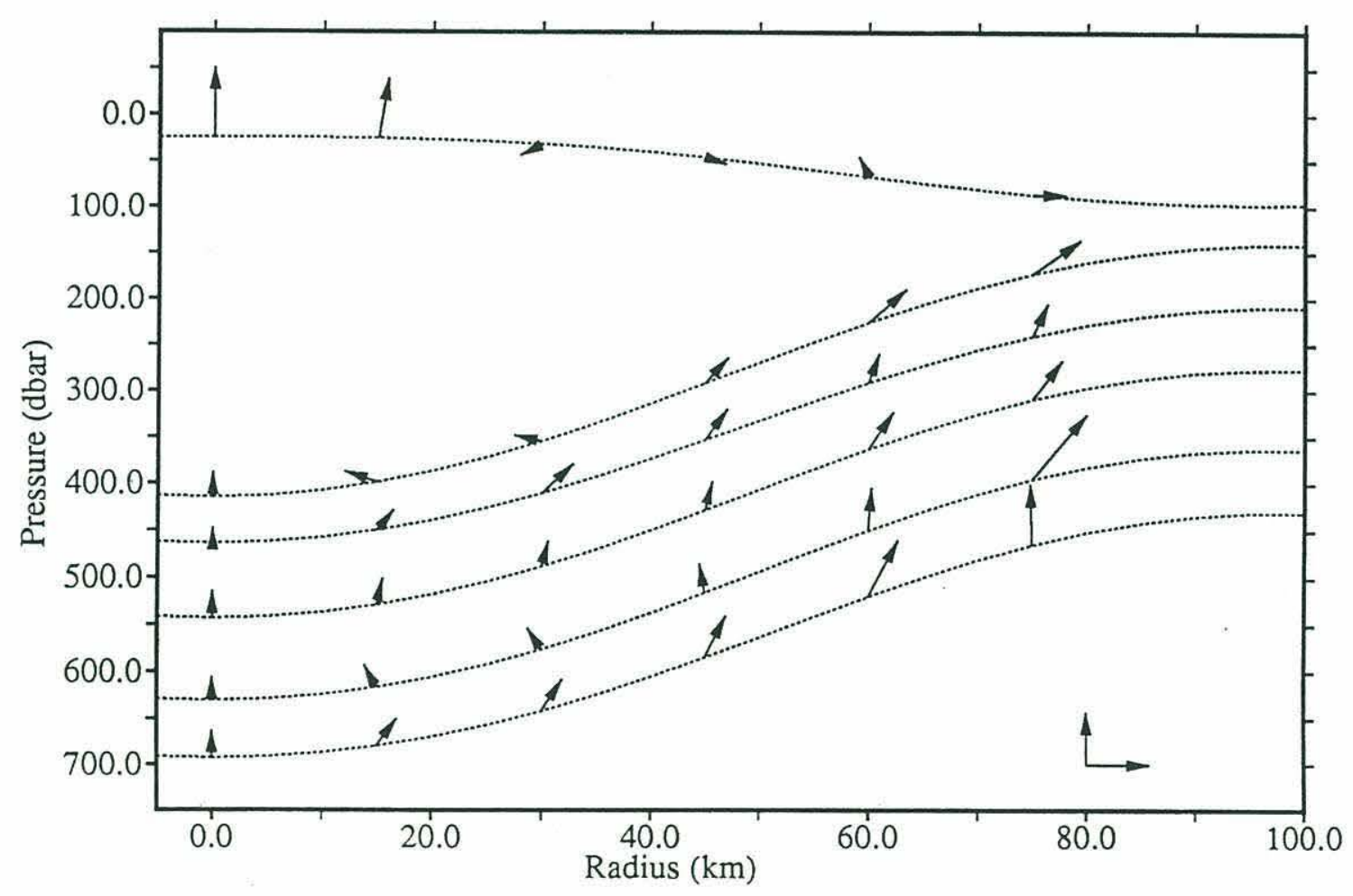

Figure 4-16. Circulation Estimate of Model 3

Velocities are drawn to scale as in Figure 4-14. 
data set and that treatment of the mixing coefficients to be tracer independent, as in model 1 , is both reasonable and gives consistent results.

\subsection{Model 4: Positive Mixing Coefficients}

The sign of the mixing parameters were not constrained in model 1. However, in a large scale sense, we would expect the dissipative fluxes to act down gradient to reduce the spatial gradients. In model 1 , it was anticipated that the model itself would determine the sign of the mixing parameters. Constraining the eddy coefficients to be non-negative might add information to the data to determine the solution better. In this model, we examine if there is a consistent solution while constraining the eddy coefficients to be positive. Mathematically, the problem is to minimize the residual of equation (4-23) under the additional linear inequality constraints,

$$
\kappa, \nu \geq 0
$$

which can be done with an extension of the singular value decomposition solution (Tziperman, 1987a).

When all the mixing coefficients are constrained to be positive, some of the $\theta$ and $S$ equations have large residuals. Most of the mixing coefficients that were negative in model 1 are set to their lower limit (0.). However, one of the eddy viscosities become exceedingly large (order $100 \mathrm{~m}^{2} / \mathrm{sec}$ ), which we conclude to be unrealistic.

One of the better resolved mixing parameters which was negative in the first model is the diapycnal diffusivity. In a model where we relax the positiveness condition on $\kappa_{d}$, the residual of the linear constraints become comparable with model 1 . The mixing parameters remain the same order of magnitude as well unlike the situation above. The circulation estimate, shown in Figure 4-17, is again extremely similar to that of the first model. 


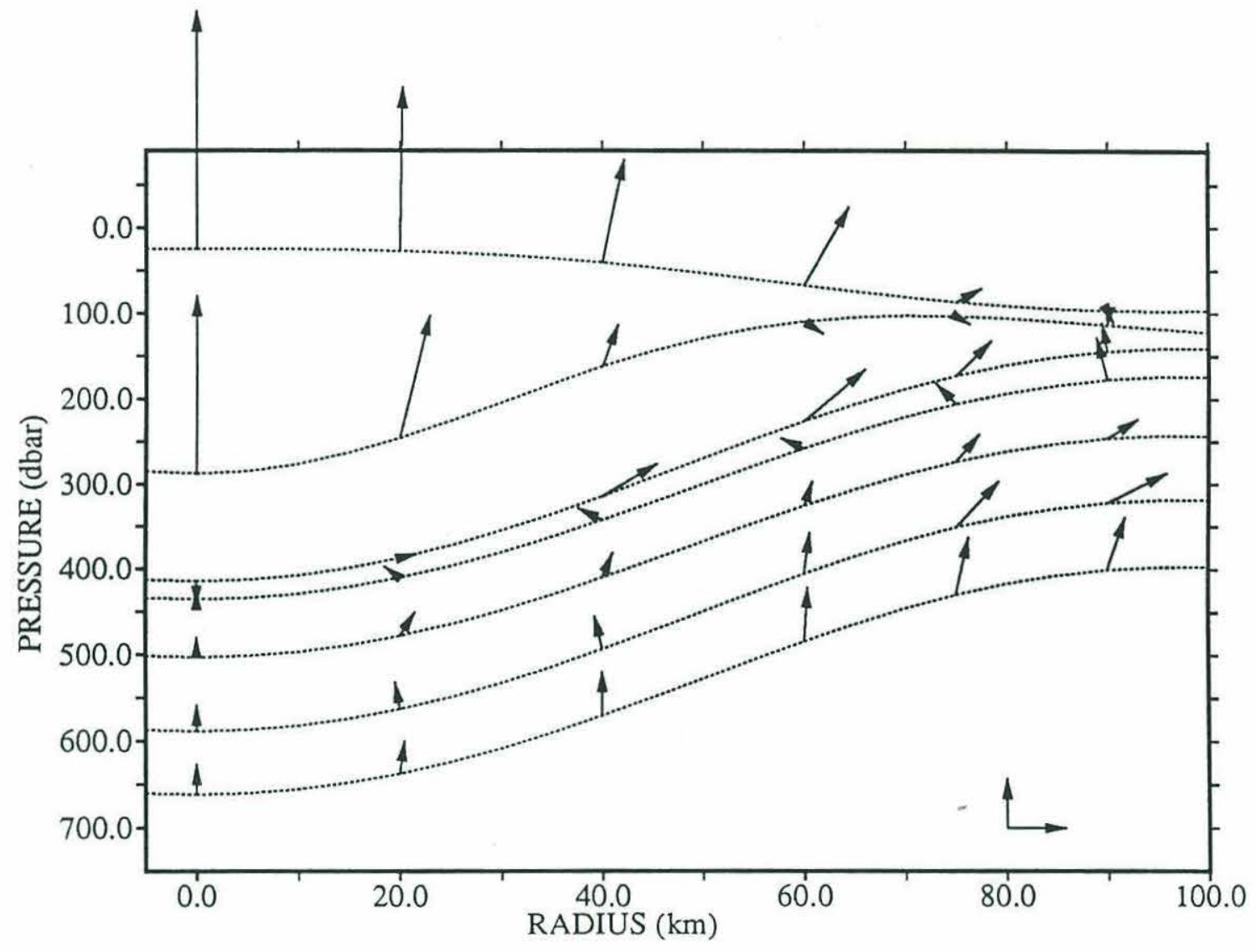

Figure 4-17. Circulation Estimate of Model 4

Velocities are drawn to scale as in Figure 4-14. 
A negative diffusivity means that the nonadiabatic flux is negatively correlated with the mean property gradient. Mixing terms in equation (4-19) are sums of various processes affecting the azimuthally averaged ring; Gulf Stream streamers and shelf streamers, ring-topography interaction, dispersion of Rossby waves, asymmetric perturbations including "ringlets" (Kennelly et al., 1985), etc. It is not altogether obvious that these processes when azimuthally averaged should be positively correlated with the mean structure of the ring. The negative diapycnal diffusivity seems to indicate otherwise.

\subsection{Model 5: Thermocline Only}

In the models we have analyzed so far, mixing is poorly resolved. This could be a result of some non-adiabatic processes that are not appropriately described by mixing coefficients. For example, intermittent streamers and jets are observed (e.g. Joyce and Stalcup, 1984) that can transport ambient water directly into ring center. Active convection is also observed in the thermostad (Schmitt and Olson, 1985) before the seasonal thermocline sets up. These processes may in effect act as internal sources of different water masses rather than characterizable by Fickian diffusion between ring and its surroundings. In hope of better determining the mixing parameters, we reanalyze Model 1 , by excluding the thermostad isopycnals where the above processes may be important. We will call this Model 5.

The average eddy coefficients (Table 4-5), which are calculated as in Model 1, are indistinguishable from the previous results (Table 4-3). (The circulation estimate, not shown here, is also consistent with the result of Model 1.) Most mixing coefficients are still poorly resolved and the diapycnal diffusivities still have a negative mean value. Thus the poor resolution of the Model 1 mixing coefficients is not a result of the inclusion of the thermostad in the model, but is a result of the indeterminancy of the thermocline data themselves. 


\begin{tabular}{|c|c|c|}
\hline & Model 5 & Model 5-b \\
\hline \multirow{3}{*}{$\kappa_{d}\left(\mathrm{~m}^{2} / \mathrm{sec}\right)$} & $-1.04 \times 10^{-4}$ & $-0.53 \times 10^{-4}$ \\
& $0.17 \times 10^{-4}$ & $0.10 \times 10^{-4}$ \\
& 0.86 & 1.00 \\
\hline & -1.12 & -16.54 \\
$\kappa_{i}\left(\mathrm{~m}^{2} / \mathrm{sec}\right)$ & 5.63 & 3.97 \\
& 0.32 & 0.96 \\
\hline & $0.74 \times 10^{-4}$ & $-0.03 \times 10^{-4}$ \\
$\nu_{d}\left(\mathrm{~m}^{2} / \mathrm{sec}\right)$ & $0.72 \times 10^{-4}$ & $0.30 \times 10^{-4}$ \\
& 0.25 & 0.57 \\
\hline & -13.90 & -6.57 \\
$\nu_{i}\left(\mathrm{~m}^{2} / \mathrm{sec}\right)$ & 7.19 & 2.90 \\
& 0.65 & 0.32 \\
\hline
\end{tabular}

Table 4-5. Model 5; Mean Thermocline Eddy Coefficients

As in Table 4-3, the first number is the mean, the second is the mean of the error, and the third is mean resolution of each parameter. 
Finally, we examine a variation of this Model 5, where we further constrain each mixing coefficient to be spatially uniform (Model 5-b). By adding this additional information about spatial variation to Model 5, we may hope to determine the mean coefficients better. The resulting mixing coefficients for Model 5-b are also given in Table 4-5. The viscosities are still poorly resolved but isopycnal diffusion appears to be better determined. Diapycnal diffusivity has slightly smaller magnitude, but is not inconsistent with the Model 5 result. However, some of the constraint equations have large residuals for this model. The normalized residual norm for this model is 0.76 , whereas this is 0.14 in Model 5. This large residual norm indicates that this model is underparameterized and uniform mixing coefficients do not explain different temporal changes observed in the ring simultaneously.

\section{Summary}

The spatial and temporal changes of warm core ring 82B's properties imply mixing is an important factor in ring decay. A series of models was constructed to determine the circulation and mixing that underlie the observed temporal evolution of the ring's structure. The circulation estimate is well resolved and robust, in the sense that all models result in a very similar pattern. The basic circulation is a general upward and radially outward flow except for an inflow beneath the thermostad. Radial velocities are of several 10's of m/day and vertical velocities are on the order of $1 \mathrm{~m} /$ day. The vertical velocity is mostly accounted for by the vertical movement of the isopycnals, except at the top of the thermostad where there is significant diapycnal velocity.

On the other hand, the resolution of independent mixing parameters by the data are poor and the error is large. However, this low resolution does not mean that mixing is insignificant. An adiabatic model was found inconsistent with the data. Some of the estimated eddy mixing coefficients are negative, but again the error and resolution is such that they are not statistically distinguishable from zero. In fact it 
is possible to find a solution with most of the mixing parameters being non-negative. The data are inconclusive in determination of individual mixing parameter estimates. In one of the models where we avoid parameterizing mixing, it turns out what we could only resolve is the rate of density change, $\omega$. Temperature and salinity do not provide independent information, so that differences in their mixing do not seem to be significant in accounting for the ring's evolution, at least to the extent the data can resolve them.

In conclusion, the inversion has shown that changes in ring structure can be explained consistently by a simple diagnostic model. However, the mixing parameters are poorly determined by the data. The simple advection-diffusion model is too complex compared to what the data can resolve. What we need is a more accurate data of the ring evolution. The shape of the ring is not exactly a circle but more elliptical. A circular average will sum over different elliptical streamlines and reduce the radial resolution of the ring. This could cause artificial structural changes that mimics the effect of radial diffusion. Temporal resolution is important as well, as there are intermittent streamers and jets that penetrate into the ring (Joyce and Stalcup, 1984). These streamers can transport different water mass and momentum directly inside the ring, unlike the gradual penetration of diffusion. Inclusion of such phenomena would make the model more complicated and demanding than the present data warrant. 


\section{Concluding Remarks}

In the preceding four chapters we have examined the modal structure of the North Atlantic Ocean hydrography, and have applied inverse methods to two separate ocean circulation problems. We now briefly review the results of the analyses and consider where the results lead to.

The first two chapters demonstrated that the ocean hydrographic structure can be adequately described by a small number of modes (empirical orthogonal functions). The structures of the modal expansion coefficients reflect known water mass distributions, thus reflecting the underlying physics. There are some limited places with large residuals, but these places in turn also indicate the presence of different physics; e.g. deep western boundary currents.

The low mode representation of the hydrography is an efficient description, which makes data handling easier. Simplicity, however, does not immediately mean we can do away with the various observations. We still need the full depth profiles of properties to obtain the modes, since we cannot observe the modes directly. On the other hand, the correlation among different tracers do imply that we may not need every tracer to study ocean circulation, at least to the degree of complexity the low modes resolve.

What the modes resolve is an important question. One of the main goals in oceanography is to understand global circulation. The World Ocean Circulation Ex-

periment (WOCE International Planning Office, 1988) has set goals along such a line by planning intensive observations of the global ocean. However, it is becoming more evident that syntheses of various observations are going to be difficult in light of the sampling requirements to resolve the transient eddies. We probably will not be able to describe the ocean synoptically in every detail using hydrographic data. The modes, by identifying the most significant and essential hydrographic structure of the ocean, provide an alternative objective framework from which one can construct an ocean model 
of various complexities. In a sense, the modes are akin to scaling of the hydrographic structure. The small number of modes essentially provide a basis for a first order ocean model. By examining the physics of such first order models, we may hope to elucidate global ocean physics which otherwise may be too complicated to analyze.

An attempt to construct such a first order ocean model was the mapped result presented in Section 8.4 of Chapter 2. The circulation determined over a $12^{\circ}$ square of this model ocean demonstrated that such an ocean is consistent to conventional wisdom about the region's physics. There is no apparent loss of physics in such modeling.

The ocean model in Chapter 2 was constructed by objective mapping. Such a simple interpolation/extrapolation is acceptable as a first step. But, it is obvious that the information necessary to interpolate modal coefficients must come from other observations or dynamical constraints. Satellite altimetry, for its ability to sample the ocean, is promising to measure some of the important statistics. Theoretical ocean circulation models will provide the essential dynamical constraints. The challenge then is to express these and other data and models in terms of the modes, which will make the assimilation of such information with the hydrographic information straightforward. The problem is to find ways to relate modes to other physics (models and observations) without recreating the physical space; i.e. temperature and other properties as a function of depth. This is something one needs to explore in the future.

The modes revealed that surface topography, and thus surface circulation, is well reproduced by an even smaller set of modes: two modes as opposed to the six modes necessary to account for the global structure. This good agreement of surface dynamic height indicates that knowledge of surface topography such as from satellite altimetry can provide strong constraints on what the spatial variation of the gravest modes are. On the other hand, altimetry will not likely contain much information about the higher modes. In contrast with sea surface height, sea surface temperature is not likely 
to be a good predictor of subsurface structure, as suggested by the large sea surface temperature residuals.

Chapters 3 and 4 were applications of inverse methods to two separate problems in ocean circulation. In Chapter 3, circulation over the Mediterranean Water tongue in the eastern North Atlantic Ocean was examined based on the modal model of Chapter 2. Chapter 4 examined the meridional circulation and mixing associated with the decay process of a warm-core ring. The circulation example in Chapter 3 was a geostrophic flow, while the flow in Chapter 4 was an ageostrophic circulation.

Both inverse method applications resolve the circulation well. The circulation in Chapter 3 consists of an anticyclonic circulation near the surface and a weak (order $\mathrm{mm} / \mathrm{sec}$ ) cyclonic flow at depth circulating around the core of the Mediterranean Water tongue. The meridional circulation of the warm-core ring is upwelling and radial outflow.

On the other hand, mixing was not well resolved in either model. Eddy coefficients were only partially resolved in Chapter 4 . The $12^{\circ}$ region's density structure was explained consistently without invoking mixing: i.e. we cannot resolve mixing processes. The situation in Chapter 4 is where we are following an isolated water mass; i.e. the ring. Even in this relatively simple situation, mixing is a difficult quantity to resolve. These two examples suggest that much more accurate data is necessary to determine the eddy coefficients in the ocean with any degree of confidence.

Although the inversion done in Chapter 3 was based on the modal model, the actual inversion was performed as with traditional data; i.e. data on a set of depths. The mapping of the data and the set-up of the inverse problem were economized, but the actual inversion does not capitalize on the underlying simplicity of the hydrographic structure. The equations that are solved simultaneously are similar; an equation at one station is almost the same as the equation at a neighboring grid point. The vertical discretization (equations for layers or for depth levels) is not optimal. Ideally one would 
like to determine circulation directly from the modal coefficients. The question is to reduce the mathematics of the inversion; the matrix equation involved in the inversion contains many similar equations (rows). This is yet another problem one would need to examine in the future.

A comprehensive understanding of the ocean physics will ultimately be achieved by a quantitative syntheses of various observations and theoretical models. The issue of syntheses is an important one, especially in light of the upcoming World Ocean Circulation Experiment, where observations of unprecedented scope along with community efforts in generating the next generation circulation models are being planned. Assimilation of the raw data with models is unlikely, considering the enormous volume of the data that must be handled. We must therefore seek ways to reduce the numerical dimensionality to something more feasible. The preceding analyses of this thesis have examined how to reduce the hydrographic structure of the ocean. Although the results are not definitive (we do not claim that our modes are the correct ones) and there remains some issues to be examined in the future, the problem of data reduction is an important matter and the work presented here is in the right direction. The framework devised here is a first step towards constructing a comprehensive model of the ocean. 


\section{Acknowledgments}

I would first like to thank my thesis advisor, Carl Wunsch, for the invaluable guidance and support he provided me throughout the course of this research. I have benefited greatly from Carl's enlightening advice and exposure to his own work. They were crucial in shaping the direction of my interests and helpful in keeping the work going and putting the problems and their results into perspective. I am fortunate to have been Carl's student.

I am also grateful to the other members of my thesis committee - Glenn Flierl, Terry Joyce, and Mike McCartney — for giving me helpful comments and suggestions, and for generously sparing their time for me. Terry also gave me access to the warmcore ring data, and Mike provided many of the hydrographic section data.

Thanks are also due to Barbara Grant and Charmaine King for their able assistance in all computing matters. Ross Hendry kindly provided the access to the $48^{\circ} \mathrm{N}$ data before publication. Most of the figures were produced by a plotting macro package developed by Reiner Schlitzer. David Rothstein helped contour many of the section data. Many other friends, fellow students and post-docs, have contributed along the way to help me get through and put up with me whenever I " didn't understand " what the computer was spitting back.

My special thanks go to my wife, Haruko, who is my moral support and advisorat-large. Her love has made even the intolerable endurable and was essential in my completion of this work.

Finally, I want to express my deepest gratitude to my parents for their unfailing support through all these years. I would like to dedicate this thesis to them.

This research was funded in part by the Office of Naval Research (Secretary of the Navy Chair) and the National Science Foundation under grant OCE 85-21685. 


\section{References}

Arhan, M., 1987. On the large scale dynamics of the Mediterranean outflow. Deep-Sea Res., 34, 1187-1208.

Armi, L., and H. Stommel, 1983. Four views of a portion of the North Atlantic subtropical gyre. J. Phys. Oceanogr., 13, 828-857.

Behringer, D. W., 1979. On computing the absolute geostrophic velocity spiral. J. Mar. Res., 37, 459-470.

Behringer, D. W., and H. Stommel, 1980. The beta spiral in the North Atlantic subtropical gyre. Deep-Sea Res., 27A, 225-238.

Bracewell, R. N., 1978. The Fourier Transform and its Applications, McGraw Hill, New York, 444 pp.

Bretherton, F. P., R. E. Davis, and C. B. Fandry, 1976. A technique for objective analysis and design of oceanographic experiments applied to MODE-73. Deep-Sea Res., 23, 559-582.

Brewer, P. G., J. L. Sarmiento, and W. M. Smethie, Jr., 1985. The Transient Tracers in the Ocean (TTO) Program: The North Atlantic study, 1981; The Tropical Atlantic study, 1983. J. Geophys. Res., 90, 6903-6905.

Broecker, W. S., and T. H. Peng, 1982. Tracers in the Sea. Lamont-Doherty Geological Observatory, Columbia University, Palisades, New York, 690 pp.

Brown, O. B., P. C. Cornillon, S. R. Emmerson, and H. M. Carle, 1986. Gulf Stream warm rings: a statistical study of their behavior, Deep Sea Res., 39, 1459-1473.

Cheney, R, and P. Richardson, 1976. Observed decay of a cyclonic Gulf Stream ring, Deep Sea Res., 2S, 143-156.

Csanady, G. T., 1979. The birth and death of a warm core ring, J. Geophys. Res., 84, $777-780$.

Davis, R. E., 1976. Predictability of sea surface temperature and sea level pressure anomalies over the North Pacific Ocean. J. Phys. Oceanogr., 6, 249-266.

Evans, R. H., K. S. Baker, O. B. Brown, and R. C. Smith, 1985. Chronology of warmcore ring 82B. J. Geophys. Res., 90, 8803-8811.

Flierl, G. R., 1981. Particle motions in large amplitude fields, Geophys. Astrophys. Fluid Dyn., 18, 39-74.

Flierl, G. R., and R. P. Mied, 1985. Frictionally induced circulations and spin down of a warm-core ring, J. Geophys. Res., 90, 8917-8927.

Fofonoff, N. P., 1981. The Gulf Stream System, in Evolution of Physical Oceanography, edited by B. Warren and C. Wunsch, MIT Press, Cambridge, Mass., 112-139. 
Fu, L.-L., J. Vazquez, and M. E. Parke, 1987. Seasonal variability of the Gulf Stream from satellite altimetry. J. Geophys. Res., 92, 749-754.

Hogg, N. G., 1987. A least-squares fit of the advective-diffusive equations to Levitus Atlas data. J. Mar. Res., 45, 347-375.

Hooker, S. B., and D. B. Olson, 1984. Center of mass estimation in closed vortices: A verification in principle and practice, J. Atmos. Oceanic Technol., 1, 247-255.

Jollife, I. T., 1986. Principal Component Analysis, Springer Verlag, New York.

Joyce, T. M., 1984. Velocity and hydrographic structure of a Gulf Stream warm core ring, J. Phys. Oceanogr., 14, 936-947.

Joyce, T. M., et al., 1984. Rapid evolution of a Gulf Stream warm-core ring, Nature, \$08, 837-840.

Joyce, T. M., and M. C. Stalcup, 1984. An upper-ocean current jet and internal waves in a Gulf Stream warm-core ring, J. Geophys. Res., 89(C2), 1997-2003.

Joyce, T. M., and M. A. Kennelly, 1985. Upper-ocean velocity structure of Gulf Stream warm-core ring 82B. J. Geophys. Res., 90, 8839-8844.

Kennelly, M. A., R. H. Evans, and T. M. Joyce, 1985. Small-scale cyclones on the periphery of a Gulf Stream warm-core ring, J. Geophys. Res., 90, 8845-8857.

Knapp, G. P., H. M. Stommel, 1985. Hydrographic data from R.V. Oceanus cruise 133, leg VII. Woods Hole Oceanographic Institution, Technical Report, No.85-38, 107 pp.

Knapp, G. P., 1988. Hydrographic data from R.V. Endeavor cruise 129. Woods Hole Oceanographic Institution, Technical Report, No.88-41, $111 \mathrm{pp}$.

Lawson, C. L., and R. J. Hanson, 1974. Solving Least Squares Problems, Prentice-Hall, Englewood Cliffs, N.J., 340 pp.

Levitus, S., 1982. Climatological atlas of the world ocean, NOAA Tech. Pap., 3, 173 pp.

Lorenz, E. N., 1956. Empirical Orthogonal Functions and Statistical Weather Prediction. Sci. Rep., No. 1, Statistical Forecasting Project-Massachusetts Institute of Technology.

McWilliams, J. C., and G. R. Flierl, 1979. On the evolution of isolated nonlinear vortices, J. Phys. Oceanogr., 9, 1159-1182.

Menke, W., 1984. Geophysical Data Analysis: Discrete Inverse Theory, Academic Press, Orlando, Florida, 260 pp.

Mied, R., and G. Lindemann, 1979. The propagation and evolution of cyclonic Gulf Stream rings, J. Phys. Oceanogr., 9, 1183-1206.

Montgomery, R. B., 1938. Circulations in upper layers of southern North Atlantic deduced with use of isentropic analysis. Papers in Physical Oceanography and Meteorology, 6:2, 55 pp. 
Needler, G. T., 1985. The absolute velocity as a function of conserved measurable quantities. Prog. Oceanogr., 14, 421-429.

Olbers, D. J., M. Wenzel and J. Willebrand, 1985. The inference of North Atlantic circulation patterns from climatological hydrographic data. Rev. Geophys., 2s, 313-356.

Olson, D. B., R. W. Schmitt, M. Kennelly, and T. M. Joyce, 1985. A two-layer diagnostic model of the long-term physical evolution of warm-core ring $82 \mathrm{~B}, J$. Geophys. Res., 90, 8813-8822.

PACODF, 1986a. TTO North Atlantic Study. Shipboard physical and chemical data report. Scripps Institution of Oceanography, San Diego, SIO reference No. 86-15.

PACODF, 1986b. TTO Tropical Atlantic Study. Shipboard physical and chemical data report. Scripps Institution of Oceanography, San Diego, SIO reference No. 86-16.

Price, J. F., T. K. McKee, J. R. Valdes, P. L. Richardson, and L. Armi, 1986. SOFAR float Mediterranean outflow experiment data from the first year, 1984-1985. Woods Hole Oceanographic Institution, Technical Report, No.86-31, 199 pp.

Provost, C., and R. Salmon, 1986. A variational method for inverting hydrographic data. J. Mar. Res., 44, 1-34.

Reid, J. L., 1978. On the middepth circulation and salinity field in the North Atlantic Ocean. J. Geophys. Res., 8s, 5063-5067.

Richardson, P. L., and K. Mooney, 1975. The Mediterranean outflow - A simple advection-diffusion model. J. Phys. Oceanogr., 5(3), 476-482.

Rintoul, S. R., 1988. Mass, heat and nutrient fluxes in the Atlantic Ocean determined by inverse methods. Ph.D. thesis, MIT/WHOI, Joint Program Phys. Oceanogr., Cambridge, Mass. 287 pp.

Roemmich, D., 1980. The application of inverse methods to problems in ocean circulation. Ph.D. thesis, MIT/WHOI, Joint Program Phys. Oceanogr., Cambridge, Mass. 193 pp.

Roemmich, D. and C. Wunsch, 1985. Two transatlantic sections: meridional circulation and heat flux in the subtropical North Atlantic Ocean. Deep-Sea Res., 32, 619-664.

Schmitt, R. W. and D. B. Olson, 1985. Wintertime convection in warm-core rings: Thermocline ventilation and the formation of mesoscale lenses, J. Geophys. Res., $90,8823-8837$.

Schmitz, J., and A. Vastano, 1975. Entrainment and diffusion in a Gulf Stream cyclonic ring, J. Phys. Oceanogr., 5, 93-97.

Schmitz, J., and A. Vastano, 1976. On entrainment and diffusion in a Gulf of Mexico anticyclonic ring, J. Phys. Oceanogr., 6, 399-402. 
Schmitz, J., and A. Vastano, 1977. Decay of a shoaling Gulf Stream cyclonic ring, $J$. Phys. Oceanogr., 7, 479-483.

Schmitz, W. J., Jr., J. F. Price, and P. L. Richardson, 1988. Recent moored current meter and SOFAR float observations in the eastern Atlantic near 32N. J. Mar. Res., 46, 301-319.

Schott, F., and H. Stommel, 1978. Beta-spirals and absolute velocities in different oceans. Deep-Sea Res., 25, 961-1010.

Schott, F., and R. Zantopp, 1979. Calculation of absolute velocities from different parameters in the western North Atlantic. J. Geophys. Res., 84, 6990-6994.

Schroeder, E. H., H. Stommel, D. Menzel, W. Sutcliff, Jr., 1959. Climatic stability of eighteen degree water at Bermuda. J. Geophys. Res., 64, 363-366.

Smith, R. C., and K. S. Baker, 1985. Spatial and temporal patterns in pigment biomass in Gulf Stream warm-core ring 82B and its environs, J. Geophys. Res., 90, 88598870 .

Stommel, H., and F. Schott, 1977. The beta spiral and the determination of the absolute velocity field from hydrographic station data, Deep-Sea Res., 24, 325-329.

Suk, M.-S., 1985. Sur la circulation saisonnière moyenne dans l'Atlantique Tropical en utilisant une méthode variationnelle, Thèse de Troisième Cycle at University of Paris VI, Paris, France. (in English).

Sverdrup, H. U., M. W. Johnson, and R. H. Fleming, 1942. The Oceans - Their Physics, Chemistry, and General Biology, Prentice-Hall, Englewood Cliffs, N.J., $1087 \mathrm{pp}$.

Tomosada, A, 1986. Generation and decay of Kuroshio warm-core rings, Deep Sea Res., $39,1475-1486$.

TOPOGULF group, 1986. Data report - volume 1: CTD, $\mathrm{O}_{2}$, and Nutrients. Berichte aus dem Institut für Meereskunde, Kiel, Germany, No. 154.

Tziperman, E., 1987a. Mixing and general circulation dynamics: Theory and observations, Ph.D. thesis, MIT-WHOI Joint Program Phys. Oceanogr., Cambridge, Mass. $162 \mathrm{pp}$.

Tziperman, E., 1987b. The Mediterranean outflow as an example of deep buoyancydriven flow. J. Geophys. Res., 92, 14,510-14,520.

Wallace, J. M., and R. E. Dickinson, 1972. Empirical orthogonal representation of time series in the frequency domain. Part I: theoretical considerations. J. Appl. Meteor., $11,887-892$.

Wallace, J. M., and D. S. Gutzler, 1981. Teleconnections in the geopotential height field during the northern hemisphere winter. Mon. Wea. Rev., 109, 784-812. 
Warm Core Rings Executive Committee, 1982. Multidisciplinary program to study warm-core rings, Eos Trans. AGU, 63, 834-836.

Wiggins, R. A., 1972. The general linear inverse problem: Implication of surface waves and free oscillations for earth structure. Rev. Geophys. Space Phys., 10, 251-285.

WOCE International Planning Office, 1988. World Ocean Circulation Experiment Implementation Plan. World Meteorological Organization, WMO/TD No.242.

Worthington, L. V., and W. G. Metcalf, 1961. The relationship between potential temperature and salinity in deep Atlantic water. Rapports et Procès-Verbaux des Réunions, Conseil Permanent International pur l'Exploration de la Mer, 149, 122128.

Worthington, L. V., 1976. On the North Atlantic Circulation, in Johns Hopkins Oceanogr. Stud., Johns Hopkins University, Baltimore, Md., vol. 6, 110 pp.

Worthington, L. V., 1981. The water masses of the world ocean: some results of a fine-scale census, in Evolution of Physical Oceanography, edited by B. Warren and C. Wunsch, MIT Press, Cambridge, Mass., 42-69.

Wright, W. R., and L. V. Worthington, 1970. The Water Masses of the North Atlantic Ocean; a Volumetric Census of Temperature and Salinity. Serial Atlas of the Marine Environment, Folio 19, American Geographical Society, New York, 8 pp. and 7 plates.

Wunsch, C., 1977. Determining the general circulation of the oceans: A preliminary discussion, Science, 196, 871-875.

Wunsch, C., 1978. The general circulation of the North Atlantic west of $50^{\circ} \mathrm{W}$ determined from inverse methods. Reviews of Geophysics and Space Physics, 16, 583-620.

Wunsch, C., and B. Grant, 1982. Towards the general circulation of the North Atlantic Ocean. Prog. Oceanogr., 11, 1-59.

Wüst, G.,1935. Schichtung und Zirkulation des Atlantischen Ozeans. Die Stratosphäre. In Wissenschaftliche Ergebnisse der Deutschen Atlantischen Expedition auf dem Forschungs-und Vermessungsschiff "Meteor" 1925-1927, 6: 1st Part, 2, 180 pp. (The Stratosphere of the Atlantic Ocean, W.J.Emery, ed., 1978, Amerind, New Delhi, 112 pp.)

Zenk, W., and T. J. Müller, 1988. Seven-year current meter record in the eastern North Atlantic. Deep-Sea Res., 8, 1259-1268. 